\title{
Development of Carbon Fiber Reinforced Stellite Alloy Based Composites for Tribocorrosion Applications
}

by

\begin{abstract}
Alireza Khoddamzadeh
B. Eng., M.A.Sc. Materials
\end{abstract}

A thesis submitted to the Faculty of Graduate and Postdoctoral Affairs in partial fulfilment of the requirements

for the degree of

Doctor of Philosophy

in

\section{Mechanical Engineering}

\author{
Ottawa-Carleton Institute for \\ Mechanical and Aerospace Engineering \\ Department of Mechanical and Aerospace Engineering \\ Carleton University \\ Ottawa, Ontario, Canada \\ June, 2012
}

Copyright $@ 2012$

Alireza Khoddamzadeh 
Library and Archives

Canada

Published Heritage

Branch

395 Wellington Street

Ottawa ON K1A ON4

Canada
Bibliothèque et

Archives Canada

Direction du

Patrimoine de l'édition

395 , rue Wellington

Ottawa ON K1A ON4

Canada
Your file Votre référence

ISBN: 978-0-494-93654-2

Our file Notre référence

ISBN: $978-0-494-93654-2$
NOTICE:

The author has granted a nonexclusive license allowing Library and Archives Canada to reproduce, publish, archive, preserve, conserve, communicate to the public by telecommunication or on the Internet, loan, distrbute and sell theses worldwide, for commercial or noncommercial purposes, in microform, paper, electronic and/or any other formats.

The author retains copyright ownership and moral rights in this thesis. Neither the thesis nor substantial extracts from it may be printed or otherwise reproduced without the author's permission.
AVIS:

L'auteur a accordé une licence non exclusive permettant à la Bibliothèque et Archives Canada de reproduire, publier, archiver, sauvegarder, conserver, transmettre au public par télécommunication ou par l'Internet, prêter, distribuer et vendre des thèses partout dans le monde, à des fins commerciales ou autres, sur support microforme, papier, électronique et/ou autres formats.

L'auteur conserve la propriété du droit d'auteur et des droits moraux qui protege cette thèse. $\mathrm{Ni}$ la thèse ni des extraits substantiels de celle-ci ne doivent être imprimés ou autrement reproduits sans son autorisation.
In compliance with the Canadian Privacy Act some supporting forms may have been removed from this thesis.

While these forms may be included in the document page count, their removal does not represent any loss of content from the thesis.
Conformément à la loi canadienne sur la protection de la vie privée, quelques formulaires secondaires ont été enlevés de cette thèse.

Bien que ces formulaires aient inclus dans la pagination, il n'y aura aucun contenu manquant. 


\section{To my mother}




\section{Abstract}

This thesis reports the design and development of two classes of new composite materials, which are low-carbon Stellite alloy matrices, reinforced with either chopped plain carbon fiber or chopped nickel-coated carbon fiber. The focus of this research is on obviating the problems related to the presence of carbides in Stellite alloys by substituting carbides as the main strengthening agent in Stellite alloys with the aforementioned carbon fibers. Stellite 25 was selected as the matrix because of its very low carbon content $(0.1 \mathrm{wt} \%)$ and thereby relatively carbide free microstructure. The nickel coating was intended to eliminate any chance of carbide formation due to the possible reaction between carbon fibers and the matrix alloying additions. The composite specimens were fabricated using the designed hot isostatic pressing and sintering cycles. The fabricated specimens were microstructurally analyzed in order to identify the main phases present in the specimens and also to determine the possible carbide formation from the carbon fibers. The material characterization of the specimens was achieved through density, hardness, microhardness, corrosion, wear, friction, and thermal conductivity tests. These novel materials exhibit superior properties compared to existing Stellite alloys and are expected to spawn a new generation of materials used for high temperature, severe corrosion, and wear resistant applications in various industries. 


\section{Acknowledgements}

I would like to express my sincere gratitude to my thesis supervisors, Professor R. Liu and Professor M. Liang, for their continuous support throughout this research.

I wish to thank my NRC co-supervisor Dr. Q. Yang, Dr. X. J. Wu, T. Benak, A. Merati and N. Powell for their generous help.

I also wish to thank Dr. M. X. Yao of Kennametal Stellite for technical guidance through the progress of the project.

I am also grateful to D. Morphy, D. Chow, R. MacNeil, and M. Genest for their assistance in my laboratory work at the National Research Council Canada (NRC).

Deepest thanks to my family and especially my parents who are a source of unwavering love and encouragement and my friends for their energetic support. 


\section{Table of Contents}

Abstract

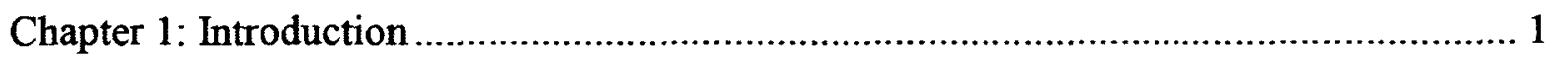

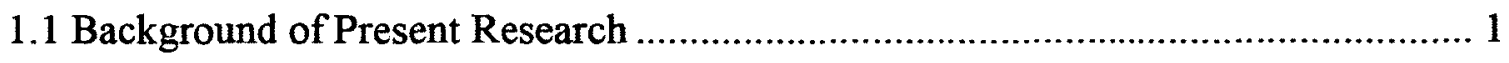

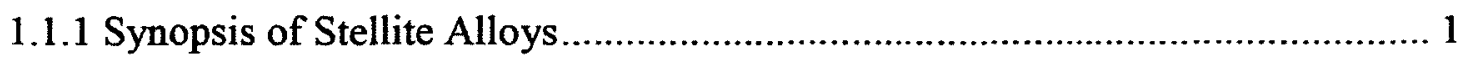

1.1.2 Chemical Compositions of Stellite Alloys ........................................ 2

1.1.3 Carbide Related Issues in Stellite Alloys ........................................... 3

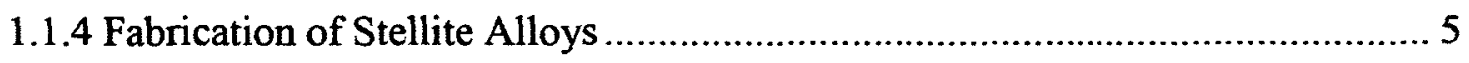

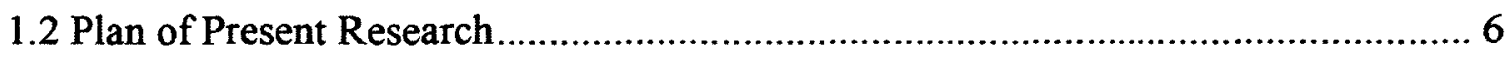

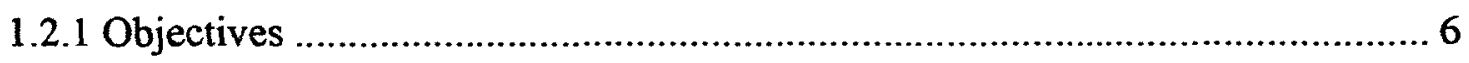

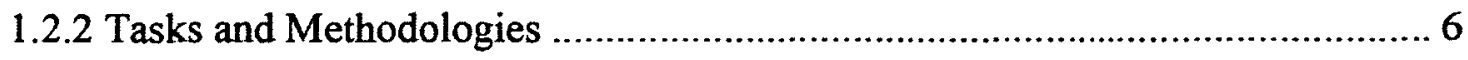

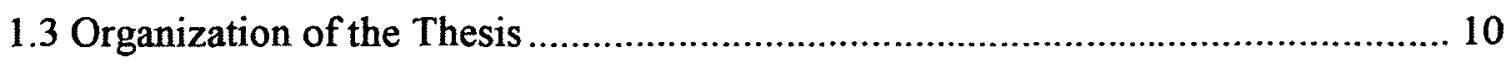




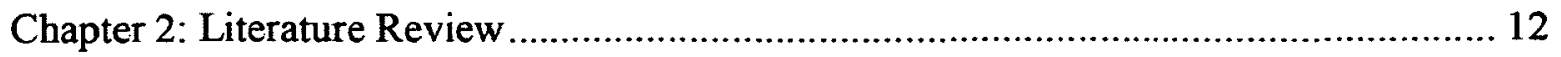

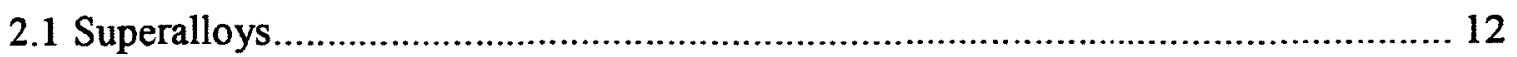

2.1.1 Fundamentals and Classification ............................................................ 12

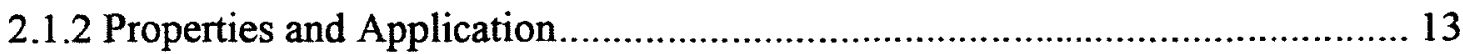

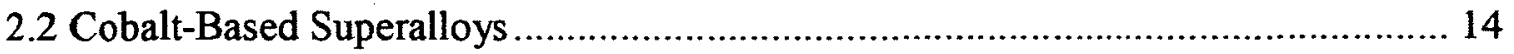

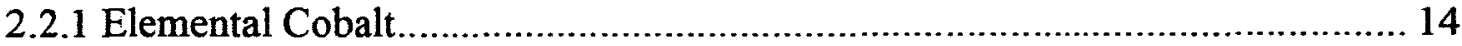

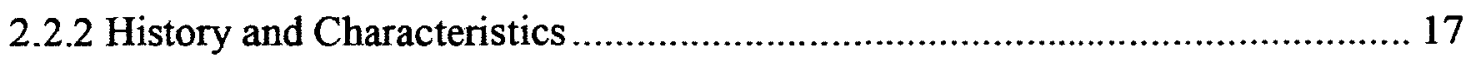

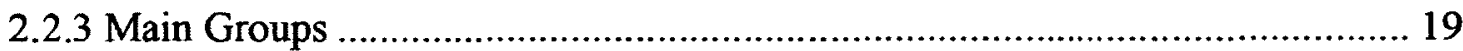

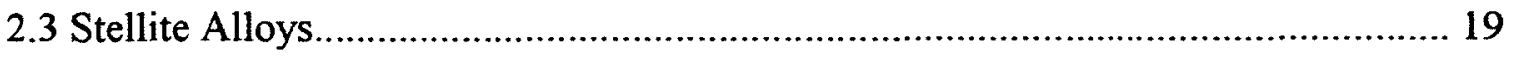

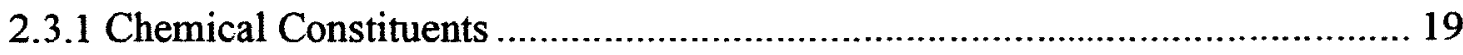

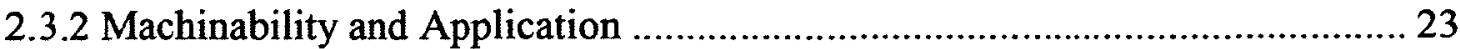

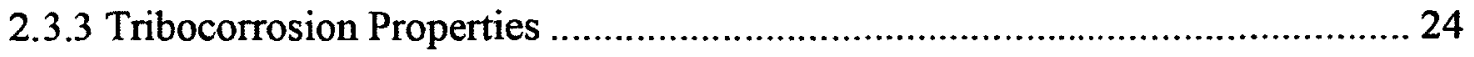

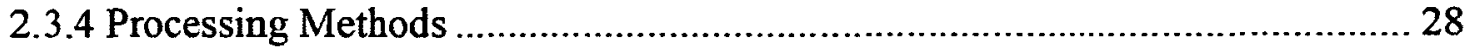

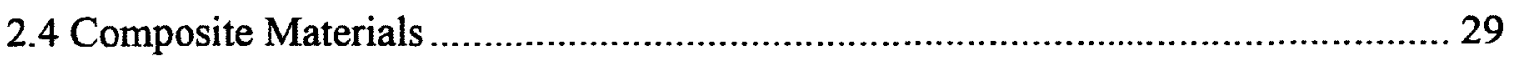

2.4.1 Types of Composite Materials ................................................................... 29

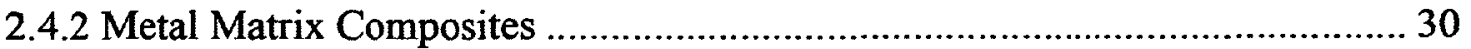

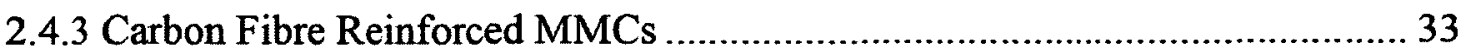

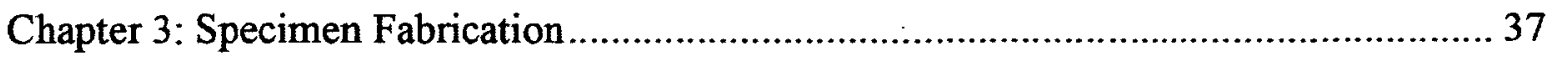

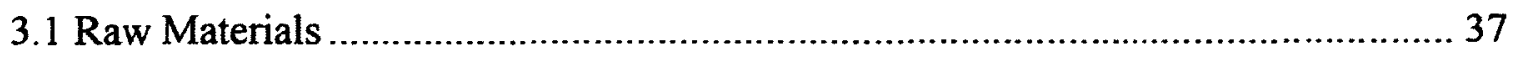

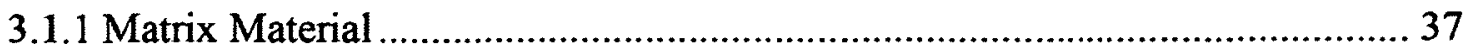

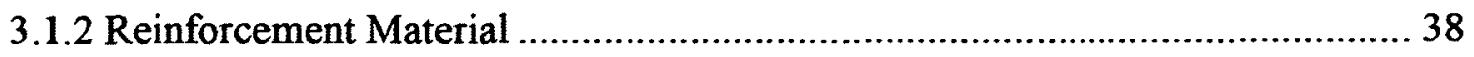

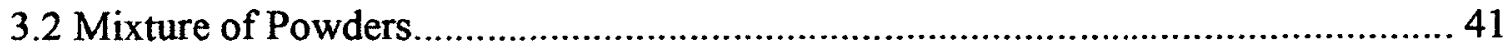


3.2.1 Chemical Composition of the Composites .................................................. 41

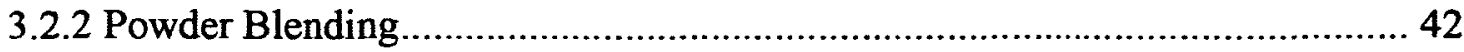

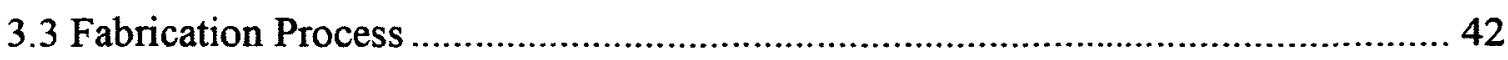

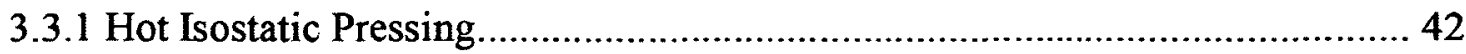

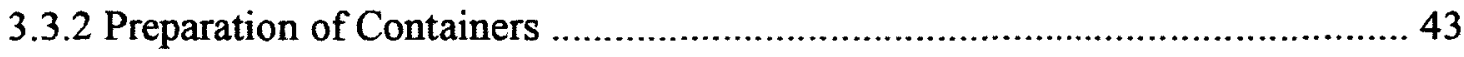

3.3.3 Differential Scanning Calorimetry ................................................................ 44

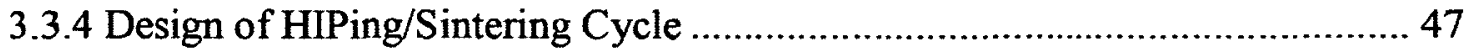

Chapter 4: Material Characterization....................................................................... 50

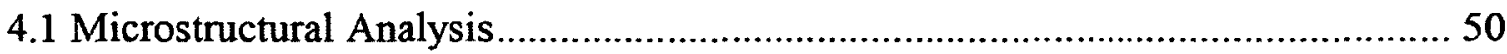

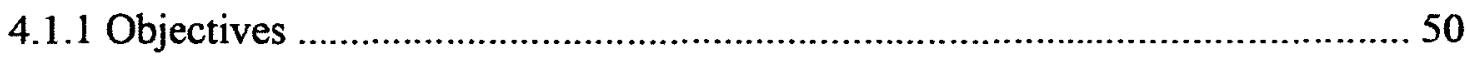

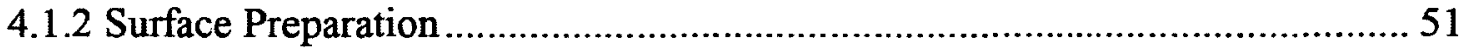

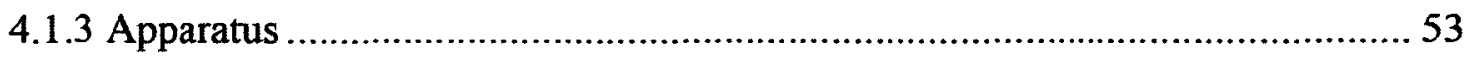

4.1.4 Carbide Volume Fraction Analysis.......................................................... 54

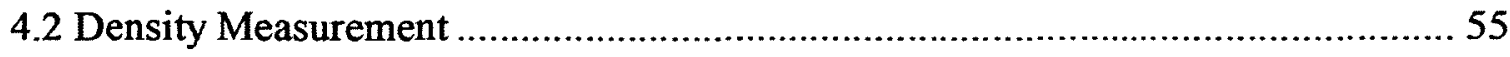

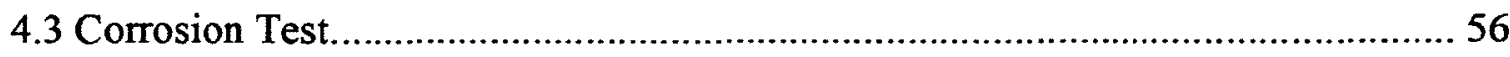

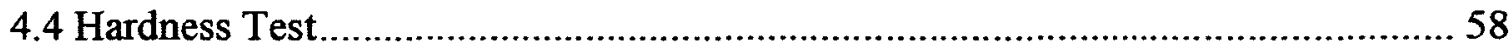

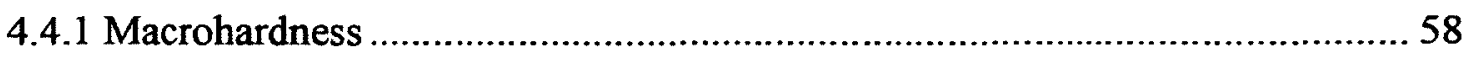

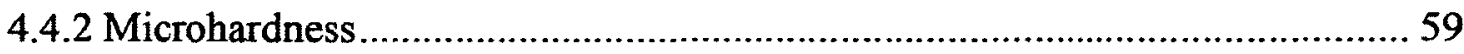

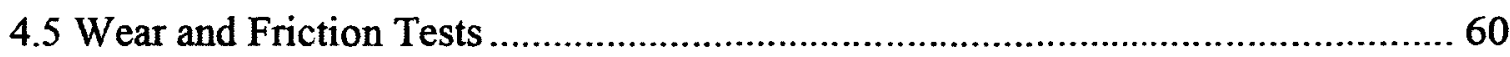

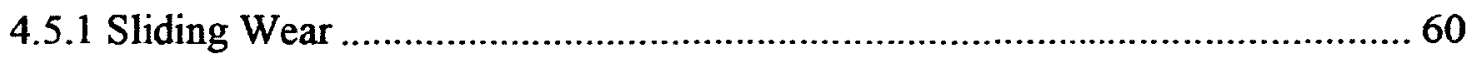

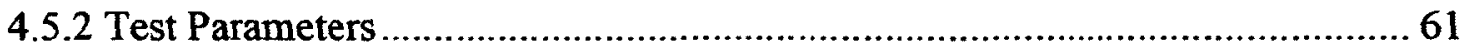

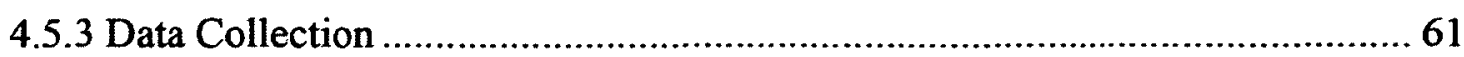




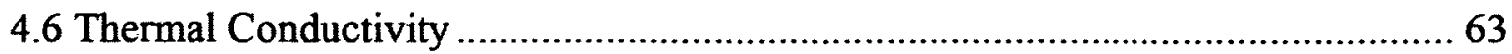

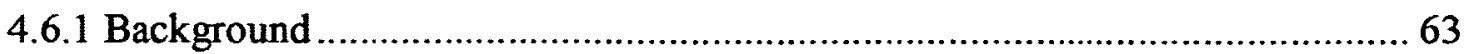

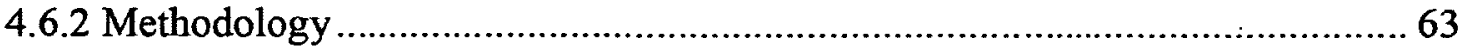

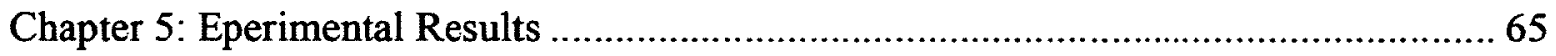

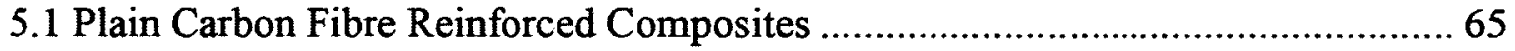

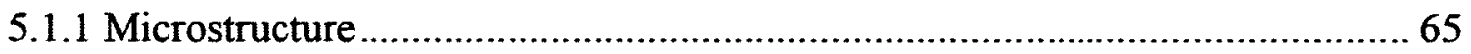

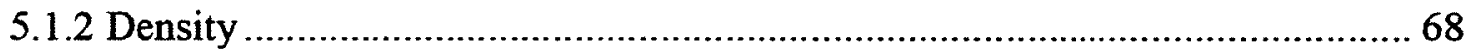

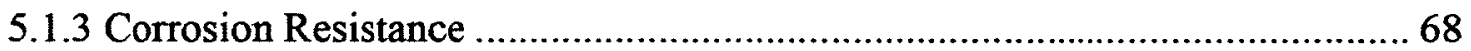

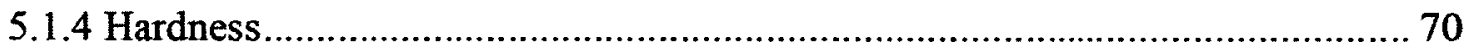

5.1.5 Friction and Wear Resistance ................................................................... 71

5.2 Ni-Coated Carbon Fibre Reinforced Composites ……………………................. 74

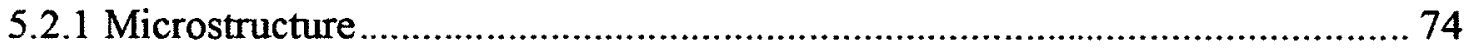

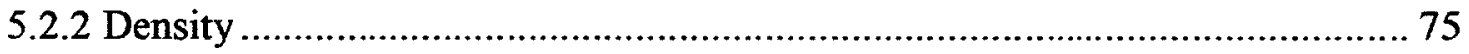

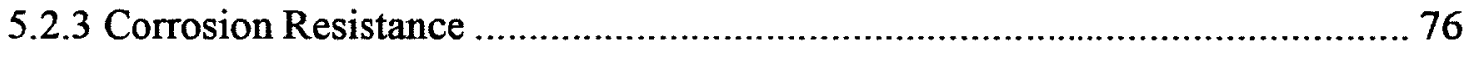

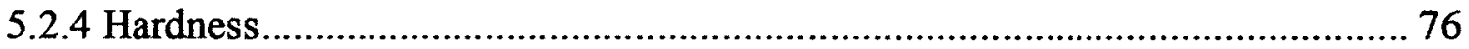

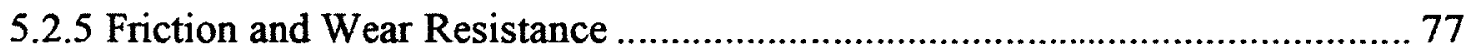

5.3 Supporting Tests Results................................................................................ 79

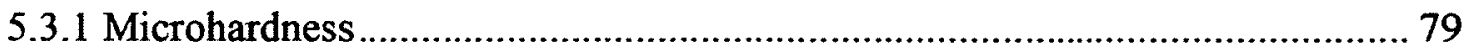

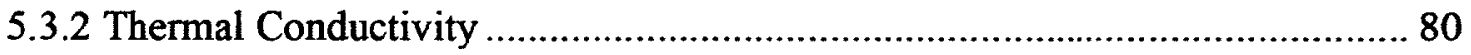

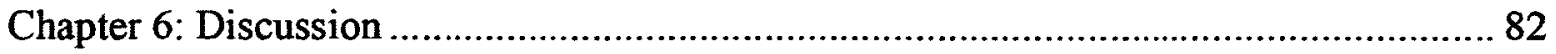

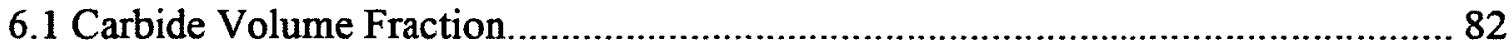




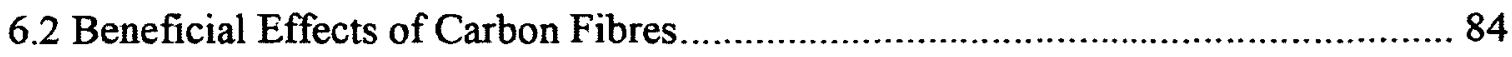

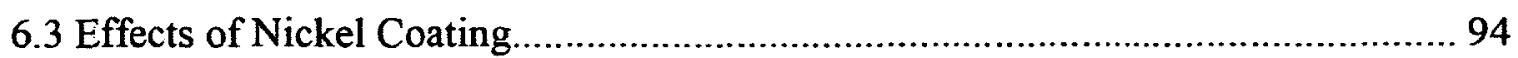

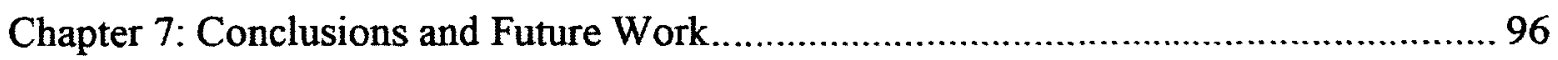

7.1 Conclusions

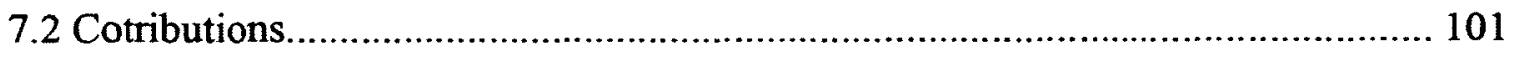

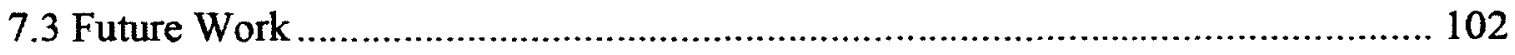

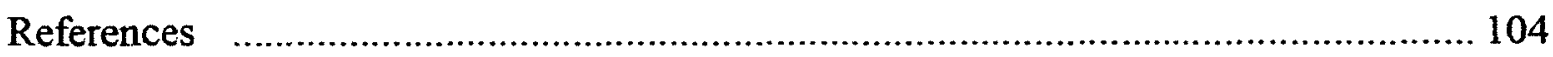

Tables

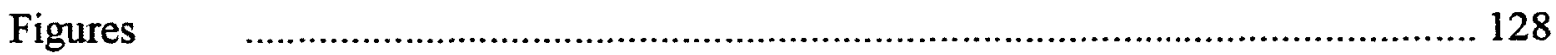




\section{List of Tables}

Table 2.1 Physical properties of elemental cobalt .......................................... 118

Table 2.2 Mechanical properties of elemental cobalt ........................................ 118

Table 2.3 Chemical compositions (wt \%) of Tribaloy alloys ............................. 119

Table 2.4 Compositions (wt \%, Co in balance) of various Stellite alloys .................. 119

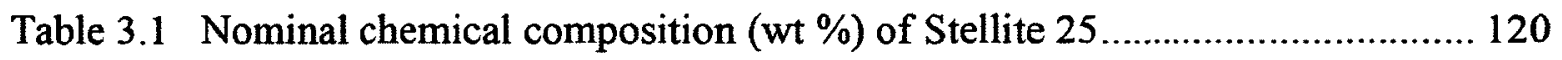

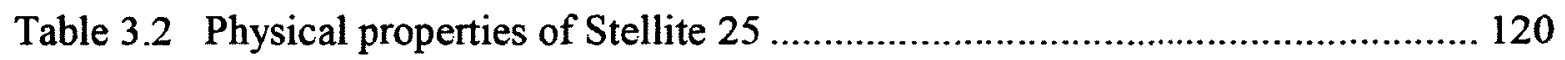

Table 3.3 Mechanical properties of Stellite 25 at room temperature ....................... 120

Table 3.4 Physical properties of chopped plain carbon fibre at room temperature ...... 121

Table 3.5 Mechanical properties of chopped plain CF at room temperature.............. 121

Table 3.6 Physical properties of chopped nickel-coated CF at room temperature ....... 121

Table 3.7 Mechanical properties of chopped nickel-coated CF at room temperature .. 122

Table 3.8 Chemical composition of Stellite 25 and Stellite 25-based composites ....... 122

Table 4.1 General properties of phenolics ................................................. 123

Table 4.2 The procedure of grinding and polishing operations ........................... 124

Table 4.3 Carbide identifying reagent and etching procedure ............................. 124

Table 5.1 Carbide volume fraction in plain CF reinforced composite specimens....... 125

Table 5.2 Specific densities of the plain CF reinforced composite specimens........... 125

Table 5.3 Carbide volume fraction in Ni-coated CF reinforced composite specimens 126

Table 5.4 Specific densities of Ni-coated CF reinforced composite specimens.......... 126 
Table 5.5 Mean microhardness values of specimen $F$, specimen $\mathrm{O}$, and their carbidic phase..

Table 5.6 Relative thermal properties obtained with pulsed thermography technique. 127

Table 6.1 Property comparison between the fabricated composites and PTFE based

sliding bearing materials 


\section{List of Figures}

Figure 2.1 Typical microstructure of Tribaloy alloys: (a) T-400 and (b) T-800 ...... 129

Figure 2.2 Typical microstructure of Stellite alloys: (a) Stellite 1, (b) Stellite 6 (cast),

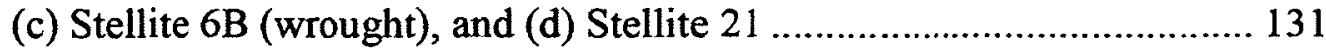

Figure 3.1 Three dimensional Turbula type T2C mixer..................................... 132

Figure 3.2 Consolidation container specimens.......................................... 132

Figure 3.3 Vacuum outgassing pump.......................................................... 133

Figure 3.4 Netzsch 404C Pegasus heat flux DSC instrument ............................. 133

Figure 3.5 DSC heating/cooling cycle ................................................... 134

Figure 3.6 DSC thermograms: (a) specimen A, (b) specimen B, (c) specimen C and (d)

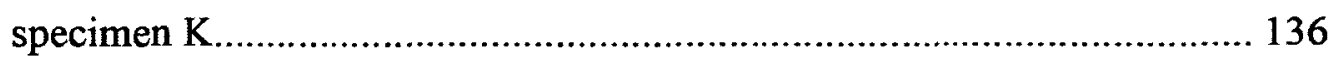

Figure 3.7 Sintering cycle for pure Stellite 25 and Stellite 25 based composites ...... 136

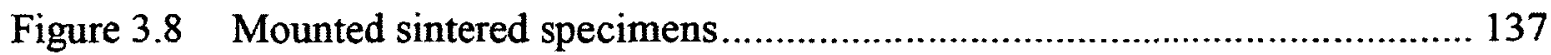

Figure 4.1 Struers Secotom-10 cutting machine ........................................... 137

Figure $4.2 \quad$ LaboPress-3 mounting press ........................................................ 138

Figure 4.3 Buehler Ecomet-4 semiautomatic grinder polisher .......................... 138

Figure 4.4 Olympus PM-63 optical microscope ............................................. 139

Figure 4.5 Hitachi S-570 scanning electron microscope................................... 139

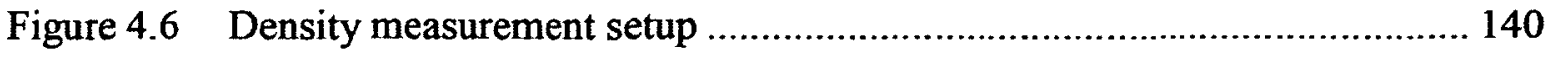

Figure 4.7 Immersion corrosion test specimens ............................................. 140 
Figure 4.8 Rockwell hardness test apparatus

Figure 4.9 SMT-X7 dual indenter microhardness tester ................................. 141

Figure 4.10 TEER-POD-2 computer controlled pin-on-disk tribometer ................... 142

Figure 4.11 Pulsed thermography test setup ................................................ 142

Figure 5.1 Optical microstructural images of the specimens in an unetched condition:

(a) pure Stellite 25 (specimen A), (b) composite specimen F and (c)

presence of voids in the composite specimen (specimen $C$ ). 144

Figure 5.2 SEM image of the etched composite F in an area close to carbon fibres .. 145

Figure 5.3 SEM/EDX analysis of specimen F: (a) SEM microstructural image at high magnification, (b) EDX spectrum of white phase, (c) EDX spectrum of dark grey phase and (d) EDX spectrum of black phase 147

Figure 5.4 Optical microstructural image of the etched pure Stellite 25 specimen .... 148

Figure 5.5 Microstructural pictures used for the evaluation of carbide volume fraction:

(a) specimen A, (b) specimen B, (c) specimen C, (d) specimen D, (e)

specimen $E$ and (f) specimen $F$

Figure 5.6 Corrosion resistances of plain carbon fibre reinforced specimens in oxidizing acid $65 \% \mathrm{HNO}_{3}$ at boiling temperature

Figure 5.7 Rockwell hardnesses of plain carbon fibre reinforced specimens 152

Figure 5.8 Variations of friction coefficient of plain carbon fibre reinforced specimens with sliding time 153

Figure 5.9 Volume losses of plain CF reinforced specimens due to sliding wear ...... 153

Figure 5.10 Specific wear rates of plain CF reinforced specimens under sliding wear 154 
Figure 5.11 Optical images of worn surfaces: (a) specimen A, (b) specimen B, (c) specimen $C$, (d) specimen $D$, (e) specimen $E$ and (f) specimen $F$

Figure 5.12 Severe wear scars in the specimen A surface 158

Figure 5.13 Intermittent nature of the wear tracks in plain CF reinforced composites. 158

Figure 5.14 Worn surface analyses of pure Stellite 25 specimen: (a) SEM morphology and (b) EDX spectrum

Figure 5.15 Worn surface analyses of composite specimen F: (a) SEM morphology and

(b) EDX spectrum 160

Figure 5.16 Worn surface analyses of carbon fibres in composite specimen F: (a) SEM morphology and (b) EDX spectrum 161

Figure 5.17 Microstructural images: (a) Unetched pure Stellite 25 (specimen A), (b) unetched Ni-coated CF reinforced composite (specimen P), (c) unetched specimen $P$ at higher magnification and (d) the matrix of etched specimen $P$ in an area close to the fibres. 163

Figure 5.18 Presence of voids in the Ni-coated carbon fibre reinforced composites.... 164

Figure 5.19 Pictures used for carbide volume fraction analysis: (a) specimen A, (b) specimen $K$, (c) specimen $L$, (d) specimen $M$, (e) specimen $N$, (f) specimen $\mathrm{O}$ and $(\mathrm{g})$ specimen $\mathrm{P}$ 167

Figure 5.20 Corrosion test results of Ni-coated CF reinforced composite specimens .. 168

Figure 5.21 Rockwell hardness test results of the Ni-coated carbon fibre reinforced composite specimens 168

Figure 5.22 Variations of friction coefficient for Ni-coated carbon fibre composite specimens with sliding time. 
Figure 5.23 Volume losses of Ni-coated carbon fibre reinforced composite specimens under pin-on-disc wear 169

Figure 5.24 Specific wear rates of Ni-coated carbon fibre reinforced composite specimens under pin-on-disc wear.

Figure 5.25 Worn surfaces: (a) specimen A, (b) specimen $K$, (c) specimen $L$, (d) specimen $M$, (e) specimen $N$, (f) specimen $O$ and (g) specimen $P$ 174

Figure 5.26 Worn surface analyses of specimen O: (a) SEM micrograph and (b) EDX spectrum 175

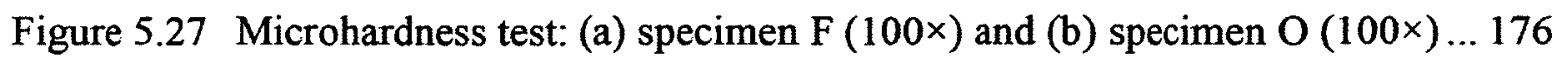

Figure 5.28 Microhardness test of the carbidic phase in specimen $\mathrm{F}(500 \times)$ 176

Figure 5.29 Thermal conductivity study: (a) tested specimens, (b) PT thermogram at 1s and (c) PT thermogram at $8.15 \mathrm{~s}$ 177

Figure 6.1 Vickers hardness indentations on specimen F under load of $60 \mathrm{~kg}$ : (a) close to the interface and (b) at the interface 178

Figure 6.2 Vickers hardness indentations on specimen $O$ under load of $60 \mathrm{~kg}$ : (a) close to the interface and (b) at the interface 179

Figure 6.3 Comparison of specific density between plain fibre reinforced composites and Ni-coated fibre reinforced composites. 180

Figure 6.4 Comparison of corrosion rate between plain fibre reinforced composites and $\mathrm{Ni}$-coated fibre reinforced composites. 180

Figure 6.5 Comparison of hardness between plain fibre reinforced composites and $\mathrm{Ni}$-coated fibre reinforced composites. 
Figure 6.6 Comparison of friction coefficient between plain fibre reinforced composites and $\mathrm{Ni}$-coated fibre reinforced composites.

Figure 6.7 Comparison of wear rate between plain fibre reinforced composites and .......

Ni-coated fibre reinforced composites.................................................. 182 


\section{Nomenclature}

\begin{tabular}{ll}
$\mu$ & \multicolumn{1}{c}{ Coefficient of friction } \\
$\gamma$ & Austenitic phase \\
$\gamma^{\prime}$ & Aluminide precipitates \\
$\gamma^{\prime \prime}$ & \multicolumn{1}{c}{ BCT $\mathrm{Ni}_{3} \mathrm{Nb}$} \\
$\delta$ & Orthorhombic $\mathrm{Ni}_{3} \mathrm{Nb}$ \\
$\eta$ & Hexagonal $\mathrm{Ni}_{3} \mathrm{Ti}$ \\
$\mathrm{E}$ & HCP cobalt \\
$\alpha$ & FCC cobalt \\
$\mathrm{K}$ & Thermal conductivity \\
$\rho$ & Density \\
W & Weight \\
$\mathrm{M}$ & Mass \\
$\mathrm{t}$ & Time \\
A & Area \\
V & Volume \\
F & Normal force \\
k & Specific wear rate \\
$\mathrm{T}$ & Temperature \\
$\mathrm{Q}$ & Applied energy \\
\hline &
\end{tabular}




\section{List of Acronyms}

\begin{tabular}{ll} 
FCC & Face centered cubic \\
HCP & Hexagonal close packed \\
BCT & Body centered tetragonal \\
P/M & Powder metallurgy \\
HIP & Hot isostatic pressing \\
CF & Carbon fibre \\
SEM & Scanning electron microscopy \\
ASTM & American society for testing and materials \\
MTS & Material testing system \\
ASM & American society for metals \\
SA & Superalloy \\
OMC & Organic matrix composite \\
CMC & Ceramic matrix composite \\
MMC & Metal matrix composite \\
PAN & Polyacrylonitrile \\
GMAW & Gas metal arc welding \\
MSDS & Material safety data sheet \\
EMI & Electromagnetic interference \\
RFI & Radio frequency interference \\
\hline
\end{tabular}

$$
\text { xix }
$$




$\begin{array}{ll}\text { DSC } & \text { Differential scanning calorimetry } \\ \text { DFRAC } & \text { Discontinuous fibre reinforced aluminum alloy composite } \\ \text { EDX } & \text { Energy dispersive X-ray } \\ \text { PT } & \text { Pulsed thermography } \\ \text { IR } & \text { Infra red } \\ \text { PTFE } & \text { Polytetrafluoroethylene } \\ \text { SFE } & \text { Stacking fault energy }\end{array}$




\section{Chapter 1: Introduction}

\subsection{Background of Present Research}

\subsubsection{Synopsis of Stellite Alloys}

Many industrial processes such as gas turbine engine operation, oil production and refining, chemical processing industry, and mechanical manufacturing pose high stress, high temperature, and severe wear and corrosion environments. Therefore, it is highly desirable to develop materials of high strength at elevated temperatures that possess good corrosion and wear resistance as well. In response to this need, superalloys (SAs) which are a group of high-performance alloys have been introduced to the market. These alloys can be divided into three major classes; iron-based superalloys, nickel-based superalloys, and cobalt-based superalloys [1].

The use of cobalt (Co) element as a constituent in cobalt-based superalloys is to provide the ductile matrix and to impart high-temperature strength $[1,2]$. Many of the properties of these alloys arise from the crystallographic nature of cobalt. This element imparts an unstable, face-centered cubic (fcc) crystal structure with a very low stacking fault energy (SFE) to its alloys. The unstable fcc structure and its associated low stacking fault energy result in high yield strength, high work-hardening rate due to the interaction between stacking faults, limited fatigue damage under cyclic stresses due to the lack of 
cell walls within plastically deformed materials, and the ability to absorb stresses through the transformation of the structure to hexagonal close packed (hcp) [1-3].

Carbon- (C) containing cobalt-based alloys can be categorized into three groups: high-carbon alloys $(1.2 \mathrm{wt} \% \sim 3 \mathrm{wt} \%)$ designed for wear service, low-carbon alloys (< $0.5 \mathrm{wt} \%)$ designed for high-temperature service, and medium $(0.5 \mathrm{wt} \% \sim 1.2 \mathrm{wt} \%)$ to low-carbon alloys designed to combat corrosion or simultaneous corrosion and wear [1]. Generally, the softer and tougher compositions are used for high-temperature applications, such as gas turbine vanes and buckets, while the harder grades are used for wear resistant materials $[4,5]$. Stellite alloys make up one main family of tribocorrosion resistant cobalt-based superalloys with various types of commercial grades that satisfy most of the industry requirements. It is believed that the properties of a Stellite alloy mostly depend upon its chemical composition and fabrication process [6].

\subsubsection{Chemical Compositions of Stellite Alloys}

In the case of the chemical composition, in addition to carbon, the main constituents of Stellite alloys include chromium (Cr), tungsten (W), molybdenum (Mo), nickel (Ni), iron (Fe), Aluminum (Al), etc. Each constituent plays a different role in Stellite alloys. For example, chromium mainly implants corrosion resistance and forms carbides, tungsten strengthens the solid solution matrix, molybdenum functions same as tungsten but also enhances the wear resistance. The contents of these constituents influence the final properties of these alloys. 
The effects of the main strengthening phases in Stellite alloys have been recognized. In these alloys, carbides are considered as the primary strengthening phase and solid-solution hardeners including chromium, tungsten, and molybdenum are known as the secondary strengthening phases.

\subsubsection{Carbide Related Issues in Stellite Alloys}

Regarding the primary strengthening phase, one of the key issues is the carbon content in the alloys. In other words, the carbon content and, thus, carbide volume fraction in the material, is considered as the main distinction among individual Stellite alloys. In these alloys, carbides can be found in various forms, including $M C, M_{2} C$, $\mathrm{M}_{7} \mathrm{C}_{3}, \mathrm{M}_{23} \mathrm{C}_{6}, \mathrm{M}_{6} \mathrm{C}, \mathrm{M}_{12} \mathrm{C}$ and $\mathrm{M}_{28} \mathrm{C}$, where $\mathrm{M}$ stands for different metals [1,4-6]. Although many types of carbides can improve the mechanical and tribological properties of Stellite alloys, the presence of carbon in these alloys has its own problems.

Firstly, carbon existence in Stellite alloys may lead to the formation of some types of unwanted intermetallic compounds and/or carbides that are also detrimental with respect to tribomechanical properties. For instance, $\mathrm{M}_{2} \mathrm{C}$ and $\mathrm{M}_{6} \mathrm{C}$ carbides are brittle and decrease the mechanical strength of Stellite alloys [7]. Also, some MC carbides such as tungsten carbide (WC) have a low heat of formation which makes them easily dissolved in high-temperature wear conditions [8]. This limits the application of superalloys where a combination of high toughness and high hardness is needed. Secondly, when subjected to a high-temperature cyclic stress, carbidic phases $M_{7} C_{3}$ and MC are oxidized preferentially, which creates crack initiation sites in Stellite alloys; thus 
inevitably undermines certain tribomechanical properties of the alloys [9]. Thirdly, carbide transformation is a common phenomenon during fatigue or service at high temperatures. For instance, $\mathrm{M}_{7} \mathrm{C}_{3}$ and $\mathrm{MC}$ carbides are metastable and decompose sluggishly during cyclic loading to form a large amount of $\mathrm{M}_{23} \mathrm{C}_{6}$ carbide; resulting in an inhomogeneous distribution of $\mathrm{M}_{23} \mathrm{C}_{6}$ in the vicinity of the primary carbides $[9,10]$. Likewise, the solidifying $\mathrm{M}_{6} \mathrm{C}$ carbide degenerates over a certain concentration range of chromium at elevated temperatures, causing secondary WC formation. Furthermore, along with the carbide types, different sizes and shapes of the carbide particles within Stellite alloys may also strongly influence the properties of the alloys. For instance, larger carbides that usually pose higher strength provide the alloys with higher resistance to wear $[1,3]$.

As a result, the problems associated with the presence of carbon in Stellite alloys can be summarized as formation of unwanted intermetallic compounds and/or carbidic phases, oxidation of carbides, transformation of carbides, and inhomogeneous distribution of carbidic phases. Unfortunately, the presence of unwanted intermetallic compounds and carbidic phases in Stellite alloys is inevitable and the type, size, shape, distribution, and amount of desired carbides cannot be easily predicted or controlled. This is due to the fact that the carbide formation process in Stellite alloys is affected by many factors, including alloying element types and fraction, carbon content, fabrication technique, heating and cooling rates, operational parameters, etc. 


\subsubsection{Fabrication of Stellite Alloys}

Stellite materials can be produced using different methods of fabrication such as casting, wrought, and powder metallurgy ( $\mathrm{P} / \mathrm{M})$. Comparing these three fabrication methods, $\mathrm{P} / \mathrm{M}$ is considered as an efficient and versatile method for manufacturing Stellite materials with near-net-shapemaking capability, as well as less chemical partitioning and microsegregation problems $[1,11]$. In view of the success of $\mathrm{P} / \mathrm{M}$ techniques for different materials, it is disappointing that relatively little research effort has been made so far on the P/M fabrication of Stellite alloys. In particular, no research in P/M fabrication of carbon fibre reinforced Stellite alloy composites has been found.

Among different $\mathrm{P} / \mathrm{M}$ techniques, hot isostatic pressing (HIP) is known as an effective fabrication process. The advantages of HIP over other P/M techniques include the capability of achieving high-density materials with isotropic physical/mechanical properties and also improved mechanical properties [11]. It is believed that the HIP fabrication parameters, including sintering temperature, sintering heating and cooling rates, HIP maximum pressure, and HIP time length have considerable effects on the final properties of the P/M Stellite materials. Therefore, a properly designed HIPing/sintering cycle is required to ensure that the final products meet the requirements and can be effectively put into operation. 


\subsection{Plan of Present Research}

\subsubsection{Objectives}

The present research was a joint project between Carleton University and the National Research Council Canada (NRC), sponsored by an NSERC Collaborative Research and Development Grant (CRD) program with the Deloro Stellite Group as the industrial collaborator. The specimen fabrication, microstructural analysis, and experimental work in this research were performed in the NRC Institute for Aerospace Research (NRC-IAR Ottawa), the Department of Mechanical and Aerospace Engineering of Carleton University, and the Department of Civil and Environmental Engineering of Carleton University. The proposed research attempted to design and develop two classes of new materials, which are Stellite alloy based composites reinforced by carbon fibres (CFs). These new materials exhibited superior properties to conventional Stellite alloys by solving the carbide problems that exist in Stellite alloys. This can be achieved by using carbon fibres as a novel strengthener in Stellite alloys instead of different types of carbides. Thus, the fabricated composites are expected to spawn a new generation of materials used for high temperature, severe corrosion, and wear resistant applications in various industries.

\subsubsection{Tasks and Methodologies}

The new materials were fabricated by the HIP/sintering technique. To obtain the materials in good quality, the HIP parameters and sintering cycle elements of the 
fabrication process were examined to achieve an optimal HIP/sintering condition. The detailed tasks are summarized below:

\section{Part 1: New material development}

In order to eliminate the problems associated with the presence of carbides in Stellite alloys, it was attempted to substitute carbides, which are the main strengthening phase in Stellite alloys, with carbon fibres. Using the idea of metal matrix composites (MMCs) and their inherent advantages with respect to other types of composites and unreinforced metals, carbon fibres, which possess very useful engineering properties, were employed as reinforcing elements in Stellite alloys. The functional engineering properties of carbon fibres, which make them good replacements for carbides in Stellite alloys, include their low density, excellent thermal stability, high chemical inertness, good mechanical properties and outstanding thermal conductivity along with their broad commercialization. In the newly fabricated composites, a special low carbon Stellite alloy designated as Stellite 25, with the main alloying elements of chromium, tungsten and nickel, was selected as the matrix material. The reinforcing elements included chopped plain carbon fibres and chopped nickel coated carbon fibres with $3 \mathrm{~mm}$ and $6 \mathrm{~mm}$ lengths respectively. The use of the chopped form of carbon fibres can promote the fabrication of randomly oriented fibre reinforced-composites which exhibit isotropic properties. In this phase, pure Stellite 25 material along with two groups of composite materials consisting of eleven different Stellite 25 based composites with 0.25 to $10 \mathrm{wt} \%$ of the two types of carbon fibres were fabricated. The fabrication process included the following steps: 
1. Material blending: Mixing Stellite 25 powder with carbon fibres using a three dimensional mixer to produce a homogeneous distribution of carbides.

2. Vacuum outgassing: Placing the blended materials in HIP containers (round tubes of stainless steel-grade 316 ) followed by outgassing and sealing the containers.

3. Differential scanning calorimetry (DSC) testing: Studying the phase formation and transformation temperatures of the blended materials using the DSC thermograms as a quality control method to evaluate the purity of Stellite 25 powder and also as a basis for designing of the sintering cycle.

4. Designing the HIP/sintering cycles: Selecting HIP parameters and sintering cycle elements including HIP pressure and time along with sintering peak temperature, hold periods, heating and cooling rates.

5. Fabricating specimens: Subjecting the sealed HIP containers to the designed P/M route by simultaneously applying the set isostatic pressure to the containers and consolidating the powders and reinforcements with the designed sintering cycle.

\section{Part II: Microstructural analysis}

The fabricated specimens were microstructurally analyzed in order to ensure that the fabrication parameters were selected properly and also to identify the main phases present in the specimens. The details included the following:

1. Surface preparation: The surfaces of the specimens were prepared in several steps including cutting, mounting, grinding, polishing, and etching. 
2. Optical microscopy: The microstructural images of the specimens were obtained with an Olympus PM-63 light microscope.

3. Scanning electron microscopy (SEM): The micrographs of the microstructural features of the specimens were acquired by a Hitachi S-570 model SEM.

4. Energy dispersive X-ray (EDX): The EDX spectra were employed to analyze the chemical characterization of the specimens.

5. Carbide volume fraction analysis: An image analysis software package, Clemex Vision Lite ${ }^{\mathrm{TM}}$, was employed to examine whether carbon fibres induce any carbides in the alloy matrix.

\section{Part III: Material characterization}

Various properties of the newly developed composites were characterized as described below:

1. Density test: The density of each specimen was measured using immersion density technique in accordance with the ASTM Standard B 311-08 [12].

2. Corrosion test: Immersion testing method was used to evaluate the corrosion resistance of the specimens according to the ASTM Standards G 1-03 [13] and G 31$72[14]$.

3. Hardness test: Overall hardness of each specimen was determined with a Rockwell hardness tester according to the ASTM Standard E 18-08b [15].

4. Microhardness test: Hardness variation from the interface between the matrix and carbon fibres to the matrix of each fabricated composites was measured using a fully- 
automated MMT-X7 dual indenter microhardness tester provided by Clemex Technologies Inc. The purpose of this test was to investigate whether any diffusion of carbon from the fibres into the matrix occurred during the material fabrication process. If the diffusion occurred, the hardness of the interface region would increase due to the formation of new carbides.

5. Wear test: A computer controlled pin-on-disk tribometer was employed to evaluate the wear resistance of each specimen in accordance with ASTM Standard G 99-05 [16]. The worn surfaces of all the specimens were studied using optical and SEM microscopies and the EDX technique was used to analyze the chemical characterization of the worn surfaces.

6. Friction test: The coefficient of friction $(\mu)$ of each specimen was automatically recorded throughout the wear test with the aid of a linear variable displacement transducer.

7. Thermal conductivity test: The variation in thermal conductivity due to the incorporation of carbon fibres into Stellite 25 alloy was analyzed based on the Pulsed Thermography (PT) method using a $240 \times 320$ pixels infra red (IR) camera.

\subsection{Organization of the Thesis}

The thesis consists of seven chapters with the contents outlined as follows:

Chapter 1 is an introduction to this research, which describes the background and significance, as well as the objectives and methodologies of this research. 
Chapter 2 is the literature review of the previous studies related to this research.

Chapter 3 details the specimen fabrication methods and procedures such as "Green body" preparation and HIPing/sintering processes.

Chapter 4 describes the material characterization tests including microstructural analysis, density test, hardness test, tribological tests, and corrosion test.

Chapter 5 presents the experimental results.

Chapter 6 provides the discussion of the obtained results.

The conclusions drawn from this research along with the recommendations for future work are given in Chapter 7. 


\section{Chapter 2: Literature Review}

\subsection{Superalloys}

\subsubsection{Fundamentals and Classification}

The term superalloy is used to describe nickel-, iron-nickel-, and cobalt-based alloys generally used when operating temperatures are beyond about $540^{\circ} \mathrm{C}\left(1000^{\circ} \mathrm{F}\right)$, hence also known as high-performance alloys. Over the years, a large number of superalloys have been developed and used. The base alloying elements of these alloys are transition metals with consecutive positions in the periodic table of elements [1]. The matrixes of superalloys include the austenitic $\gamma$ phase with the face-centered cubic crystal structure. The secondary phase of superalloys consists of a variety of compounds which control the properties of superalloys.

In reality, superalloys are either solid solution strengthened alloys or precipitation hardened alloys. While the former alloys are strengthened both by solid solution hardening and by the presence of carbides, the latter alloys are strengthened by the combination of precipitates, solid solution hardening, and the presence of carbides or intermetallic compounds - Laves phase $[1,3,17]$. The secondary phase in cobalt basedsuperalloys generally consists of different types of carbides. In nickel- and iron-nickelbased superalloy; however, the secondary phase include various carbides, $\gamma^{\prime}$ fcc 
$\mathrm{Ni}_{3}(\mathrm{Al}, \mathrm{Ti})$ compound, $\gamma^{\prime \prime}$ body-centered tetragonal (bct) $\mathrm{Ni}_{3} \mathrm{Nb}$ compound, $\delta$ orthorhombic $\mathrm{Ni}_{3} \mathrm{Nb}$ intermetallic compound, and $\eta$ hexagonal $\mathrm{Ni}_{3} \mathrm{Ti}$ compound [1].

\subsubsection{Properties and Application}

The crucial characteristics of all types of superalloys include superior tribological properties, excellent mechanical strength, outstanding resistance to loading under static, fatigue, and creep conditions; good surface stability, superb oxidation and corrosion resistance, and good phase stability at high temperatures $[18,19]$. The incipient melting temperatures for cobalt-based superalloys are generally greater than those for nickel- and iron-nickel-based superalloy [1]. The properties of superalloys can be controlled by processing (including heat treatment) and by adjustments in composition.

Superalloy materials can be produced in cast, wrought, and powder-processed forms depending on the application/composition involved. Appropriate compositions of all superalloy-based metals can be formed into a variety of shapes such as disk, sheet, plate, bar, shaft, tubing, airfoil, surface coating, and pressure vessel (case) $[1,20]$. Typical applications of superalloys are in aircraft, marine and industrial gas turbines, space vehicles, nuclear reactors, submarines, petrochemical production, chemical processing vessels, aircraft skins, power generation turbines, dental and orthopedic prostheses, military electric motors, cutting tools, environmental protection applications, and heat exchanger tubing. While high-temperature usage remains the predominant application on a volume basis, superalloys are also employed at cryogenic temperatures and at body 
temperature. Since the application of components fabricated from the superalloys requires very conservative designs, the occurrence of failure is quite rare $[1,18,20]$.

\subsection{Cobalt-Based Superalloys}

\subsubsection{Elemental Cobalt}

Cobalt is a tough, lustrous, silvery-gray ferrous metal that resembles iron and nickel in appearance and in some properties. The element with atomic number 27 , cobalt (symbol Co) falls between iron and nickel in the middle of the ferromagnetic trio, which form the transition group VIII of the first long period of the periodic table [2,4]. Although cobalt-based compounds had been used for many centuries for making jewelry and paints, isolation of the free metallic cobalt was first effected by the Swedish chemist Georg Brandt in 1735 [21]. Pure cobalt is not found as a native metal in nature; however, it occurs in various metallic-lustered ores in a fairly widespread but dispersed form. The main ores of cobalt are sulfidic cobaltite (CoAsS), safflorite $\left(\mathrm{CoAs}_{2}\right)$, skutterudite $\left(\mathrm{CoAs}_{3}\right)$, and smaltite $\left(\mathrm{CoNiAs}_{3}\right)$. Cobalt is not usually mined alone but is produced as a byproduct of other more abundant or more valuable metals, particularly copper, nickel, and platinum $[1,21]$.

Cobalt exhibits two allotropic modifications, a hexagonal close-packed structure ( $\varepsilon$-cobalt) stable at room temperatures below $417^{\circ} \mathrm{C}\left(783^{\circ} \mathrm{F}\right)$, and a face-centered cubic structure ( $\alpha$-cobalt) stable at higher temperatures between $417^{\circ} \mathrm{C}\left(783^{\circ} \mathrm{F}\right)$ and its melting point of $1493{ }^{\circ} \mathrm{C}\left(2719^{\circ} \mathrm{F}\right)$. The phase transformation temperature depends critically on the rate of temperature change, experimental conditions, and cobalt purity. While 
allotropic transformation of pure cobalt occurs at a fixed temperature as described above, alloy transformation happens gradually over a temperature range with both phases present in the intervening field. In reality, the transformation is known to be sluggish. This is due to the fact that the structure transformation free-energy change is low, about $360 \mathrm{~J} \mathrm{~mol}^{-1}$ for $\alpha \rightarrow \epsilon$ and $500 \mathrm{~J} \mathrm{~mol}^{-1}$ for $\epsilon \rightarrow \alpha$. Therefore, most cobalt-based alloys exhibit a metastable fcc structure at room temperature. Also, the grain size favors the cubic form; thus, cobalt powders, evaporated thin films, and fibres might maintain an essentially cubic phase at room temperature. The mechanism associated with the allotropic transformation is martensitic and proceeds by dislocation movements on the octahedral planes of the cubic lattice. This accounts for the plane change from $a b c a b c$ characteristic of the cubic form to $a b a b a b$ of the hexagonal form because of the stacking sequence on these planes $[1,2]$.

Elemental cobalt has an atomic weight of $58.9332 \mathrm{~g} \cdot \mathrm{mol}^{-1}$. The metal has one stable-naturally occurring isotope, $\mathrm{Co}^{59}$, and twelve other radioactive isotopes ranging in mass from 54 to 64 . The physical and mechanical properties of cobalt may be critically dependent on the crystal structures resulting from the sluggishness of the cubic-tohexagonal transformation. These properties are also influenced markedly by the effects of trace impurities and by the thermomechanical history of the material. In particular, directional properties are susceptible to the crystal orientation in the hexagonal polymorph of cobalt $[1,2]$. Table 2.1 and Table 2.2 respectively quote the physical and mechanical properties of the highest purity metal available. 
Massive cobalt is resistant to many mild corrosive agents and is very slowly attacked by air or water at temperatures below about $300^{\circ} \mathrm{C}\left(572{ }^{\circ} \mathrm{F}\right)$. However, fine cobalt powder can be much more readily attacked and may be even pyrophoric in air. Compared with nickel, cobalt is much less oxidation resistant over a wide temperature range. The oxide scale, however, progressively reduces the oxidation rate as it grows in thickness $[2,21]$.

Cobalt is considered as an important and strategic metal due to its applications in defense-related industries and the need of industrialized nations on cobalt imports. The pure metal has few applications such as scientific studies, particularly because of its allotropic transformation in different temperatures, its ferromagnetic properties with a uniquely high Curie point, and its low stacking-fault energy. The wide range of practical applications of cobalt involves its use as a chemical compound or as an alloying constituent $[1,2]$.

In general, the end uses of cobalt can be classified as nonmetallurgical and metallurgical. Nonmetallurgical applications include employing cobalt compounds in chemicals (for instance, paint pigments, catalysts for the petroleum and chemical industries, adhesives; drying agents for paints, varnishes, and inks, soaps, and ground coats for porcelain enamels) and electronic applications (for example, batteries and magnetic recording media) $[1,4]$. Cobalt compounds are also important for metallurgical applications, such as wear resistant, heat resistant, and corrosion resistant cobalt-based alloys, high-speed tool steels, nickel-based superalloys, iron-based superalloys, magnets, 
cemented carbides, high-strength alloys, low-expansion alloys, and orthopedic implants $[1,4]$.

\subsubsection{History and Characteristics}

Cobalt-based alloys were first investigated by Elwood Haynes at the turn of the $20^{\text {th }}$ century [22]. Initially, the stainless nature and high strength of the binary cobaltchromium alloys were discovered by him. Later, Haynes introduced the Co-Cr-Mo and Co-Cr-W ternaries as he discovered the powerful strengthening effects of molybdenum and tungsten within the cobalt-chromium system. Because of their star-like luster, the alloys were named Stellite after the Latin, stella, for star. The name is now a trademark of the Deloro Stellite Company, Goshen, USA. During World War I, Stellite alloys were used as cutting tool materials because of their high strength at elevated temperatures. Later, from about 1922 cobalt-based alloys were promoted in weld overlay form to protect surfaces from wear. To satisfy the need for suitable high-temperature corrosion resistant application materials, cobalt-based corrosion resistant alloys were developed in the 1930s and early 1940s. The biocompatible, low-carbon content cobalt-based alloys have been used lately as dental materials and surgical implants [22].

As a group, cobalt-based superalloys are important industrial materials with excellent high-temperature corrosion resistance. In addition, these alloys display unique combinations of mechanical and tribological properties such as high hardness and strength, good stress rupture, low creep rates, superior adhesive and abrasive wear resistance as well as excellent cavitation-erosion resistance at elevated 
temperatures $[17,23]$. The chemical composition of the cobalt based superalloys is typically comprised of about $50-60 \%$ cobalt, $\mathrm{Co}, 20-30 \%$ chromium, $\mathrm{Cr}, 5-15 \%$ tungsten, $\mathrm{W}$, or molybdenum, Mo, and $0.1-3 \%$ carbon [1].

Because of their relatively high chromium content, Co-based SAs maintain superior hot corrosion, sulfidation, and oxidation resistance at elevated temperatures compared with Ni-based SAs [20]. The cobalt-based superalloys gain their super strength by a combination of carbides as the primarily strengthening phase and solid-solution hardeners including chromium, tungsten, and molybdenum. No intermetallic compound such as aluminide precipitates $(\gamma)$ has been found for cobalt based superalloys that results in a large strength increase with the same degree of utility in nickel- or ironnickel-based superalloys $[1,10,24]$.

Many properties of the alloys originate from the crystallographic nature of cobalt. In particular, the good response to stress of this class of materials is due to the low stacking-fault energy of both cobalt allotropes [3]. Also, the fcc matrix of cobalt-based superalloys has excellent ductility and extended solubility for some alloying additions. As discussed before, the structure of the Co-rich matrix of the alloys is generally fcc, even at ambient temperature, due to the sluggish nature of the phase transformation. In addition, cobalt has a higher incipient melting temperature than nickel. Thus, amongst all types of superalloys, the cobalt-based SAs normally show greater resistance to softening at high temperatures [1]. 


\subsubsection{Main Groups}

Tribaloy alloys and Stellite alloys are known as the two main groups of cobaltbased superalloys possessing excellent high temperature properties designed for wear/corrosion resistant applications. Tribaloy alloys are a series of wear resistant cobalt(or nickel-) based alloys containing a large volume fraction of a hard, intermetallic Laves phase dispersed in a much softer eutectic matrix (Fig. 2.1) [1]. The Laves phase in cobaltbased Tribaloy alloys is a hexagonal $\mathrm{MgZn}_{2}$ structure whose composition is approximately $\mathrm{CoMoSi}$ or $\mathrm{Co}_{3} \mathrm{Mo}_{2} \mathrm{Si}$. It is due to the presence of the Laves phase in a range of $30 \sim 70$ vol\% that these materials can withstand wear under dry or boundary lubricating conditions [25]. The main alloying elements in Tribaloy alloys include molybdenum and chromium. Silicon is a minor $(\sim 3 \mathrm{wt} \%)$ constituent of Tribaloy alloys, but molybdenum and silicon are added at levels in excess of their solubility limit in order to induce the precipitation of the Laves phase [1]. The chemical compositions of commonly used Tribaloy alloys are listed in Table 2.3 [1]. Stellite alloys are a range of cobalt-chromium alloys which may also contain tungsten or molybdenum and a small $(<$ $3 \mathrm{wt} \%$ ) amount of carbon [1]. These alloys are explained in more detail below.

\subsection{Stellite Alloys}

\subsubsection{Chemical Constituents}

Stellite alloys are a range of cobalt-based materials which are derived from the Co-Cr-W-C family, first developed by Elwood Haynes, mainly for wear applications involving elevated temperatures [3]. Stellite alloys also have non-corrosive and nonmagnetic properties with astounding hardness and toughness [7]. Typical applications of 
the alloys are cutting tools, jet engine turbine blades, poppet valves, and high corrosion resistant machine parts $[19,26]$.

The principal constituents of Stellite alloys belong to the quaternary systems Co$\mathrm{Cr}-\mathrm{W}-\mathrm{C}$ or $\mathrm{Co}-\mathrm{Cr}-\mathrm{Mo}-\mathrm{C}$. These alloys are generally strengthened by the precipitation of carbides in the cobalt matrix. The compositions of various Stellite alloys are listed in Table 2.4 [1]. The main differences among the current Stellite alloy grades are their carbon and tungsten contents which affect the type and amount of carbide formation in the microstructure [1]. Considering the application of the alloys, some other elements such as nickel, molybdenum, iron, tantalum, and niobium with different weight percentages can be also added $[27,28]$. The typical microstructures of Stellite alloys, which normally consist of complex wear-resistant carbides dispersed in a tougher and more ductile cobalt solid solution mainly containing chromium and tungsten or chromium and molybdenum, are presented in Fig. 2.2. The nominal composition of these alloys are selected in accordance with the type of wear encountered (e.g., abrasive, adhesive, or erosive wear) in a particular application [1]. The major alloying constituents of Stellite alloys and their functions in the alloys are described below:

- Carbon: The density of carbon is somewhere between 1.8 and $2.1 \mathrm{~g} / \mathrm{cc}(0.065$ and $\left.0.075 \mathrm{lb} / \mathrm{in}^{3}\right)$ and its melting temperature is about $3527^{\circ} \mathrm{C}\left(6381^{\circ} \mathrm{F}\right)$. This element is inevitably present in Stellite alloys with an approximate range from 0.1 to $3.3 \mathrm{wt} \%[1,22]$. From a metallurgical point of view, carbon content determines the amount of carbides in the alloys. For instance, at a carbon level of $1 \mathrm{wt} \%$ 
(Stellite $6 \mathrm{~B}$ ), the carbides constitute about $13 \mathrm{wt} \%$ of the alloy while with a carbon content of $2.4 \mathrm{wt} \%$ (Stellite 3), the carbides constitute approximately 30 $w t \%$ of the material [1]. In general, there are four main classes of carbides: $M_{7} C_{3}$, $\mathrm{M}_{23} \mathrm{C}_{6}, \mathrm{M}_{6} \mathrm{C}$, and $\mathrm{MC}$. As discussed before, in $\mathrm{M}_{7} \mathrm{C}_{3}$ and $\mathrm{M}_{23} \mathrm{C}_{6}$ carbides $\mathrm{M}$ corresponds to $\mathrm{Cr}$; whereas in $\mathrm{M}_{6} \mathrm{C}, \mathrm{M}$ stands for $\mathrm{W}$ or $\mathrm{Mo}$. Also, In $\mathrm{MC}$ carbides, $\mathrm{M}$ represents either $\mathrm{W}$ or the reactive metals such as titanium, niobium, tantalum, and zirconium $[1,17,29]$. The type, size and shape of carbides in Stellite alloys are strongly influenced by their fabrication parameters and subtle chemistry changes. The carbides in the quaternary system Co-Cr-W-C are mostly of the chromiumrich $\mathrm{M}_{7} \mathrm{C}_{3}$ type and in alloys with high tungsten content like Stellite 1 the tungsten-rich $\mathrm{M}_{6} \mathrm{C}$ carbide is also present [1]. Since metal carbides are generally wear resistant, they improve tribological properties of the alloys and more specifically their wear resistance. Carbides formed in Stellite alloys may also influence mechanical properties such as hardness, tensile strength, and fatigue strength $[3,7,20]$. Alloying addition of carbon also promotes the stability of the face-centered cubic allotrope of the cobalt matrix in Stellite alloys even at ambient temperature [6].

- Chromium: With a range from approximately 18 to $32 \mathrm{wt} \%$, chromium is the most important alloying element in the cobalt matrix [1]. The density of $\mathrm{Cr}$ is 7.19 $\mathrm{g} / \mathrm{cc}\left(0.259 \mathrm{lb} / \mathrm{in}^{3}\right)$ where its melting point is recorded at $1907^{\circ} \mathrm{C}\left(3465^{\circ} \mathrm{F}\right)$ [22]. Chromium tends to increase the allotropic transformation temperature; therefore, the transformation temperature is somewhat higher in Stellite alloys [3]. This 
element also contributes as a solid-solution strengthener; significantly improves corrosion, oxidation and sulfidation resistances, and serves as the primary carbide former which impart wear resistance in Stellite alloys $[1,7]$. The main carbide in Stellite alloys is a chromium-rich $\mathrm{Cr}_{7} \mathrm{C}_{3}$ type which has a high melting point amongst the carbides present in the alloys. The chromium-rich $\mathrm{Cr}_{23} \mathrm{C}_{6}$ carbide is also common in low-carbon alloys such as Stellite alloy $21[1,29]$.

- Tungsten and Molybdenum: These elements are known as refractory metals with the melting temperatures of $3422^{\circ} \mathrm{C}\left(6192^{\circ} \mathrm{F}\right)$ and $2623^{\circ} \mathrm{C}\left(4753^{\circ} \mathrm{F}\right)$ respectively. The density of tungsten is recorded as $19.25 \mathrm{~g} / \mathrm{cc}\left(0.695 \mathrm{lb} / \mathrm{in}^{3}\right)$ while the density of molybdenum is $10.28 \mathrm{~g} / \mathrm{cc}\left(0.371 \mathrm{lb} / \mathrm{in}^{3}\right)$ [22]. Ranging from about 0 to $19 \mathrm{wt} \%$ in Stellite alloys, these elements participate as solid-solution strengtheners. When present as solute atoms, they provide additional strength to the cobalt matrix by impeding dislocation flow due to their large atomic size. If their content in the alloys exceeds about 5 atomic percent, $\mathrm{W}$ and Mo also contribute in carbide formation and promote the precipitation of $\mathrm{M}_{6} \mathrm{C}$. In addition, these elements serve to enhance the general corrosion resistance of the Stellite alloys $[1,6,11,17]$. It was reported that molybdenum addition in Stellite alloys (in preference to tungsten) imparts a greater degree of resistance to abrasive and adhesive wear. This is because Mo diffuses much more quickly since it has relatively smaller and much lighter atoms than $\mathrm{W}$. Therefore, under the same conditions, the volume fraction of carbides that imparts abrasive and adhesive resistance, in the Mo-containing Stellite alloys is more than that in the W- 
containing Stellite alloys $[7,29]$. Also, Mo imparts greater corrosion resistance than does $\mathrm{W}$ in a variety of wet corrosive media [1].

- Iron: Alloying addition of iron up to a maximum of $5 \%$ wt helps to stabilize the desired fcc matrix. This element has a density of $7.87 \mathrm{~g} / \mathrm{cc}\left(0.284 \mathrm{lb} / \mathrm{in}^{3}\right)$ and a melting point of $1538^{\circ} \mathrm{C}\left(2800^{\circ} \mathrm{F}\right)[6,22]$.

- Nickel: The metallic element nickel is commonly used in Stellite alloys as a solidsolution strengthener and the fcc matrix stabilizer. The common range of alloying addition of $\mathrm{Ni}$ in these alloys is between 0 and $4 \% \mathrm{wt}$ Nickel has a density of 8.90 $\mathrm{g} / \mathrm{cc}\left(0.249 \mathrm{lb} / \mathrm{in}^{3}\right)$ and a melting temperature of $1453^{\circ} \mathrm{C}\left(2651^{\circ} \mathrm{F}\right)[1,22]$.

\subsubsection{Machinability and Application}

The high toughness and hardness of Stellite alloys make them and their alloy parts very difficult to machine. Thus, Stellite products are preferred to be precisely cast so that minimal machining is required. Regarding the choice of machining; however, grinding is usually preferable to cutting. The machining tools for Stellite alloys are generally made of tungsten carbide and polycrystalline cubic boron nitride $[30,31]$.

The high-temperature applications of these materials include machine tools, cutlery, wear-resistant coatings, and structural components in aircraft and industrial turbines such as buckets and vanes [3]. In addition to the high-temperature usage, these alloys find widespread applications around room temperature. These applications include 
fracture fixation devices, orthopaedic prostheses for the shoulder, knee, and hip, dental implants, and dental crowns. Various in vitro and in vivo tests have shown that some Stellite alloys meet extremely high requirements with regard to mechanical, tribological and biocompatible properties including excellent resistance to degradation and outstanding corrosion resistance to body fluids [23,32]. Considering the volume fraction of carbides, the present-day Stellite alloys may be classified into three application areas as follows [1]:

- Low-carbon alloys $(<0.5 \mathrm{wt} \%)$ designed for high-temperature service

- Low to medium-carbon alloys $(0.5 \mathrm{wt} \% \sim 1.2 \mathrm{wt} \%)$ designed to combat only corrosion or simultaneous corrosion and wear

- High-carbon alloys $(1.2 \mathrm{wt} \% \sim 3 \mathrm{wt} \%)$ designed for wear service

\subsubsection{Tribocorrosion Properties}

The largest applications for Stellite alloys are for wear and corrosion resistant purposes. From a wear standpoint, Stellite alloys containing high levels of carbon (more than $1.2 \mathrm{wt} \%$ ) exhibit high hardness and are intended for use in applications where severe wear is involved. The several distinct types of wear generally fall into the following three main categories: abrasive wear, adhesive wear and erosive wear [1,33]. Abrasive wear occurs when hard particles like carbides or hard surface asperities are forced against or moved relative to a softer surface. The abrasive resistance of Stellite alloys which contain a hard phase generally increases by increasing the volume fraction of the hard constituent phase. Other factors significantly influencing the abrasion resistance include the shape 
and the size of the hard phase precipitates in the microstructure and the shape and the size of the abrading counterfaces $[1,29,34]$. Adhesive wear is often referred to the formation of localized bonding between the moving contacting asperities. Low coefficient of friction during sliding process and hard carbide particles in the matrix of Stellite alloys normally reduce their adhesive wear $[1,29]$. Erosive wear are caused by the impingement of small hard particles carried in a fluid stream against a surface. While ductility of Stellites can influence their erosive properties, the carbide volume fraction has very little effect on the erosion resistance of Stellite alloys $[1,35]$.

The single-phase alloys generally possess better corrosion resistance than multiphase alloys since the latter alloys have more interfaces between their phases. Although the high-carbon content Stellite alloys show some aqueous corrosion resistance, such resistance is limited because of the chemical segregation in their microstructures, shortage of chief alloying elements imparting corrosion resistance in their matrices, and the grain boundary carbide precipitation $[1,36,37]$. Thus, from a corrosion standpoint, Stellite alloys which contain low levels of carbon (less than $0.5 \mathrm{wt} \%$ ) are designed for severe corrosive environment applications. Other alloying elements such as $\mathrm{Cr}, \mathrm{Mo}$, and W are also added to Stellite alloys to enhance their resistance to corrosion.

The prominent examples for the three major groups of Stellite alloys include highcarbon alloys such as Stellite 1, 3 and 720, medium-carbon alloys like Stellite 6 and 694, and low carbon alloys including Stellite 21 and 25. Normally, the wear resistance 
increases with the higher volume fraction of carbides while corrosion resistance and ductility improve with lower carbon content.

The high-carbon Stellite 1 is a hardfacing alloy which possess excellent abrasion and average corrosion resistance for applications including pump sleeves, wear pads, rotary seal rings, expeller screws, and bearing sleeves. Stellite 1 has a high fraction of hard primary tungsten-rich $\mathrm{M}_{6} \mathrm{C}$ carbides, as shown in Fig. 2.2(a). This structure provides high hardness and exceptional wear resistance for Stellite 1. This alloy maintains its hardness at temperatures up to $760^{\circ} \mathrm{C}\left(1400^{\circ} \mathrm{F}\right)$. The main disadvantage of Stellite 1 includes its low toughness. Since this alloy is very hard and also more crack-sensitive compared to other Stellite alloys, it should only be finished by grinding. Also, like other high carbon Co-Cr-W-C alloys, Stellite 1 is inadequate for applications where harsh corrosive environments are involved [1,29].

The medium-carbon Stellite 6 alloy is one of the most widely used wear resistant cobalt based alloys due to its good all-round performance. Fig. 2.2(b) shows the typical hypo-eutectic microstructure of the cast form of Stellite 6 which contains the hard carbide phase dispersed in a tougher and more ductile $\mathrm{CoCr}$ alloy matrix. While the carbidic phase provides exceptional wear resistance for this alloy, its $\mathrm{CoCr}$ matrix imparts resistance to cavitation, galling, and corrosion. The major disadvantage of the cast form of Stellite 6 includes the presence of a semi-continuous brittle carbidic network in the alloy microstructure. The wrought form of this alloy, Stellite $6 \mathrm{~B}$, has a completely different structure. As shown in Fig. 2.2(c), the microstructure of Stellite 6B consists of 
broken up carbides which are dispersed evenly in the $\mathrm{CoCr}$ alloy matrix. During the manufacturing process, the semi-continuous network of carbides, which presents in the cast form of Stellite 6 , is broken up by the mechanical deformation experienced by Stellite 6B alloy. The resulting structure avoids the brittle carbidic network to act as the continuous crack-propagation sites in Stellite $6 \mathrm{~B}$ while maintains the hard carbides as the primary wear resistant component in the wrought alloy. Thus, the mechanical properties and toughness of Stellite $6 \mathrm{~B}$ are significantly superior to those of the cast version. Compared with the cast Stellite 6 , the wrought form exhibits dramatic improvements in the aqueous corrosion resistance because of its more uniform carbide structure. These properties along with the good machinability of Stellite $6 \mathrm{~B}$ have made the wrought alloy a good choice for critical applications where simultaneous wear and corrosion are involved $[1,38]$.

Stellite 21 is a low-carbon alloy which consists of a $\mathrm{Co}-\mathrm{Cr}-\mathrm{Mo}$ alloy matrix strengthened by dispersed hard carbides. Due to the low volume fraction of carbides, molybdenum is also employed in this alloy to strengthen the solid solution. The microstructure of Stellite 21 , which contains the eutectic $\mathrm{Cr}_{7} \mathrm{C}_{3}$ carbide and precipitate $\mathrm{Cr}_{23} \mathrm{C}_{6}$ carbide in the solid solution matrix, is shown in Fig. 2.2(d). Stellite 21 is considered as a corrosion resistant alloy which shows high corrosion resistivity to both reducing and oxidizing gaseous atmospheres up to $1150^{\circ} \mathrm{C}\left(2100^{\circ} \mathrm{F}\right)$. Compared with Stellite 1 and Stellite 6, Stellite 21 has higher corrosion resistance in reducing or complex environments. This is because of the fact that the main constituents of this alloy belong to the quatemary system of $\mathrm{Co}-\mathrm{Cr}-\mathrm{Mo}-\mathrm{C}$ rather than $\mathrm{Co}-\mathrm{Cr}-\mathrm{W}-\mathrm{C}$. In addition, due to the 
low carbon content in this alloy, most of the chromium is in the solid solution matrix instead of forming $\mathrm{M}_{7} \mathrm{C}_{3}$ carbides. Other than petrochemical and power generation applications, Stellite 21 is used on a large scale in medical applications including prosthetics, hip and denture implants because of its biocompatible properties $[1,39]$.

\subsubsection{Processing Methods}

Stellite alloys can be produced by various fabrication methods including casting, wrought, and $\mathrm{P} / \mathrm{M}$. The fabrication process of Stellite alloys affects their microstructures and their properties as explained before for Stellite 6B for instance. While each of these methods has its own advantages and disadvantages, the first two fabrication methods are used more often in industrial production of Stellites. This is mostly due to the fact that these two methods are highly adaptable to the requirements of mass production. Thus, large numbers of a given cast or wrought Stellite alloy may be produced very rapidly.

Despite their inherent advantages, the main disadvantage of the cast and wrought Stellite alloys is the problems associated with the chemical partitioning in these products $[37,40]$. Through the years, producers of these materials have increased the content of alloying elements to achieve different properties. For instance, the content level of chromium has been increased to obtain more corrosion resistivity. Also, more carbide forming elements such as molybdenum and tungsten have been used to improve properties such as wear resistance. Increasing alloying additions has led to the highly alloyed materials which are prone to microsegregation even under careful controls [11]. Thus, an undesirable microstructure with different types and morphologies of carbides as 
well as inhomogeneous distribution of alloying elements and carbides might be obtained in these alloys. As a result, Stellite alloys sometimes show anisotropic-like behavior due to their nonuniform structures. This ultimately leads to the lower yield strength, lack of toughness, lower wear resistance, and severe cracking in the final products $[6,41]$.

Another disadvantage of these two methods is their need for postproduction processing (e.g., grinding, heat treatment, thermomechanical processing, etc.) which increases the production cost $[5,11]$. One reason that Stellite alloys are normally expensive is because of their high machining cost due to their difficult machining process. Also, a full density Stellite alloy is hard to obtain using the common cast and wrought methods; thus, some properties such as corrosion resistance of these alloys would be affected due to the porous nature of the materials [5,32]. Many of these problems either cannot be found in the $\mathrm{P} / \mathrm{M}$ products of Stellite alloys or exist to a much lower extent. These advantages along with the near-net-shapemaking capability of the $\mathrm{P} / \mathrm{M}$ fabrication method have generated more interests in the fabrication of the $\mathrm{P} / \mathrm{M}$ Stellite alloys in the recent years. However, the start-up costs for acquiring the required equipments for any $\mathrm{P} / \mathrm{M}$ fabrication method remain as the main barrier in mass production of the $\mathrm{P} / \mathrm{M}$ products.

\subsection{Composite Materials}

\subsubsection{Types of Composite Materials}

Composites are macroscopic combinations of two or more distinct constituent materials between which a recognizable interface exist. The two categories of required 
constituent materials in a composite include matrix and reinforcement. The matrix is a continuous constituent that surrounds, binds together, supports, and provides form to the reinforcement constituent. The reinforcement constituent is an array of materials whose principal purpose is to provide superior level of structural properties such as strength and stiffness to the matrix. The reinforcing materials may also impart their special electrical, thermal, and tribological properties to enhance the matrix properties to achieve a particular balance of properties for a given range of applications [42].

Composite materials can be classified at two distinct levels. The first level of classification is usually made with respect to the reinforcing constituent which include particulate reinforced-, whisker reinforced-, fibre reinforced-, and woven fabric reinforced-composites. The second level of classification refers to the matrix constituent. This includes organic-matrix composites (OMCs), ceramic-matrix composites (CMCs), and metal-matrix composites (MMCs) [42].

\subsubsection{Metal Matrix Composites}

Like all composites, metal matrix composites consist of at least two chemically and physically distinct constituent materials; i.e., matrix and reinforcement, which are properly distributed to supply properties not obtainable with either of the individual materials. The matrix constituent is a completely continuous metal or metallic alloy such as aluminum, titanium, magnesium, beryllium, copper, silver, iron, nickel and cobalt. The lighter weight aluminum-, titanium-, and magnesium-based MMCs are most widely used for structural, automotive, aerospace, rail ground transportation, and recreational 
products. Beryllium-, copper-, and silver-based MMCs have applications in thermal management and electrical contacts. In high-temperature and wear-resistant applications such as rollers and tool dies iron-nickel, cobalt, and cobalt-nickel matrices are common $[42,43]$.

The reinforcing constituent, which is added extrinsically or formed internally by chemical reaction, is embedded into the matrix with a range from 1 to $70 \mathrm{vol} \%$ of the composite. The reinforcement materials, which might be metallic, ceramic, or organic compounds, are used to improve structural properties and/or enhance physical properties such as wear resistance, friction coefficient, or thermal conductivity [42]. These materials can be either in continuous or discontinuous form or a combination of both these forms. The term continuously-reinforced MMCs is commonly used to indicate metal matrix composites having reinforcements in the form of sheets, monofilament wires or continuously aligned long fibres. Since the reinforcements are embedded into the matrix in a certain direction, the result is an anisotropic structure in which the alignment of the reinforcing materials affects the directional properties of the composite [43].

Discontinuously reinforced MMCs use a reinforcing phase in the form of particles, platelets, whiskers, and short fibres. Compared with the continuously reinforced composites, discontinuously reinforced MMCs have relatively isotropic properties due to the presence of more randomly oriented reinforcements in the matrix. In addition, discontinuously reinforced MMCs are less expensive to make. Cost is an essential parameter, especially in large volume applications such as the automotive industry. 
Furthermore, conventional metallurgical processing techniques including liquid metal casting or powder metallurgy can be employed for discontinuously reinforced metal matrix composites, followed by common secondary processing techniques such as extrusion, forging or rolling [42-44].

As a diverse class of materials, metal-matrix composites have a wide variety of structural, wear, high temperature, electrical and thermal management applications. The properties of MMCs depend upon the characteristics of matrix constituent, reinforcing constituent, and the matrix-reinforcement interface. The advantageous properties of MMCs may be subdivided into two different parts.

- Advantages with respect to other composites: Compared with other types of composites - CMCs and OMCs - MMCs offer many distinct advantages which are mostly because of the contribution of the metal alloy matrix in these composites. These advantages include superior strength and stiffness, improved service temperatures, better electrical and thermal conductivities, higher radiation survivability, and enhanced joining characteristics. This type of composite is also nonflammable with little or no out-gassing or moisture absorption problems $[42,45]$.

- Advantages with respect to unreinforced metals: Due to the incorporation of the reinforcement, MMCs have superior mechanical properties compared with the metal matrix itself. The improved properties include hardness, strength, stiffness, 
creep and fatigue resistance. However, the improvements in these properties come at the expense of inferior ductility and fracture resistance of MMCs since the ductility and toughness of most reinforcements are very low. Also, the presence of appropriate reinforcing elements may significantly enhance the wear resistance of the base material. In addition, elevated temperature stability and highertemperature operating limits of MMCs are known to be superior to those of the unreinforced matrix materials. Moreover, metal-matrix composites are capable of providing better dimensional stability and controlling thermal expansion. Another valuable characteristic is the major weight saving of metal matrix composites because of the higher strength-to-weight ratio $[42,43,45]$.

\subsubsection{Carbon Fibre Reinforced MMCs}

Carbon fibre represents the best known reinforcement fibre in all kinds of advanced composites including MMCs. These materials have been used widely with the aluminum matrix to produce low density/high strength composites which are employed in primary structural applications and internal combustion engines for instance. However, in these composites the reaction of carbon with the matrix material at high temperatures during fabrication process or under service conditions (above $500^{\circ} \mathrm{C}$ ) generates a brittle compound of $\mathrm{Al}_{4} \mathrm{C}_{3}$ on the surface of the fibres that affects the strength and functionality of CF/Al composites at room or elevated temperatures [46-49]. Diwanji et al. [50] showed that up to $500^{\circ} \mathrm{C}$ the strength of CF/Al composites declined slightly, while it was considerably degraded for composites exposed to higher temperatures. 
The tribological properties of carbon fibre reinforced aluminum matrix composites were investigated by many researchers. Daoud [51] showed that the addition of CF remarkably improved the wear resistance of $2014 \mathrm{Al} / \mathrm{CF}$ over pure $2014 \mathrm{Al}$ alloy because of the formation of a lubricating film on the worn surface of the composites. Kong et al. [52] proved that this lubricating film composed of graphite and metal oxide. Song et al. [47] investigated the sliding friction and wear properties of $\mathrm{Al} / \mathrm{Al}_{2} \mathrm{O}_{3} / \mathrm{C}$ hybrid MMCs and concluded that their wear resistance was significantly improved over $\mathrm{Al} / \mathrm{Al}_{2} \mathrm{O}_{3}$ composites because of the reduced friction force between MMCs and their counterface material. Lee et al. [48] made the same conclusion and indicated that the unreinforced aluminum alloy suffered from a severe complex wear regime while the wear mechanisms in hybrid $\mathrm{Al} / \mathrm{Al}_{2} \mathrm{O}_{3} / \mathrm{C}$ composites included abrasive and slight adhesion. Liu et al. [46] concluded that increasing the volume fraction of short carbon fibres decreased the friction coefficient and wear mass loss of the CF reinforced aluminum matrix composites since short carbon fibres reduced direct contact between the matrix and its counterpart. Effects of fibre-orientation on friction and wear properties of short CF reinforced $\mathrm{Al}_{2} \mathrm{O}_{3}$ matrix composites were investigated by Jun et al. [53]. The wear properties of the normal-oriented composites were better than that of the parallel-oriented composites and the former composited had smoother worn surface with less microcracks than the latter composites.

Carbon fibres are also used to reinforce copper $(\mathrm{Cu})$ alloys. Xu et al. [54] investigated the wear behavior of $\mathrm{Cu} / \mathrm{CF}$ and demonstrated that the incorporation of carbon fibres notably improved the wear resistance of the reinforced composites compared with pure copper. Xia et al. [55] compared the tribomechanical properties of 
$\mathrm{Cu} / \mathrm{Zn} / \mathrm{Sn} / \mathrm{CF}$ composites and a common leaded copper alloy $(\mathrm{Cu} / \mathrm{Zn} / \mathrm{Sn})$. The results indicated that the composites possessed higher hardness, bending strength, wear resistance, and antifriction properties than the unreinforced copper alloy. Jun et al. [56] studied the effects of the short carbon fibre volume fraction on wear performance of the lead free tin bronze matrix composites. As the fibre volume fraction increased, the friction coefficient and the wear rate of the composites decreased.

Two predominant factors for the wide usage of CFs can be described as follow:

- First, despite the complex manufacturing technology of carbon fibres, it is more amenable to large-scale production compared with the production of other fibres. After 40 years of fabrication and use, CFs are now on the brink of broad commercialization. The utilization of carbon fibres has been growing rapidly because of their increasing availability and considerable price reduction from the 1990 s $[42,57]$.

- Second, carbon fibres have very useful engineering properties including good mechanical properties, high chemical inertness, excellent electrical and thermal conductivities, outstanding thermal stability, and low density; that can be readily translated into great flexibility in formulating composites with outstanding specific performance [57].

The raw material used to make carbon fibers is called the precursor. Depending on the precursor and the processing route, carbon fibres are available in a wide range of forms, diameters, and lengths; having even wider types with different mechanical 
properties such as strength and modulus. The several forms of carbon fibres can be divided into continuous fibres and short fibres, including chopped fibres and milled fibres $[43,57]$. Two major types of carbon fibres based on their precursors include polyacrylonitrile-based fibres (PAN fibres) and pitch-based fibres both of which have good chemical resistance to corrosive environments. PAN-based fibres, which are more commonly used, offer low to high modulus of about 240 to $310 \mathrm{GPa}\left(35\right.$ to $45 \times 10^{6} \mathrm{psi}$ ) and the highest strengths ranging from 3515 to $6380 \mathrm{MPa}$ (510 to $925 \mathrm{ksi}$ ). The increase in strength also translates into an increase in elongation-to-failure (up to $2.2 \%$ ) since carbon fibres exhibit linear stress-strain behavior to failure. Pitch-based fibres have low strength and high to ultra-high modulus ranging up to $825 \mathrm{GPa}\left(120 \times 10^{6} \mathrm{psi}\right)$, attractive for stiffness critical applications such as satellite structures $[26,42]$.

The process for making carbon fibers involves both chemical and mechanical operations. The majority of today carbon fibers are known to be PAN-based. Therefore, the typical sequences of operations to make these fibers are explained here. The process starts with spinning operations, which include polymerization, washing, and stretching the precursor into the desired fiber diameter. The next operation is stabilizing the fibers by oxidation process that involves heating the fibers in air in a range from $200{ }^{\circ} \mathrm{C}(392$ $\left.{ }^{\circ} \mathrm{F}\right)$ to $399^{\circ} \mathrm{C}\left(572^{\circ} \mathrm{F}\right)$. The process continues by carburizing the fibers in oxygen-free furnaces by heating the fibers in a temperature range between $700^{\circ} \mathrm{C}$ and $2480^{\circ} \mathrm{C}(1292$ ${ }^{\circ} \mathrm{F}$ and $4500^{\circ} \mathrm{F}$ ). The next step is surface treatment which involves oxidizing the surface of the fibers to improve their bonding properties. The last operation is sizing in which the fibers are coated to protect them from damage during handling or processing $[42,43,57]$. 


\section{Chapter 3: Specimen Fabrication}

\subsection{Raw Materials}

\subsubsection{Matrix Material}

The matrix material of the developed Stellite-alloy-based composites was prealloyed Stellite 25 powder, which is a special low carbon, tungsten-strengthened cobalt-chromium alloy, produced at Deloro Stellite Group. The main reason for selecting Stellite 25 is that the microstructure of this alloy has very small volume fraction of carbides (less than $4 \%$ ) due to its very low carbon content $(0.1 \mathrm{wt} \%)$. In this case, the effect of carbides in the alloy can be kept as low as possible. Stellite 25 is the cast version of the wrought alloy L605 with a nominal chemical composition presented in Table 3.1. The available forms of this alloy include castings and welding consumables and it can be formed by conventional techniques, including cold working, machining, forging, and welding. The alloy can be readily welded by both manual and automatic welding methods, such as Gas Metal Arc (GMAW), Shielded Metal Arc (coated electrodes), electron beam welding and resistance welding techniques.

The prealloyed powder form of Stellite 25 with a median particle size of $34 \mu \mathrm{m}$ was provided by Deloro Stellite Group for this research. This alloy combines exceptional thermal fatigue resistivity with good resistance to corrosion, oxidation and carburization 
up to $1038^{\circ} \mathrm{C}\left(1900^{\circ} \mathrm{F}\right)$ for prolonged exposures. The alloy is resistant to wet chlorine at ambient temperature and resists nitric and hydrochloric acids at certain concentrations and temperatures. As a non-magnetic material, this alloy has been found to possess good thermal cracking resistance and hot metal-on-metal wear resistance. However, it is not recommended for severe wear conditions and low stress or low angle particle erosion service. This is because the microstructure of this alloy is relatively free of carbide reinforcement due to the low carbon content in the alloy. The physical and mechanical properties of Stellite 25 alloy are presented in Tables 3.2 and 3.3 respectively. The combination of these properties makes this alloy suitable for a number of component applications in furnace hardware and metal working tools such as piercing points, forming tools, and extrusion dies $[1,58]$. According to its material safety data sheet (MSDS), Stellite 25 has few health hazards under normal handling and use. However, performing certain processes such as machining, grinding, welding on this material can produce dust, fumes, or particulate containing the component alloy elements [59]. Thus, proper ventilation is required when performing these processes to control the exposure to airborne toxic chemicals.

\subsubsection{Reinforcement Material}

In order to select the reinforcing agents properly, a wide range of possible additives and reinforcements including particles (0-D) like graphite and fibres (1-D) such as glass and carbon fibres was studied. Although it is impossible to theoretically predict the precise behavior of reinforcements in a metal matrix, the effort was to select the reinforcing elements with higher chances of success. Therefore, the method adopted to 
solve the problem was empirical and evolutionary, while guided by previous experience in the design of MMCs. The composites properties were estimated by the rules of mixtures. These are mathematical expressions which are used to approximate estimation of composite material properties in terms of the properties, quantities, and arrangement of their constituents. The usage of fibres was preferred to particles for two reasons. First, a fibre is inherently stronger than its bulk material since the flaw size in the fibre is limited by its small diameter. Second, if propagation of flaws in a fibre produces a failure in that fibre, this failure will not propagate to the other fibres to fail the entire composite as would normally happen in the bulk material [42]. Finally, carbon fibre was selected to be the reinforcing agent in the developed composites because of its large-scale production and functional engineering properties, including low density, high chemical inertness, excellent thermal stability, good mechanical properties and outstanding thermal conductivity, which are all discussed in Chapter 2.

Compared with glass fibre, carbon fibre has lower density and higher thermal conductivity. The thermal conductivity of carbon fibre filled composites was reported to be 10 to 15 times more than that of the corresponding glass fibre reinforced compounds [57]. In addition, carbon fibre is considered as a high-strength and high-modulus reinforcement material. Therefore, it was expected that the composites reinforced with carbon fibre would possess superior structural efficiency to the pure Stellite 25 matrix material because of their good specific strength and specific stiffness (material strength or stiffness divided by its density). To this end, two different types of PAN-based carbon fibres were selected for this research to be individually mixed with the Stellite 25 powder 
in order to make two groups of carbon fibre reinforced composites. Various properties of these two groups of composites such as their tribological, mechanical and corrosion properties were compared with each other and also with pure Stellite 25 alloy to optimize the final product.

The above fibres were obtained from Toho Tenax Company in chopped shape. This shape allows avoiding areas with inhomogenity in the final composites, i.e. isotropic properties in the composites due to the random orientation of reinforcements. Another reason for using the chopped shape of carbon fibres is that the fabrication techniques for discontinuously reinforced composites are usually more cost-effective than that of the continuous ones since the former composites can be processed using similar techniques to those used for their matrices [42].

The first product is a plain carbon fibre with $3 \mathrm{~mm}(1 / 8 \mathrm{in})$ length and $0.007 \mathrm{~mm}$ $\left(2.7 \times 10^{-4}\right.$ in $)$ diameter. This product provides good processability and good translation of mechanical and electrical properties [60]. The second type of carbon fibre is a nickelcoated chopped carbon fibre with $6 \mathrm{~mm}(1 / 4 \mathrm{in})$ length and $0.0075 \mathrm{~mm}\left(2.95 \times 10^{-4}\right.$ in) filament diameter. The thickness of nickel layer is about $0.25 \mu \mathrm{m}$ which corresponds to a weight fraction of $44 \% \mathrm{Ni}$. This product combines the excellent mechanical properties of carbon fibre with the properties of nickel such as high electrical conductivity. Applications of this type of carbon fibre include structural reinforcement, conductive composites for lightweight electromagnetic interference (EMI) shielding, radio frequency interference (RFI) shielding, and lightning strike protection for aircraft [60]. The nickel- 
coating on the carbon fibres serves three intended functions. One is to enhance the interface bonding between the carbon fibres and the matrix. The second is to prevent diffusion of carbon from the carbon fibres into the matrix, thus avoiding formation of carbides in the matrix. The third is to alleviate redox reactions including carbon oxidation and carbon reduction at elevated temperatures during manufacturing process or final service. The physical and mechanical properties of these fibres are presented in Table 3.4 to Table 3.7.

\subsection{Mixture of Powders}

\subsubsection{Chemical Composition of the Composites}

Pure Stellite 25 and eleven different homogeneous compositions of Stellite 25based composites, listed in Table 3.8, were prepared for specimen fabrication. In general, the final properties of fibre reinforced-composites can be controlled by selecting the reinforcing materials with desired properties, controlling the volume fraction of reinforcements, and specifying the fibre orientation. Therefore, the type of reinforcements, their content level and the fibre orientation were carefully investigated for the composites developed in this research. The specific objectives of using carbon fibres as reinforcement material in the composites are to improve their thermal conductivity, anti-friction properties, wear resistance, and mechanical properties. In addition, using the chopped form of carbon fibres can result in fabrication of randomly oriented fibre reinforced-composites which exhibit uniform properties in all orientations. As shown in Table 3.8, specimen A was used to measure the properties of pure Stellite 25 and also to investigate and optimize the fabrication parameters. Composites B, C, D, E 
and $F$ were used to study the role of chopped carbon fibre and its content level in the properties of the composites. Similarly, composites $K, L, M, N, O$ and $P$ were designed to investigate the effects of the chopped $\mathrm{Ni}$-coated carbon fibre and its content level on the composites.

\subsubsection{Powder Blending}

After preparing the compositions of the designed composites, blending process was performed using glass bottles, which were half filled with the constituent materials of the composites so that there was enough space for blending. Stellite 25 powder and carbon fibres were mechanically mixed for an hour in a three dimensional Turbula type T2C mixer, shown in Fig. 3.1. This apparatus mixes the materials by rotation, translation, and inversion. The mixing process took several cycles until enough powder mixture was produced for each composition of the composites.

\subsection{Fabrication Process}

\subsubsection{Hot Isostatic Pressing}

The technique used to fabricate composites has a significant effect on the final properties of the products [42]. In the current research, the hot isostatic pressing technique was employed to obtain the specimens. HIP is a powder metallurgy processing route to manufacture materials or components, which was invented at Battelle Material Institute in US in 1955 as a method to diffuse dissimilar materials. Diffusion bonding depends on atomic transport across two mating surfaces, including solid-to-solid, powder-to-solid, and powder-to-powder, and is adopted to remove bond line pores. These 
materials are bonded together by using a multiplier effect of high temperature and isostatic pressure [11].

The conventional HIPing process involves the following activities: producing spherical powders, screening powders to an acceptable maximum particle size, evaluating powders' cleanliness, blending the powders and reinforcements to obtain a homogenized distribution, loading the blended materials into containers, vacuum outgassing and sealing the containers, applying isostatic high-pressure gas to the containers, and consolidating the powders and reinforcements at an elevated temperature. For some applications, materials consolidated by HIP are consolidated again by further thermomechanical processing such as extrusion and forging to enhance mechanical properties, particularly high-temperature strength and ductility. In the consolidating step, the powders and/or reinforcements are plastically deformed that results in porosity elimination and full theoretical density achievement in the parts $[1,11,41]$.

\subsubsection{Preparation of Containers}

Containers for consolidation are commonly made from aluminum, mild steel, or stainless steel depending on the HIP parameters and the blended materials. The powderfilled containers can be designed and produced as simple geometric shapes or complex near-net shapes $[5,11]$. In the present work, round tubes of stainless steel-grade 316 having $40 \mathrm{~cm}(15.75$ in.) length and $1.5 \mathrm{~mm}(0.06 \mathrm{in}$.) wall thickness with inside diameters of $1.3 \mathrm{~cm}(0.51 \mathrm{in}$.) were used as consolidation containers for fabricating the 
specimens (Fig. 3.2). The use of the round tubes allows a uniform isostatic pressure to be applied to the blended materials and also avoids any areas of stress concentration.

The containers were filled with the blended materials and the tubes were sealed at one end. The mould filling plays an important role in the quality of the final products. To avoid contamination of the mixture, moulds were cleaned with acetone prior to each filling process. Care was taken to fill the moulds uniformly, since improper mould filling could cause uneven densities, cracks, or distortions in the final products. Therefore, a glass funnel was used in this step to uniformly pour the mixture into the moulds. Successive partial charges and tamping to gain the designed fill height were avoided since these could lead to the contamination problem, charge to charge interface and possible cracking at the interfaces along the height.

After loading the blended materials into a container, it is required to minimize the mixture adsorbed surface gases. This can be accomplished by subjecting the mixture to a vacuum and heating the mould to remove air and adsorbed moisture [5]. Therefore, the open end of each tube was connected to a vacuum hose and hot outgassed at $300^{\circ} \mathrm{C}(572$ ${ }^{\circ} \mathrm{F}$ ) for three days to a vacuum level of $10^{-5} \mathrm{mbar}$ using a vacuum pump, Fig. 3.3 ; then the tube was sealed and welded to eliminate the possibility of trapping air in the specimens.

\subsubsection{Differential Scanning Calorimetry}

Determination of the HIP/sintering elevated temperatures depends on a complex interaction of many factors including shape, size, and chemical composition of the 
composite. Therefore, these factors need to be considered when designing the HIP/sintering cycle. For instance, an excessively low HIP/sintering temperature may result in poor mechanical properties of the final parts [1]. As carbon fibres do not melt during the sintering process of the composite mixtures, since the melting point of carbon is about $3500{ }^{\circ} \mathrm{C}\left(6332^{\circ} \mathrm{F}\right)$, the solid state sintering method was intended to be used in this research. In this method, the sintering elevated temperature is below the melting point of the base material and usually between 50 and $90 \%$ of the melting point of the constituent with the lowest temperature in the system. Most of the commercial HIP activities with steel, nickel and cobalt alloys are typically performed between $1100^{\circ} \mathrm{C}$ $\left(2012^{\circ} \mathrm{F}\right)$ and $1205^{\circ} \mathrm{C}\left(2201^{\circ} \mathrm{F}\right)[5]$.

Differential scanning calorimetry is a thermal analysis technique which measures the difference in the heat flow rate to the sample and to a reference sample as a function of temperature while these are subjected to a controlled temperature program $[61,62]$. DSC thermograms can be used as the basis of designing sintering cycles in terms of the phase transformation temperatures and to evaluate the sample purity as a quality control instrument $[61,63]$. Another main application of DSC is in studying the phase formation and transformation temperatures, including melting, liquidus, solidus, and glass transition temperatures in materials [61].

The facility employed in this research was a Netzsch 404C Pegasus heat flux DSC shown in Fig. 3.4. In a heat flux DSC machine, the reference and the sample are placed in two similar crucibles. The crucibles are then placed in a single furnace on a metal disk to 
make a low resistance heat conduction path between the two holders. The furnace then undergoes a well-defined temperature program to perform the DSC analysis by detecting the difference in heat flow between the reference and sample throughout the test [61].

Before starting the DSC tests, the DSC machine was carefully calibrated and the baseline corrections were performed with empty sample and reference crucibles. The tests were carried out in an inert gas atmosphere by repeatedly evacuating and backfilling the furnace with argon gas. The reference material was alumina $\left(\mathrm{Al}_{2} \mathrm{O}_{3}\right)$ powder which did not experience any thermal events over the operating temperature range [64]. The sample and the reference material were precisely weighed and then were placed in the crucibles. To avoid reactions between the sample and crucible, alumina crucibles were used for the tests. Also, the sample was covered by alumina powder to improve the thermal contacts between the sample and the crucible.

Fig. 3.5 illustrates the heating/cooling cycle of the DSC tests performed in this research. The heating/cooling rates of the cycle were set to $20^{\circ} \mathrm{C} / \mathrm{min}\left(68^{\circ} \mathrm{F} / \mathrm{min}\right)$ while the initial standby temperature was $80^{\circ} \mathrm{C}\left(176^{\circ} \mathrm{F}\right)$. The maximum DSC heating temperature of $1500^{\circ} \mathrm{C}\left(2732^{\circ} \mathrm{F}\right)$ was selected based on the melting point of cobalt (1493 ${ }^{\circ} \mathrm{C}$ ). Both sample and reference crucibles were weighed again after each test to record any possible weight change. The DSC tests on Stellite 25 and Stellite 25 based composites were performed. To ensure accuracy of the tests, at least two specimens were tested for each material under the same condition. The weights of the sample and the reference materials before and after each test were almost constant. Fig. 3.6 presents the curves 
obtained from the DSC tests. As presented in Fig. 3.6(a), the starting and ending points of melting for specimen $\mathrm{A}$ are $1384{ }^{\circ} \mathrm{C}$ and $1431{ }^{\circ} \mathrm{C}$ respectively. These temperatures were close enough to the melting range between $1329^{\circ} \mathrm{C}$ and $1410^{\circ} \mathrm{C}$ for the pure Stellite 25 alloy, reported by the manufacturer (Table 3.2), so that the test accuracy and the sample purity were being confirmed. The maximum sintering temperature was then determined in terms of the DSC curves. Since solid state sintering method was adopted, the sintering elevated temperature should be below the lowest peak temperature of the first phase transformation among all the DSC curves. The results show that the peak temperature for the pure Stellite 25 specimen, plain carbon fibre reinforced composites and Ni-coated carbon fibre reinforced composites are respectively $1425^{\circ} \mathrm{C}\left(2597^{\circ} \mathrm{F}\right), 1250^{\circ} \mathrm{C}\left(2282^{\circ} \mathrm{F}\right)$ and $1424^{\circ} \mathrm{C}\left(2596^{\circ} \mathrm{F}\right)$; thus, the maximum sintering temperature was selected to be 1150 ${ }^{\circ} \mathrm{C}\left(2102^{\circ} \mathrm{F}\right)$.

\subsubsection{Design of HIPing/Sintering Cycle}

The HIP process was continued by subjecting the sealed containers to a high hydrostatic pressure at an elevated sintering temperature in a high pressure containment vessel. The pressure was transmitted to the blended materials through the flexible mould and the mass became uniformly compacted. The elastic compression of the mass involved aligning the particles into the best possible packing array by slipping and sliding followed by establishing and enlarging contact points between adjacent particles $[11,65]$. Simultaneously, the blended materials experienced the sintering cycle to develop structural integrity in the composites by particle coalescence and void elimination through diffusion bonding. 
While diffusion bonding is generally enhanced by increasing the hydrostatic pressure and temperature, these parameters should be selected with respect to other metallurgical and economic considerations. The optimum pressure is the pressure sufficient to close up all pores along the bond line and to close internal pores to produce a void-free material. However, the pressure has to be low enough to minimize internal stress and avoid plane slippage that may generate microcracks. Superalloys are normally hipped to full density under a pressure of 105 to $205 \mathrm{MPa}$ (15-30 ksi). An inert gas such as argon, helium, or nitrogen is used as the pressure medium in the process, so that the material does not chemically react with the pressurizing gas $[5,11]$. The holding period at the sintering elevated temperature needs to be kept to a minimum to lower the cost and to potentially avoid any deleterious effects from formation of brittle intermetallics, secondary recrystallization, and excessive grain growth $[1,11]$.

For this research, the HIP and sintering cycle parameters including heat-up rate, cooling rate, hold periods, and peak temperature were preliminarily determined according to the published data for the P/M cobalt-base superalloys $[1,5,8,11,23,32,65]$ and the DSC test results. This design was optimized by varying these parameters in the sintering process of the pure Stellite 25 specimen and then evaluating the performance of the specimens. Finally, it was decided that all the specimens be hot isostatic pressed at the temperature of $1150^{\circ} \mathrm{C}\left(2102^{\circ} \mathrm{F}\right)$ under a pressure of $150 \mathrm{MPa}(21.75 \mathrm{psi})$ of argon gas for about $2.5 \mathrm{~h}$. Using the inert argon gas could eliminate the chance of redox reactions including oxidation of carbon and nickel to produce carbon dioxide $\left(\mathrm{CO}_{2}\right)$ and nickel oxide $(\mathrm{NiO})$ or the carbon reduction by hydrogen to produce methane $\left(\mathrm{CH}_{4}\right)$. To avoid 
mechanical stresses induced by the possible volume change during the sintering cycle and hence possible cracking in the composites, both heating and cooling rates were kept low at $10^{\circ} \mathrm{C} / \mathrm{min}\left(50^{\circ} \mathrm{F} / \mathrm{min}\right)$. In addition, to eliminating the chance of excessive thermal gradients in the objects under sintering, two holding periods at $460^{\circ} \mathrm{C}\left(860^{\circ} \mathrm{F}\right)$ and 800 ${ }^{\circ} \mathrm{C}\left(1472^{\circ} \mathrm{F}\right)$ were introduced in the cycle. The sintering cycle and the final sintered/cut specimens are presented in Fig. 3.7 and Fig. 3.8 respectively. 


\section{Chapter 4: Material Characterization}

\subsection{Microstructural Analysis}

\subsubsection{Objectives}

Service properties of a composite are affected by its structural components and their properties. The fabricated specimens were first examined using an optical microscope and a SEM machine before any other material characterization tests. The EDX technique was also used to reveal the phases present in the specimens. The purpose of these examinations was structural analyses, which provided the following:

(1) Confirming whether the sintering process was properly designed to render a good microstructure for the specimens.

(2) Identifying the main phases present in the microstructures of the specimens.

In addition, one of the most important tasks of this research was to investigate if carbon fibres induced carbides in the Stellite 25 matrix. Therefore the analysis of carbide volume fraction was performed on the specimens using an image analysis software package, Clemex Vision Lite ${ }^{\mathrm{TM}}$, Longueuil, Canada. 


\subsubsection{Surface Preparation}

To perform microstructural analyses, the surfaces of each specimen were properly prepared in the following steps:

(1) Cutting the metallographic specimens from the main body of the HIPed materials using a Struers Secotom-10 cutting machine, shown in Fig.4.1, with an $\mathrm{Al}_{2} \mathrm{O}_{3}$ abrasive cut-off blade according to the ASTM Standard E 3 - 01 [66]. Care was taken to minimize excessive heating by following the usual precautions such as proper cooling conditions to avoid any microstructure alteration. To improve handling, protect the specimen edge, and provide a uniform format for automated preparation, specimens were mounted by encapsulating into a compression mounting compound. The compound was the phenolic plastic whose general properties are presented in Table 4.1. The hot mounting press of Struers Co. (laboPress-3), shown in Fig. 4.2, with adjustable pressing parameters such as heating temperature, heating time, cooling time, and force was used to provide heat $\left(150-180^{\circ} \mathrm{C}\right)$ and force $(0-50 \mathrm{MPa})$ for mounting.

(2) Grinding the surfaces of the specimens. The details of this operation, which was performed by a Buehler Ecomet-4 semiautomatic machine, shown in Fig. 4.3, are presented in Table 4.2. The specimen holder of the machine is a fixed frame in which the pressure is applied on the specimens via the central column of the holder. Therefore, it is necessary to place the specimens symmetrically in order to obtain flat surfaces after grinding operation. Grinding operation was required to reduce the surface damage caused by cutting and to achieve planar specimens due 
to possible shrinkage induced in the sintering process. To minimize possible damage such as the reinforcement pull out of the composites, it is required to carefully select the load and the speed of the grinding operation. The silicon carbide ( $\mathrm{SiC}$ ) abrasive paper (320 standard grit size) was used with a contact load of $27 \mathrm{~N}(6 \mathrm{Lb}$.) and a spindle speed of $250 \mathrm{rpm}$, according to the ASTM Standard E $1-01$ and Buehler materials preparation guide $[66,67]$. Water was used as the coolant to reduce the generated heat during grinding.

(3) Polishing the surfaces of the specimens. The purpose of the polishing operation was to produce a reflecting or bright mirrorlike surface. Polishing operation includes rough and final polishing steps. Rough polishing is performed to remove the damage produced during planar grinding. As described in Table 4.2, specimens were roughly polished with sequentially decreasing abrasive paper grit sizes. Final polishing was performed to eliminate any surface damage. In the present research, specimens were lightly polished with $1 \mu \mathrm{m}$ diamond suspension and $0.05 \mu \mathrm{m}$ alumina suspension on two specific polishing pads. The specimens were thoroughly cleaned in an ultrasonic bath, prior to and after each step of the final polishing since surface quality could be degraded by abrasion from the debris produced during polishing.

(4) Etching the specimen surfaces. After polishing, the specimen surfaces were subjected to immersion and electrolytic etching with two different solutions. Because all constituent materials of the composites are corrosion resistant, 
vigorous etching reagents were necessary to optically enhance microstructural features. The first reagent was a mixture of $15 \mathrm{~mL}$ nitric acid $\left(\mathrm{HNO}_{3}\right)+15 \mathrm{~mL}$ acetic acid $\left(\mathrm{CH}_{3} \mathrm{COOH}\right)+60 \mathrm{~mL}$ hydrochloric acid $(\mathrm{HCl})+15 \mathrm{Ml}$ water $\left(\mathrm{H}_{2} \mathrm{O}\right)$, which is the standard solution for immersion etching of cobalt-based alloys. This solution must be aged for $1 \mathrm{~h}$ before use and the samples need to be immersed in the reagent for up to $30 \mathrm{~s}[1,68]$. The second reagent composition and also its etching procedures are presented in Table 4.3. These solutions and also etching procedures were selected because they are well known for identifying carbides in cobalt-based superalloys $[1,68,69]$.

\subsubsection{Apparatus}

The images of microstructural features of the specimens were obtained with an Olympus PM-63 light microscope, shown in Fig. 4.4. The microscope is capable of obtaining images in the magnification range of $50 \times$ to $1000 x$.

The scanning electron microscope is one of the most versatile instruments for investigating the microstructure of materials. An SEM has a greater resolution than a traditional optical microscope since it uses electrons instead of visible light that is used in optical microscopes. Also, because a SEM has a better depth of field than an optical microscope, the sharpness of the SEM images is much higher than that of the optical images. In the present research, a Hitachi S-570 model of SEM, shown in Fig. 4.5, with energy dispersive $\mathrm{X}$-ray spectrum was used to produce the high-resolution images of the specimens' microstructures and to analyze the chemical characterization of the 
specimens. The microscope is capable of obtaining images in the magnification range of $35 \times$ to $15000 \times$ and uses a tungsten filament thermionic emission gun as its electron beam emission source. Once cleaned and dried, the specimens were coated prior to mounting by a thin layer of graphite to become electrically conductive. This task was to prevent the accumulation of static electric fields on the specimen due to the electron irradiation required during imaging and also to improve the contrast of the image.

\subsubsection{Carbide Volume Fraction Analysis}

To identify carbides and thereby to determine the volume fraction of carbides in the specimens, image analysis software, Clemex Vision Lite ${ }^{\mathrm{TM}}$ was employed. To obtain accurate measurements, instrument calibration was made for each objective lens since the width represented by a pixel would change with magnification. Then the images obtained from the light microscopy of specimens subjected to the aforementioned first etching solution were loaded and the pictures went through different grey level operations to alter the intensity values associated with each pixel in order to polish up the grey images and produce high contrast pictures. The next step was thresholding to classify the pixels that fell within a restricted range of grey levels into an editable entity called a bitplane. These bitplanes were the objects measured finally rather than the underlying image. To remove the artifacts within one bitplane and eliminate the very small features, which should have not been inserted in a specific bitplane, several binary operations such as trap and chord size operations were performed. At this stage, the area percent of all detected phases was measured with respect to the total area covered by the field of analysis for each picture. The area with carbides was used to calculate the carbide volume fraction based on the 
Delesse principle, i.e., the ratio of the area occupied by a non-classically shaped object relative to the entire profile area is a consistent estimate of its volume fraction [6]. For each specimen, fifteen different images were captured and analyzed in three magnifications $(50 \times, 100 \times$, and $200 \times)$, respectively.

\subsection{Density Measurement}

Composite materials cannot usually achieve full density since in their microstructure porosity is inevitably present that undoubtedly affect the properties of the materials. However, the HIP/sintering powder metallurgy method normally results in porosity elimination in composites $[1,11,41]$. Therefore, a density measurement may be necessary for composite materials to determine if the part has been densified, either overall or in a critical region, to the degree required for the intended application.

The immersion density technique was employed in accordance with the ASTM Standard B 311-08 [12] to determine the density of the specimens. The density of water at room temperature was assumed to be $1 \mathrm{~g} / \mathrm{cm}^{3}$. This technique is based on Archimedes principles described below:

- Weigh the specimen in $\operatorname{air}\left(w_{d}\right)$

- Weigh the specimen in distilled water $\left(w_{f l}\right)$ while suspended by thin wires

The following formula was used to calculate the density of the materials $[12,70]$ : 


$$
\rho_{k}=\frac{w_{d} \cdot \rho_{n}}{w_{d}-\left(w_{n}-w_{\text {wires }}-w_{\text {bea ked }}\right)}
$$

where $\rho_{k}$ is the specific density of the specimen, $\mathrm{g} / \mathrm{cm}^{3}, W_{d}$ is the mass of the specimen in air, $\mathrm{g}, \rho_{f}$ is the density of distilled water, $\mathrm{g} / \mathrm{cm}^{3}, W_{f}$ is the weight of the specimen in distilled water, $\mathrm{g}, W_{\text {wires }}$ is the weight of the Kevlar wires, $\mathrm{g}$, and $W_{\text {beaker }}$ is the weight of beaker, $\mathrm{g}$.

The basic experimental apparatus, shown in Fig. 4.6, is a Sartorius 6080 electronic balance, which is accurate to $0.1 \mathrm{mg}$ and allows for both dry (at air) and wet (in bath) weighing of specimens. The setup includes a small metallic stand that fits into the balance and a small perforated plastic beaker that is suspended from the stand with two Kevlar wires. The beaker is submerged in a one-litre distilled water bath, allowing the specimen to be totally immersed while being measured. Care should be taken to avoid any contact between the beaker and wall of the water bath.

\subsection{Corrosion Test}

One of the main features of superalloys or Stellite alloys is their good corrosion resistance. However, these materials can corrode in several different ways. These alloys can be attacked by various corrosive aqueous media such as mineral or organic acids, oxidizing environment at ordinary or high temperatures, and different types of salts in the air necessary for combustion for instance. Also, hot corrosion might occur in gas turbines, incinerators, and boiler tubes due to the use of sulfur- and sodium-containing fuels $[1,11]$. To evaluate the corrosion resistance of the fabricated materials, immersion 
corrosion tests were performed on the fabricated specimens in accordance with the ASTM Standard G 31-72 [14]. This test was intended to represent a worst-case situation as a guide rather than a complete standardization by reproducing a certain environment or a specific condition of exposure. Therefore, the selected test medium was the oxidizing acid $65 \% \mathrm{HNO}_{3}$ at boiling temperature.

Since corrosion is a surface phenomenon, the surface condition of the specimen directly affects the results of the tests [71]. According to the ASTM Standard G 1-03 [13] the surface of the cylindrical specimens with $0.64 \mathrm{~cm}(0.25 \mathrm{in})$ diameter and $1 \mathrm{~cm}(0.4 \mathrm{in})$ height, Fig. 4.7, were polished to $0.05 \mu \mathrm{m}$ finish, cleaned in ultrasonic bath, and air-dried. The geometric area of each specimen was calculated to $1 \%$ accuracy and weighing of the specimens was accurate to $0.1 \mathrm{mg}$. The specimens were then immersed in the corrosive solution for 72 hours and were removed afterwards for examination. Shortly after removing from the medium, the specimens were properly cleaned by soft brushing under warm tap water to remove bulky deposits and corrosion products without significant removal of the base materials. Then the specimens were dried with ethanol and a blow dryer.

The mass loss of each specimen due to corrosion was determined by weighing the specimen before and after the corrosion test. To assess the corrosion damage, the average corrosion rates of the specimens were obtained as follows $[13,14]$ :

$$
\text { Corrosion Rate }=(B \times M) /(A \times t \times \rho) \text {, }
$$


where $B$ is a constant equal to $3.45 \times 10^{6}$ for desired corrosion rates in mils per year, $t$ is the time of exposure in hour, $A$ is the area of exposure in $\mathrm{cm}^{2}, M$ is mass loss in $\mathrm{g}$, and $\rho$ is the density of specimen material in $\mathrm{g} / \mathrm{cm}^{3}$.

\subsection{Hardness Test}

\subsubsection{Macrohardness}

Hardness represents the resistance of a material to permanent deformation or indentation [72]. It is generally known that hardness of a material relates closely to its resistance to wear. The harder the material, the more wear resistant it is [1]. In order to correlate the wear resistance with the hardness of the fabricated materials, the hardness of the fabricated specimens was determined on a Rockwell hardness tester in accordance with the ASTM Standard E 18-08b [15] in the standard laboratory atmosphere. Rockwell is considered as an empirical indentation hardness test that might be useful in selection of materials and in quality control [73]. The test determines the hardness of a material by using diamond spheroconical, tungsten carbide, or steel ball indenters to make an indentation on the surface of the testing specimen under specified conditions. For each measurement, the hardness is obtained by measuring the difference in depth of penetration as the force on the indenter is increased from a specified preliminary test force (preload) to a specified total test force (major load) and then returns to the preload. The test results, which are dimensionless numbers, may also correlate to some other characteristics of the specimens such as tensile strength, wear resistance, and ductility. There are different scales in Rockwell hardness test; therefore, the test results should be 
quoted as a number followed by the symbol HRX where $\mathrm{X}$ represents the scale designation $[15,73]$.

The test apparatus used in this research is a Wilson ${ }^{\circledR}$, Norwood, USA, Series 2000 Rockwell machine shown in Fig. 4.8. In this machine the depth measurement is automatically converted to the Rockwell hardness value. The hardness value and Rockwell scale are then indicated by an electronic device. The test specimens, $10 \mathrm{~mm}$ (0.39 in.) in thickness, were ground and polished to get smooth and flat surfaces at the top and on the bottom. The tests were conducted using a diamond spheroconical indenter with the preliminary test force of $10 \mathrm{kgf}(98 \mathrm{~N})$ and the total test force of $150 \mathrm{kgf}(1471$ N) as the test parameters. For each designation, at least ten different hardness measurements were performed and the average of hardness values was reported as the final hardness value of the designation.

\subsubsection{Microhardness}

A Microhardness Tester Unit, Model SMT-X7 Dual Indenter, shown in Fig. 4.9, with a load capacity of $1 \mathrm{kgf}(9.8 \mathrm{~N})$, was also employed in this research. This instrument allows measuring hardness in Vickers of a material on micro level, which means that hardness of each phase in the microstructure can be quantified. In order to measure the variations of hardness in the region around carbon fibres of the fabricated composites, a series of indentations were performed from the interface of carbon fibres and the alloy matrix gradually away to the matrix. The purpose of this test was to further confirm if any carbon diffusion had occurred in the fabricated composites. The carbon diffusion 
from carbon fibres into the alloy matrix would form carbides in the region around carbon fibres that cause alteration of the matrix hardness in the fibre-matrix interface region.

\subsection{Wear and Friction Tests}

\subsubsection{Sliding Wear}

A very important feature of superalloys and Stellite alloys in particular is their wear-resistance properties. One main intended application of the fabricated materials in this research is for wear resistance. Therefore, the tribological behavior of the fabricated materials was investigated under sliding wear using a TEER-POD-2, Droitwich, UK, computer controlled pin-on-disk tribometer, shown in Fig. 4.10.

Sliding or abrasive wear refers to a type of wear generated by the sliding of one solid hard surface along another softer surface [74]. Therefore, in a pin-on-disk test, two specimens are required which are known as the pin and disk. The pin used in this research was a $5 \mathrm{~mm}$ diameter AISI 52100 steel ball, provided by TEER Coating Limited, with the hardness of $60-67 \mathrm{HRC}$, equivalent to $697-900$ Vickers. The pin specimen was positioned perpendicular to a flat surface known as disk. The disk was the tested specimens cut from the HIPed materials with $10 \mathrm{~mm}$ thickness. These specimens were polished to $0.05 \mu \mathrm{m}$ finish, cleaned in an ultrasonic bath, and then dried. The masses of the polished, cleaned, and dried specimens were measured to $10^{-4} \mathrm{~g}$ accuracy before the wear test. 


\subsubsection{Test Parameters}

The tests were conducted under a dry-lubricating condition and in accordance with ASTM Standard G 99-05 [16]. The test parameters were selected as:

- Load: $10 \mathrm{~N}$, the value of normal force at the wearing contact.

- Speed: $0.1 \mathrm{~m} / \mathrm{s}$ selected as the relative sliding speed between the contacting surfaces.

- Time: $83 \mathrm{~min}$, the duration of each test.

- Distance: $\sim 500 \mathrm{~m}$, the accumulated sliding distance within the period of wear test, calculated from the time and the speed.

- Atmosphere: The tests were conducted in the standard laboratory atmosphere with the relative humidity of $40 \%$ and the temperature of $23^{\circ} \mathrm{C}$.

During the test the pin (ball) was wearing the disk (specimen) surface which was spinning at a rotational speed of $308 \mathrm{rpm}$ under a compressive force of $10 \mathrm{~N}$ for 83 min without lubrication. As a result, a wear track was formed in the specimen surface.

\subsubsection{Data Collection}

Three types of data were obtained from the pin-on-disk tests:

- Wear loss: After the test, the disk specimens were cleaned by soft-brushing under warm tap water to remove debris generated during the wear test, dried, and weighed to $0.0001 \mathrm{~g}$ accuracy. Assuming that there is no significant pin wear, the 
following equation was used to calculate the volume losses of the specimens due to the wear [16]:

$$
\Delta V=\frac{m}{\rho} \times 1000
$$

where $\Delta V$ is volume loss, $\mathrm{mm}^{3}, m$ is mass loss, $\mathrm{g}$, and $\rho$ is the density of the specimen, $\mathrm{g} / \mathrm{cm}^{3}$

- Wear factor: Using the formula expressed below, the wear factor or the specific wear rate of each specimen was obtained [16]:

$$
k=\frac{\Delta V}{F \cdot \Delta s},
$$

where $k$ is wear factor, $\mathrm{mm}^{3} / \mathrm{N} . \mathrm{m}, \Delta V$ is volume loss, $\mathrm{mm}^{3}, F$ is normal force, $\mathrm{N}$, and $\Delta s$ is sliding distance, $\mathrm{m}$.

- Friction coefficient: The coefficient of friction of each specimen was recorded automatically throughout the test with the aid of a linear variable displacement transducer so that the variation of the friction coefficient with time could be obtained.

To understand the wear mechanism of the fabricated composites and investigate the enhancement effect of the reinforcements on the friction and wear behavior of the 
composites, the worn surfaces of all the specimens were examined using optical microscopy and SEM along with the EDX spectroscopy for elemental analysis.

\subsection{Thermal Conductivity}

\subsubsection{Background}

Friction heat is generated at the rubbing surfaces of materials during wear. The produced heat causes adhesion of the contact surfaces and thereby speeds up the wear loss of the materials. In addition to their excellent wear resistance, carbon fibres possess good thermal conductivity, which helps dissipate the friction heat when they are added to the materials exposed to wear conditions. The reduction of friction heat would mitigate adhesive wear damage and thus enhances the overall wear resistance of materials. In order to confirm the beneficial effect of carbon fibres on friction heat dissipation, thermal conductivity of the fabricated specimens was investigated using a $240 \times 320$ pixel-IR camera, shown in Fig. 4.11, which has a thermal sensitivity of $20 \mathrm{mK}$ at $303 \mathrm{~K}$ and a spectral response in the long wave infrared from 8.0 to $8.8 \mathrm{~mm}$.

\subsubsection{Methodology}

Thermal conductivity, $K$, is defined as the intrinsic property of a material in heat transfer. When a temperature gradient exists in a material, heat conduction occurs by transferring energy through vibration between the material's adjacent atoms and molecules. Higher rates of heat transfer occur across the materials with high thermal conductivities than across materials with low thermal conductivities $[4,75]$. The variation of thermal conductivity between different materials can be analyzed using the pulsed 
thermography method. This method is known as an active thermographic inspection in which energy sources (flash lamps) are required to apply energy to the specimen of interest using a pulsed excitation in order to produce a thermal contrast between the specimen's front and back surfaces. During the cool down process, the temperature at the specimen's back surface can be detected by an infrared camera.

In the $240 \times 320$-pixel IR camera, two xenon flash lamps, each powered by a $2400 \mathrm{~J}$ power supply, were used as energy sources. Each sample was inspected from both the front and back surfaces. Each thermogram contained 740 frames that were acquired at $60 \mathrm{~Hz}$ and the last frame in the acquisition was obtained at $8.15 \mathrm{~s}[75]$.

The following formulas were used to calculate the thermal conductivity of the materials [75]:

$$
\begin{aligned}
& \Delta T=\frac{Q}{2 e \sqrt{\pi t}}, \\
& C K=\frac{e^{2}}{\rho},
\end{aligned}
$$

where $\Delta T$ is the temperature difference between the front and back surfaces, $\mathrm{K}, Q$ is the energy density applied at the surface, $\mathrm{J} / \mathrm{m}^{2}, t$ is the frame acquisition time, $\mathrm{s}, e$ is the thermal effusivity, $\mathrm{J} \mathrm{m}^{-2} \cdot \mathrm{K}^{-1} \cdot \mathrm{s}^{-1 / 2}, \rho$ is the density, $\mathrm{g} / \mathrm{cm}^{3}, C$ is the specific heat capacity, $\mathrm{J}$, and $K$ is the thermal conductivity, $\mathrm{W} \cdot \mathrm{m}^{-1} \cdot \mathrm{k}^{-1}$. From pulsed thermography experiments on an aluminum plate (A1 2024-T3) and the theoretical $e$ value calculated from literature [75], $Q$ was estimated to be $\sim 50,000 \mathrm{~J} / \mathrm{m}^{2}$. 


\section{Chapter 5: Experimental Results}

\subsection{Plain Carbon Fibre Reinforced Composites}

\subsubsection{Microstructure}

To verify the fabrication process parameters, the pure Stellite 25 specimen (specimen A) was examined by optical microscopy in an unetched condition, as shown in Fig. 5.1(a). The microstructure of this specimen looks well alloyed and dense with no obvious porosity and cracking. This confirmed that the processing parameters were properly selected. Fig. 5.1(b) shows the typical unetched microstructure of the composite specimens (specimen F) with the carbon fibres embedded in the Stellite 25 matrix. By closely examining the microstructures of the composite specimens, a few small voids can be found, as shown in Fig. 5.1 (c) for specimen C. The presence of voids is common in composites because of the incorporation of reinforcing materials that have different properties from the matrix material [65]. Also, the sintering process normally causes some voids in the matrix of composite materials because of the point contacts between the necked particles [65]. However, due to the low amount of porosity in the composites' microstructures, it can be inferred that the fabrication parameters were selected properly.

To identify the phases present in the microstructure of the fabricated specimens, they were first subjected to the etching procedure given in Table 4.3 and then examined 
by scanning electron microscopy. It was observed that the matrices of the composite specimens are similar to that of the pure Stellite 25 specimen. Fig. 5.2 shows the SEM micrograph of the matrix of the etched composite $\mathrm{F}$ in an area close to carbon fibres. The phases present in the matrices of the fabricated specimens include a primary Co solid solution matrix, an intermetallic phase, and two types of carbides: W-rich carbide in white and Cr-rich carbide in black. Due to the large content of W in Stellite 25 alloy, the amount of W-rich carbide $\left(\mathrm{W}_{6} \mathrm{C}\right)$ is much more than that of $\mathrm{Cr}$-rich carbide $\left(\mathrm{Cr}_{7} \mathrm{C}_{3}\right.$ and/or $\mathrm{Cr}_{23} \mathrm{C}_{6}$ ). Thus, the main carbide in the matrices of the fabricated specimens is the tungsten-rich carbide $\mathrm{W}_{6} \mathrm{C}$. The existing phases in the matrices of the fabricated specimens were further examined using the EDX technique. Fig. 5.3 shows the SEM image of the specimen $\mathrm{F}$ at high magnification together with the EDX spectra for phase identification. Firstly, the light grey area in Fig. 5.3(a) represents the fcc Co solid solution. Secondly, with the EDX major pick of W, the white phase is the tungsten-rich carbide. Thus, tungsten not only serves as a solid solution strengthener, but owing to the high content, also forms $\mathrm{W}_{6} \mathrm{C}$ in the matrix alloy. Thirdly, the dark grey phase in Fig. 5.3(a) represents the intermetallic phase containing $\mathrm{Co}, \mathrm{W}$ and $\mathrm{Cr}$, as identified in the EDX spectrum in Fig. 5.3(c). Finally, the black phase in Fig. 5.3(a) represents the Cr-rich carbide with the major picks of $\mathrm{Cr}$ and $\mathrm{C}$ as seen in its EDX spectrum in Fig. 5.3(d).

One main interest of this research is to investigate whether carbon fibres induce carbides in the composite specimens during the $\mathrm{P} / \mathrm{M}$ process. In order to measure the volume fraction of carbides in each specimen, the specimens were etched with the standard cobalt-based alloys' etching solution mentioned before in Chapter 4. The optical 
microstructure of the pure Stellite 25 specimen in the etched condition is shown in Fig. 5.4. The carbides (W-rich and Cr-rich) are identified in black in the cobalt-solid solution matrix after etching with this solution. After etching, the specimens were analyzed utilizing an optical microscope and the image analysis software Clemex Vision Lite ${ }^{\mathrm{TM}}$. The microstructures of all the specimens and some of the pictures used for the evaluation of carbide volume fraction are presented in Fig. 5.5, which shows the matrices of the developed materials in the vicinity of carbon fibres. In this carbide volume fraction analysis, the small carbides were neglected and only the large-size carbides were taken into consideration.

The corresponding volume fractions of carbides in each specimen are reported in Table 5.1. It is demonstrated that the carbide volume fractions in the composite specimens are slightly more than that in the pure Stellite 25 specimen. Also, the comparison in carbide volume fraction between the composite specimens suggests that the volume fraction of carbides increases with increasing the carbon fibre content in the specimens. These two observations indicate that the diffusion of carbon from the carbon fibres occurred at the high temperatures during the sintering process. However, the carbide volume fraction increment is very small since the maximum carbide volume fraction in specimen $\mathrm{F}$ (with $5 \mathrm{wt} \%$ of carbon fibre) is only 6.4 vol.\%. It is worth comparing the carbide volume fraction between Stellite alloys and the carbon fibre reinforced Stellite alloy composites. The volume fraction of carbides in the former varies between $3 \% \sim 50 \%$ while the carbon content varies between 0.1 to $3.3 \mathrm{wt} \%[1,22,76]$. However, the volume fraction of carbides in the latter varies only between $3.8 \% \sim 6.4 \%$ 
while the carbon content varies between $0.1 \mathrm{wt} \%$ in specimen $\mathrm{A}$ to about $5.1 \mathrm{wt} \%$ in specimen F (0.1 wt\% carbon in Stellite 25 plus $5 \mathrm{wt} \%$ carbon fibre). This implies that employing carbon fibre in Stellite alloys is superior to adding carbon, as viewed from the volume fraction of carbides in the alloys. Therefore, it is reasonable to conclude that the carbon diffusion from the carbon fibres during the sintering process does not significantly affect the volume fraction of carbides in the matrices of the composites.

\subsubsection{Density}

The results of the density test are presented in Table 5.2. The density of specimen $A$ is about $18 \%$ higher than the reported value for Stellite 25 alloy in literature [58]. This implies that the HIP densification process employed in this research for fabricating the Stellite 25 specimen was well designed and the Stellite 25 powder was densified with a combination of high pressure and high temperature applied in the consolidation process. This process led to a dense microstructure with very low porosity, which can be seen in Fig. 5.1(a). The pure alloy specimen has higher specific density than the composite specimens. This is because carbon fibre is a lightweight material with a lower density compared with Stellite 25 alloy. In addition, as mentioned before, the fabricated composites would technically have more voids compared with the matrix material due to the presence of fibres. As a result, the density of specimen $F$ that has the highest carbon fibre content is about $14 \%$ less than that of specimen $\mathrm{A}$.

\subsubsection{Corrosion Resistance}

The corrosion rates of the specimens, represented in mils per year, are shown in 
Fig. 5.6. It is demonstrated that the corrosion rate of Stellite 25 increases when reinforced with carbon fibres and the corrosion rate of the Stellite 25 composite increases with the carbon fibre content as well. It should be noted that the low-carbon Stellite alloys, Stellite 25 for instance, are mainly designed to combat corrosion while some other properties of these alloys such as wear resistance are sacrificed to some degree. However, the corrosion resistance of the fabricated composites is still quite high compared with highand medium-carbon Stellite alloys. Even specimen F, which has the lowest corrosion resistance among all the specimens, exhibits higher corrosion resistance than the medium-carbon $\mathrm{Co}-\mathrm{Cr}-\mathrm{W}$ alloy, Stellite 6B [76].

Under a similar condition of corrosion exposure, Stellite 6B (containing 1 wt\% carbon) showed a corrosion rate greater than 985 mils/yr [76], whereas composite F (containing $5 \mathrm{wt} \%$ carbon fibre) exhibited a corrosion rate of only about $192 \mathrm{mils} / \mathrm{yr}$, one fifth lower than that of Stellite 6B. It is well known that the corrosion resistance of Stellite alloys decreases with the increased carbon content because the presence of carbides initiates the interfaces in the alloys, which tends to aggravate the corrosion of the alloys $[1,76]$. In addition, Stellite alloys with more carbon content suffer more from the chemical segregation in their matrices and lack of chief alloying elements imparting corrosion resistance in their microstructures $[1,36,77]$. The comparison indicates that the fabricated composites would be more corrosion resistant than medium-carbon Stellite alloys. This result is attractive since it implies that with a same amount of carbon content, the alloy containing carbon fibre is much more corrosion resistant than the alloy containing carbon element. Therefore, it can be concluded that carbon fibre is superior to 
carbides in Stellite alloys with respect to corrosion resistance.

It should be emphasized that the highly corrosive environment simulated in this research is not aimed at duplicating a practical condition of exposure, but is intended to show the high corrosion resistivity of the fabricated composites. The reason lies in the fact that corrosion is a complex process that is simultaneously affected by the chemical composition of the exposed material and the environment of the exposure.

\subsubsection{Hardness}

About ten readings of hardness at different positions on each specimen were taken and the average of these measurements was reported as the Rockwell hardness of the specimen. As presented in Fig. 5.7, pure Stellite 25 (specimen A), has the lowest hardness among the specimens. Compared with the hardness value of cast Stellite 25 alloy, provided by the manufacturer in Table 3.3 , specimen A which was fabricated by the $\mathrm{P} / \mathrm{M}$ route shows about $41 \%$ increase in hardness. This increase was owing to the use of HIP process as the fabrication method, which naturally results in a material with lower porosity and higher density [11]. The hardness of specimen A increases with addition of carbon fibres. It can be observed that the hardness of Stellite 25 can be enhanced up to $49 \%$ by incorporating $5 \mathrm{wt} \%$ carbon fibre (specimen F). This improvement in the hardness is due to the fact that these fibres are inherently harder than the matrix material. The hardness values of Stellite 25 based composites can also be influenced by the presence of slightly increased carbides due to diffusion of carbon fibres in these materials as well. 


\subsubsection{Friction and Wear Resistance}

The evolution of the friction coefficients of the test materials during the wear test are presented in Fig. 5.8. As illustrated, the friction coefficients for each specimen go up at the initial stage until reaching a narrow peak. The coefficients of friction then drop to lower values and become more or less constant afterwards. While the constant values of friction coefficient for all the composite specimens vary in a range of $0.13<\mu<0.16$ after about 35 min sliding, the coefficient of friction for the pure Stellite specimen is 0.31 after 55 min sliding. The significant reduction in friction coefficients of the composites can be attributed to the generation of a thin transfer film of graphite from the carbon fibres on the counter surfaces. This behavior is in agreement with the analyses carried out by other researchers $[42,46,47,48,51]$. Under the cyclic load occurring during the wear process, the surface of the specimen experienced plastic deformation [78]. The removal of the surface layer of the specimen caused the carbon fibres to expose to wear. Under the contact force, the carbon fibres were partially milled into fine graphite particles. The particles of graphite, which is known as a good solid lubricant, produced the thin films on the wear surfaces of the specimen and its counterface. It is believed that the friction for all the composite specimens is reduced due to the presence of this film, which prevents the metal-to-metal contact between the composite and its counterpart.

The wear losses of the specimens, represented by the volume losses, in the wear tests are illustrated in Fig. 5.9. The corresponding specific wear rates are illustrated in Fig. 5.10. The wear rate of pure Stellite 25 is reduced drastically (up to one order of magnitude) by adding carbon fibres. Specimen $\mathrm{F}$, containing the highest content of 
carbon fibre among the specimens, exhibits the highest wear resistance with a low specific wear rate about $0.86 \times 10^{-5} \mathrm{~mm}^{3} / \mathrm{N} \cdot \mathrm{m}$. The wear resistance of this specimen is improved by about 12 times compared with the pure Stellite 25 specimen. Interestingly, the tribological properties of specimen $F$ are also better than those of Stellite 694 (medium-carbon) and comparable to those of Stellite 712 (high-carbon), which are all fabricated by the sintering/HIPing process and tribologically tested under the same parameter conditions [23].

To further study the tribological behaviour of these new materials, the worn surfaces of the tested specimens were examined using optical and SEM microscopy techniques and the chemical characterization of the worn surfaces were performed by the EDX technique. The images of the wear tracks obtained by optical microscopy are presented in Fig. 5.11. It can be observed that the width of the wear track in the pure Stellite 25 specimen surface is much larger than those of the composite specimens, which is consistent with the high wear rate of pure Stellite 25 . It is also noted that the width of the wear track decreases with the increase of the carbon fibre content in the specimen, which agrees with the wear rate results. In addition, severe wear scars and deep grooves are observed on the surface of this specimen, as shown in the surface image at high magnification (Fig. 5.12). It is also interesting to note that the wear tracks in the composite specimens are intermittent, as shown in Fig. 5.11(b) to Fig. 5.11(f). Compared with the wom surface of pure Stellite 25 specimen in Fig. 5.12, mild and shallow wear scars can be observed in the wear track of the composite specimen at high magnification in Fig. 5.13. The intermittent wear tracks of the composite specimens is definitely 
attributed to the presence of carbon fibres since the friction behavior of Stellite 25 matrix with the counterface differs from that of carbon fibre with the counterface, which results in variation of the friction force along the wear track.

The SEM micrographs and the corresponding EDX spectra of the wear tracks of the pure Stellite 25 , specimen $A$, and the composite specimen $F$ are respectively provided in Fig. 5.14 and Fig. 5.15. While pure Stellite 25 shows severe wear scars on its wear track, as observed in Fig. 5.14(a), the worn surface of specimen F in Fig. 5.15(a) is smooth and the wear track is discontinuous. Also, comparing the EDX spectra in Fig. 5.14(b) and Fig. 5.15(b), in addition to large amounts of $\mathrm{Co}, \mathrm{Cr}$ and $\mathrm{W}$ identified in both surfaces, there is a peak of carbon in the EDX spectrum of specimen F wear track. This verifies that the carbon content on the wear track of specimen $F$ is much higher than that of specimen A. This proves the presence of the carbon-rich film on the wear track of the composite specimens. In addition, the presence of slightly more iron on the wear tracks of the fabricated composites (Fe peak in Fig. 5.15(b)) is due to the transferred debris from the counterpart material (AISI 52100 steel ball). The worn surface of the carbon fibre in the composite specimens was also examined and the corresponding SEM image and EDX spectrum are given in Fig. 5.16. No obvious wear damage other than fibre milling is observed on the carbon fibre surface in Fig. 5.16(a). This very smooth surface further proves the beneficial effect of the carbon fibres on the wear resistance of the reinforced Stellite 25 based composites. The corresponding EDX spectrum in Fig. 5.16(b) indicates a large amount of $\mathrm{C}$ identified in the carbon fibre surface. 


\subsection{Ni-Coated Carbon Fibre Reinforced Composites}

\subsubsection{Microstructure}

The micrographs of the specimens with Ni-coated carbon fibre reinforcement along with the microstructure of the pure Stellite 25 specimen are presented in Fig. 5.17. Similar analyses made on the plain carbon fibre reinforced composites apply to the $\mathrm{Ni}$ coated carbon fibre reinforced composites. Again, it is observed that the pure Stellite 25 specimen and all the fabricated composites exhibit a very similar fine grayish matrix microstructure in the unetched condition. The presence of porosity in the matrices of the fabricated composites is visible, as seen in Fig. 5.18. However, as the amounts of voids are very small, it is reasonable to conclude that the fabrication parameters were selected properly.

With the use of the aforementioned first etching solution, W-rich and Cr-rich carbides can be easily identified in the microstructures due to their blackish colour compared with the matrix colour. In general, there is not much difference in the matrix microstructure of the plain carbon fibre reinforced composites and the Ni-coated carbon fibre reinforced composites. Fig. 5.19 presents some pictures used for carbide fraction analysis of the Ni-coated carbon fibre reinforced composite specimens. The results of carbide volume fraction measurement are shown in Table 5.3. It is shown that the carbide volume fractions in all the specimens are very close. In other words, addition of Nicoated carbon fibres has no significant influence on the carbide volume fraction in the Stellite 25 matrix of the composite specimens. This implies that the nickel coating of the fibres inhibited carbon diffusion at the high temperature during the sintering process. 


\subsubsection{Density}

The specific densities of the Ni-coated carbon fibre reinforced composite specimens are presented in Table 5.4, together with the density value of the pure Stellite 25 specimen for comparison. As expected, the densities of the composites are lower than that of the pure Stellite 25 specimen because the fillers are lighter than the matrix. Also, as previously mentioned, the presence of more voids is inevitable in the composites compared to the pure Stellite 25 specimen due to the incorporation of fibres in the matrix material. Therefore, the density of specimen P containing the highest fibre content is the lowest among the specimens and about $18.5 \%$ less than that of specimen A.

\subsubsection{Corrosion Resistance}

The corrosion rates of the Ni-coated carbon fibre reinforced composite specimens expressed in mils per year are illustrated in Fig. 5.20, together with the corrosion rate value of the pure Stellite 25 specimen for comparison. The results show that the corrosion rate of the pure Stellite 25 specimen is the lowest among the specimens. This behavior is similar to that of the plain carbon fibre reinforced composite specimens. Among the composites, specimen $\mathrm{P}$ that contains the highest carbon fibre content shows the highest corrosion rate with a value of 597 mils/yr. However, compared with the corrosionresistant medium-carbon Stellite alloy, Stellite $6 \mathrm{~B}$, which has a corrosion rate value of 985 mils/yr for the same test condition [76], specimen $\mathrm{P}$ shows better corrosion resistance. Considering the fact that the relationship between the corrosion resistance of Stellite alloys and their carbon content is normally inverse [76], this behavior is more interesting when the carbon contents in Stellite alloys and in the Ni-coated carbon fibre 
reinforced composites are compared. For instance, composite $\mathrm{P}$ contains $10 \mathrm{wt} \%$ carbon fibre (carbon) while Stellite $6 \mathrm{~B}$ contains only $1 \mathrm{wt} \%$ carbon. At $1 \mathrm{wt} \%$ carbon (Stellite 6B), the carbides constitute approximately $13 \mathrm{wt} \%$ of the material, these being predominantly chromium-rich eutectic carbides of the $\mathrm{M}_{7} \mathrm{C}_{3}$ type [79]. Therefore, the carbon in the alloy forms carbides with the alloy elements which deteriorate the corrosion resistance whereas the $\mathrm{Ni}$-coated carbon fibres in the composite do not induce carbides.

As presented in Table 5.3, the Ni-coated carbon fibres induce nearly no carbides in the alloy when they are added into the alloy as the reinforcement even with a high content. Thus, the composites exhibit better corrosion resistance, compared with the Stellite alloys that contain a much lower content of carbon than the content of carbon fibre in the composites, for example, comparing carbon content and corrosion resistance between Stellite 6B and composite specimen P. In addition, the good corrosion resistance of the composites may be attributed to the high corrosion resistance of both Stellite 25 and carbon fibres. It should be again mentioned that the intention for the use of such highly corrosive environment was not to duplicate a practical condition of exposure but to demonstrate the good corrosion resistance of the fabricated composites. This is because corrosion is a complicated process which depends not only on the environment of the exposure but also on the chemical composition of the exposed material.

\subsubsection{Hardness}

The hardness test results of the Ni-coated carbon fibre reinforced composite specimens are illustrated in Fig. 5.21, together with the hardness value of the pure Stellite 
25 specimen for comparison. The hardness of the pure Stellite alloy specimen is enhanced (up to $57 \%$ ) by the addition of Ni-coated carbon fibres. As the fibre content increases the hardness of the composite increases. In particular, composite $\mathrm{P}$, with the largest amount of carbon fibre, exhibits the highest hardness (53.3 HRC). This improvement can be explained by the highest proportion of the fibre which has higher hardness than the matrix material.

\subsubsection{Friction and Wear Resistance}

The variations of friction coefficients with the sliding time for the Ni-coated carbon fibre reinforced composite specimens are presented in Fig. 5.22. The friction coefficient value for the pure Stellite 25 specimen is also presented in the figure for comparison. For each specimen the coefficient of friction increases initially and then decreases until it reaches a nearly constant level, as observed for the plain carbon fibre reinforced composite specimens as well. The constant value of friction coefficient for the pure Stellite specimen is about 0.31 after 55 min sliding, whereas those of the composites vary in a range of $0.14<\mu<0.2$. It is clear that adding carbon fibres in the Stellite alloy greatly reduces the friction. This may be attributed to the formation of a thin solid lubricating film consisting of graphite and metal oxides on the counter surfaces. This behavior is in agreement with the investigations made by other researchers $[42,46,47,48,51]$. Carbon fibre is composed of microcrystals of graphite, which is known as a good solid lubricant. Under the repeating operation of friction force during the sliding process, carbon fibres are milled into fine graphite particles which generate the thin film on the moving surfaces. This film prevents direct metal-to-metal-contact 
between the two counterfaces. The difference in the generation of the thin graphite film between the plain carbon fibre reinforced composites and the $\mathrm{Ni}$-coated reinforced ones is noteworthy. In the latter composites, the graphite particles are exposed and milled only after the $\mathrm{Ni}$-coatings are worn out. Therefore, the graphite films are generated gradually on the moving counterfaces during the wear process.

The wear losses represented by the volume losses of the $\mathrm{Ni}$-coated carbon fibre reinforced composite specimens in the pin-on-disc wear tests are illustrated in Fig. 5.23. The corresponding specific wear rates are reported in Fig. 5.24. The wear values for the pure Stellite 25 specimen are also presented in these figures for comparison. It is shown that addition of $\mathrm{Ni}$-coated carbon fibres can make a remarkable improvement (up to one order of magnitude) in the wear resistance of Stellite 25. Composite $P$ with the highest amount of carbon fibre exhibits the highest wear resistance among all the composites with a specific wear rate of $0.65 \times 10^{-5} \mathrm{~mm}^{3} / \mathrm{N} . \mathrm{m}$. The wear resistance of Stellite 25 is increased by about sixteen times due to addition of Ni-coated carbon fibres up to $10 \mathrm{wt} \%$ in specimen $P$. With the same wear test parameters, the specific wear rate of composite $P$ is less than that of the sintered Stellite 694 (medium-carbon alloy) and comparable with the specific wear rate of the sintered Stellite 712 (high-carbon alloy) [23]. The micrographs of the worn surfaces of the Ni-coated carbon fibre reinforced composite specimens, obtained by optical microscopy, are shown in Fig. 5.25. The micrograph of the wear track of pure Stellite 25 is also presented for comparison. It can be seen that the widths of the wear tracks of the composite specimens are much less than that of the pure Stellite 25 specimen, which correspond to the results of wear loss and specific wear rate. 
It is also observed that the worn surfaces of the composite specimens exhibit obviously less damage than that of the pure Stellite 25 specimen in Fig. 5.25(a). The SEM micrograph and the worn track EDX spectrum of one of the Ni-coated carbon fibre reinforced composite specimens, specimen O, are presented in Fig. 5.26. Compared with the EDX spectrum of specimen A, shown in Fig. 5.14(b), it can be concluded that the concentration of carbon on the wear track of specimen $O$ is much higher than that of specimen A. This would be considered as a proof for the generation of the thin graphite film on the wear track of the composite specimens due to the presence of the nickel coated carbon fibres.

\subsection{Supporting Test Results}

\subsubsection{Microhardness}

In order to further investigate the diffusion of carbon from the carbon fibres into the alloy matrix, microhardness test was conducted on the composite specimens. Specimen $\mathrm{F}$ and specimen $\mathrm{O}$ were selected for these tests since the former contains the highest carbon fibre content among the plain carbon fibre reinforced composite group, while the latter contains the same weight fraction of carbon fibre with specimen $F$ in the $\mathrm{Ni}$-coated carbon fibre reinforced composite group. Four indentations were made at the interface of carbon fibres and the alloy matrix and gradually away to the matrix, as shown in Fig. 5.27. Also, the microhardness of the black carbidic phase in the composite specimens subjected to the standard cobalt-based alloys' etching solution was measured, as shown in Fig. 5.28. The mean microhardness values are presented in Table 5.5. No significant change was observed in the microhardness values obtained at the fibre/matrix 
interface area. Since carbides are much harder than their matrix solid solution, as reported also in literature [1], the test results imply that no major carbide formation occurred in the vicinity of the carbon fibres.

\subsubsection{Thermal Conductivity}

The wear test results demonstrate that carbon fibre has beneficial effects on the tribological properties of the composites. One of its contributions is suggested to be the improved heat transfer, owing to the good thermal conductivity of carbon fibres [57]. To verify this presumption, a comparison study in thermal conductivity on the three specimens, specimen $A$, specimen $F$ and specimen $O$, representing pure Stellite 25,5 wt\% plain carbon fibre reinforced composite and $5 \mathrm{wt} \%$ Ni-coated carbon fibre reinforced composite, respectively, was performed using the pulsed thermography method. Although the actual thermal conductivity values of the specimens cannot be measured with this method, the results can be used as an indication for the heat conduction properties of the specimens. The specimens prepared for this study are shown in Fig. 5.29(a). The PT thermograms of the specimens at $1 \mathrm{~s}$ and $8.15 \mathrm{~s}$ (last frame in the acquisition) are presented in Fig. 5.29(b) and Fig. 5.29(c), respectively. The experimental results are reported in Table 5.6. The $C \times K$ values represent the relative thermal conductivity among the three tested specimens, where the specific heat capacity $(C)$ is assumed to be identical for the three specimens because their base materials are the same.

It is shown that specimen $\mathrm{O}$ has the largest value of $C \times K$ among the three specimens. The values of $C \times K$ are improved $19.3 \%$ and $21.8 \%$ respectively for 
specimens $\mathrm{F}$ and $\mathrm{O}$ compared with that of specimen $\mathrm{A}$. Thus, it can be inferred that adding carbon fibres improves the thermal conductivity of Stellite 25. Comparing composite specimens $\mathrm{F}$ and $\mathrm{O}$, the $C \times K$ value of the latter is $2 \%$ better than the former. Although there are no data about the thermal conductivities of the two different types of reinforcements, this behaviour seems to be reasonable knowing that the thermal conductivity is normally analogous to electrical conductivity $[4,42]$. Thus, the nickel coated carbon fibres which have better electrical conductivity than the plain carbon fibres [60] possess better thermal conductivity as well. The results of the PT test prove the hypothesis that improving the heat dissipation properties of the composite specimens has a direct role in improving their wear resistance. 


\section{Chapter 6: Discussion}

\subsection{Carbide Volume Fraction}

One of the main characteristics of Stellite alloys is their wear resistance. Carbides play the most significant role in the wear resistance of Stellite alloys. However, the formation of unwanted carbidic phases, oxidation of carbides, transformation of carbides, and inhomogeneous distribution of carbidic phases cause many problems in Stellite alloys. Considering these problems associated with the presence of carbon in Stellite alloys, the present research attempted to design and develop new materials, which are Stellite alloy based composites reinforced with carbon fibres. Because of the nature of carbon fibre, the carbon fibre reinforced composites demonstrated their superior tribological properties (lower friction coefficient and higher wear resistance) to Stellite alloys with lower carbon contents but higher carbide volume fractions.

It is worth making the following comparison; for Stellite alloys, at $1 \mathrm{wt} \%$ carbon level (Stellite 6B), the carbides constitute approximately $13 \mathrm{wt} \%$ or $16 \mathrm{vol} \%$ of the material. Also, at a carbon level of $2.4 \mathrm{wt} \%$ (Stellite 3), the carbides constitute about 30 $w t \%$ or 35 vol.\% of the material [1]. However, this research showed that the carbide volume fraction is only $6.4 \%$ for the composite containing $5 \mathrm{wt} \%$ plain carbon fibre plus $0.1 \mathrm{wt} \%$ carbon in its Stellite 25 matrix alloy. In addition, for the composite containing 5 
wt $\% \mathrm{Ni}$-coated carbon fibre plus $0.1 \mathrm{wt} \%$ carbon in the matrix alloy, the carbide volume fraction is even less, only $3.7 \%$. Furthermore, considering composite specimens D and $\mathrm{M}$, which contain $1 \mathrm{wt} \%$ carbon fibre, the carbide volume fractions are $5.2 \%$ and $3.9 \%$ respectively. Therefore, with the same amount of carbon, the carbide volume fraction in the carbon fibre reinforced Stellite alloy composite is much less than that in Stellite alloys. One important feature of the carbon fibre reinforced Stellite alloy composite is that even with much less carbide volume fraction their wear resistance is superior or comparable to commercial medium-carbon Stellite 694 or high-carbon Stellite 712 , respectively [23]. Therefore, in terms of the undesirable effects of carbides, carbon fibre reinforced Stellite alloy composites are much more favorable than the conventional Stellite alloys.

The above comparison reveals that, unlike carbon element, carbon fibre is much more stable at high temperatures in terms of formation of carbides with the alloying elements of Stellite alloys. This is because carbon fibres are chemically inert or unreactive materials [57]. The inertness of carbon fibres makes them stable when they are incorporated with other materials. However, the carbide volume fraction analysis indicated minor increase in carbide volume fraction when plain carbon fibres were added to Stellite 25. This implied that carbon diffusion from the carbon fibres into the Stellite alloy matrix did occur during the $\mathrm{P} / \mathrm{M}$ process. However, the amount of increase in carbide content is trivial compared with that of carbides in medium-carbon and high-carbon Stellite alloys. Nickel coating on the carbon fibres prohibited the carbon 
diffusion even further and made the use of carbon fibres more attractive in consideration of carbide formation.

\subsection{Beneficial Effects of Carbon Fibres}

The results of the density test showed that the densities of the composites are lower than that of the pure Stellite 25 specimen since carbon fibres are much lighter than the Stellite 25 alloy matrix. In particular, specimens $F$ and $P$ are respectively $14 \%$ and $18.5 \%$ lighter than specimen A that might be interpreted as higher structural efficiency for the composites compared with the pure Stellite 25 alloy.

Carbon fibre is known as a reinforcing material that can induce good mechanical properties and wear resistance in composites so that it is expected to be comparable to the carbides in Stellite alloys in terms of these properties while the drawbacks of the carbides, as mentioned earlier, could be eliminated. The corrosion and wear tests in this research demonstrated that the carbon fibre reinforced Stellite alloy composites possess superior properties to conventional Stellite alloys.

The corrosion test showed that both the plain carbon fibre reinforced and the $\mathrm{Ni}$ coated carbon fibre reinforced composites had better corrosion resistance than mediumcarbon and high-carbon Stellite alloys. The reason lies in the fact that the key factor affecting the corrosion behaviour of Stellite alloys is the carbides precipitated in the alloy matrices due to the presence of carbon. This is because of chemical micro-segregation and also shortage of vital corrosion resistant alloying elements such as chromium in the 
matrix of Stellite alloys after formation of carbides. Therefore, the amount of carbides present in the alloys controls their corrosion performance. Owing to the homogeneous microstructure of their composite matrices with minor carbides and more chromium in the solid solution, the fabricated carbon fibre reinforced materials exhibited better corrosion resistance compared with medium- and high-carbon Stellite alloys.

The corrosion mechanisms of the fabricated composites include galvanic corrosion and aqueous corrosion (surface oxidation). Galvanic corrosion happens because dissimilar materials possessing different surface potential are electrically coupled in the conductive corrosive media [46]. While the conductive corrosive media is the dilute boiling nitric acid, the dissimilar materials include the matrix alloy, conducting non-metal of carbon fibres and the nickel coating on the carbon fibres. Since graphite (carbon fibre) is placed almost at the top of the galvanic series as one of the noblest materials, the matrix material becomes anodic to the fibres.

Another involved corrosion mechanism in these composites is aqueous corrosion that is caused by electrochemical reactions between the corrosive media of dilute boiling nitric acid, the matrix material and the fibres resulting in a form of degradation in the composites. It can be observed in Fig. 5.6 and Fig. 5.20 that the corrosion rate increases with increasing the fibre content. This can be explained by the increased contact area between the corrosive media and the composite materials due to the higher void content in the composites. Also, raising the carbon fibre content increases the interface area between the alloy matrix and the fibre. 
Hardness is one of the important factors in the characterization of a material. In particular, hardness is used quite often in the field of wear resistance as a qualitative indicator for judging alloys, coatings, hardfacings and overlays. It was shown in this research that adding carbon fibres enhanced the hardness of Stellite 25 and the hardness increased with the increase of carbon fibre content in the composite. This is understandable because the hardness of the reinforcing elements is a leading factor that affects the hardness of the composites. It is interesting to compare hardness between the carbon fibre reinforced Stellite alloy composites and conventional medium-carbon Stellite alloys or high-carbon Stellite alloys. For instance, high-carbon Stellite alloys such as Stellite 1, Stellite 3, Stellite 20 and Stellite 720 that contain a similar amount of carbon (about $2.5 \mathrm{wt} \%$ ) have a hardness value around $\mathrm{HRC} 60$, and medium-carbon Stellite alloys such as Stellite 6, Stellite 694 and Stellite 706 with 1 wt $\% \sim 1.2$ wt $\%$ carbon have a hardness value between HRC 40 and HRC 50 [1]. However, from the hardness test results in Fig. 5.7, specimen F containing $5 \mathrm{wt} \%$ carbon fibre has a hardness value of HRC 50.5, and specimen D and specimen E containing $1 \mathrm{wt} \%$ and $2 \mathrm{wt} \%$ carbon fibre, respectively, have the corresponding hardness values of HRC 44.6 and HRC 49.4, accordingly.

This comparison shows that the influence of carbon fibre content on the hardness for the composites is not as significant as carbon content on the hardness for Stellite alloys. This is not surprising if the carbide amount in Stellite alloys is considered. As was well known, the hardness of Stellite alloys is predominated by the amount of carbides precipitated in the solid solution matrix, except for low-carbon Stellite alloys [1]. For the 
Stellite alloys containing about $2.5 \mathrm{wt} \%$ carbon, the carbides constitute about $30 \mathrm{wt} \%$ of the materials, corresponding to the volume fraction between $30 \% \sim 40 \%[1]$, which is much larger than the volume fraction of the carbon fibres in specimen F (19.6\% shown in Table 3.8). However, from the above comparison, it is worth noting that the composite containing the same amount of carbon fibre as the amount of carbon in Stellite alloys has comparable hardness to the Stellite alloys, while the carbide volume fraction in the composite is much less than that in the Stellite alloys.

The repair and replacement of damaged parts caused by the dimensional size loss because of wear are very costly in industry. The tribological tests demonstrated that adding carbon fibres reduced the friction coefficient and significantly increased the wear resistance of Stellite 25. Also, compared with conventional Stellite alloys, the carbon fibre reinforced Stellite alloy composites containing $5 \mathrm{wt} \%$ plain carbon fibre and 10 $w t \% \mathrm{Ni}$-coated carbon fibre possess better wear resistance than those of medium-carbon Stellite alloys and comparable to those of high carbon Stellite alloys. The factors contributing to these improved features can be summarized as follows:

\section{(1) Graphite film formation:}

Friction is known as the force resisting the sliding of one solid body over or along another body. The resistant force tends to oppose the relative motion of the two moving surfaces in mechanical contact $[26,28,74]$. The causes for the formation of this resistant force, which commonly occurs in machinery, include a set of microscopic interactions between the moving surfaces. These interactions are 
influenced by physical, geometrical, mechanical and chemical characteristics of the sliding surfaces, their surrounding atmosphere, and overall functional sliding conditions. When two surfaces are in contact with each other, the actual mechanical contact area comprises a number of small local asperities. Thus, the actual contact area is much smaller than the nominal contact area that leads to an undesirable wear and surface damage caused by the friction force $[26,28,74]$. Therefore, various liquid lubricants including water, oil, and grease are used to reduce the friction and consequently wear by providing easier sliding.

Although they could efficiently control the system friction, liquid lubricants have usage constraints for many reasons including complicated housing design problems for oilways, accelerated corrosion damage in hostile environments, environmental considerations, and operational problems [26]. To this end, there is a strong desire among industrial designers to produce self-lubricating materials that effectively lessen friction without employing any external liquid lubricants. Self-lubricants are any solid materials that show low friction in motion without application of liquid lubricants. Graphite, molybdenum disulfide $\left(\mathrm{MoS}_{2}\right)$, boron nitride $(\mathrm{BN})$, and polytetrafluoroethylene (PTFE) are the predominant materials used as solid lubricants. These materials are introduced between two rubbing surfaces for the purpose of reducing friction and providing protection from damages that happen during relative movement $[26,28,74]$. 
Friction is also known as a course of energy transformation. According to the law of conservation of energy, in the rubbing process of the sliding surfaces some of the energy is transformed into heat, which can deteriorate the strength of the surface materials, thus the wear resistance of the surfaces. The formation of the graphite film on the carbon fibre reinforced Stellite alloy composites and their mating surfaces, as proved by the worn surface analysis, not only reduces the friction by increasing the real contact moving area and providing solid lubrication but also lowers the friction heat generated in the rubbing process by providing easier sliding where metal-tometal contact is avoided. Thus, adhesive wear due to the localized bonding between the contacting asperities is mitigated in the composites. In addition, the graphite film on the composite surfaces also inhibits abrasive wear since the friction force is not large enough to pull out the fibres from the Stellite alloy matrix. Therefore, compared with pure Stellite 25 alloy, it can be concluded that due to the presence of the carbon fibres the wear resistance of the fabricated composites is enhanced while their friction coefficient is reduced.

\section{(2) Abrasion resistance improvement:}

The increase in wear resistance of the composites can be also attributed to the abrasion reducing role of carbon fibres. Since the dominant wear mechanism of sliding wear is abrasion, the contact surfaces are normally damaged by groove ploughing, as shown in Fig. 5.11(a) and Fig. 5.25(a). Without carbon fibre reinforcement, specimen A surface suffered severe abrasive wear, characterized by numerous long and deep grooves which corresponded to a large volume of material 
being scratched out from the surface, as shown in Fig. 5.12. This type of abrasion is promoted in Stellite alloys due to the entrapment of carbide particles between contact surfaces in relative motion. When removed by micro cutting from the matrix of Stellite alloys, carbides, which are super hard particles, can plough both the matrix and their opposing moving surfaces. The produced debris can even further aggravate the abrasive wear between the two metallic surfaces as a third body.

On the contrary, the detached small particles of carbon fibres are milled into soft graphite powder during the sliding process. In this case, the new reinforcing elements (carbon fibres) not only improve surface abrasion resistance by eliminating carbides, but also provide surface lubrication. Both result in less groove ploughing, less material defragmentation, and much narrower wear tracks in composites compared with pure Stellite 25 alloy, which can be clearly observed in Fig. 5.11(b) to Fig. 5.11(f) and Fig. 5.25(b) to 5.25(g). Therefore, in light of the much lower wear rates offered by the fibres, it can be concluded that the fibres might have a wearpreventive role by acting as hard barriers to control the size and the shape of the wear fragments in the composites. In addition, no abrasive wear due to the pulled out fibre from the matrix, caused by the mechanical attack during the sliding process, was observed in the wear test. This implies that the interface bonding between the matrix and the fibres in the composites is good. 


\section{(3) Fibre/matrix interface compatibility:}

The interface bonding between the reinforcement and the matrix is always a major consideration for composite materials, because the failure of the interface results in spallation of the reinforcement from the matrix under mechanical attack. For wear resistance applications, the spallation of the reinforcement from the matrix under the wear load greatly increases the wear loss of the material. For the composites fabricated in this research, no carbon fibre spallation is observed from the worn surface analysis. Further investigation of the interface bonding behaviour of the composite specimens was performed utilizing a Vickers hardness tester. The indentations were made with the sharp corner diamond pyramid indenter of the tester in the locations close to and at the fibre-matrix interface. If the interface is weak, under the indentation load the sharp indent would initiate cracks at the interface or in the matrix. A load level of $60 \mathrm{~kg}$ was used for this purpose; however, no cracking was observed on any of the composite specimens. The samples of the indentation images are presented in Fig. 6.1 and Fig. 6.2. Although this test did not provide a quantitative estimation of the interface bonding strength of the composite specimens, it indicated indirectly that the interface bonding of the composites is strong.

The HIP/sintering process employed for fabricating the specimens and the compatibility of the matrix material with the reinforcing elements may be the main contributing factor to the strong interface bonding of the composites. The term "compatibility" in metal matrix composites means that no undesirable chemical reaction is experienced at the matrix/reinforcement interface [31]. These reactions 
may have deleterious effects on mechanical and tribological properties of MMCs by forming detrimental products such as intermetallic compounds and carbidic phases at the matrix/reinforcement interface. The unwanted intermetallic and carbidic products not only reduce the matrix to reinforcement load transferring capability of MMCs but also act as crack nucleation sites [42]. The microhardness test results on the composite specimens in Table 5.5 show that hardness is fairly constant around the interface and identical to the overall matrix hardness. This also indicates indirectly that no detrimental products were formed at the fibre-matrix interface of the composites in the fabrication process. The two experiments mentioned in this section support the wear test observation that the interface of the composites is strong enough to avoid the carbon fibre pull-out from the matrix under the wear load.

\section{(4) Hardness augmentation:}

Although no single definable material property can explain wear behaviour, it is believed that the wear resistance of a material is affected by its hardness. In general, increasing the hardness of a material enhances its wear resistance since with the higher hardness the material is the less prone to deform plastically $[1,3,9,28,48]$. The experimental results show that the hardness of Stellite 25 is enhanced by the addition of carbon fibres. It is evident that the hardness induced by carbon fibres contributes to the high wear resistance of the carbon fibre reinforced Stellite alloy composites. 


\section{(5) Heat dissipation improvement:}

If the heat generated due to friction during the sliding process is not properly dissipated, the temperature rise can change the surface property of the rubbing counterfaces that results in more adhesive wear $[28,42,80]$. Another noticeable reason that the carbon fibre reinforced composites exhibited better tribological properties than their matrix materials might lie in the improved thermal conductivity of the composites. Due to their excellent thermal conductivity, carbon fibres can serve not only as reinforcement, but also as an additive to improve the thermal conductivity of the composites [57]. Since these fibres have higher thermal conductivity than the alloy matrix $[22,42,46,57,58,60]$, the presence of the fibres can reduce the surface temperature during wear and thus enhance the resistance to wear. This is more evident knowing that increasing the content level of carbon fibres results in escalating their wear resistance. Such hypothesis was also discussed by other researchers for carbon fibre reinforced composites including discontinuous fibre reinforced aluminum alloy composites (DFRACs) $[28,42,46,51,80]$.

The comparison study of thermal conductivity of pure Stellite 25 , plain carbon fibre reinforced composite and $\mathrm{Ni}$-coated carbon fibre reinforced composite was performed in this research. The results, presented in Table 5.6, showed that the relative thermal conductivity of pure Stellite 25 was improved by $19.3 \%$ and $21.8 \%$ with addition of plain carbon fibre and $\mathrm{Ni}$-coated carbon fibre, respectively. Therefore, it can be concluded that carbon fibres also improve the thermal 
conductivity of the composites, thus reducing the friction heat in the wear process and resultant wear damage.

\subsection{Effects of Nickel Coating}

Two types of carbon fibre were selected to reinforce the composites fabricated in this research: plain carbon fibre and $\mathrm{Ni}$-coated carbon fibre. The purpose of using these two different carbon fibres was to investigate if nickel coating would benefit the performance of the composites.

\section{(1) Carbide volume fraction:}

The carbide volume fraction analysis indicated that $\mathrm{Ni}$ coating on the carbon fibres, as an isolator between the carbon fibre and the alloy matrix, did prohibit carbon diffusion, which avoided formation of carbides. In other words, the Ni-coating on carbon fibres can lead to elimination of the detrimental effects of carbides in Stellite alloys, when carbon fibres are incorporated.

\section{(2) Density:}

As shown in Fig. 6.3, with the same weight content of carbon fibre, the composite reinforced with $\mathrm{Ni}$-coated carbon fibres has higher specific density than that reinforced with plain carbon fibres. This difference may exist because of two reasons: firstly, the density of Ni-coated carbon fibre is much higher than that of plain carbon fibre (about $50 \%$ ), as presented in Table 3.4 and Table 3.6. Secondly, the Ni-coating on carbon fibre may have reduced porosity of the composites which usually develops during the sintering 
process. The less porous structure of the Ni-coated fibre reinforced composites can be explained by the presence of the Ni-coating which acts as the interface between the alloy matrix and the carbon fibre. Since nickel is more cohesive to Stellite 25 than carbon fibre, the cohesion of the two dissimilar constituent materials (carbon fibre and Stellite 25) in Ni-coated carbon fibre reinforced composites might be enhanced.

\section{(3) Corrosion resistance:}

The comparison of corrosion resistance between the plain carbon fibre reinforced composites and the $\mathrm{Ni}$-coated carbon fibre reinforced composites is illustrated in Fig. 6.4. The results show that the plain carbon fibre reinforced composites possess better corrosion resistance than the Ni-coated reinforced composites. The rate of galvanic corrosion is usually determined by the difference in nobility of materials [71]. In the galvanic series, nickel is placed lower than graphite (carbon fibre). Therefore, the nickel coating becomes anodic to the fibres. Also, between the nickel coating and Stellite 25 alloy, the matrix material becomes cathodic. Thus, nickel coating takes the form of galvanic anode to control the corrosion of the matrix by means of cathodic protection. Therefore, the galvanic corrosion of Stellite 25 only happens after the nickel coating decays. The high corrosion rates in the $\mathrm{Ni}$-coated composites compared with the plain carbon fibre composites can be explained by the virtue of the more galvanic corrosion of nickel in the former composites. 


\section{(4) Hardness:}

The hardness values of the two groups of composites are compared in Fig. 6.5. With the same content of carbon fibre, the hardness values of the composites reinforced with plain carbon fibres are higher than those of the composites reinforced with $\mathrm{Ni}$ coated carbon fibres. The most pronounced reason for such difference is that the $\mathrm{Ni}$ coating prohibited carbon diffusion from the carbon fibres into the Stellite alloy matrix during the specimen fabrication process so that the formation of new carbides was suppressed. Therefore, the overall volume fraction of hard carbidic compounds in the plain carbon fibre reinforced composites is relatively higher than that in the Ni-coated carbon fibre reinforced composites which results in higher hardness values in these composites.

\section{(5) Friction and wear resistance:}

The comparisons of friction and wear resistance between the two groups of composites are illustrated in Fig. 6.6 and Fig. 6.7, respectively. It is shown that the plain carbon fibre reinforced composites possess better tribological properties. As observed, the friction coefficient of the plain carbon fibre reinforced composites varies in a range of $0.13<\mu<0.16$, while that of the Ni-coated carbon fibre reinforced composites varies in a range of $0.14<\mu<0.2$. The higher friction force in the latter composites is due to the fact that the lubricating graphite film is generated gradually on their moving counterfaces during the wear process only after the Ni-coating is worn out and the graphite particles are milled between the asperities. 
Considering the aforementioned factors influencing the tribological properties of the fabricated composites, it can be noticed that even though the Ni-coated reinforced composites possess relatively better thermal conductivity, they have some downsides including higher friction coefficient values due to the delay in formation of graphite film layer on the specimen surface, lower overall hardness, and higher abrasive wear rate because of the presence of the milled nickel coating particles. The reduction in wear resistance of the composites reinforced with $\mathrm{Ni}$-coated carbon fibres compared with those reinforced with plain carbon fibres may have two other reasons. Firstly, the carbide volume fraction in the former is less than that in the latter when their carbon fibre contents are the same. The less carbide in the Ni-coated carbon fibre reinforced composites results in the reduction in wear resistance of the matrix that eventually leads to the increased wear rate of the composites. Secondly, the higher friction coefficient of the Ni-coated carbon fibre reinforced composites is another reason for their higher wear rate.

\section{(6) Thermal conductivity:}

The thermal conductivity test showed that the $C \times K$ product for composite specimen $\mathrm{O}$ (with Ni-coated carbon fibre) is $2 \%$ higher than that for the composite specimen F (with plain carbon fibre). Therefore, it can be inferred that the relative thermal conductivity of the Ni-coated carbon fibre reinforced composites is better than that of the plain carbon fibre reinforced composites. Considering that the volume fraction of carbon fibres in composite $O(13.9 \mathrm{vol} \%)$ is lower than that in composite $F(19.6$ vol.\%), it can be inferred that the nickel coating increases the thermal conductivity of 
carbon fibres. This statement is in agreement with the general behavior of metallic materials for which the best electrical conductors are the best thermal conductors as well $[4,42]$.

Although the present research is not aimed at recommending the fabricated composites for any specific application, it is worth comparing the properties of these composites with self-lubricating slider bearing materials. Under the same conditions of exposure, the fabricated composites show better tribological properties and corrosion resistances than PTFE based sliding bearing materials [28]. As presented in Table 6.1, while maintaining the super low coefficient of friction of PTFE because of the lubricating effect of the thin transfer film of graphite, the fabricated composites possess superior corrosion and wear resistance than those of PTFE based composites. Therefore, one main application for the fabricated composites can be in the bearing industry. The formation of graphite film on the composites and their sliding counterfaces can ensure the workability of the potential bearings in applications where the thin-film lubrication or boundary lubrication regimes are predominant. 


\section{Chapter 7: Conclusions and Future Work}

\subsection{Conclusions}

Two groups of carbon fibre reinforced Stellite alloy composites have been designed and developed in this research. Conventional Stellite 25 was selected as the matrix of the fabricated composites because it contains very low carbon content. The reinforcing elements included plain carbon fibres and nickel-coated carbon fibres. The specimens were produced successfully by the sintering/hot isostatic pressing routine. Based on the experimental results and the discussion, the following conclusions can be drawn from this research:

1. The pure Stellite 25 specimen and all the composite specimens have a very similar matrix microstructure comprising of three different phases. These phases include the cobalt solid solution, the tungsten-rich carbide and the chromium-rich carbide.

2. The presence of carbon fibres increases slightly the carbide volume fraction of the plain carbon fibre reinforced composites compared with that of pure Stellite 25 alloy. This implies that carbon fibres are stable at high temperatures and differ from carbon which forms carbides with metallic 
elements. This feature of carbon fibres avoids the undesirable effects of carbides in Stellite alloys.

3. The carbide volume fractions in all the Ni-coated carbon fibre reinforced group specimens are nearly equal, which indicates that the carbon did not diffuse from the fibres into the alloy matrix to induce carbides at the high temperature during the sintering process. The diffusion deterrence may be attributed to both the high-temperature stability of carbon fibres and the nickel coating.

4. The sintering/hot isostatic pressing process employed in this research produced the specimens with low porosity, thus achieving fairly high density. However, the composite specimens have lower density than that of the pure Stellite 25 specimen, owing to the light weight nature of carbon fibres.

5. Addition of carbon fibre into the low-carbon Stellite alloy, Stellite 25, enhances its hardness and improves its tribological properties significantly. Both the hardness and wear resistance of the fabricated composites increase with the content of the carbon fibre added.

6. The superior tribological properties of carbon fibre reinforced Stellite alloy composites to those of Stellite alloys are ascribed to three beneficial effects of carbon fibre on these alloys: (1) increased hardness, (2) reduced friction due 
to the formation of graphite film on the composite surface during wear, and (3) improved thermal conductivity and hence reduced friction heat.

\subsection{Contributions}

The novel manufactured composites solve the main problems caused by the carbides in Stellite alloys and exhibit superior tribocorrossion properties to these alloys. The main contributions of this research can be outlined as follows:

1. The fabricated composites, even the specimens with the highest content of carbon fibre, exhibit better corrosion resistance than medium-carbon Stellite alloys, for example, Stellite 6B. This is attributed to the beneficial feature of the carbon fibre reinforcement that induces either no or little amount of carbides in the alloy matrix.

2. The tribological properties of the composite with $5 \mathrm{wt} \%$ plain carbon fibre and the composite with $10 \mathrm{wt} \% \mathrm{Ni-coated}$ carbon fibre are better than those of medium-carbon Stellite alloys and comparable to those of high-carbon Stellite alloys.

3. The newly fabricated carbon fibre reinforced composite materials avoid the detrimental effects of carbides in Stellite alloys while improving/maintaining their good properties. Therefore, these composites can become a new generation of self-lubricating wear/corrosion resistant materials for various 
industrial applications. The new composites can find applications in gas turbine, chemical and petrochemical industries for instance where components such as pumps and impellers must withstand the simultaneous wear and corrosion.

\subsection{Future Work}

Following the studies described in this thesis, a number of issues should be explored in the future work including:

1. The newly fabricated materials should also be fabricated with casting techniques. For this fabrication method, optimum process parameters should be identified.

2. The cast specimens should be characterized in terms of the microstructure, hardness, corrosion and wear resistance. It is also important to compare these properties with those of the $\mathrm{P} / \mathrm{M}$ specimens characterized in this research.

3. The effects of the shape, size and aspect ratio of the filler (carbon fibre) on the performance of the composites should be investigated by using non-bundle carbon fibres.

4. Further investigation of the performance of carbon fibre in the composites could be accomplished by selecting a carbon-free Stellite alloy instead of the lowcarbon Stellite 25 alloy as the matrix in order to eliminate the influences of the 
carbides in Stellite alloys on the final properties of the composites or to mitigate the problems caused by carbides in the final composites.

5. Material characterization tests on these new composites can be conducted at elevated temperatures to study the functionality of these materials in more severe environments. 


\section{References}

[1] ASM Handbook Committee, "Nickel, Cobalt, and Their Alloys", ASM International, Cleveland, USA, 2000.

[2] W. Betteridge, "Cobalt and its Alloys", Ellis Horwood Ltd., Cambridge, England, 1982.

[3] R. Liu, M.X. Yao, and X. Wu, "Influence of Carbon Content in Cobalt-Based Superalloys on Mechanical and Wear Properties", Journal of Engineering Materials and Technology, Vol. 126 (2), 2004, pp. 204-12.

[4] J.R. Davis, "Metals Handbook Desk Edition", $2^{\text {nd }}$ Edition, ASM International, Cleveland, USA, 1998.

[5] M.J. Donachie and S.J. Donachie, "Superalloys, A Technical Guide", Second Edition, ASM International, Cleaveland, USA, 2002.

[6] J. Shin, J. Doh, J. Yoon, D. Lee, and J. Kim, "Effect of Molybdenum on the Microstructure and Wear Resistance of Cobalt-Base Stellite Hardfacing Alloys", Surface and Coatings Technology, Vol. 166, 2003, pp. 117-26. 
[7] P. Waldner, E. Knigsberger, and H. Gamsjfiger, "Computer-Assisted Optimization of Cobalt-Base Alloy Compositions", Journal of Alloys and Compounds, Vol. 220, 1995, pp. 148-51.

[8] D. Lou, J. Hellman, J. Limatainen, and V.K. Lindroos, "Interactions between Tungsten Carbide (WC) Particulates and Metal Matrix in WC-Reinforced Composites", Materials Science and Engineering A, Vol. 340, 2003, pp. 155-62.

[9] W.H. Jiang, X.D. Yao, H.R. Guan, and Z: Q. Hu, "Carbide Behavior during High Temperature Low Cycle Fatigue in a Cobalt-Base Superalloy", Journal of Materials Science, Vol. 34, 1999, pp. 2859-64.

[10] W. Jiang, $\mathrm{X}$. Yao, and Z. Hu, "Secondary $\mathrm{M}_{6} \mathrm{C}$ Precipitation in a Cobalt-Base Superalloy”, Journal of Materials Science Letters, Vol. 18, 1999, pp. 303-5.

[11] ASM Handbook Committee, "Powder Metal Technologies and Applications", Vol. 7, ASM International, Cleveland, USA, 1998.

[12] ASTM Standard Committee, "Standard Test Method for Density of Powder Metallurgy (PM) Materials Containing Less Than Two Percent Porosity", (B 311-08), ASTM International, USA, 2009. 
[13] ASTM Standard Committee, "Standard Practice for Preparing, Cleaning, and Evaluating Corrosion Test Specimens", (G1-03), ASTM International, USA, 2007.

[14] ASTM Standard Committee, "Standard Practice for Laboratory Immersion Corrosion Testing of Metals", (G 31-72), ASTM International, USA, 2004.

[15] ASTM Standard Committee, "Standard Test Methods for Rockwell Hardness of Metallic Materials", (E 18-08b), ASTM International, USA, 2009.

[16] ASTM Standard Committee, "Standard Test Method for Wear Testing with a Pin-on-Disk Apparatus", (G 99-05), ASTM International, USA, 2005.

[17] F.C. Campbell, "Manufacturing Technology for Aerospace Structural Materials", Elsevier Science and Technology Books, St. Louis, USA, 2006.

[18] R.C. Reed, "The Superalloys Fundamentals and Applications", $1^{\text {st }}$ Edition, Cambridge University Press, Cambridge, UK, 2006.

[19] A. Beltran, E. Brown, W. Chambers, and D. Chang, "Superalloys II", Wiley Inc., New York, USA, 1987. 
[20] A. Raman, "Materials Selection and Applications in Mechanical Engineering", Industrial Press, New York, USA, 2007.

[21] R.S. Young, "Cobalt, Its Chemistry, Metallurgy, and Uses", Reinhold Publishing Corporation, New York, USA, 1960.

[22] ASM Handbook Committee, "Properties and Selection: Nonferrous Alloys and Special-Purpose Materials", Vol. 2, 10 $0^{\text {th }}$ Edition, ASM International, Cleveland, USA, 1990.

[23] C. Opris, R. Liu, X. Yao, and X. Wu, "Development of Stellite Alloy Composites with Sintering/HIPing Technique for Wear-Resistant Applications", Materials and Design, Vol. 28, 2007, pp. 581-91.

[24] Y.Q. Wang and B.L. Zhou, "Behavior of Coating on Reinforcements in Some Metal Matrix Composites”, Composites Part A, 27A, 1996, pp. 1139-45.

[25] D. Raghu and J. B. Wu, "Recent Development in Wear and CorrosionResistant Alloys", Materials Performance, Vol. 36, 1997, pp. 27-36.

[26] A. Khoddamzadeh, "Development of Lead-Free PTFE Based Sliding Bearing Materials", M.A.Sc Materials, Department of Mechanical and Aerospace Engineering of Carleton University, Ottawa, Canada, 2005. 
[27] W. Xu, "The Influence of Chemical Composition and Heat Treatment on Microstructure and Mechanical/Tribological Properties of Cobalt-Based Tribaloy Alloys", Department of Mechanical and Aerospace Engineering of Carleton University, Ottawa, Canada, 2005.

[28] A. Khoddamzadeh, R. Liu, and X. Wu, "Novel Polytetrafluoroethylene (PTFE) Composites with Newly Developed Tribaloy Alloy Additive for Sliding Bearings", Wear Journal, Vol. 266, 2009, pp. 646-657.

[29] M.X. Yao, J. Wu, and Y. Xie, "Wear, Corrosion and Cracking Resistance of Some W- or Mo-Containing Stellite Hardfacing Alloys", Materials Science and Engineering A, Vol. 407, 2005, pp. 234-44.

[30] C. Sims, N. Stoloff, and W. Hagel, "Superalloys II: High Temperature Materials for Aerospace and Industrial Power", John Wiley \& Sons, Toronto, Canada, 1987.

[31] A. Frenk and J. D. Wagnière, "Laser cladding with cobalt-based hardfacing", Journal de Physique IV, 1991, pp. 65-8.

[32] Z. Oksiuta, J.R. Dabrowski, and A. Olszyna, "Co-Cr-Mo-Based Composite Reinforced with Bioactive Glass", Journal of Materials Processing Technology, Vol. 209, 2009, pp. 978-85. 
[33] S. C. Lim and M. F. Ashby, "Wear-Mechanism Maps", Acta Metallurgica, Vol. 35,1987, pp. 1-24.

[34] A. Frenk and W. Kurz, "Microstructural Effects on the Sliding Wear Resistance of a Cobalt Based Alloy", Wear Journal, Vol. 174, 1994, pp. 81-91.

[35] C. J. Heathcock, A. Ball, and B. E. Protheroe, "Cavitation Erosion of CobaltBased Stellite Alloys, Cemented Carbides and Surface-Treated Low Alloy Steels", Wear Journal, Vol. 74, 1981, pp. 11-26.

[36] A. Davin and D. Coutsouradis, "Development of Abrasion- and CorrosionResistant Alloys for Use in Aqueous Media", Cobalt Journal, Vol. 52, 1971, pp. $160-1$.

[37] D. L. Klarstrom, "Wrought Cobalt-Base Superalloys", Journal of Material Engineering Performance, Vol. 2, 1993, pp. 523-30.

[38] L. C. Wang and D. Y. Li, "Effects of Yttrium on Microstructure, Mechanical Properties, and High Temperature Wear Behaviour of Cast Stellite 6 Alloy", Wear Journal, Vol. 255, 2003, pp. 535-44.

[39] I. Radu, D. Y. Li and R. Llewellyn, "Tribological Behaviour of Stellite 21 Modified with Yttrium", Wear Journal, Vol. 257, 2004, pp. 1154-66. 
[40] G. Y. Lai, J. J. Barnes, and J. E. Barnes, "A Burner Rig Investigation of the Hot Corrosion Behaviour of Several Wrought Superalloys and Intermetallics", ASME, New York, USA, 1991.

[41] F.R. Morral, "Cobalt-Base Superalloys Produced from Powder", Planseeberichte fr Pulvermetallurgie, Vol. 20, 1972, pp. 3-19.

[42] ASM Handbook Committee, "Composites", Vol. 21, ASM International, Cleveland, USA, 2001.

[43] N. Chawla and K. Chawla, "Metal Matrix Composites", Springer, New York, USA, 2006.

[44] B. Ralph, H.C. Yuen, and W. B. Lee, "The Processing of Metal Matrix Composites-An Overview", Journal of Materials Processing Technology, Vol. 63, 1997, pp. 339-53.

[45] Y. Q. Wang, B.L. Zhou, "Behavior of Coating on Reinforcements in Some Metal Matrix Composites", Composites Part A, 27A, 1996,pp. 1139-45.

[46] L. Liu, T. Ping, and S. Bin, "Friction and Wear Properties of Short Carbon Fibre Reinforced Aluminum Matrix Composites", Wear, Vol. 266, 2009, pp. 733-8. 
[47] J. Song and K. Had, "Effect of Volume Fraction of Carbon Fibres on Wear Behavior of $\mathrm{Al} / \mathrm{Al}_{2} \mathrm{O}_{3} / \mathrm{C}$ Hybrid Metal Matrix Composites", Composite Structures, Vol. 39, 1997, pp. 309-318.

[48] D. Jun, L. Yaohui, Y. Sirong, and D. Handa, "Effect of Fibre-orientation on Friction and Wear Properties of $\mathrm{Al}_{2} \mathrm{O}_{3}$ and Carbon Short Fibres Reinforced AlSi $_{12}$ CuMgNi Hybrid Composites", Wear Journal, Vol. 254, 2003, pp. 164 72.

[49] S. Li and C. Chao, "Effects of Carbon Fibre/Al Interface on Mechanical Properties of Carbon-Fibre-Reinforced Aluminum-Matrix Composites", Metallurgical and Materials Transactions A, Vol. 35, 2004, pp. 2153-60.

[50] A. Diwanji and I. Hall, "Fibre and Fibre-Surface Treatment Effects in Carbon/Aluminium Metal Matrix Composites", Journal of Materials Science, Vol. 27, 1992, pp. 2093-100.

[51] A. Daoud, "Wear Performance of 2014 Al Alloy Reinforced with Continuous Carbon Fibres Manufactured by Gas Pressure Infiltration", Materials Letters, Vol. 58, 2004, pp. 3206-13.

[52] L. Kong, Y. Lu, and B. Yang, "Investigation of Friction and Wear Behaviour of Carbon Fibre Felt Reinforced Aluminium-Matrix Composites", Tribology, Vol. 
21,2001, pp. $448-451$.

[53] C.S. Lee, Y.H. Kim, and K.S. Han, "Wear Behaviour of Aluminium Matrix Composite Materials”, Journal of Materials Science, Vol. 27, 1992, pp. 793-800.

[54] J. Xu, H. Yu, L. Xia, and H. Yang, "Effects of Some Factors on the Tribological Properties of the Short Carbon Fibre-Reinforced Copper Composite", Materials \& Design, Vol. 25, 2004, pp. 489-93.

[55] L. Xia, B. Jia, J. Zeng, and J. Xu, "Wear and Mechanical Properties of Carbon Fibre Reinforced Copper Alloy Composites", Materials Characterization, Vol. 60, 2009 , pp. 363-369.

[56] Z. Jun, X. Jincheng, H. Wei, and L. Lei, "Wear Performance of the Lead Free Tin Bronze Matrix Composite Reinforced by Short Carbon Fibres", Applied Surface Science, Vol. 255, 2009, pp. 6647-51.

[57] Zoltek Committee, “User's Guide for Short Carbon Fibre Composites", Zoltek Companies Inc., St. Louis, USA, 2000.

[58] Deloro Stellite Committee, "Stellite 25 Technical Data", Deloro Stellite Company, Goshen, USA, 2009. 
[59] J. Davies, "Cobalt Base Alloys MSDS", Deloro Stellite Company, Goshen, USA, 2007.

[60] Toho Tenax Committee, "Tenax Chopped Products Technical Data", Toho Tenax America, Inc., Rockwood, USA, 2006.

[61] G. Hohne and W. Hemminger, "Differential Scanning Calorimetry", $2^{\text {nd }}$ Edition, Springer, Berlin, Germany, 1996.

[62] ASTM Standard Committee, "Standard Terminology Relating to Thermal Analysis and Rheology", (E 473 - 08), ASTM International, USA, 2008.

[63] J. Overton, "Thermophysical Property and Phase Transformation Determination of TiAl Intermetallics", M.A.Sc, Department of Mechanical and Aerospace Engineering of Carleton University, Ottawa, Canada, 2006.

[64] ASTM Standard Committee, "Standard Test Method for Enthalpies of Fusion and Crystallization by Differential Scanning Calorimetry", (E 793 - 06), ASTM International, USA, 2006.

[65] R. German, "Powder Metallurgy Science", Second Edition, Metal Powder Industries Federation Press, New Jersey, USA, 1994. 
[66] ASTM Standard Committee, "Standard Guide for Preparation of Metallographic Specimens", (E 1-01), ASTM International, USA, 2007.

[67] "The Science Behind Materials Preparation", Buehler Ltd., Lake Bluff, USA, 2004.

[68] ASM Handbook Committee, "Metallography and Microstructures", Vol. 9, ASM International, Cleveland, USA, 2004.

[69] J.W. Weeton and R.A. Signorelli, "Effect of Heat Treatment upon Microstructures, Microconstituents, and Hardness of a Wrought Cobalt Base Alloy", Trans. Am. Soc. Met., Vol. 47, 1955, pp. 815-52.

[70] Y. Ning, "Effects of Sintering Process and the Coating of the Reinforcements on the Microstructure and Performance of Co-Based Superalloy Composites", M.A.Sc, Department of Mechanical and Aerospace Engineering of Carleton University, Ottawa, Canada, 2004.

[71] ASM Handbook Committee, "Corrosion: Fundamentals, Testing and Protection", Vol. 13.A, ASM International, Cleveland, USA, 2003.

[72] ASTM Standard Committee, "Standard Terminology Relating to Methods of Mechanical Testing", (E 6-09a), ASTM International, USA, 2009. 
[73] ASM Handbook Committee, "Mechanical Testing and Evaluation", Vol. 8, ASM International, USA, Cleveland, 2000.

[74] ASM Handbook Committee, "Friction, Lubrication, and Wear Technology", Vol. 18, ASM International, Cleveland, USA, 1992.

[75] M. Genest, "Image Processing for Automated Flaw Detection in Pulsed Thermography", 6th International Workshop-NDT Signal Processing, London, Canada, August 24-27, 2009.

[76] ASM Technical Book Committee, "Elements of Metallurgy and Engineering Alloys", ASM International, Cleveland, USA, 2008.

[77] A. Bakkar and V. Neubert, "Corrosion Behaviour of Carbon Fibres/Magnesium Metal Matrix Composite and Electrochemical Response of its Constituents", Electrochimica Acta, Vol. 54, 2009, pp. 1597-606.

[78] R. Liu and D.Y. Li, "A Finite Element Model Study on Wear Resistance of Pesudoelastic TiNi Alloy", Materials Science and Engineering A, Vol. 277, 2000, pp. 169-175. 
[79] R. Liu R, M.X. Yao, X. Wu, and P.C. Patnaik, "An Improved Wear-Resistant PTA Hardfacing: VWC/Stellite 21" Journal of Composite Materials, Vol. 40, 2006, pp. 2203-15.

[80] A. Khoddamzadeh, R. Liu, M. Liang, and Q. Yang, "Novel Wear-Resistant Materials - Carbon Fibre Reinforced Low-Carbon Stellite Alloy Composites", Composites: Part A, Vol. 43, 2012, pp. 344-352.

[81] D. C. Zipperian, "Metallographic Specimen Preparation and Hardness Testing Basics", Pace Technologies, Tucson, USA, 2007. 


\section{Tables}


Table 2.1 Physical properties of elemental cobalt $[1,2,21]$

\begin{tabular}{|c|c|c|}
\hline Property & Unit & Nominal Value \\
\hline Density $^{(a)}$ & $\begin{array}{c}\mathrm{g} / \mathrm{cc} \\
\left(\mathrm{b} / \mathrm{in}^{3}\right)\end{array}$ & $\begin{array}{c}8.85 \\
(0.319)\end{array}$ \\
\hline Melting Point & $\begin{array}{c}{ }^{\circ} \mathrm{C} \\
\left({ }^{\circ} \mathrm{F}\right)\end{array}$ & $\begin{array}{c}1493 \\
(2719)\end{array}$ \\
\hline Boiling Point & $\begin{array}{l}{ }^{\circ} \mathrm{C} \\
\left({ }^{\circ} \mathrm{F}\right)\end{array}$ & $\begin{array}{c}3100 \\
(5612)\end{array}$ \\
\hline Thermal Conductivity ${ }^{(a)}$ & $\mathrm{W} / \mathrm{m} \cdot \mathrm{K}$ & 69.04 \\
\hline Coefficient of Thermal Expansion ${ }^{(a)}$ & $\mu \mathrm{m} / \mathrm{m} . \mathrm{K}$ & 13.8 \\
\hline Specific Heat ${ }^{(a)}$ & $\mathrm{kJ} / \mathrm{kg} \cdot \mathrm{K}$ & 0.414 \\
\hline Electrical Conductivity ${ }^{(a)}$ & $\%$ IACS & 27.6 \\
\hline
\end{tabular}

Note: (a) At $20^{\circ} \mathrm{C}$

Table 2.2 Mechanical properties of elemental cobalt $[1,2,21]$

\begin{tabular}{|c|c|c|}
\hline Property & Unit & Nominal Value \\
\hline Hardness $^{(\mathrm{a})}$ & $\mathrm{HV}$ & $140-250$ \\
\hline Tensile Strength $^{(\mathrm{b})}$ & $\begin{array}{c}\mathrm{MPa} \\
(\mathrm{ksi})\end{array}$ & $\begin{array}{c}800-875 \\
(116-127)\end{array}$ \\
\hline Elongation $^{(\mathrm{b})}$ & $\%$ & $15-30$ \\
\hline $0.2 \%$ yield strength $^{(\mathrm{b})}$ & $\mathrm{MPa}$ & $\begin{array}{c}305-345 \\
(44-50)\end{array}$ \\
\hline Young's Modulus $^{(\mathrm{ksi})}$ & $\begin{array}{c}\mathrm{GPa} \\
(\mathrm{psi})\end{array}$ & $\begin{array}{c}211 \\
\left(30.6 \times 10^{6}\right)\end{array}$ \\
\hline Shear Modulus $^{8}$ & $\mathrm{GPa}$ & $\begin{array}{c}82 \\
(\mathrm{psi})\end{array}$ \\
\hline Compressive Modulus $^{\left(11.9 \times 10^{6}\right)}$ \\
\hline
\end{tabular}

Note: (a) At $20^{\circ} \mathrm{C}$, (b) Annealed high purity (99.5\%) vacuum-melted cobalt 
Table 2.3 Chemical compositions (wt\%) of Tribaloy alloys

\begin{tabular}{|c|c|c|c|c|c|c|}
\hline $\begin{array}{c}\text { Tribaloy } \\
\text { alloy }\end{array}$ & Co & $\mathrm{Ni}$ & $\mathrm{Cr}$ & $\mathrm{Mo}$ & $\mathrm{Si}$ & $\begin{array}{c}\text { Laves phase content } \\
\text { (vol\%) }\end{array}$ \\
\hline T-400 & 62 & - & 8.5 & 28.5 & 2.6 & $\sim 45$ \\
\hline T-800 & 52 & - & 17 & 28 & 3 & $\sim 55$ \\
\hline T-700 & - & 50 & 15 & 32 & 3 & $\sim 60$ \\
\hline T-100 & 55 & - & - & 35 & 10 & $\sim 65$ \\
\hline
\end{tabular}

Table 2.4 Compositions (wt\%, Co in balance) of various Stellite alloys

\begin{tabular}{|c|c|c|c|c|c|c|c|c|c|c|}
\hline Alloy & Process & $\mathrm{Cr}$ & $\mathrm{W}$ & $\mathrm{Mo}$ & $\mathrm{C}$ & $\mathrm{Fe}$ & $\mathrm{Ni}$ & $\mathrm{Si}$ & $\mathrm{Mn}$ & Others \\
\hline Stellite 1 & Cast & 30 & 13 & 0.5 & 2.5 & 3 & 1.5 & 1.3 & 0.5 & \\
\hline Stellite 3 & P/M & 30 & 12.5 & & 2.4 & 5 & 3.5 & 2 & 2 & $1 \mathrm{~B}$ \\
\hline Stellite 4 & Cast & 30 & 14 & 1 & 0.57 & 3 & 3 & 2 & 1 & \\
\hline Stellite 6 & Cast & 29 & 4.5 & 1.5 & 1.2 & 3 & 3 & 1.5 & 1 & \\
\hline Stellite 6B & Wrought & 30 & 4 & 1.5 & 1 & 3 & 2.5 & 0.7 & 1.4 & \\
\hline Stellite 12 & Cast & 30 & 8.3 & & 1.4 & 3 & 1.5 & 0.7 & 2.5 & \\
\hline Stellite 21 & Cast & 27 & & 5.5 & 0.25 & 3 & 2.75 & 1 & 1 & \\
\hline Stellite 20 & Cast & 33 & 17.5 & & 2.45 & 2.5 & 2.5 & & 1 & \\
\hline Stellite 22 & Cast & 27 & & 11 & 0.25 & 3 & 2.75 & 1 & 1 & \\
\hline Stellite 31 & Cast & 22 & 7.5 & & 0.5 & 1.5 & 10 & 0.5 & 0.5 & \\
\hline Stellite 694 & Cast, P/M & 28 & 19 & & 1 & 5 & & 1 & 1 & \\
\hline Stellite 712 & Cast, P/M & 29 & & 8.5 & 2 & 3 & 3 & 1.5 & 1.5 & \\
\hline Stellite 720 & Cast & 33 & & 18 & 2.5 & 3 & 3 & 1.5 & 1.5 & $0.3 \mathrm{~B}$ \\
\hline Stellite 703 & Cast & 32 & & 12 & 2.4 & 3 & 3 & 1.5 & 1.5 & \\
\hline Stellite 706 & Cast & 29 & & 5 & 1.2 & 3 & 3 & 1.5 & 1.5 & \\
\hline Stellite 706K & Wrought & 29 & & 6 & 1.4 & 3 & 3 & 1.5 & 1.5 & \\
\hline Stellite 6B & Wrought & 30 & 4 & 1.5 & 1 & 3 & 2.5 & 0.7 & 1.4 & \\
\hline Stellite 98M2 & P/M & 30 & 18.5 & 0.8 & 2 & 5 & 3.5 & 1 & 1 & $4.2 \mathrm{~V}, 1 \mathrm{~B}$ \\
\hline
\end{tabular}


Table 3.1 Nominal chemical composition (wt\%) of Stellite 25 [58]

\begin{tabular}{|c|c|c|c|c|c|c|c|c|}
\hline Alloy & $\mathrm{Co}$ & $\mathrm{Cr}$ & $\mathrm{W}$ & $\mathrm{C}$ & $\mathrm{Fe}$ & $\mathrm{Ni}$ & $\mathrm{Si}$ & $\mathrm{Mn} / \mathrm{Mo}$ \\
\hline $\begin{array}{c}\text { Stellite } \\
25\end{array}$ & Bal. & 20 & 15 & 0.1 & $\begin{array}{c}3 \\
(\max )\end{array}$ & 10 & 1 & 1.5 \\
\hline
\end{tabular}

Table 3.2 Physical properties of Stellite 25 [58]

\begin{tabular}{|c|c|c|}
\hline Property & Unit & Nominal Value \\
\hline Density $^{(\mathrm{a})}$ & $\begin{array}{c}\mathrm{g} / \mathrm{cc} \\
(\mathrm{lb} / \mathrm{in} .\end{array}$ & $\begin{array}{c}8.31 \\
(0.300)\end{array}$ \\
\hline Melting Range & $\begin{array}{c}{ }^{3} \mathrm{C} \\
\left({ }^{\circ} \mathrm{F}\right)\end{array}$ & $\begin{array}{c}1329-1410 \\
(2425-2573)\end{array}$ \\
\hline Thermal Conductivity $^{(\mathrm{a})}$ & $\mathrm{W} / \mathrm{m} \cdot \mathrm{K}$ & $\begin{array}{c}9.37^{(\mathrm{a})} \\
11.83^{(\mathrm{b})}\end{array}$ \\
\hline Coefficient of Thermal Expansion $^{(\mathrm{c})}$ & $\mu \mathrm{m} / \mathrm{m} \cdot \mathrm{K}$ & 12.24 \\
\hline
\end{tabular}

Note: (a) At $20^{\circ} \mathrm{C}$, (b) At $149^{\circ} \mathrm{C}$, and (c) At $93^{\circ} \mathrm{C}$

Table 3.3 Mechanical properties of Stellite 25 at room temperature [58]

\begin{tabular}{|c|c|c|}
\hline Property & Unit & Nominal Value \\
\hline Hardness & HRC & 24 \\
\hline Tensile Strength & $\begin{array}{c}\mathrm{MPa} \\
(\mathrm{ksi})\end{array}$ & $\begin{array}{c}925 \\
(134)\end{array}$ \\
\hline Elongation & $\mathrm{A} \%$ & 5 \\
\hline $0.2 \%$ yield strength & $\mathrm{MPa}$ & 895 \\
$(\mathrm{ksi})$ & $(130)$ \\
\hline Young's Modulus & $\begin{array}{c}\text { GPa } \\
(\mathrm{psi})\end{array}$ & $\begin{array}{c}207 \\
\left(30.0 \times 10^{6}\right)\end{array}$ \\
\hline
\end{tabular}


Table 3.4 Physical properties of chopped plain carbon fibre at room temperature [60]

\begin{tabular}{|c|c|c|}
\hline Property & Unit & Nominal Value \\
\hline Density & $\begin{array}{c}\mathrm{g} / \mathrm{cc}^{3} \\
\left(\mathrm{lb} / \mathrm{in}^{3}\right)\end{array}$ & $\begin{array}{c}1.8 \\
(0.065)\end{array}$ \\
\hline Fibre Length & $\begin{array}{c}\mathrm{mm} \\
\text { (in.) }\end{array}$ & $\begin{array}{c}3 \\
(1 / 8)\end{array}$ \\
\hline Filament Diameter & $\begin{array}{c}\mu \mathrm{m} \\
\text { (in.) }\end{array}$ & $\begin{array}{c}6 \\
\left(0.24 \times 10^{-3}\right)\end{array}$ \\
\hline Cross-Sectional Area/Filament & $\begin{array}{c}\mu \mathrm{m} \\
\text { (in.) }\end{array}$ & $\begin{array}{c}3.3 \times 10^{-5} \\
\left(4.7 \times 10^{-8}\right)\end{array}$ \\
\hline Moisture Content & $\%$ & 0.3 \\
\hline Electrical Resistivity & $\mu \Omega /$ inch & 657 \\
\hline
\end{tabular}

Table 3.5 Mechanical properties of chopped plain CF at room temperature [60]

\begin{tabular}{|c|c|c|}
\hline Property & Unit & Nominal Value \\
\hline Tensile Strength & $\begin{array}{c}\mathrm{MPa} \\
(\mathrm{ksi})\end{array}$ & $\begin{array}{c}>3450 \\
(>500)\end{array}$ \\
\hline Elongation & $\%$ & 1.7 \\
\hline Young's Modulus & $\begin{array}{c}\mathrm{GPa} \\
(\mathrm{psi})\end{array}$ & $\begin{array}{c}>207 \\
\left(30.0 \times 10^{6}\right)\end{array}$ \\
\hline
\end{tabular}

Table 3.6 Physical properties of chopped nickel-coated CF at room temperature [60]

\begin{tabular}{|c|c|c|}
\hline Property & Unit & Nominal Value \\
\hline Density without Size & $\begin{array}{c}\mathrm{g} / \mathrm{cc} \\
\left(\mathrm{lb} / \mathrm{in}^{3}\right)\end{array}$ & $\begin{array}{c}2.7 \\
(0.098)\end{array}$ \\
\hline Bulk Density & $\begin{array}{c}\mathrm{g} / \mathrm{cc} \\
\left(\mathrm{lb} / \mathrm{in}^{3}\right)\end{array}$ & $\begin{array}{c}0.4 \\
(0.014)\end{array}$ \\
\hline Fibre Length & $\begin{array}{c}\mathrm{mm} \\
(\mathrm{in})\end{array}$ & $\begin{array}{c}6 \pm 1.5 \\
(0.24 \pm 0.06)\end{array}$ \\
\hline Filament Diameter + Nickel Layer & $\begin{array}{c}\mu \mathrm{m} \\
(\mathrm{in})\end{array}$ & $\begin{array}{c}7.5+0.25 \\
\left((295+9) \times 10^{-6}\right)\end{array}$ \\
\hline Thickness of Nickel Layer & $\begin{array}{c}\mu \mathrm{m} \\
(\mathrm{in})\end{array}$ & $\begin{array}{c}0.25 \\
\left(9.8 \times 10^{-6}\right)\end{array}$ \\
\hline Electrical Resistivity & $\mu \Omega / \mathrm{inch}$ & 3.94 \\
\hline
\end{tabular}


Table 3.7 Mechanical properties of chopped nickel-coated CF at room temperature [60]

\begin{tabular}{|c|c|c|}
\hline Property & Unit & Nominal Value \\
\hline Tensile Strength & $\begin{array}{c}\mathrm{MPa} \\
(\mathrm{ksi})\end{array}$ & $\begin{array}{c}2550 \\
(370)\end{array}$ \\
\hline Elongation & $\%$ & 1.7 \\
\hline Young's Modulus & $\begin{array}{c}\mathrm{GPa} \\
(\mathrm{psi})\end{array}$ & $\begin{array}{c}238 \\
\left(34.5 \times 10^{6}\right)\end{array}$ \\
\hline
\end{tabular}

Table 3.8 Chemical compositions of Stellite 25 and Stellite 25-based composites

\begin{tabular}{|c|c|c|c|}
\hline Designation & $\begin{array}{c}\text { Stellite 25 } \\
\text { wt\% (vol.\%) }\end{array}$ & $\begin{array}{c}\text { Chopped CF } \\
\text { wt\% (vol.\%) }\end{array}$ & $\begin{array}{c}\text { Ni-Coated CF } \\
\text { wt\% (vol.\%) }\end{array}$ \\
\hline A & $100(100)$ & $0(0)$ & 0 \\
\hline B & $99.75(98.8)$ & $0.25(1.2)$ & 0 \\
\hline C & $99.5(97.7)$ & $0.5(2.3)$ & 0 \\
\hline D & $99(95.5)$ & $1(4.5)$ & 0 \\
\hline E & $98(91.4)$ & $2(8.6)$ & 0 \\
\hline F & $95(80.4)$ & $5(19.6)$ & 0 \\
\hline K & $99.75(99.2)$ & 0 & $0.25(0.8)$ \\
\hline L & $99.5(98.4)$ & 0 & $0.5(1.6)$ \\
\hline M & $99(97)$ & 0 & $1(3)$ \\
\hline N & $98(94.1)$ & 0 & $2(5.9)$ \\
\hline O & $95(86.1)$ & 0 & $5(13.9)$ \\
\hline P & $90(74.5)$ & 0 & $10(25.5)$ \\
\hline
\end{tabular}


Table 4.1 General properties of phenolics $[66,81]$

\begin{tabular}{|c|c|c|}
\hline Property & Unit & Quality / Nominal Value \\
\hline Resin Type & - & Thermoset \\
\hline Form & - & Granular \\
\hline Colours & - & Black, Red, Green \\
\hline Clarity & - & None \\
\hline Cost & - & Low \\
\hline Ease of Use & - & Excellent \\
\hline Cycle Times & - & Excellent \\
\hline Edge Retention & - & Fair \\
\hline Specific Gravity & $\begin{array}{c}\mathrm{g} / \mathrm{cc}^{3} \\
\left(\mathrm{lb} / \mathrm{in}^{3}\right)\end{array}$ & $\begin{array}{c}1.4 \\
(0.050)\end{array}$ \\
\hline Hardness & - & Low \\
\hline
\end{tabular}


Table 4.2 The procedure of grinding and polishing operations [66-68]

\begin{tabular}{|c|c|c|c|c|}
\hline Surface & Abrasive/Size & $\begin{array}{c}\text { Load N (Lb.)/ } \\
\text { Specimen }\end{array}$ & $\begin{array}{c}\text { Base } \\
\text { Speed(rpm)/ } \\
\text { Direction }\end{array}$ & $\begin{array}{c}\text { Time } \\
\text { (min:sec) }\end{array}$ \\
\hline $\begin{array}{c}\text { CarbiMet }{ }^{\circledR} \\
\text { Abrasive Grinding } \\
\text { Discs }\end{array}$ & $\begin{array}{c}320-(\mathrm{P400}) \\
\text { Grit SiC } \\
\text { Water Cooled }\end{array}$ & $27(6)$ & $250 /$ Contra $^{3}$ & $\begin{array}{l}\text { Until } \\
\text { Plane }\end{array}$ \\
\hline $\begin{array}{c}\text { BuehlerHercules }^{\mathrm{TM}} \\
\text { S } \\
\text { Rigid Grinding } \\
\text { Disc }\end{array}$ & $\begin{array}{l}9 \mu \mathrm{m} \text { MetaDi@ } \\
\text { Supreme } \\
\text { Diamond } \\
\text { Suspension }\end{array}$ & $27(6)$ & $100 /$ Comp. $^{b}$ & $5: 00$ \\
\hline $\begin{array}{l}\text { TexMet@ } 1000 \\
\text { Polishing Pad }\end{array}$ & $\begin{array}{l}3 \mu \mathrm{m} \text { MetaDi® } \\
\text { Supreme } \\
\text { Diamond } \\
\text { Suspension }\end{array}$ & $27(6)$ & $100 /$ Contra $^{a}$ & $5: 00$ \\
\hline $\begin{array}{l}\text { TexMet@ } 1000 \\
\text { Polishing Pad }\end{array}$ & $\begin{array}{l}\text { l } \mu \mathrm{m} \text { MetaDiß } \\
\text { Supreme } \\
\text { Diamond } \\
\text { Suspension }\end{array}$ & $27(6)$ & $100 /$ Contra $^{a}$ & $3: 00$ \\
\hline $\begin{array}{c}\text { Microcloth } \\
\text { Polishing Pad }\end{array}$ & $\begin{array}{c}0.05 \mu \mathrm{m} \\
\text { MasterPrep® } \\
\text { Alumina } \\
\text { Suspension }\end{array}$ & $27(6)$ & $100 /$ Contra $^{a}$ & 3:00 \\
\hline
\end{tabular}

Note:

(a) Contra $=$ platen and specimen holder rotate in opposite directions

(b) Comp. = Complementary (platen and specimen holder both rotate in the same direction)

Table 4.3 Carbide identifying reagent and etching procedure $[1,68,69]$

\begin{tabular}{|c|c|c|c|}
\hline \multicolumn{2}{|c|}{ First Etchant } & \multicolumn{2}{c|}{ Staining Method } \\
\hline Solution & Method & Solution & Method \\
& & & \\
& & 1 Part $(20 \%$ Potassium & \\
$2 \%$ Chromic & & Permanganate $\left(\mathrm{KMnO}_{4}\right)+$ & \\
Acid $\left(\mathrm{H}_{2} \mathrm{CrO}_{4}\right)$ & Light & $80 \%$ Water $\left.\left(\mathrm{H}_{2} \mathrm{O}\right)\right)+$ & Immerse 7 s at \\
+ & Electrolytic & 1 Part $(8 \%$ Sodium & Room Temperature \\
$98 \%$ Water & Etch & Hydroxide & \\
$\left(\mathrm{H}_{2} \mathrm{O}\right)$ & & $(\mathrm{NaOH})+92 \%$ Water & \\
& & $\left(\mathrm{H}_{2} \mathrm{O}\right)$ & \\
& & & \\
& & & \\
\hline
\end{tabular}


Table 5.1 Carbide volume fraction in plain CF reinforced composite specimens

\begin{tabular}{|c|c|c|}
\hline & $\begin{array}{c}\text { Carbide } \\
\text { Draction } \\
\text { (vol.\%) }\end{array}$ & $\begin{array}{c}\text { Standard } \\
\text { Deviation }\end{array}$ \\
\hline A & 3.8 & 0.1 \\
\hline B & 4.2 & 0.1 \\
\hline C & 4.8 & 0.3 \\
\hline D & 5.2 & 0.2 \\
\hline E & 5.8 & 0.2 \\
\hline F & 6.4 & 0.4 \\
\hline
\end{tabular}

Table 5.2 Specific densities of the plain CF reinforced composite specimens

\begin{tabular}{|c|c|}
\hline Designation & $\begin{array}{c}\text { Specific Density } \\
\mathrm{g} / \mathrm{cm}^{3}\left(\mathrm{lb} / \mathrm{in}^{3}\right)\end{array}$ \\
\hline A & $9.823(0.3549)$ \\
\hline B & $8.769(0.3175)$ \\
\hline C & $8.751(0.3161)$ \\
\hline D & $8.716(0.3149)$ \\
\hline E & $8.647(0.3124)$ \\
\hline F & $8.437(0.3048)$ \\
\hline
\end{tabular}


Table 5.3 Carbide volume fraction in Ni-coated CF reinforced composite specimens

\begin{tabular}{|c|c|}
\hline Designation & $\begin{array}{c}\text { Carbide } \\
\text { Fraction } \\
\text { (vol.\%) }\end{array}$ \\
\hline $\mathrm{A}$ & 3.8 \\
\hline $\mathrm{K}$ & 3.9 \\
\hline $\mathrm{L}$ & 3.8 \\
\hline $\mathrm{M}$ & 3.9 \\
\hline $\mathrm{N}$ & 3.9 \\
\hline $\mathrm{O}$ & 3.7 \\
\hline $\mathrm{P}$ & 3.8 \\
\hline
\end{tabular}

Table 5.4 Specific densities of Ni-coated CF reinforced composite specimens

\begin{tabular}{|c|c|}
\hline Designation & $\begin{array}{c}\text { Specific density } \\
\mathrm{g} / \mathrm{cm}^{3}\left(\mathrm{lb} / \mathrm{in}{ }^{3}\right)\end{array}$ \\
\hline $\mathrm{A}$ & $9.823(0.3549)$ \\
\hline $\mathrm{K}$ & $9.744(0.3520)$ \\
\hline $\mathrm{L}$ & $9.709(0.3507)$ \\
\hline $\mathrm{M}$ & $9.609(0.3471)$ \\
\hline $\mathrm{N}$ & $9.403(0.3397)$ \\
\hline $\mathrm{O}$ & $8.833(0.3191)$ \\
\hline $\mathrm{P}$ & $8.001(0.2890)$ \\
\hline
\end{tabular}


Table 5.5 Mean microhardness values of specimen $F$, specimen $\mathrm{O}$, and their carbidic phase.

\begin{tabular}{|c|c|c|}
\hline Indentation & HRC (HV) & $\begin{array}{c}\text { Standard } \\
\text { Deviation }\end{array}$ \\
\hline $\mathrm{F}_{1}$ & $482(48)$ & 22 \\
\hline $\mathrm{F}_{2}$ & $520(50)$ & 16 \\
\hline $\mathrm{F}_{3}$ & $580(54)$ & 19 \\
\hline $\mathrm{F}_{4}$ & $566(53)$ & 25 \\
\hline $\mathrm{O}_{1}$ & $434(44)$ & 13 \\
\hline $\mathrm{O}_{2}$ & $483(48)$ & 26 \\
\hline $\mathrm{O}_{3}$ & $494(49)$ & 39 \\
\hline $\mathrm{O}_{4}$ & $518(50)$ & 7 \\
\hline Carbide & $1510(-)$ & 45 \\
\hline
\end{tabular}

Table 5.6 Relative thermal properties obtained with pulsed thermography technique

\begin{tabular}{|c|c|c|c|}
\hline Specimen & $\begin{array}{c}\Delta \mathrm{T} \text { Normalized } \\
\left({ }^{\circ} \mathrm{C}\right)\end{array}$ & $\begin{array}{c}\mathrm{C} \times \mathrm{K}^{(\mathrm{a})} \\
\left(\mathrm{J} . \mathrm{W} \cdot \mathrm{m}^{-1} \cdot \mathrm{k}^{-1}\right)\end{array}$ & $\begin{array}{c}\mathrm{C} \times \mathrm{K}^{(\mathrm{b})} \\
\left(\mathrm{J} . \mathrm{W} \mathrm{m}^{-1} \cdot \mathrm{k}^{-1}\right)\end{array}$ \\
\hline $\mathrm{A}$ & 1.198 & 1410 & 173.2 \\
\hline $\mathrm{F}$ & 1.184 & 1682 & 206.5 \\
\hline $\mathrm{O}$ & 1.145 & 1717 & 210.9 \\
\hline
\end{tabular}

Note: (a) At $t=1 \mathrm{~s}$, and (b) At $t=8.15 \mathrm{~s}$ (last frame in the acquisition)

Table 6.1 Property comparison between the developed composites and PTFE based sliding bearing materials

\begin{tabular}{|c|c|c|c|}
\hline Designation & $\begin{array}{c}\text { Corrosion Rate } \\
(\text { mils/yr) }\end{array}$ & $\begin{array}{c}\text { Friction } \\
\text { Coefficient }^{(\mathrm{a})}\end{array}$ & $\begin{array}{c}\text { Specific Wear Rate } \\
\left(10^{-5} \mathrm{~mm}^{3} / \mathrm{N} . \mathrm{m}\right)\end{array}$ \\
\hline $\mathrm{F}$ & 192.57 & 0.15 & 0.86 \\
\hline $\mathrm{O}$ & 269.19 & 0.15 & 1.89 \\
\hline $\mathrm{Z}^{(\mathrm{b})}$ & 310.42 & 0.159 & 2.6 \\
\hline
\end{tabular}

Note: (a) The constant value after 55 min sliding, and (b) $40 \mathrm{wt} \%$ PTFE, $45 \mathrm{wt} \%$ bronze and $15 \mathrm{wt} \% \mathrm{~T}-401$ 


\section{Figures}




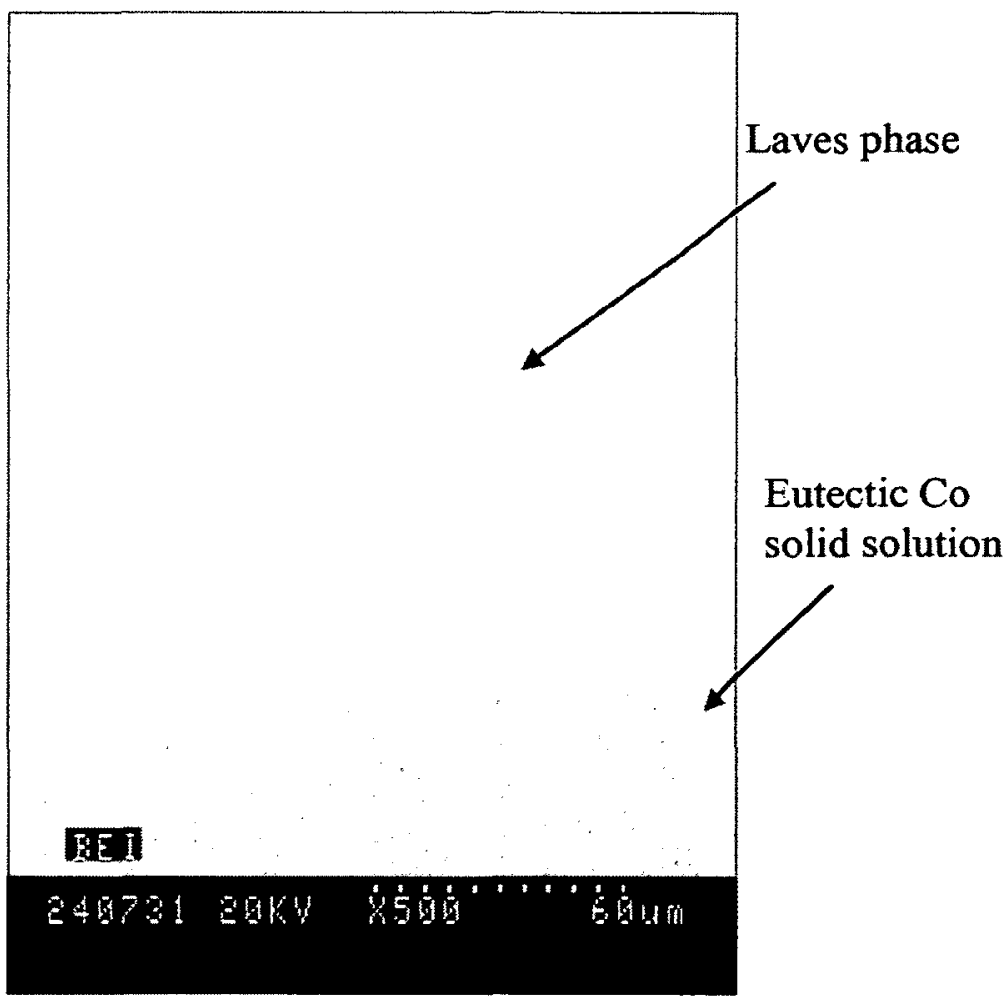

(a)

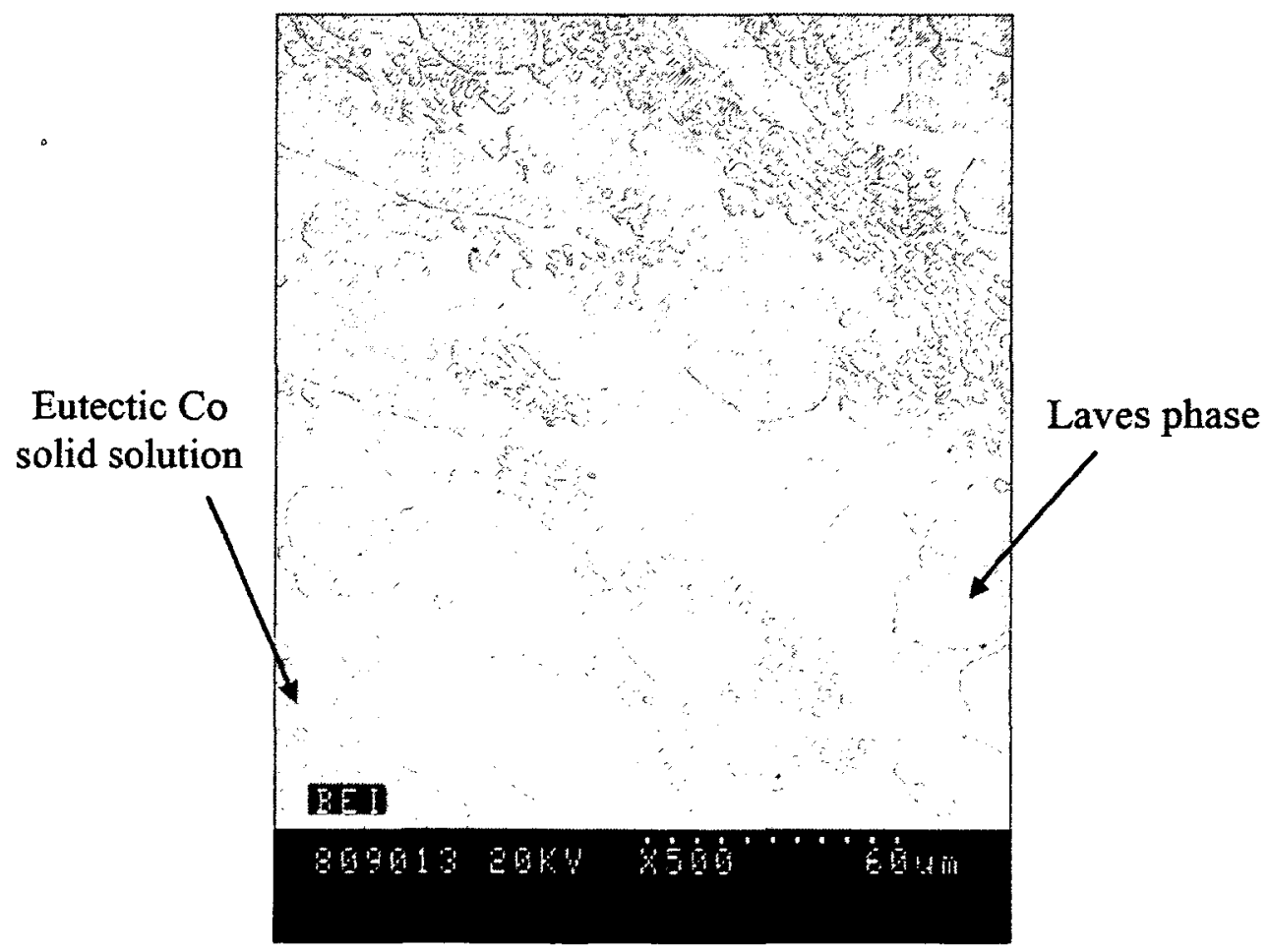

(b)

Fig. 2.1. Typical microstructure of Tribaloy alloys: (a) T-400 and (b) T-800. 


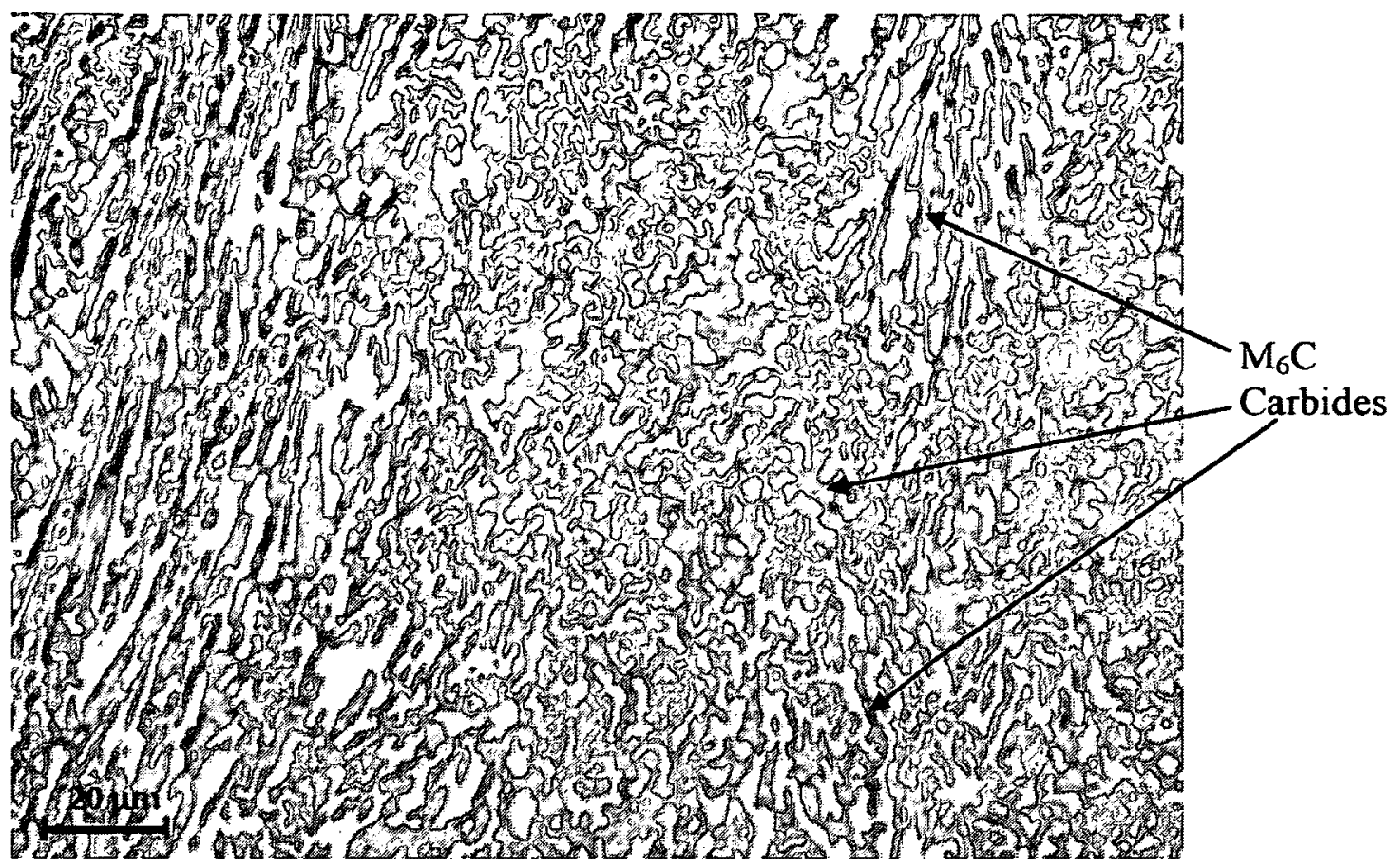

(a)

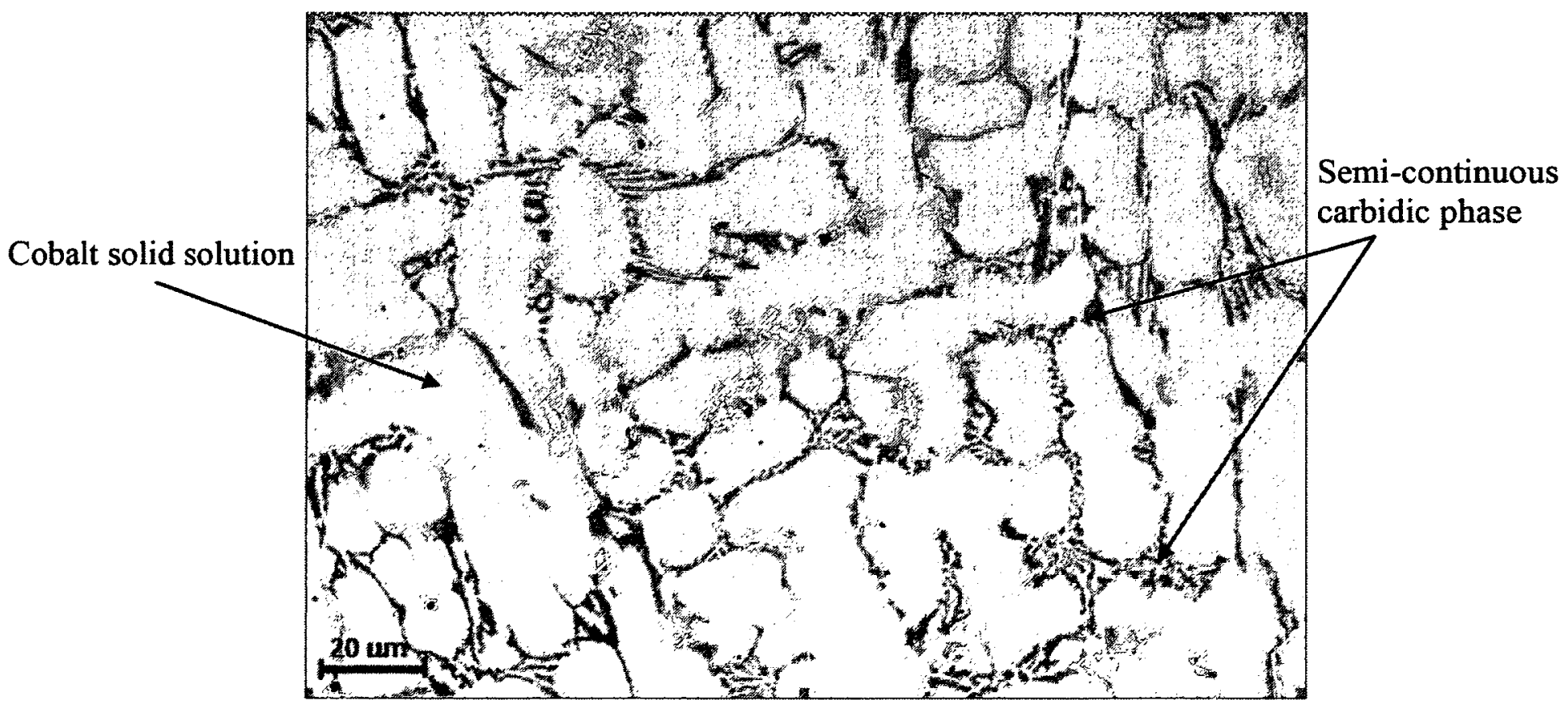

(b) 


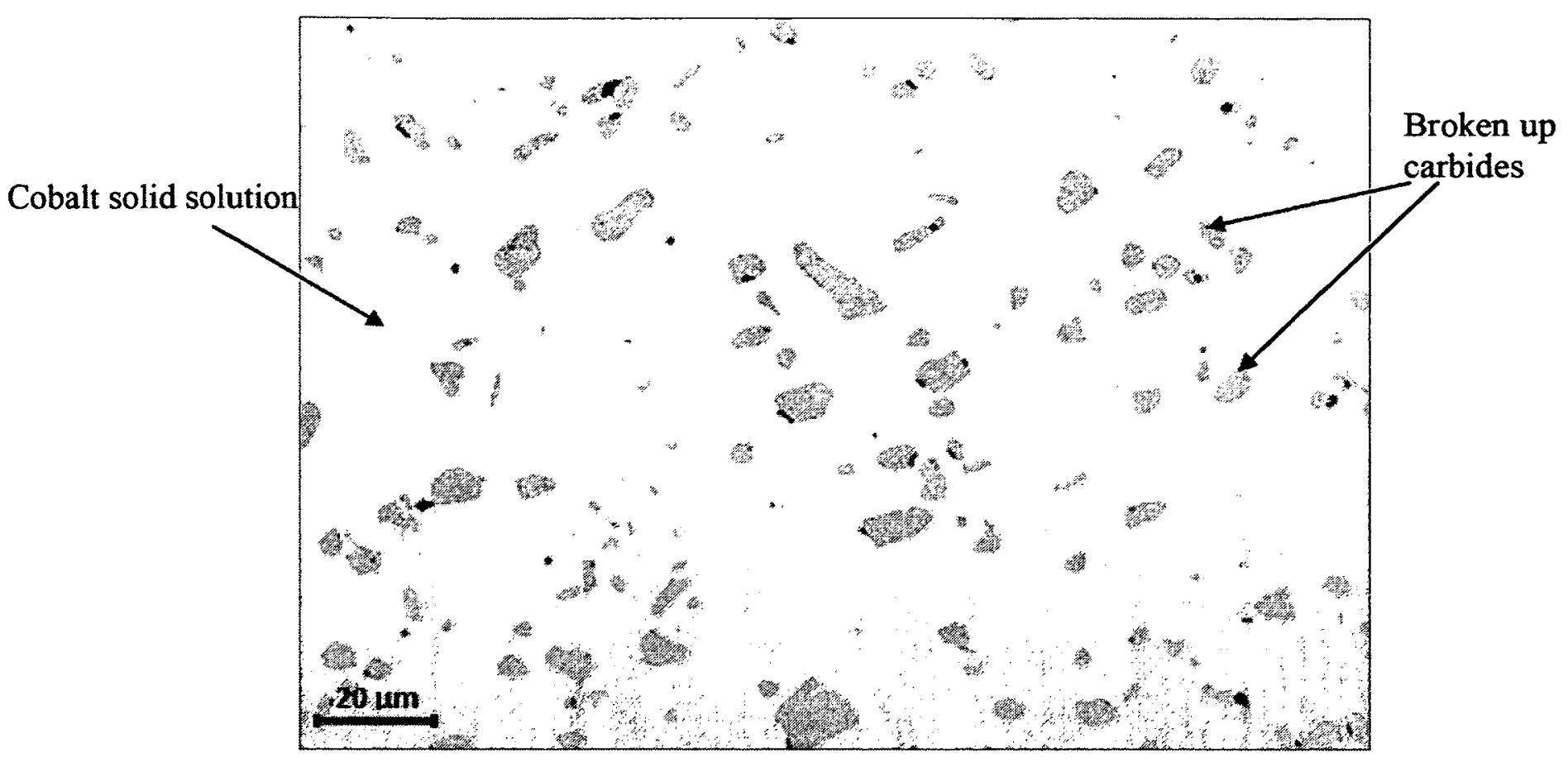

(c)

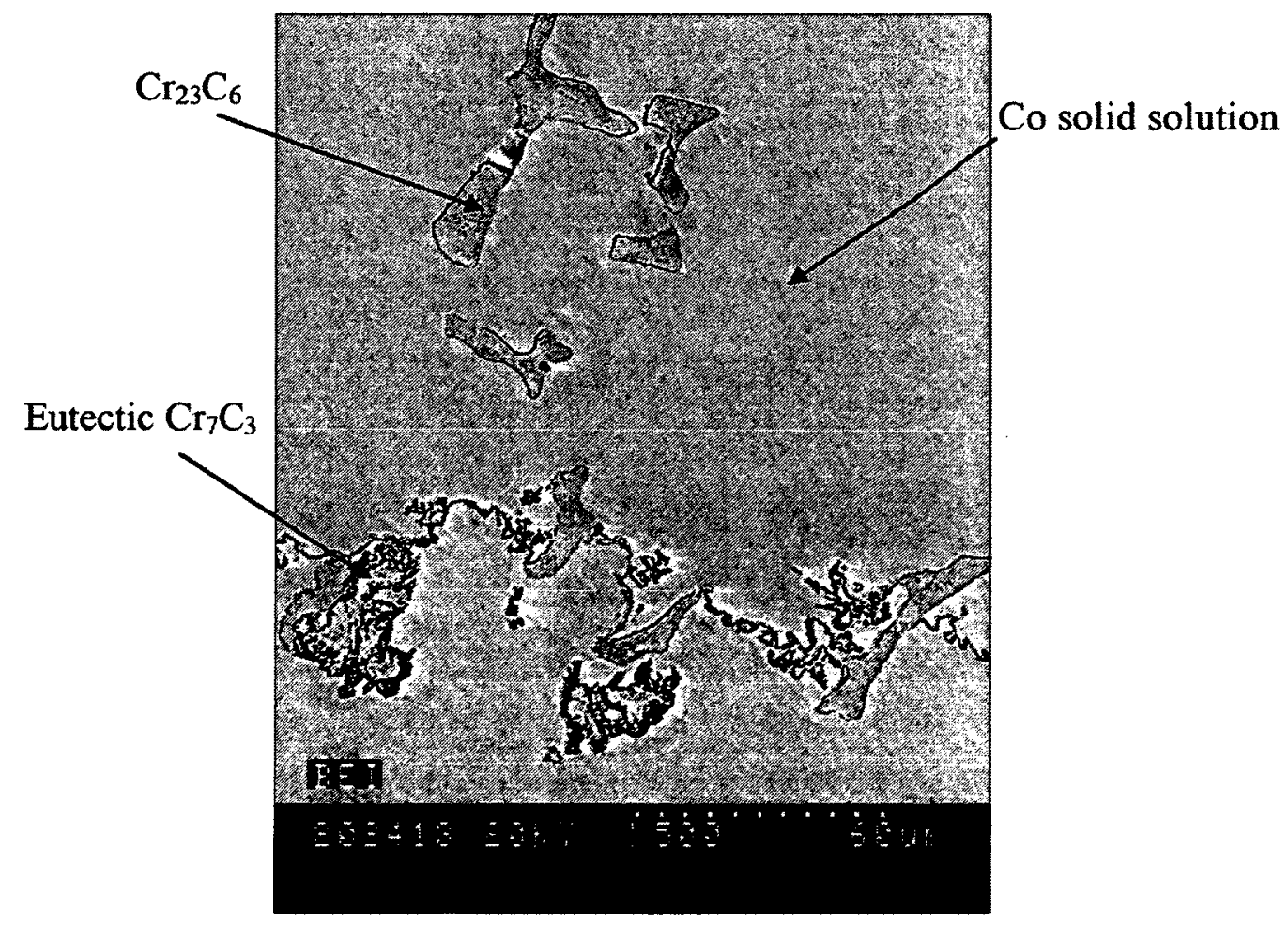

(d)

Fig. 2.2. Typical microstructure of Stellite alloys: (a) Stellite 1, (b) Stellite 6 (cast), (c) Stellite 6B (wrought), and (d) Stellite 21 [70] 


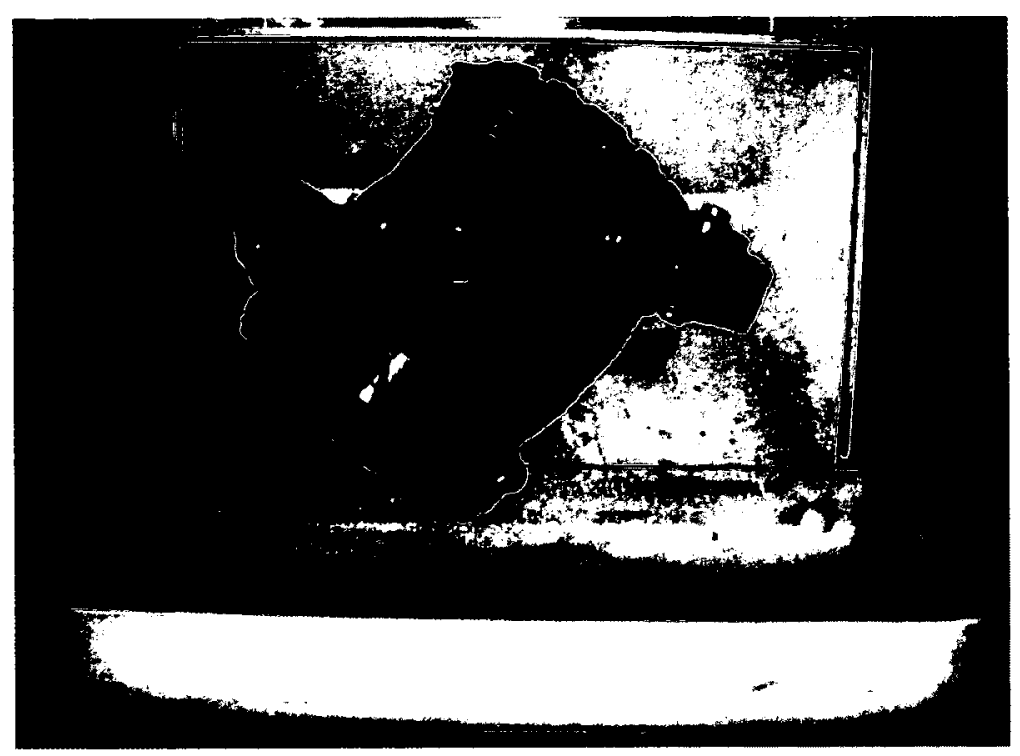

Fig. 3.1. Three dimensional Turbula type T2C mixer.

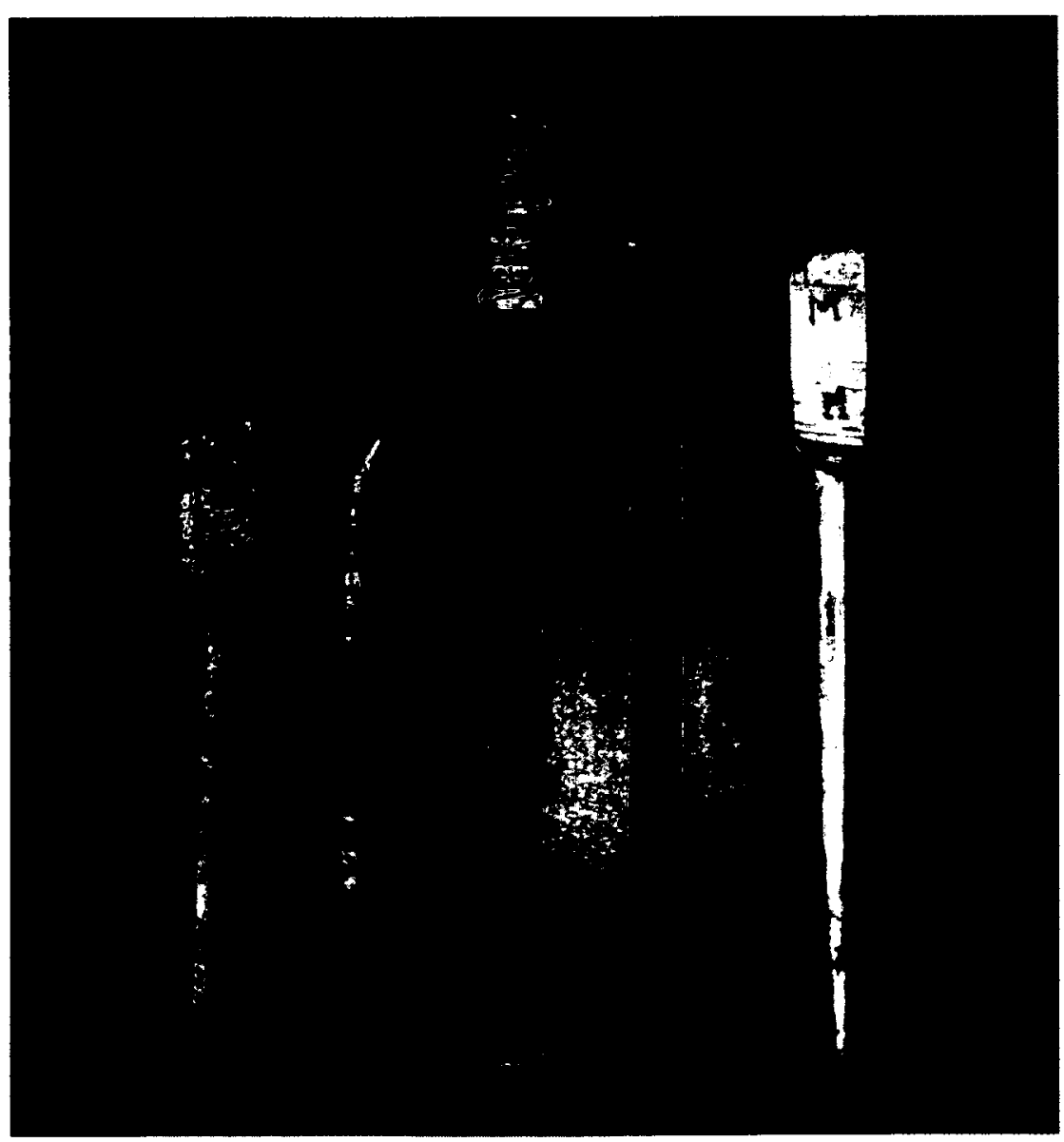

Fig. 3.2. Consolidation container specimens. 


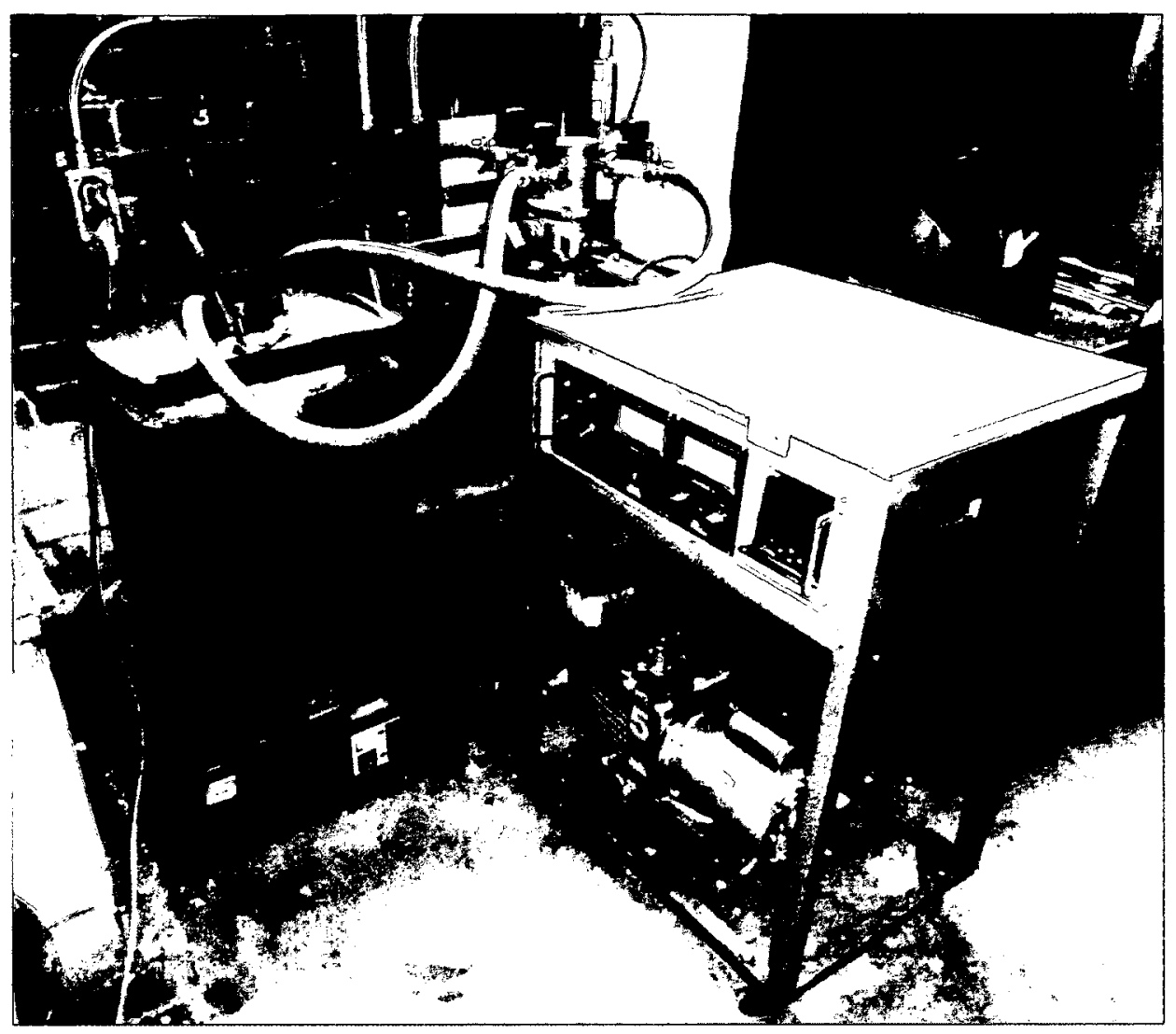

Fig. 3.3. Vacuum outgassing pump.

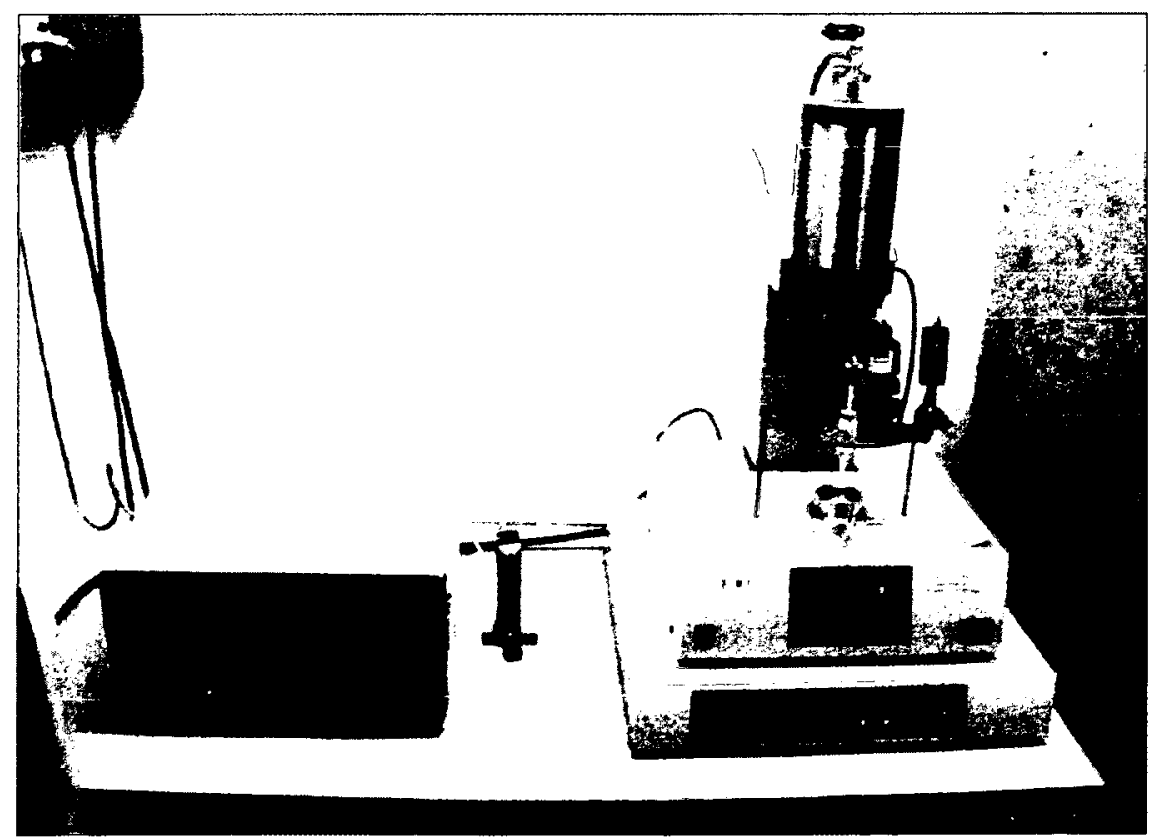

Fig. 3.4. Netzsch $404 C$ Pegasus heat flux DSC instrument. 


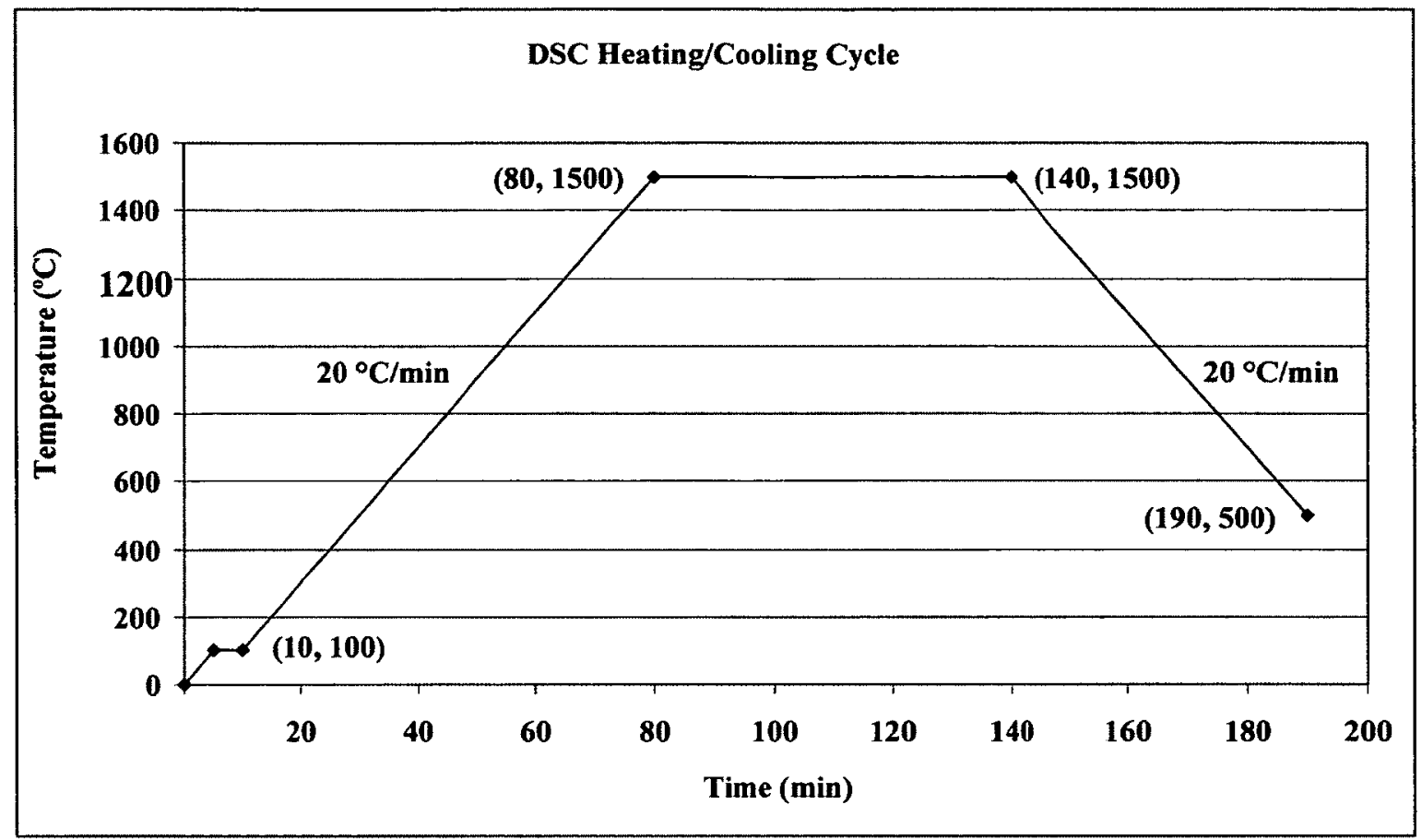

Fig. 3.5. DSC heating/cooling cycle.

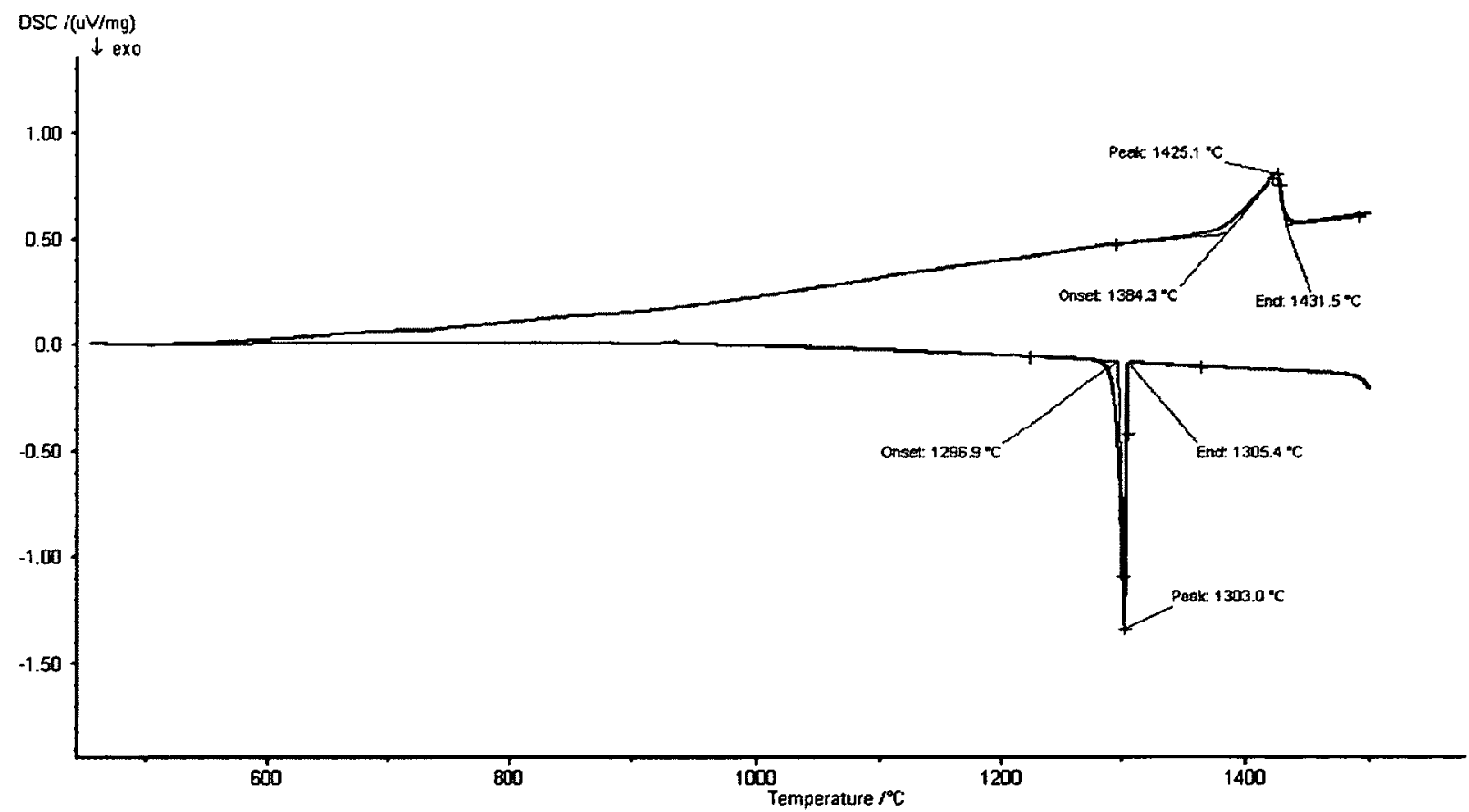

(a) 


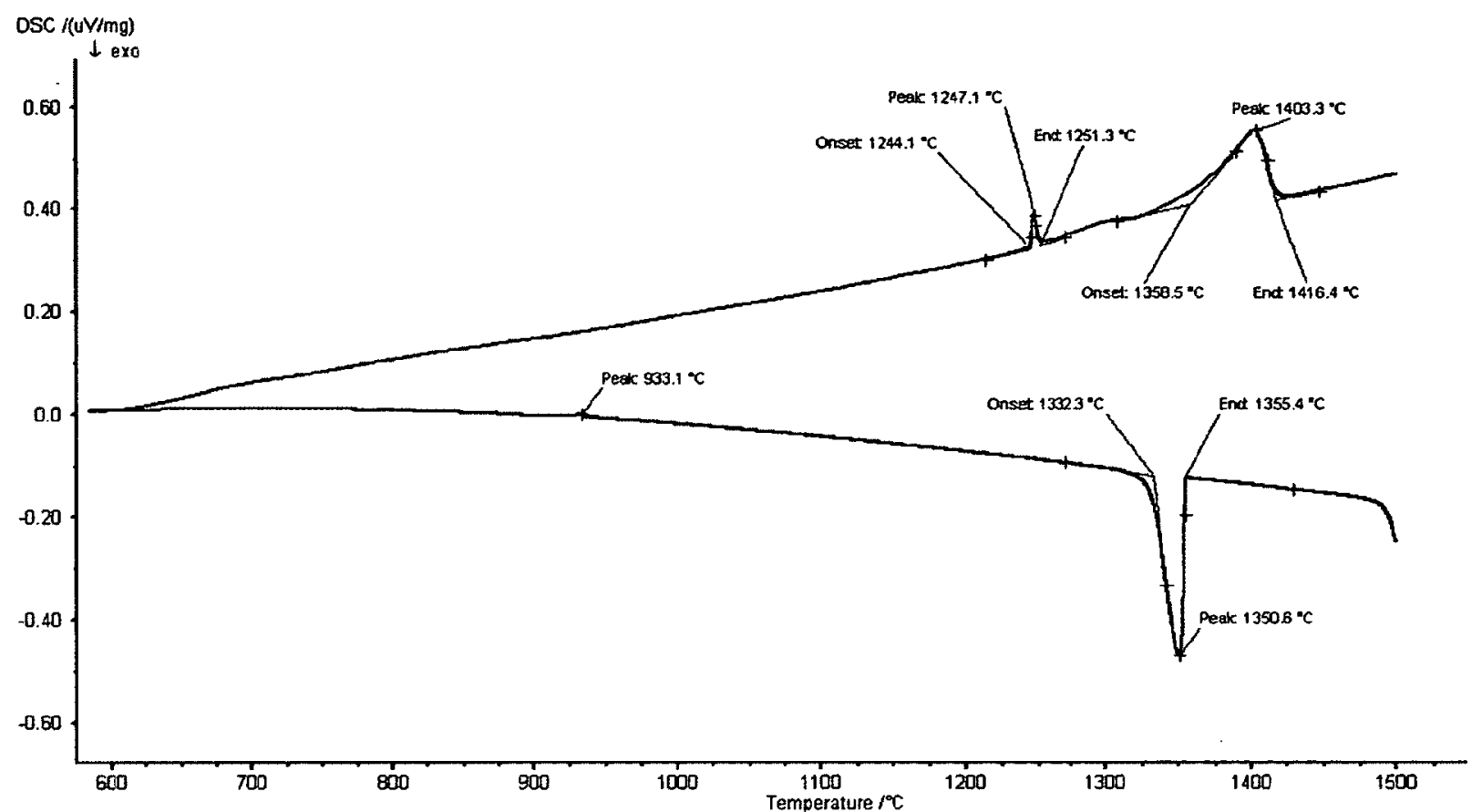

(b)

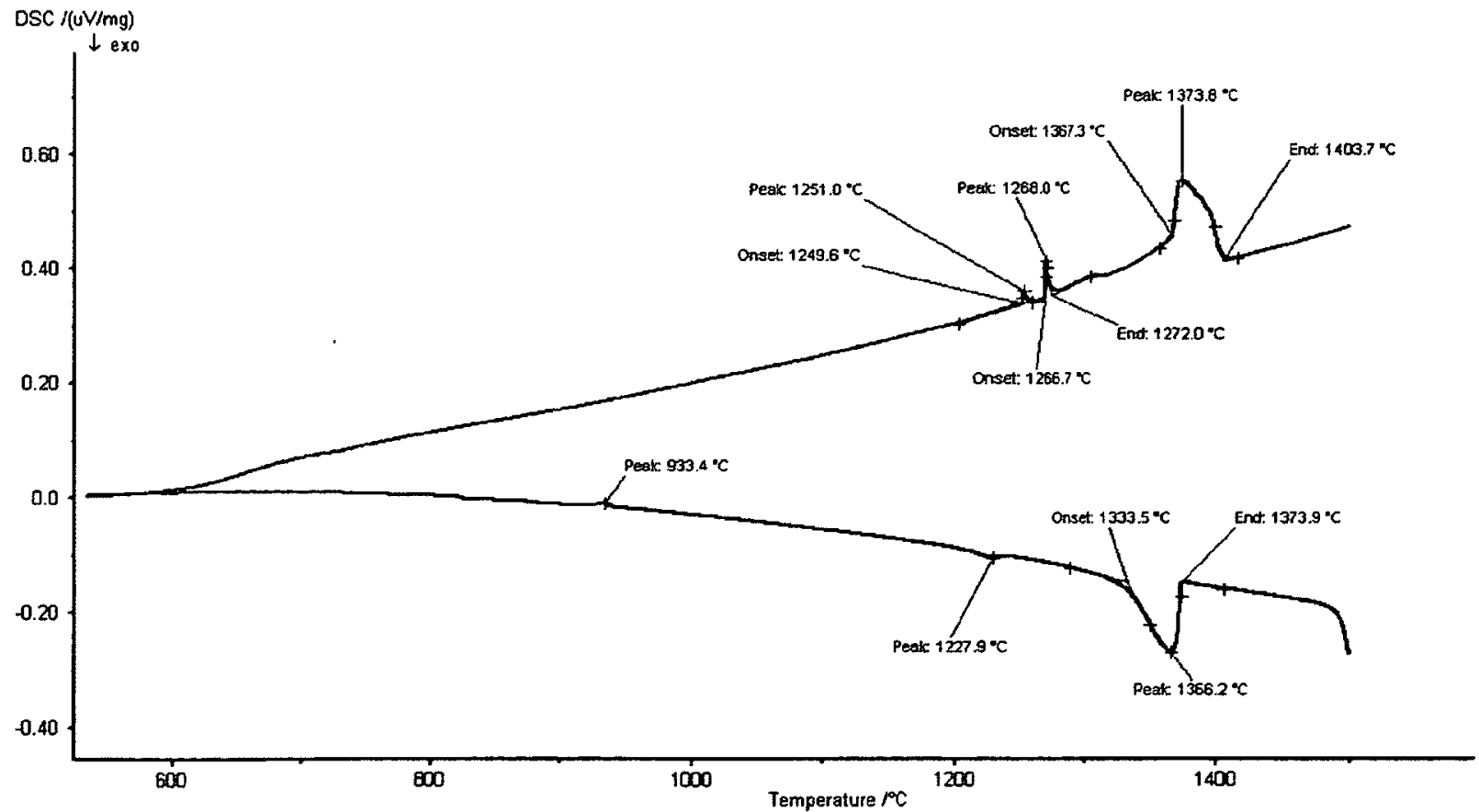

(c) 


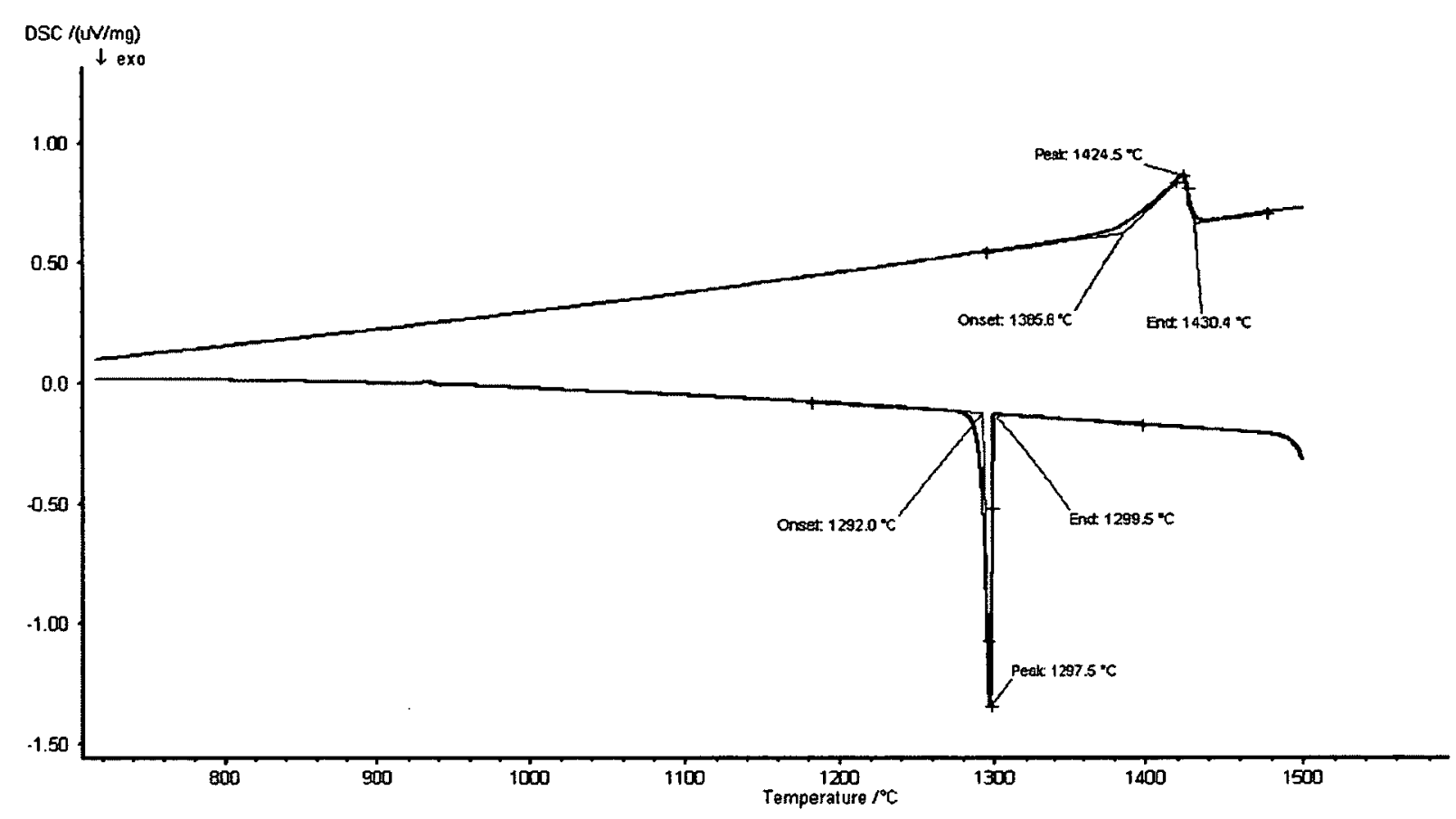

(d)

Fig. 3.6. DSC thermograms: (a) specimen A, (b) specimen B, (c) specimen C and (d) specimen $\mathrm{K}$.

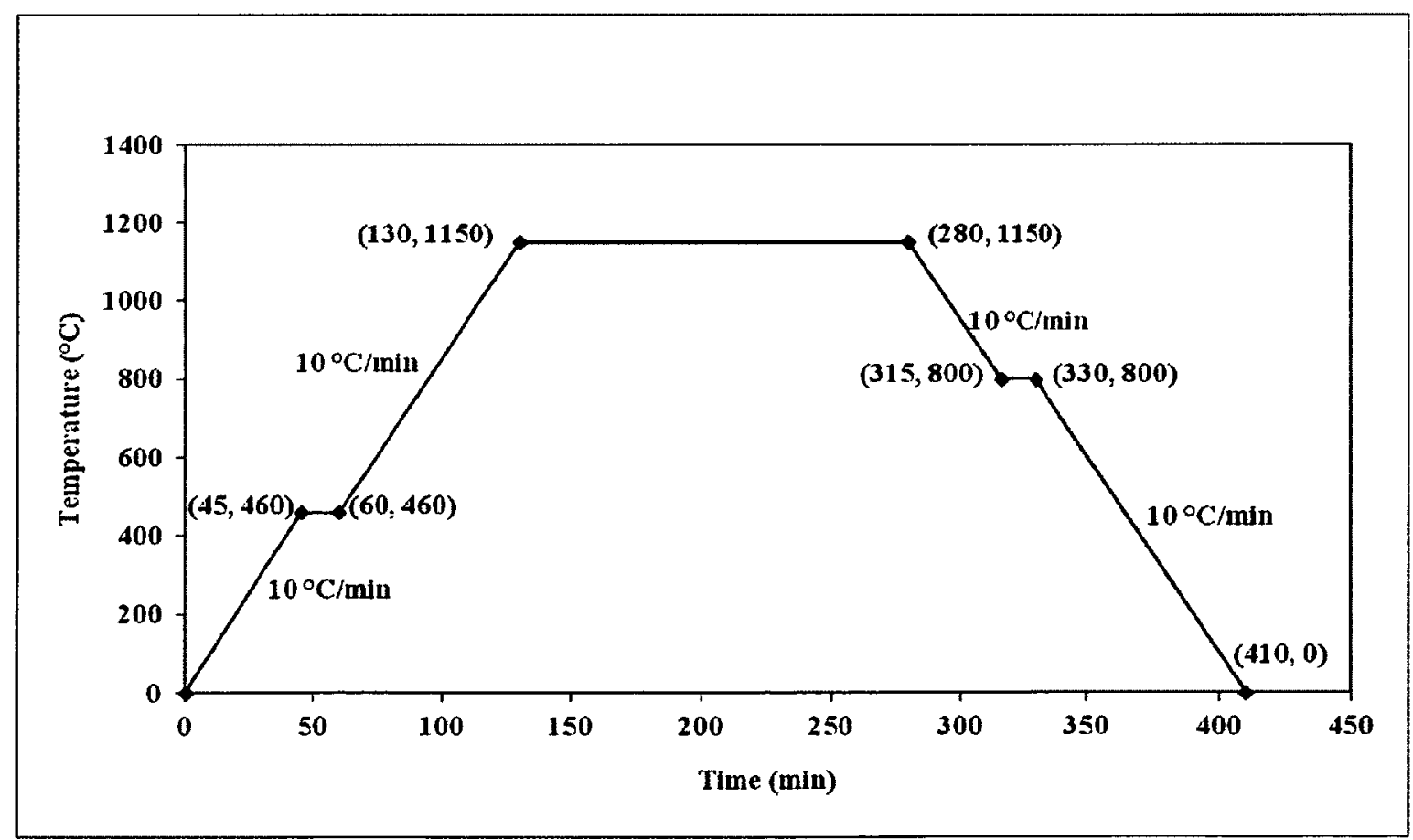

Fig. 3.7. Sintering cycle for pure Stellite 25 and Stellite 25 based composites. 


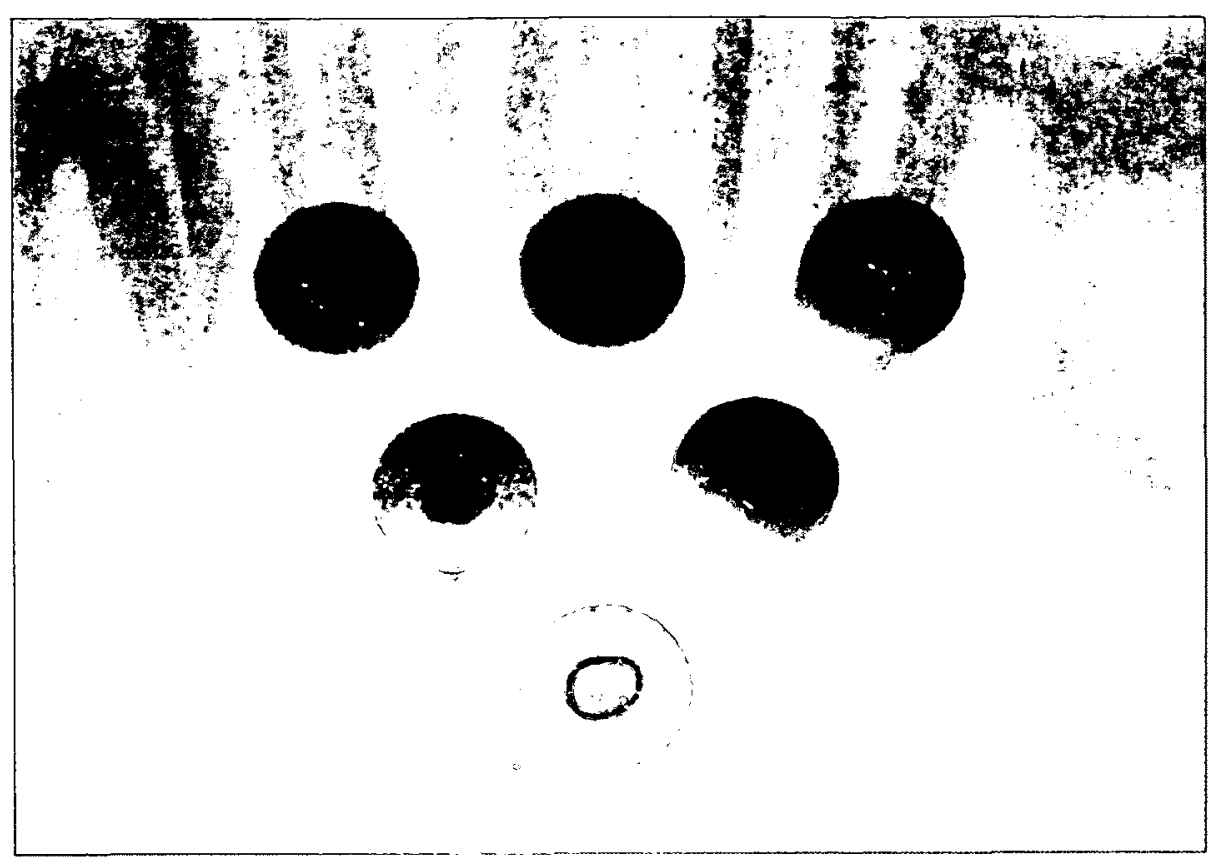

Fig. 3.8. Mounted sintered specimens.

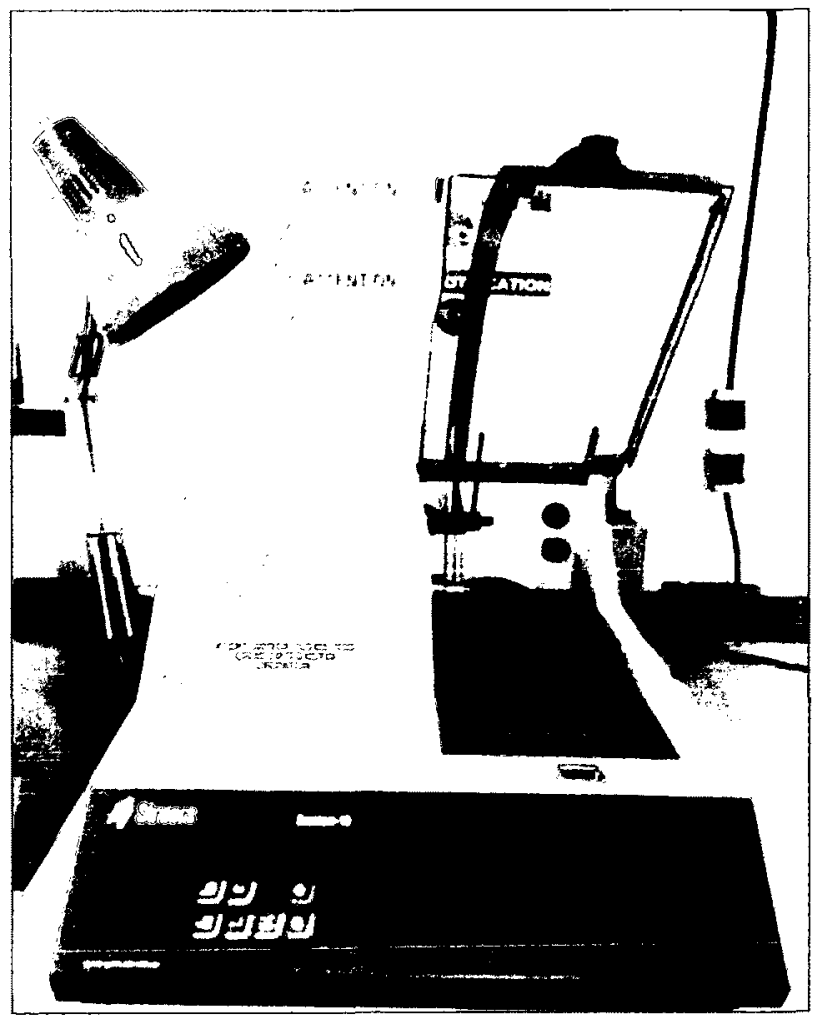

Fig. 4.1. Struers Secotom-10 cutting machine. 


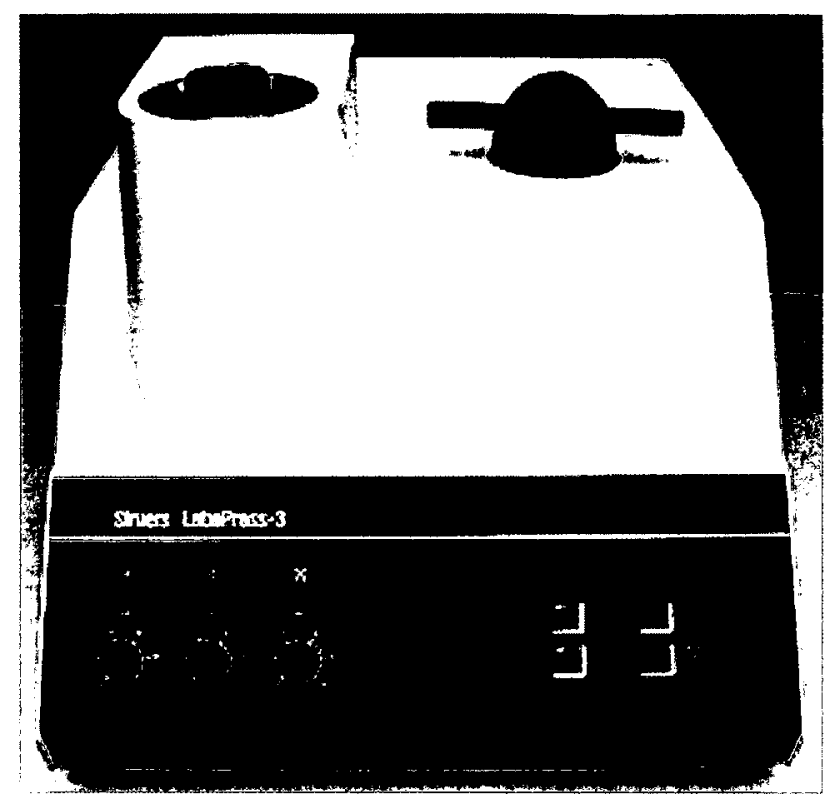

Fig. 4.2. LaboPress-3 mounting press (Courtesy of Struers Co.).

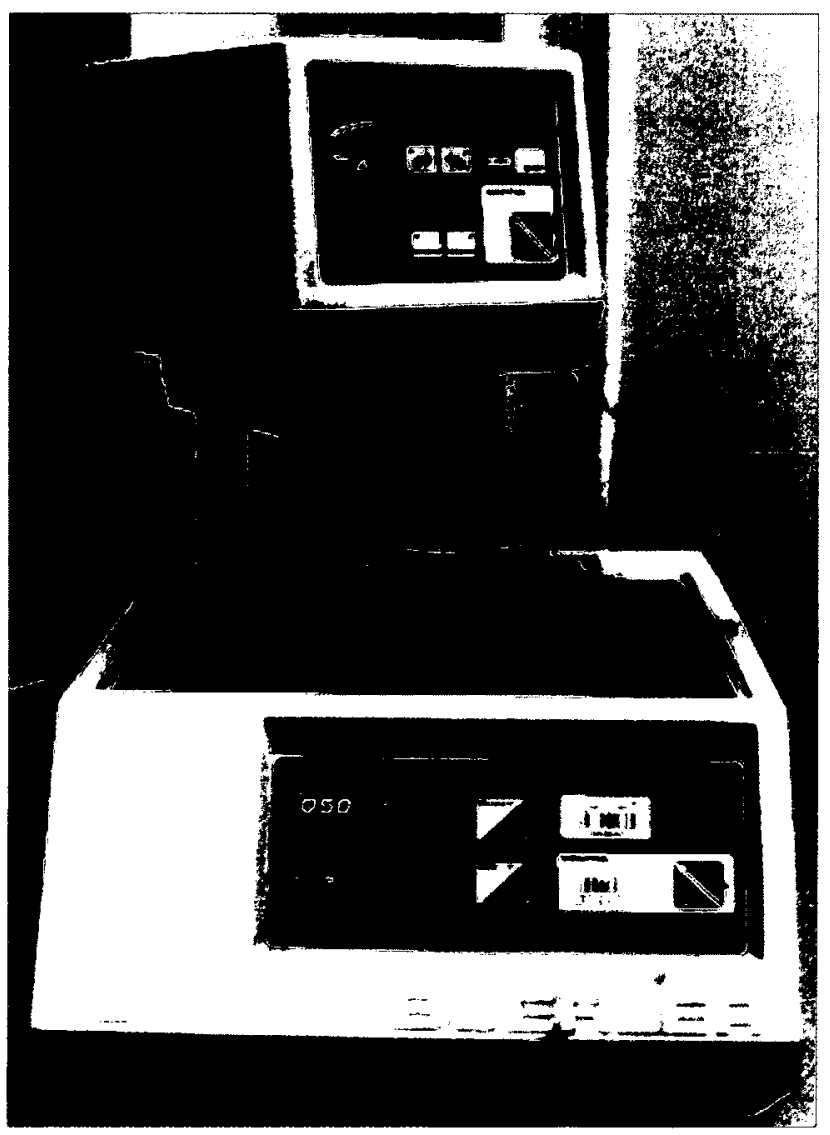

Fig. 4.3. Buehler Ecomet-4 semiautomatic grinder polisher. 


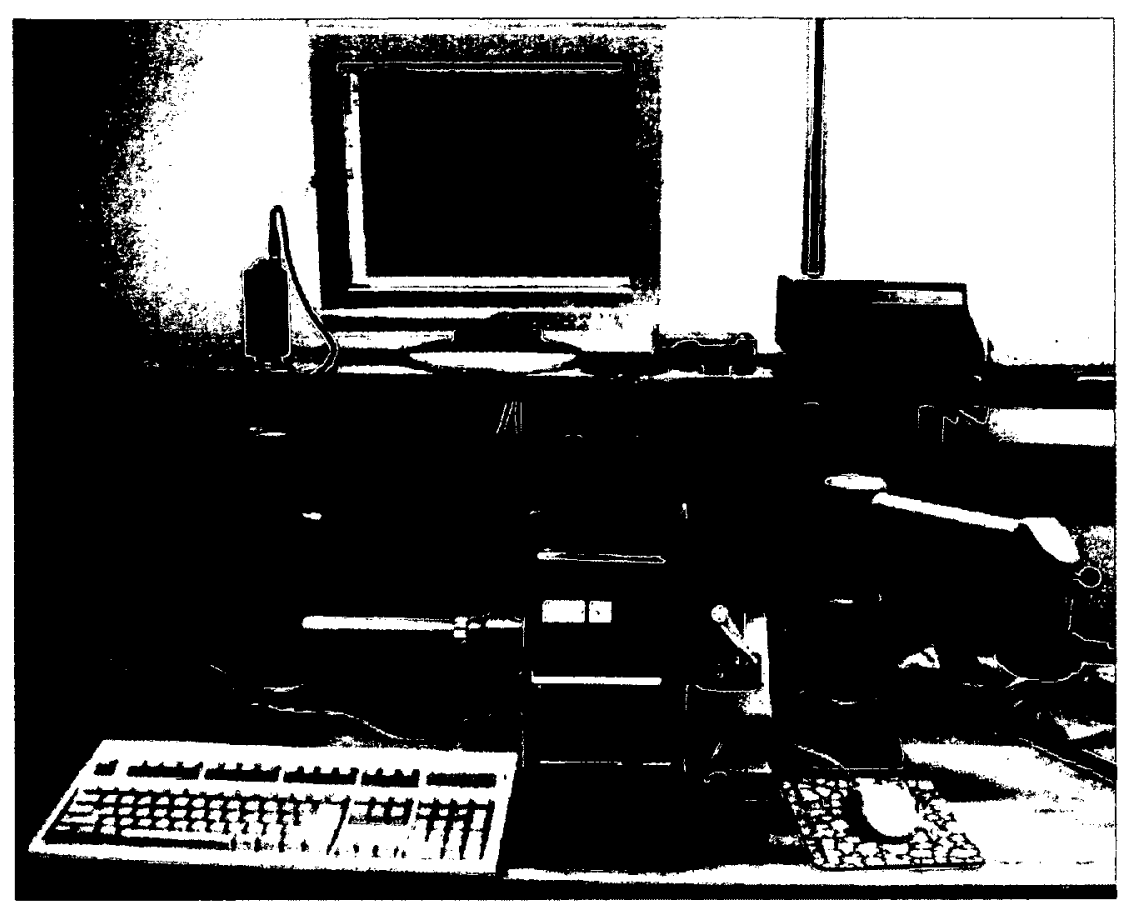

Fig. 4.4. Olympus PM-63 optical microscope.

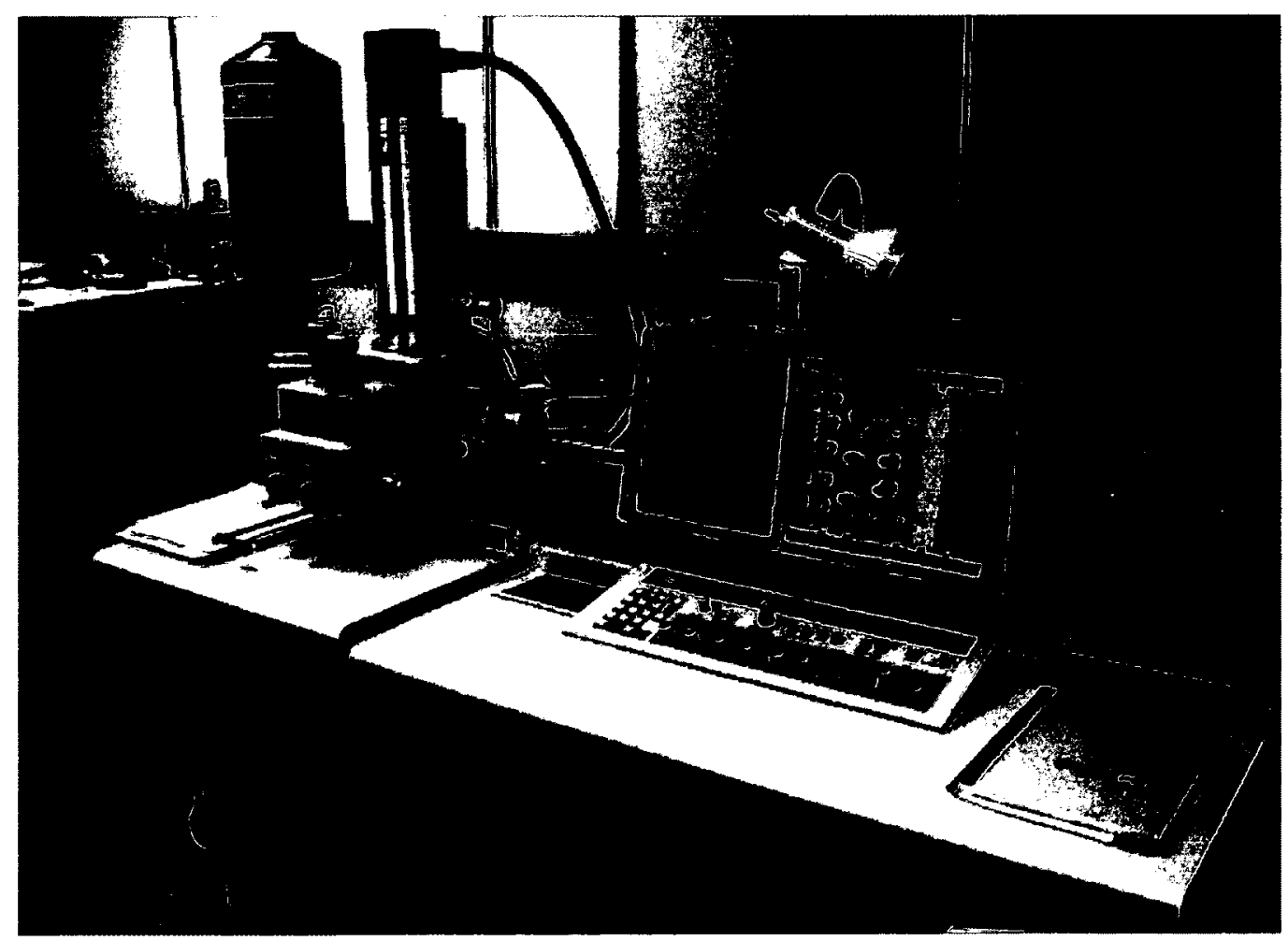

Fig. 4.5. Hitachi S-570 scanning electron microscope. 


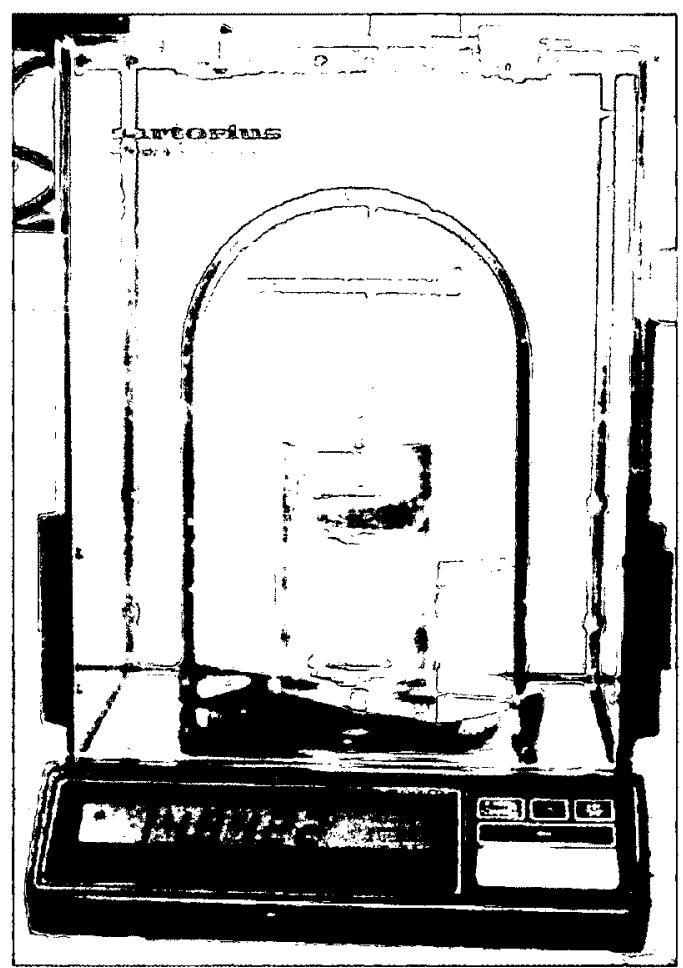

Fig. 4.6. Density measurement setup.

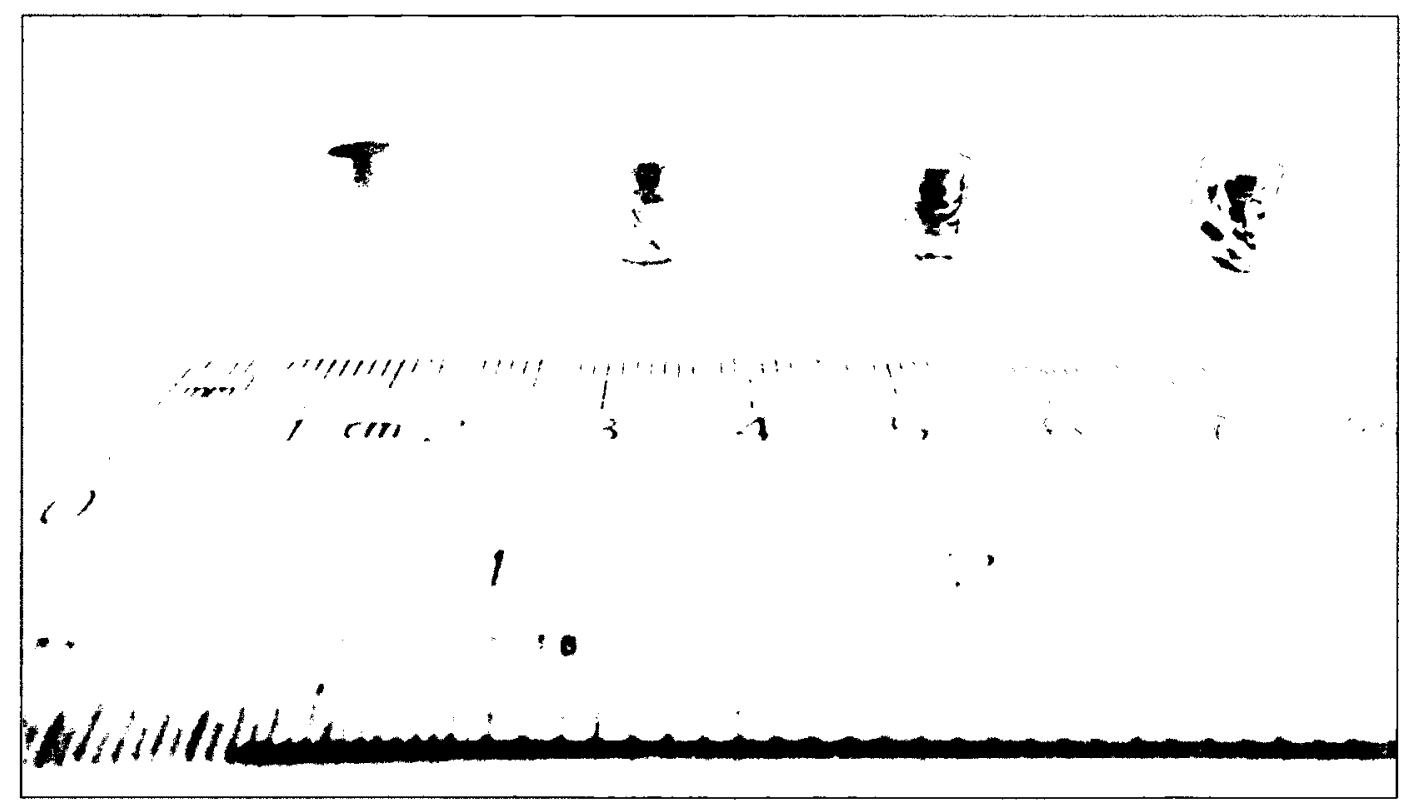

Fig. 4.7. Immersion corrosion test specimens. 


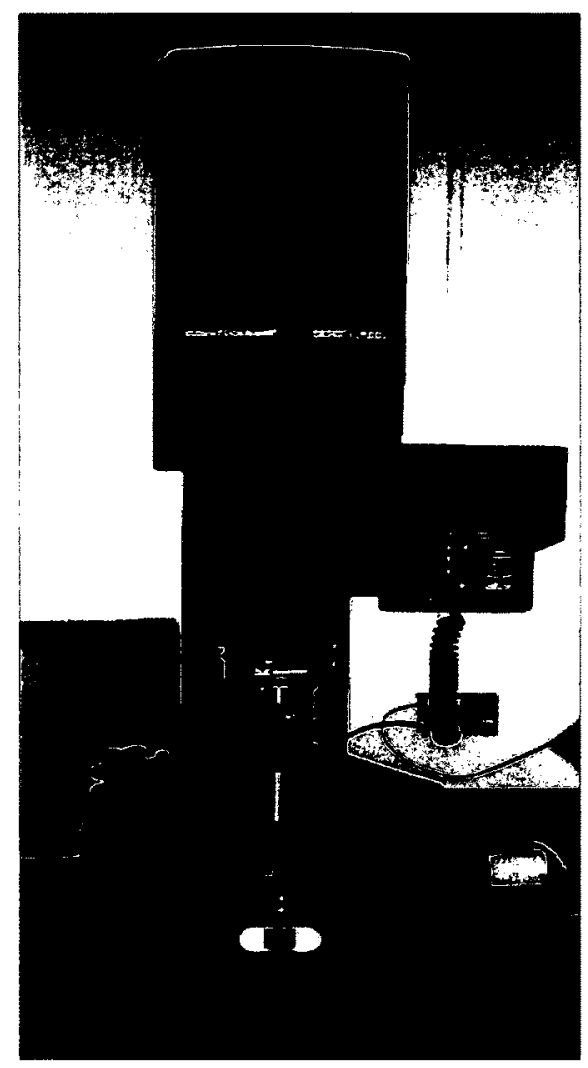

Fig. 4.8. Rockwell hardness test apparatus.

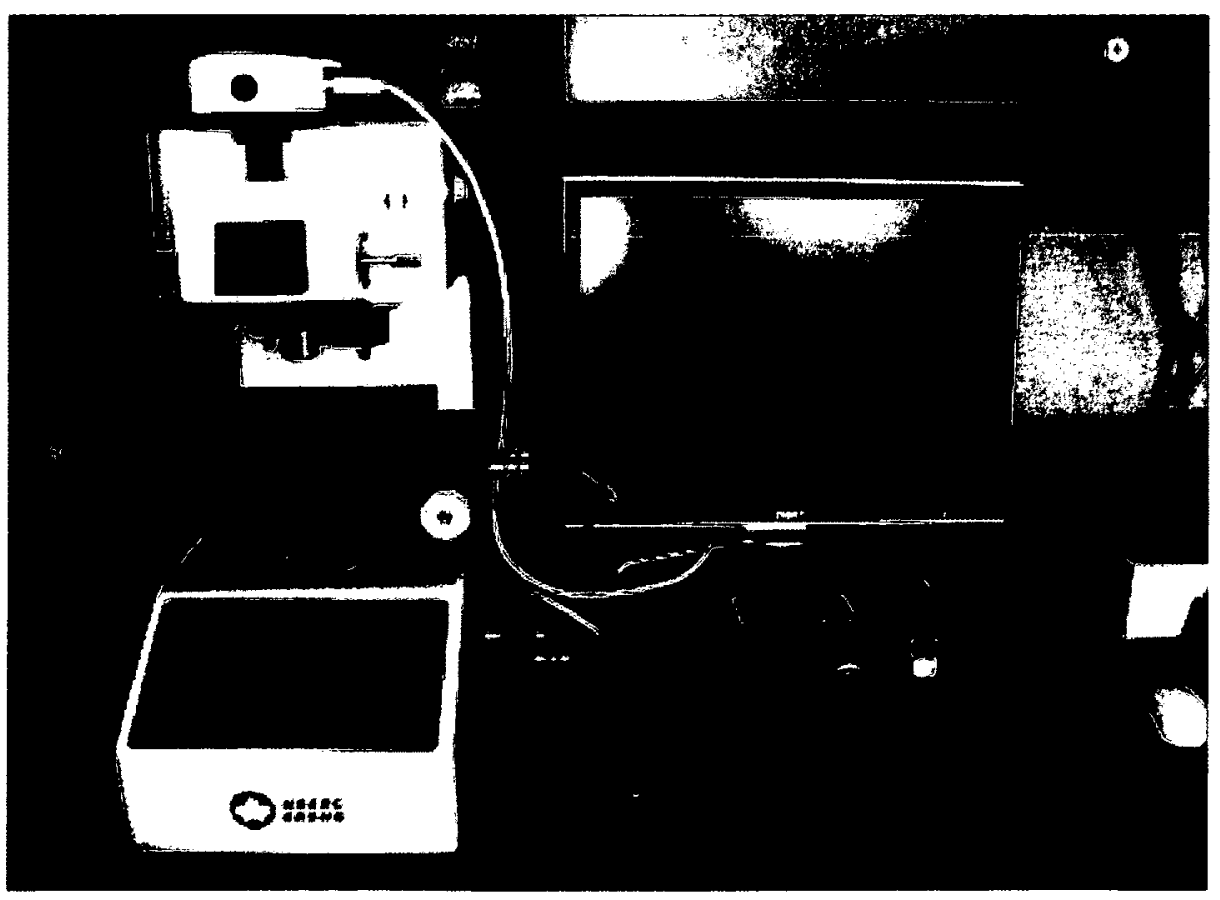

Fig. 4.9. SMT-X7 dual indenter microhardness tester. 


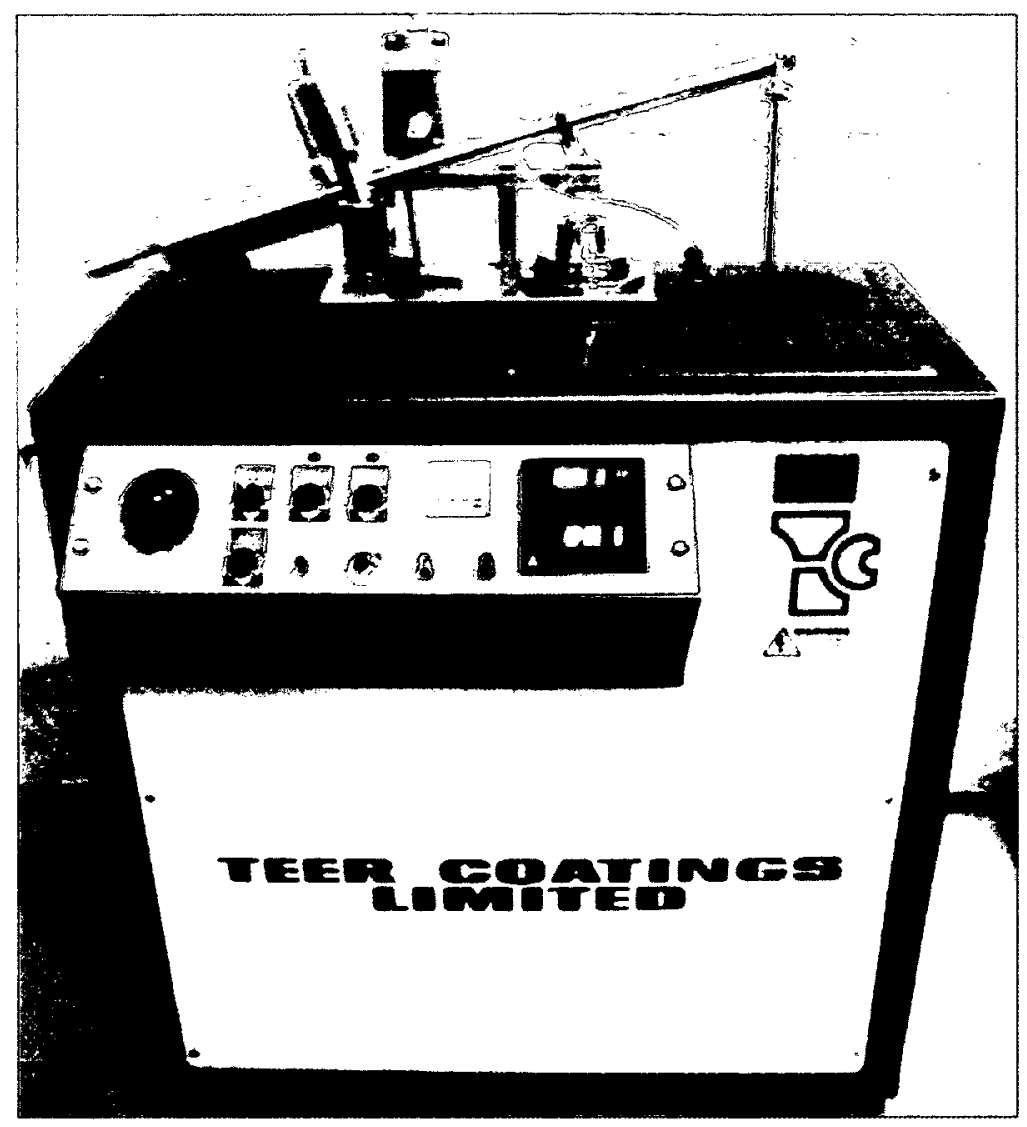

Fig. 4.10. TEER-POD-2 computer controlled pin-on-disk tribometer.

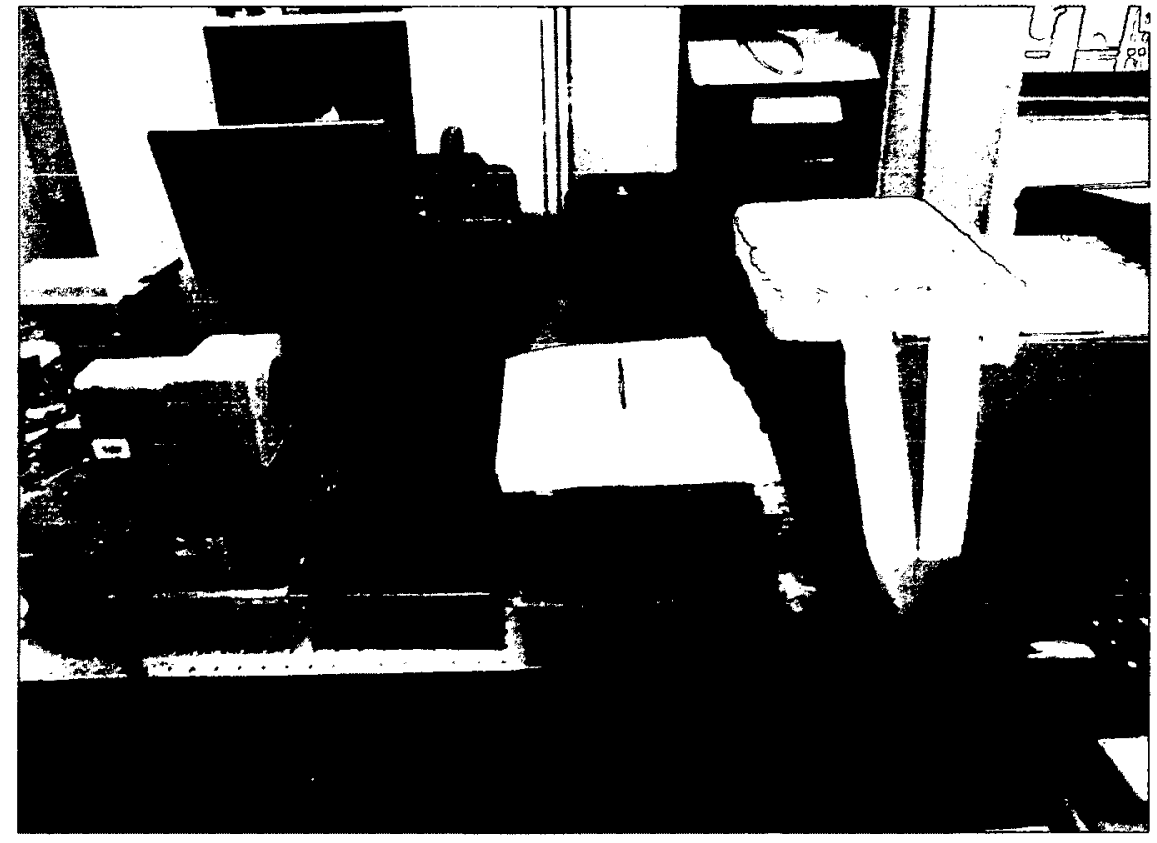

Fig. 4.11. Pulsed thermography test setup. 


$$
\cong
$$




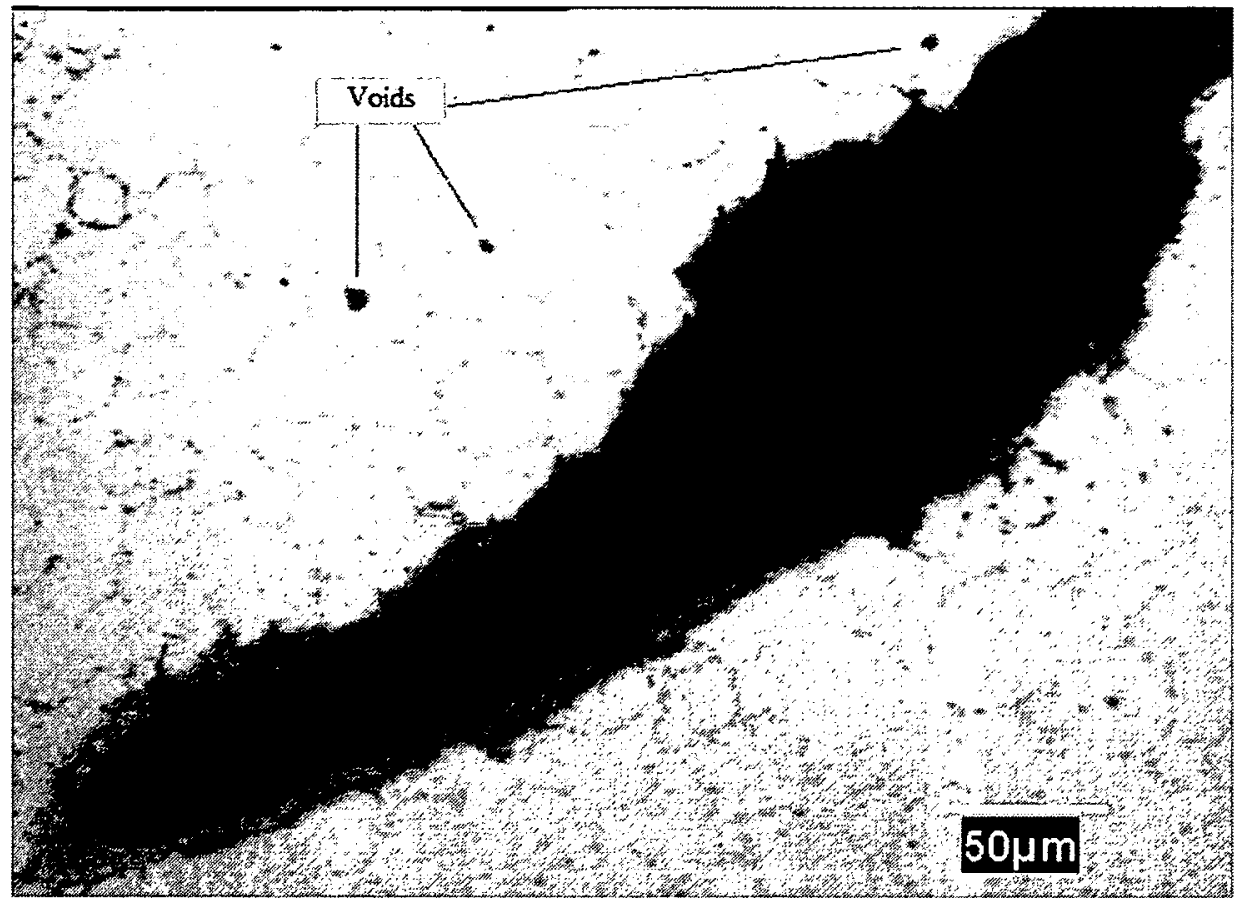

(c)

Fig. 5.1. Optical microstructural images of the specimens in an unetched condition: (a) pure Stellite 25 (specimen A), (b) composite specimen F and (c) presence of voids in the composite specimen (specimen C). 


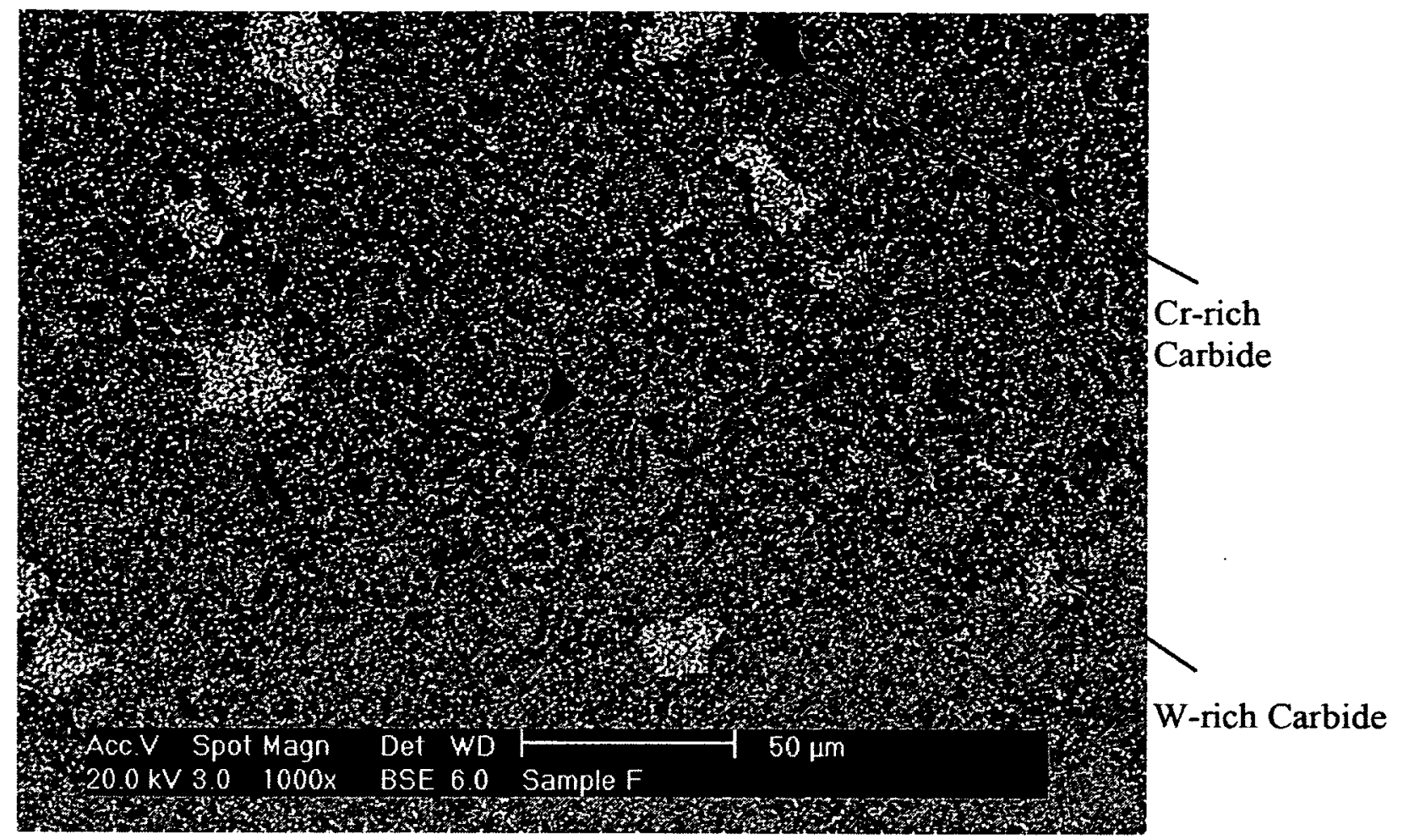

Fig. 5.2. SEM image of the etched composite $F$ in an area close to carbon fibres.

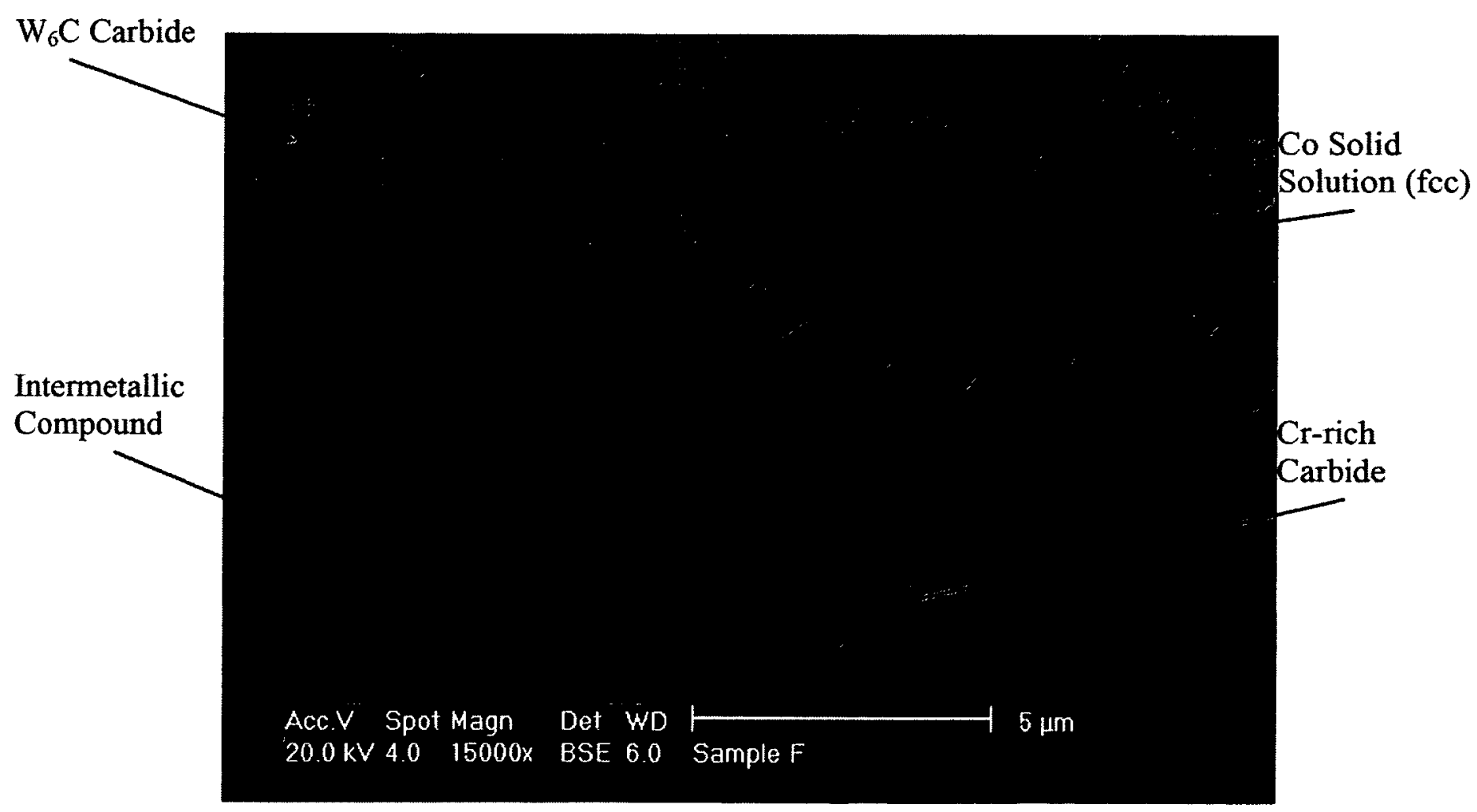

(a) 
Sample F, white phase

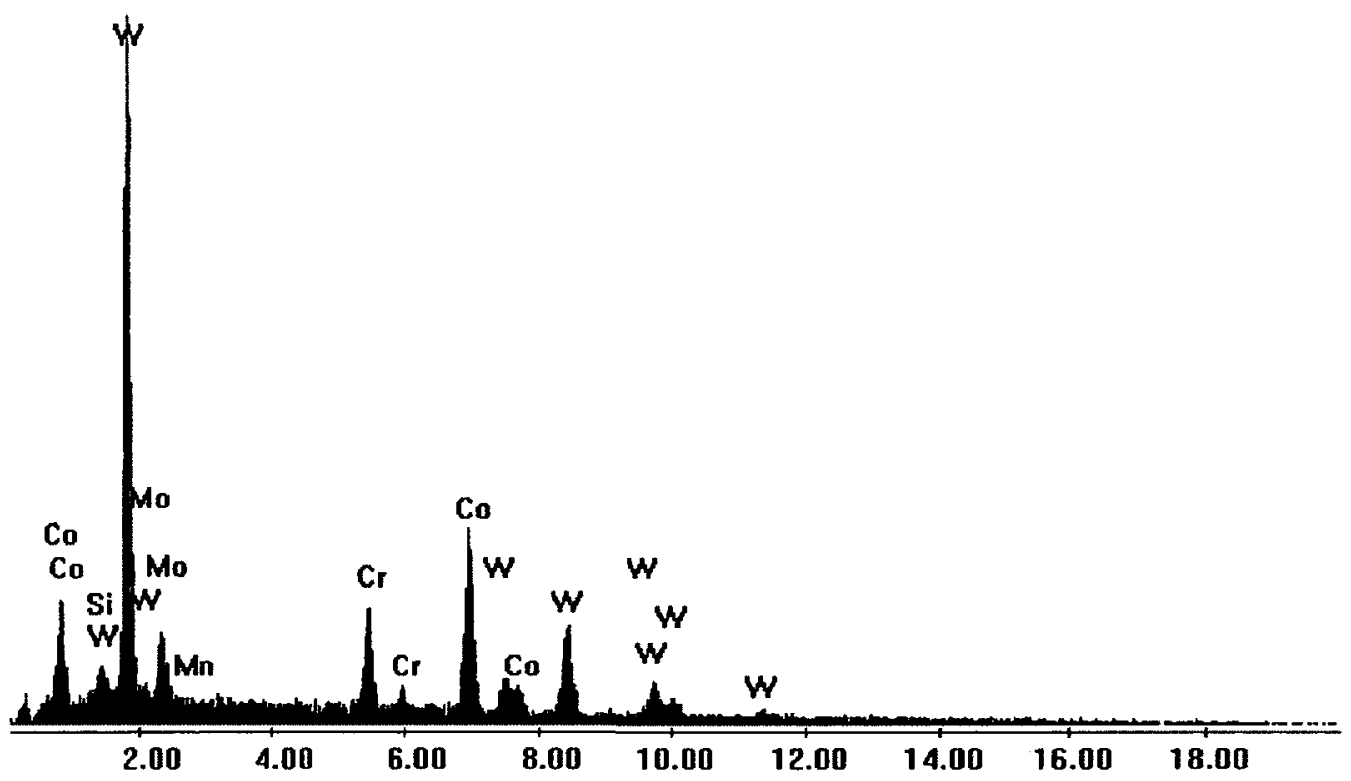

(b)

Sample F, gray phase

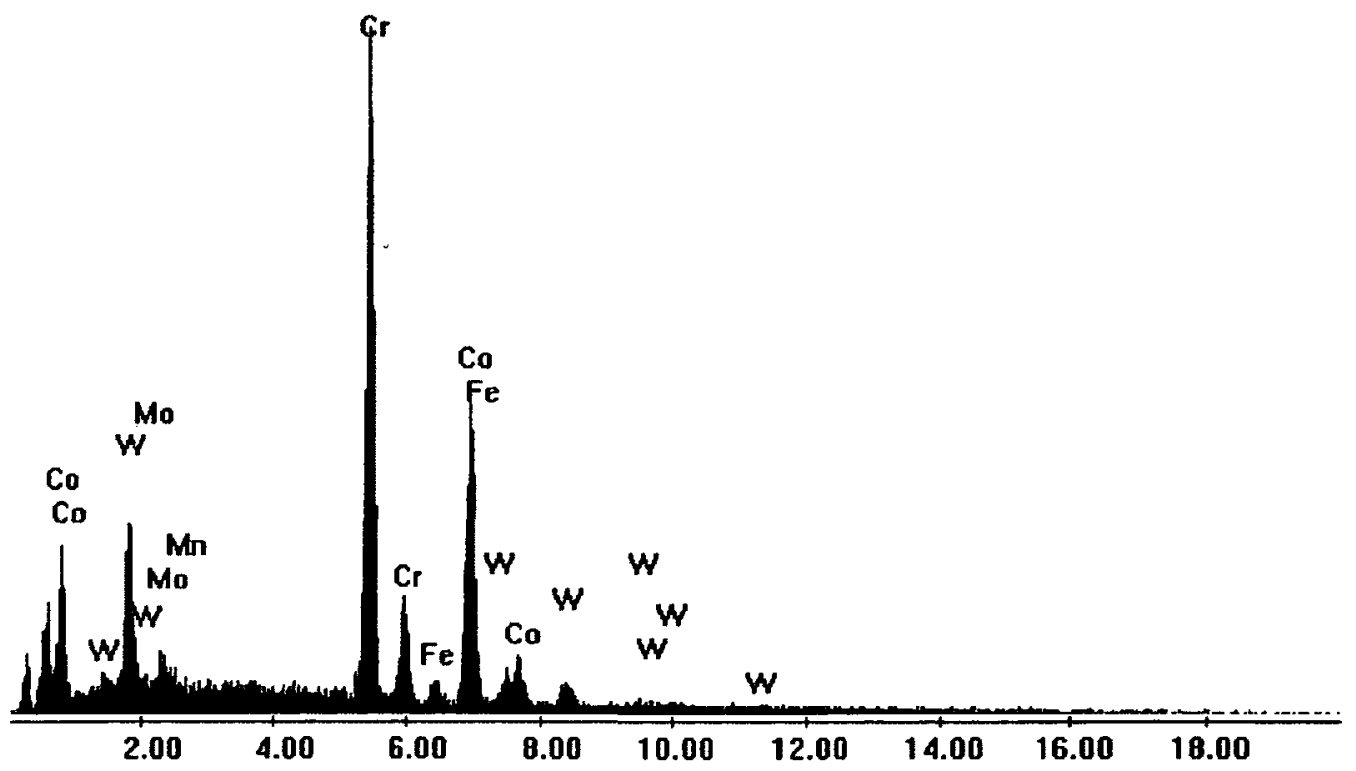

(c) 


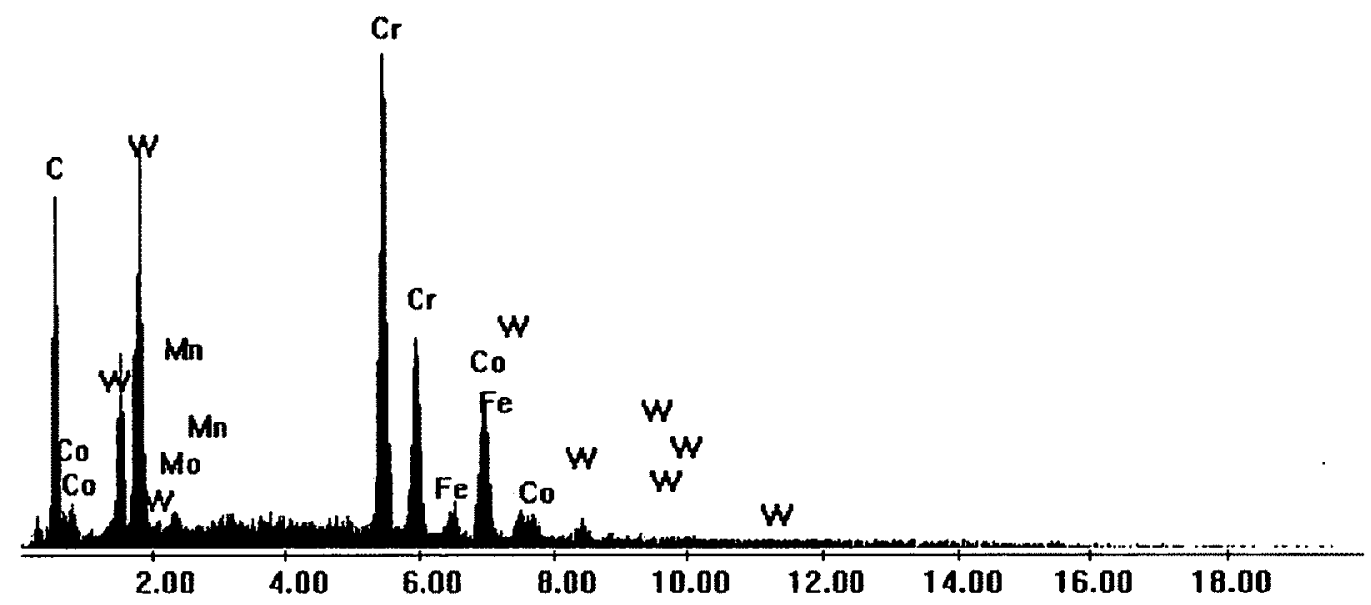

(d)

Fig. 5.3. SEM/EDX analysis of specimen F: (a) SEM microstructural image at high magnification, (b) EDX spectrum of white phase, (c) EDX spectrum of dark grey phase and (d) EDX spectrum of black phase. 


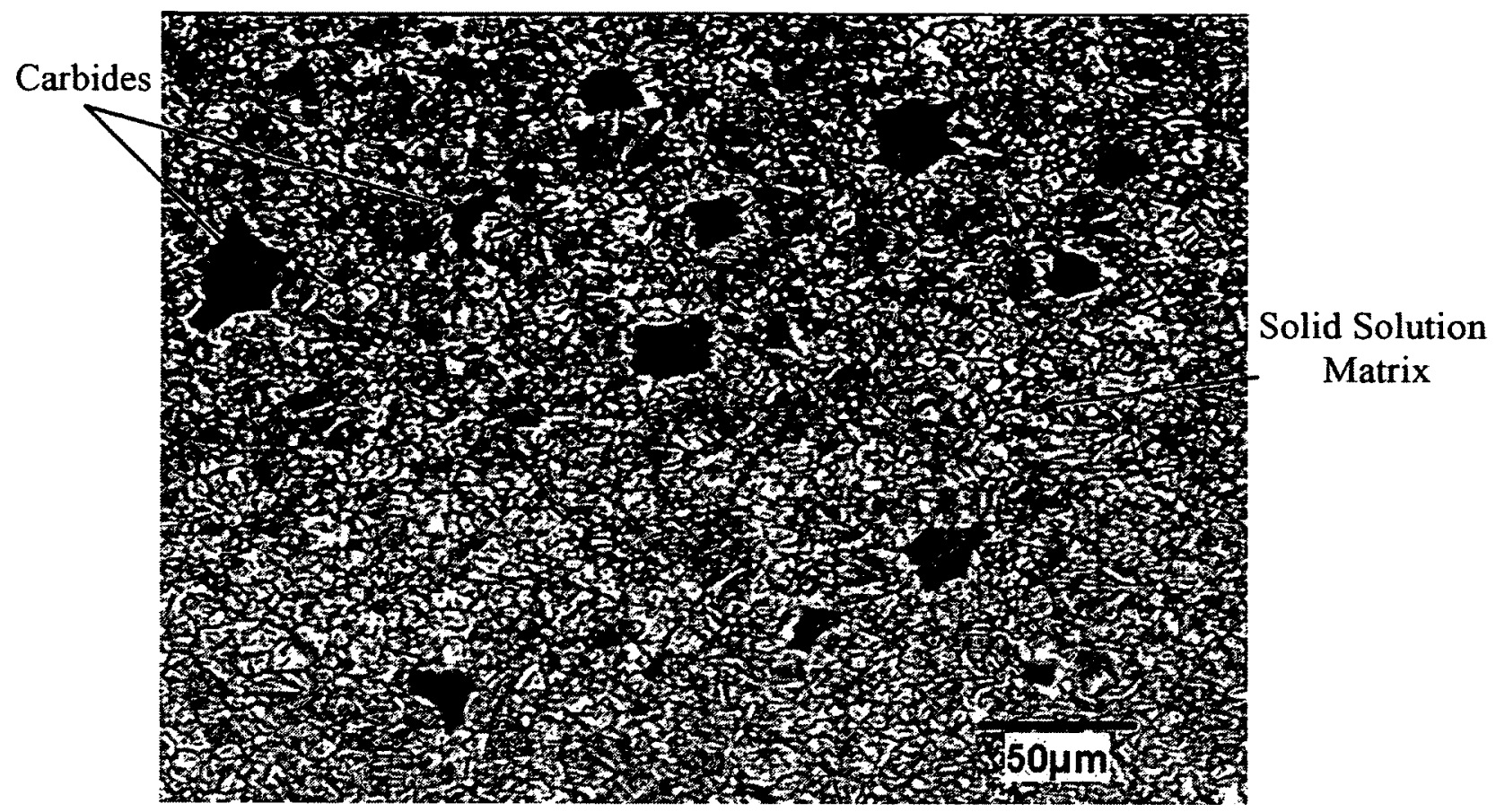

Fig. 5.4. Optical microstructural image of the etched pure Stellite 25 specimen. 
(q)
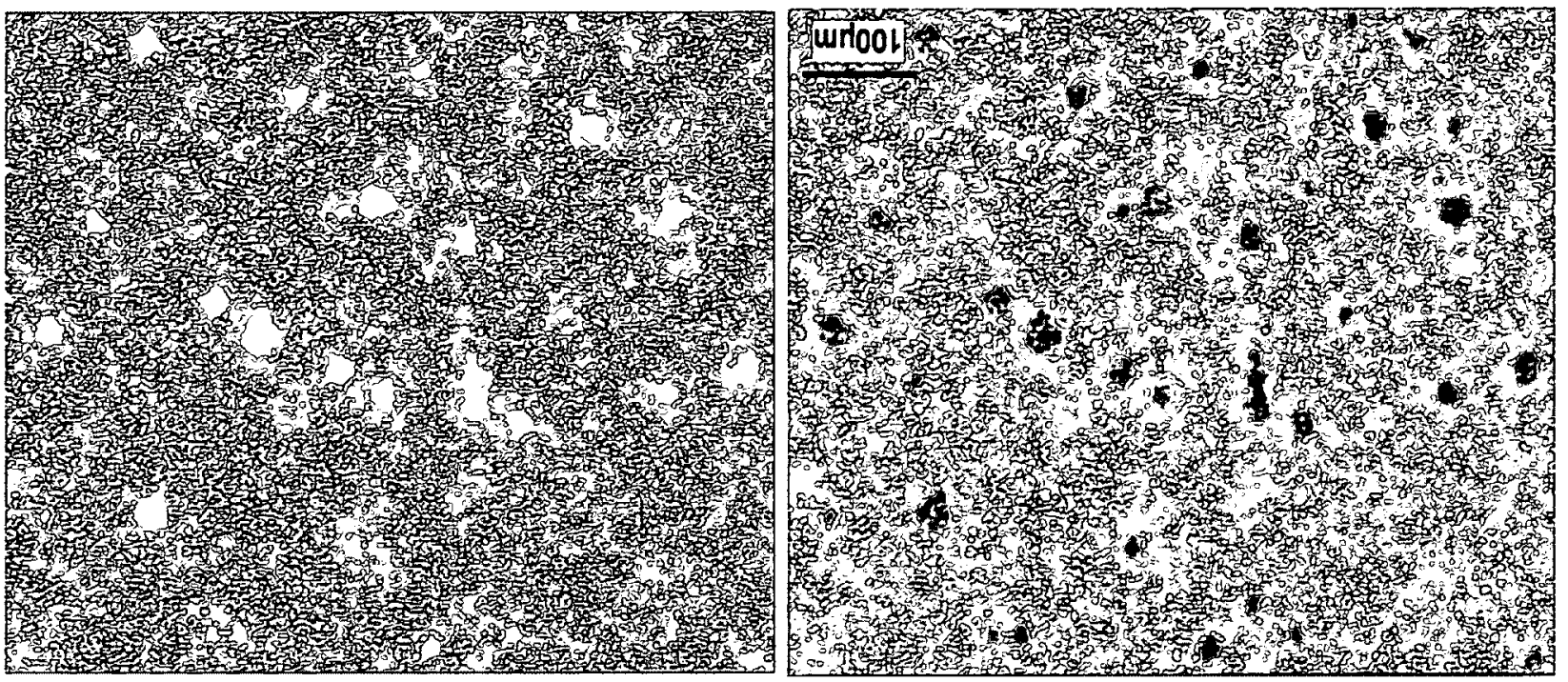

(e)
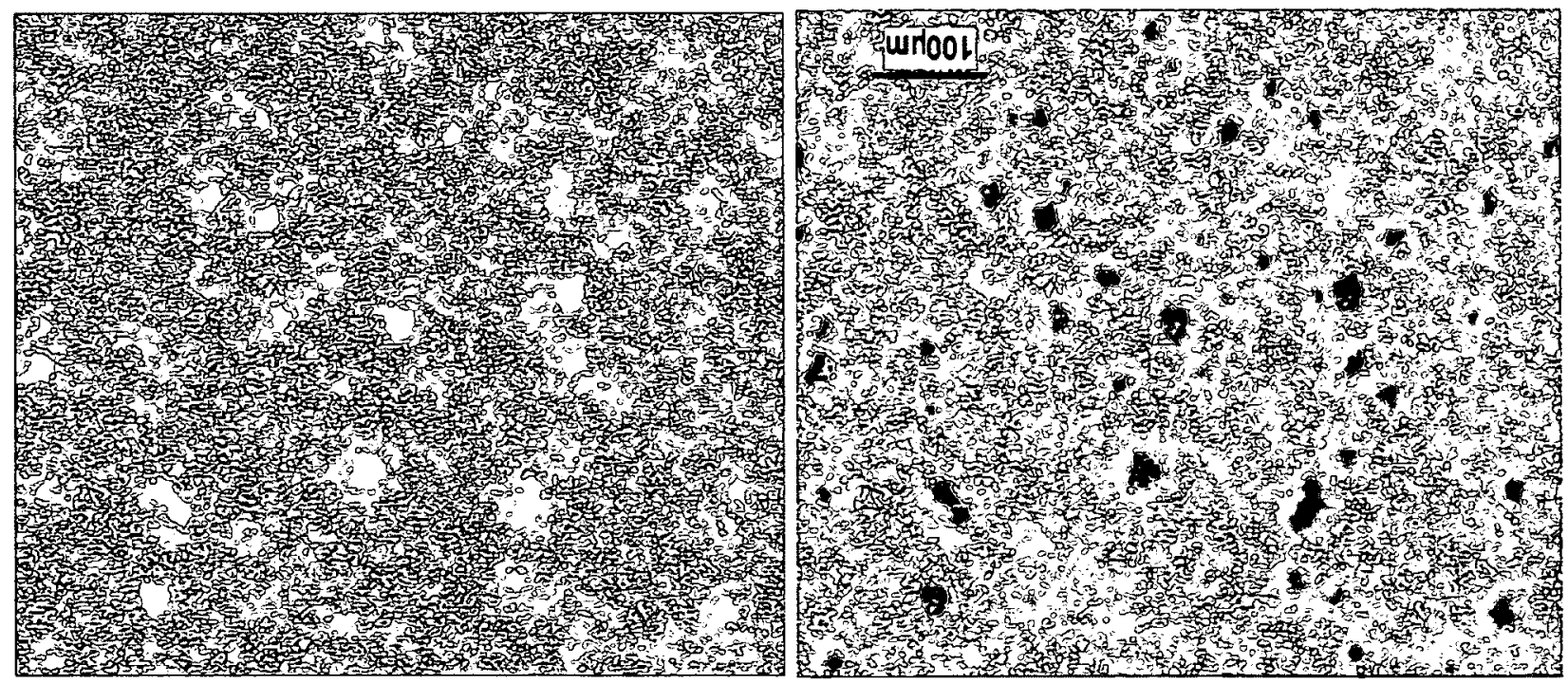


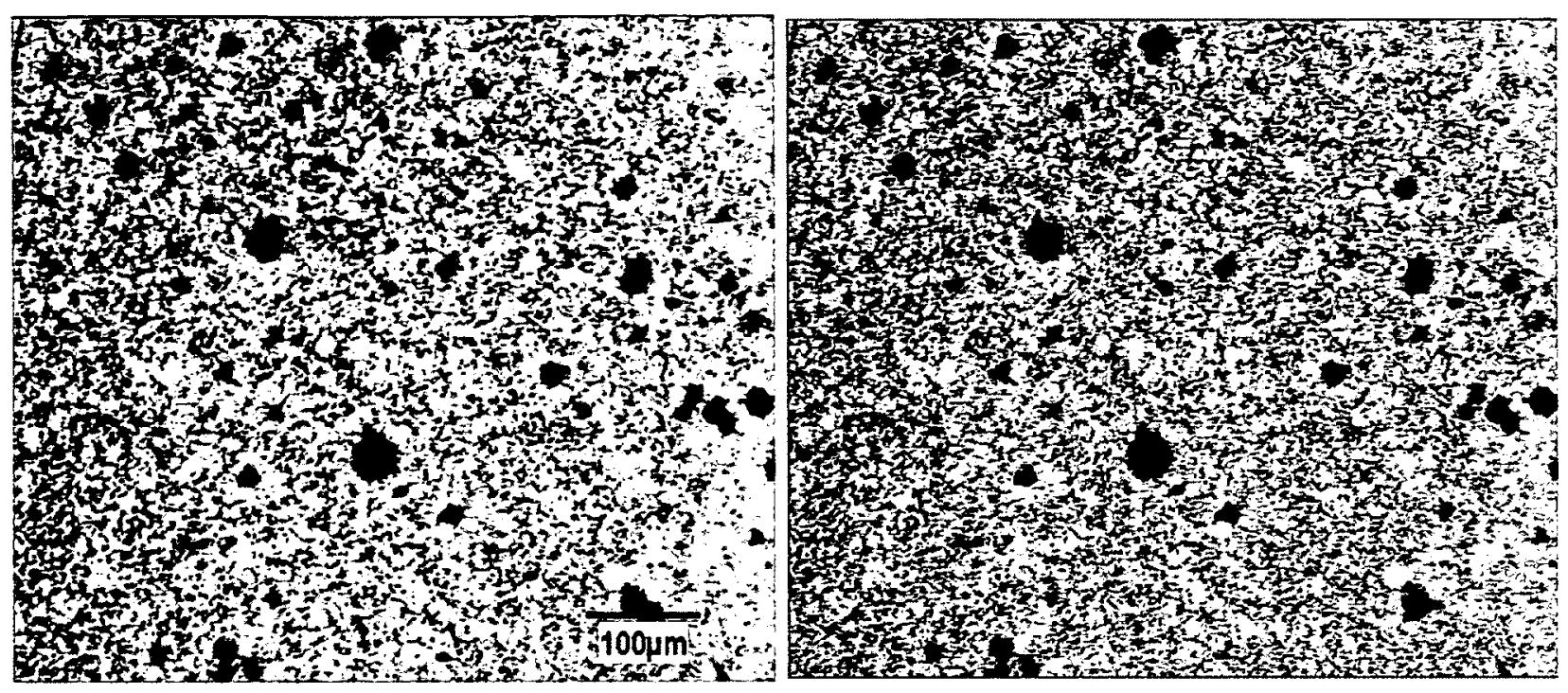

(c)

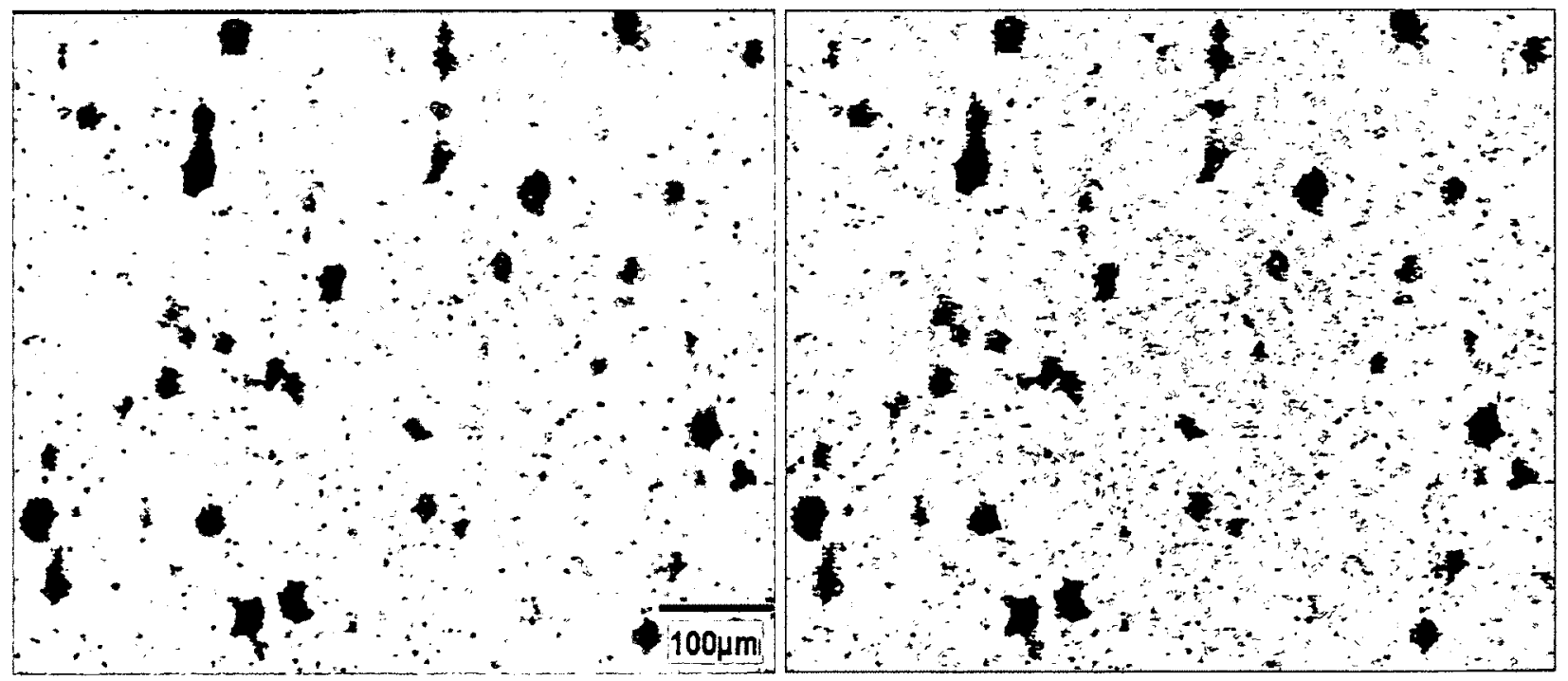

(d) 

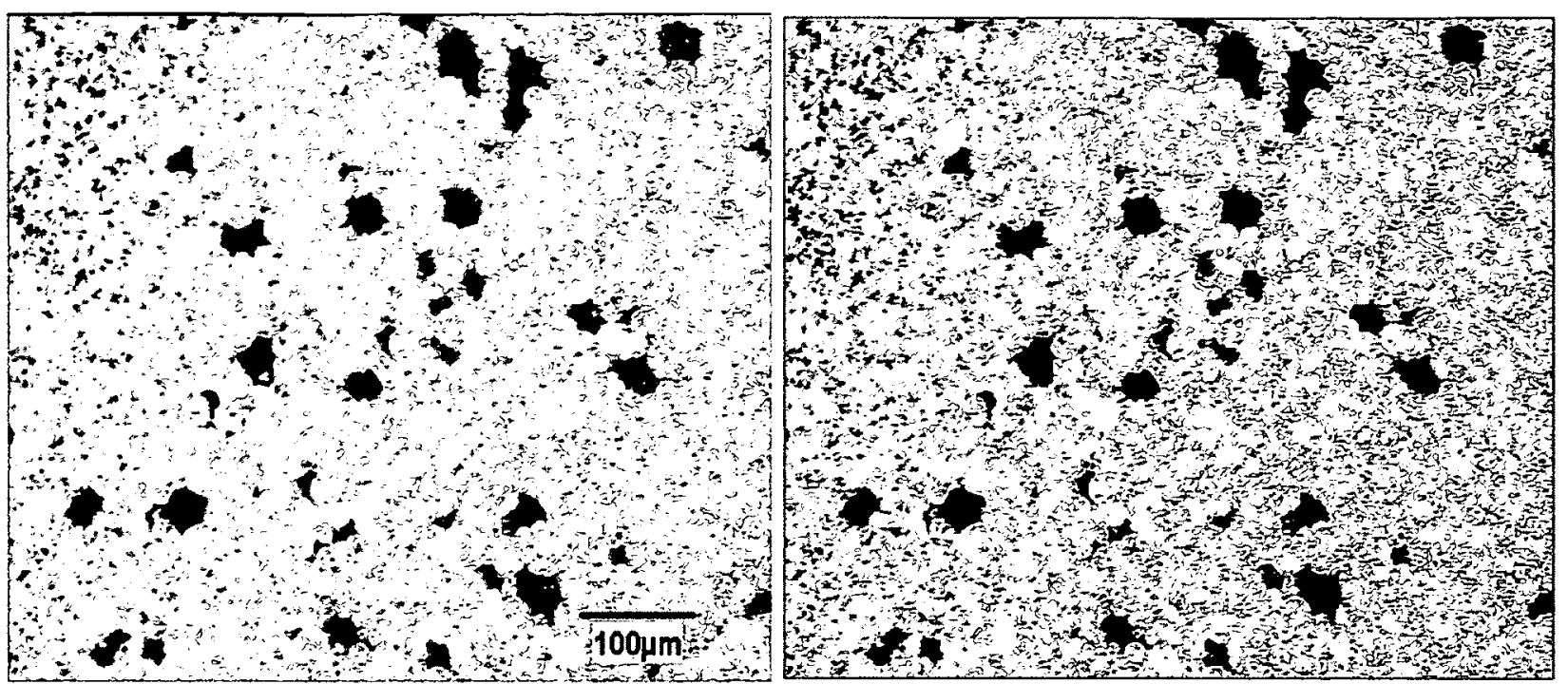

(e)
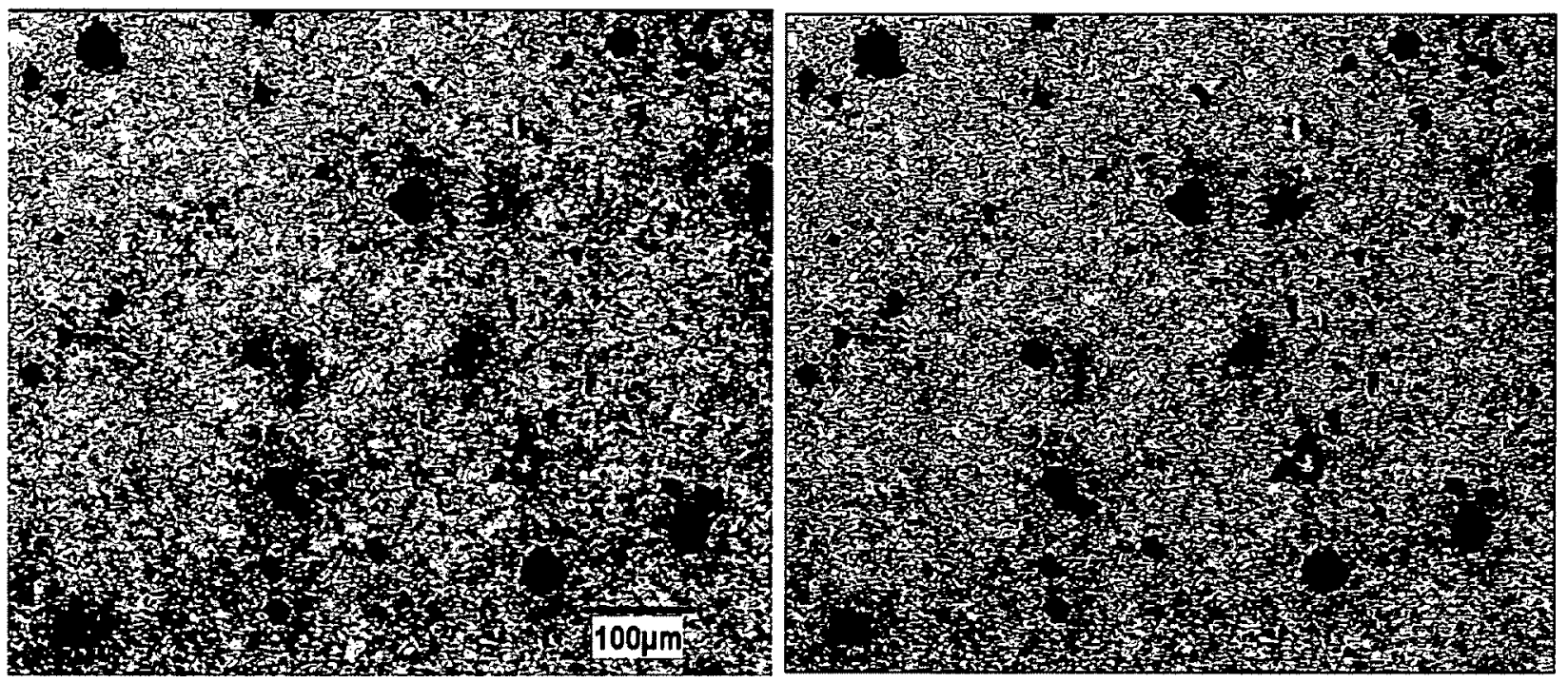

(f)

Fig. 5.5. Microstructural pictures used for the evaluation of carbide volume fraction: (a) specimen $A$, (b) specimen $B$, (c) specimen $C$, (d) specimen $D$, (e) specimen $E$ and (f) specimen $F$. 


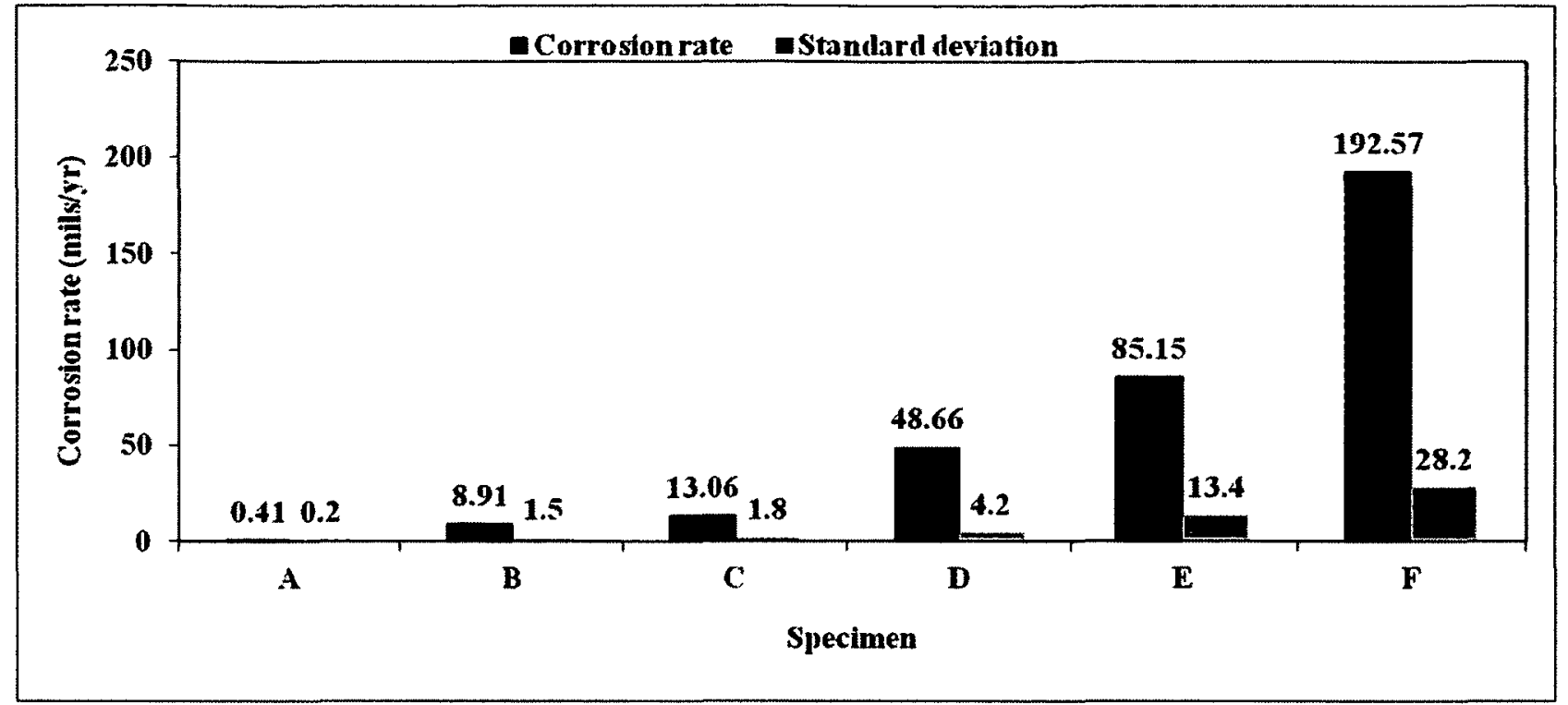

Fig. 5.6. Corrosion resistances of plain carbon fibre reinforced specimens in oxidizing acid $65 \% \mathrm{HNO}_{3}$ at boiling temperature.

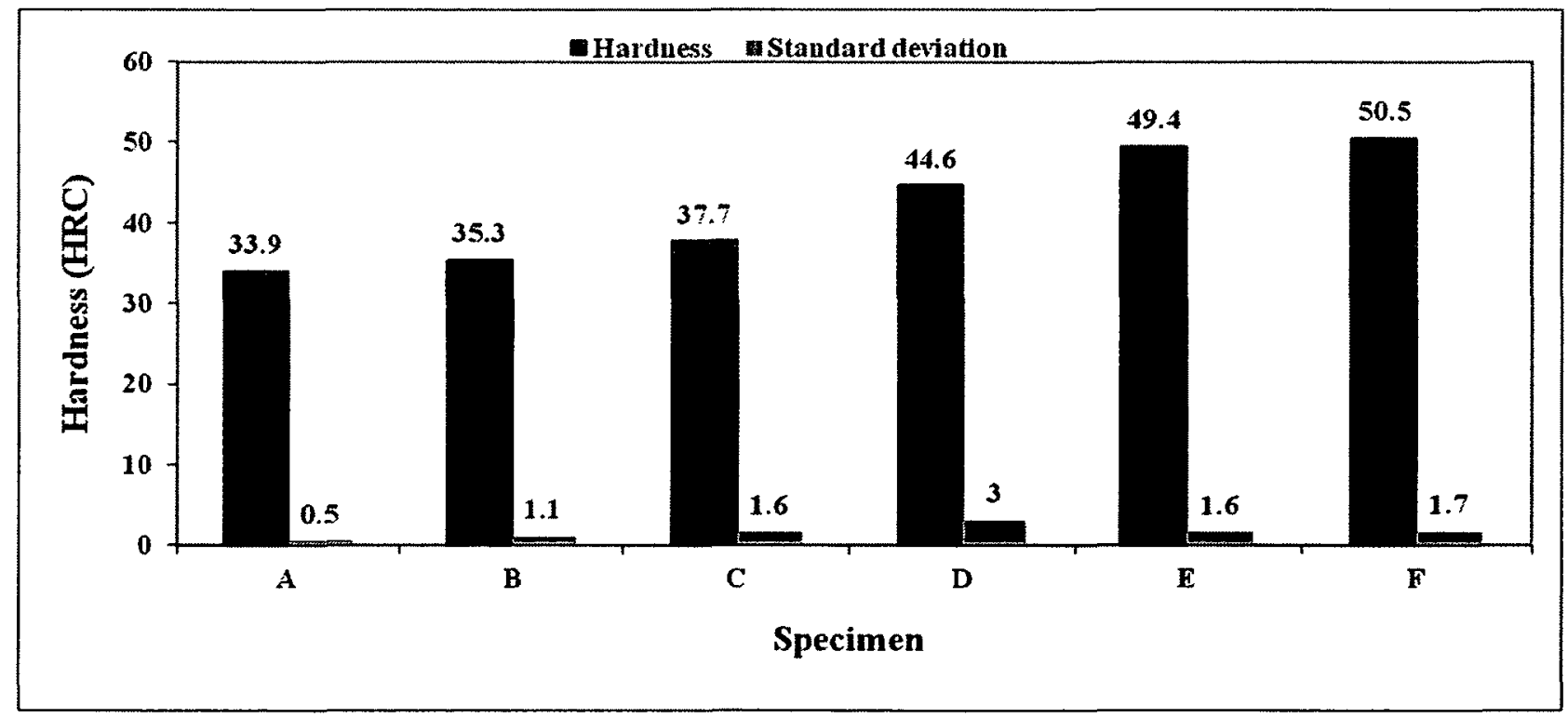

Fig. 5.7. Rockwell hardnesses of plain carbon fibre reinforced specimens. 


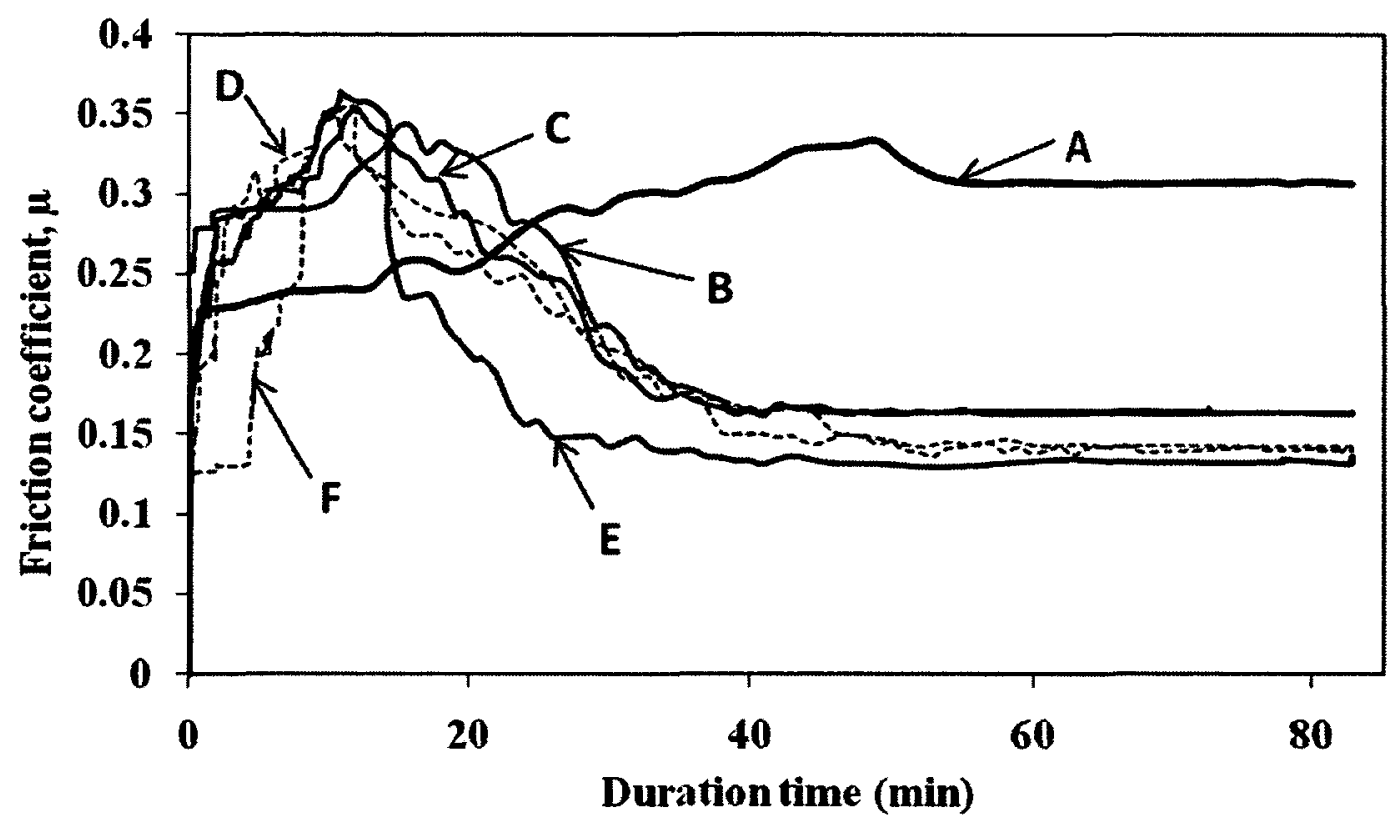

Fig. 5.8. Variations of friction coefficient of plain carbon fibre reinforced specimens with sliding time.

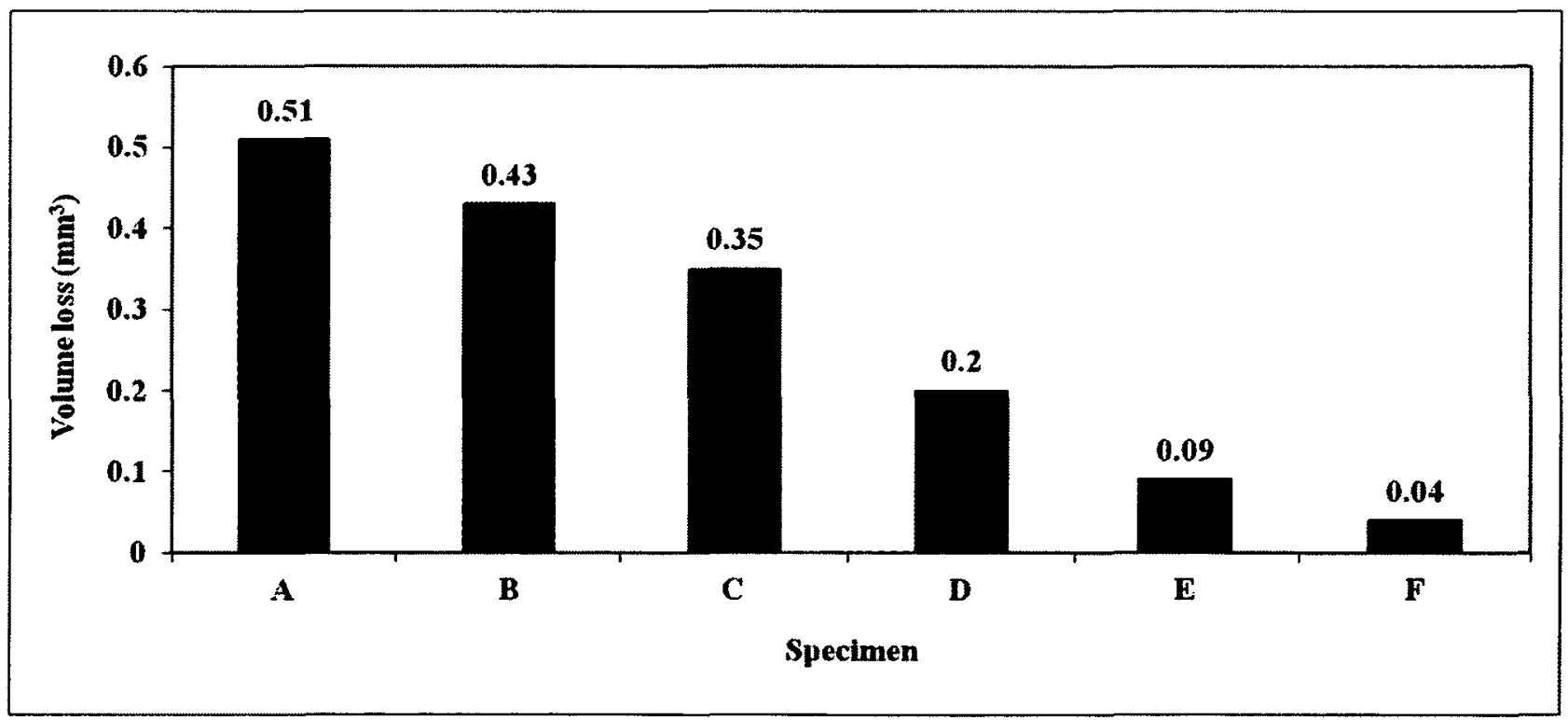

Fig. 5.9. Volume losses of plain $\mathrm{CF}$ reinforced specimens due to sliding wear. 


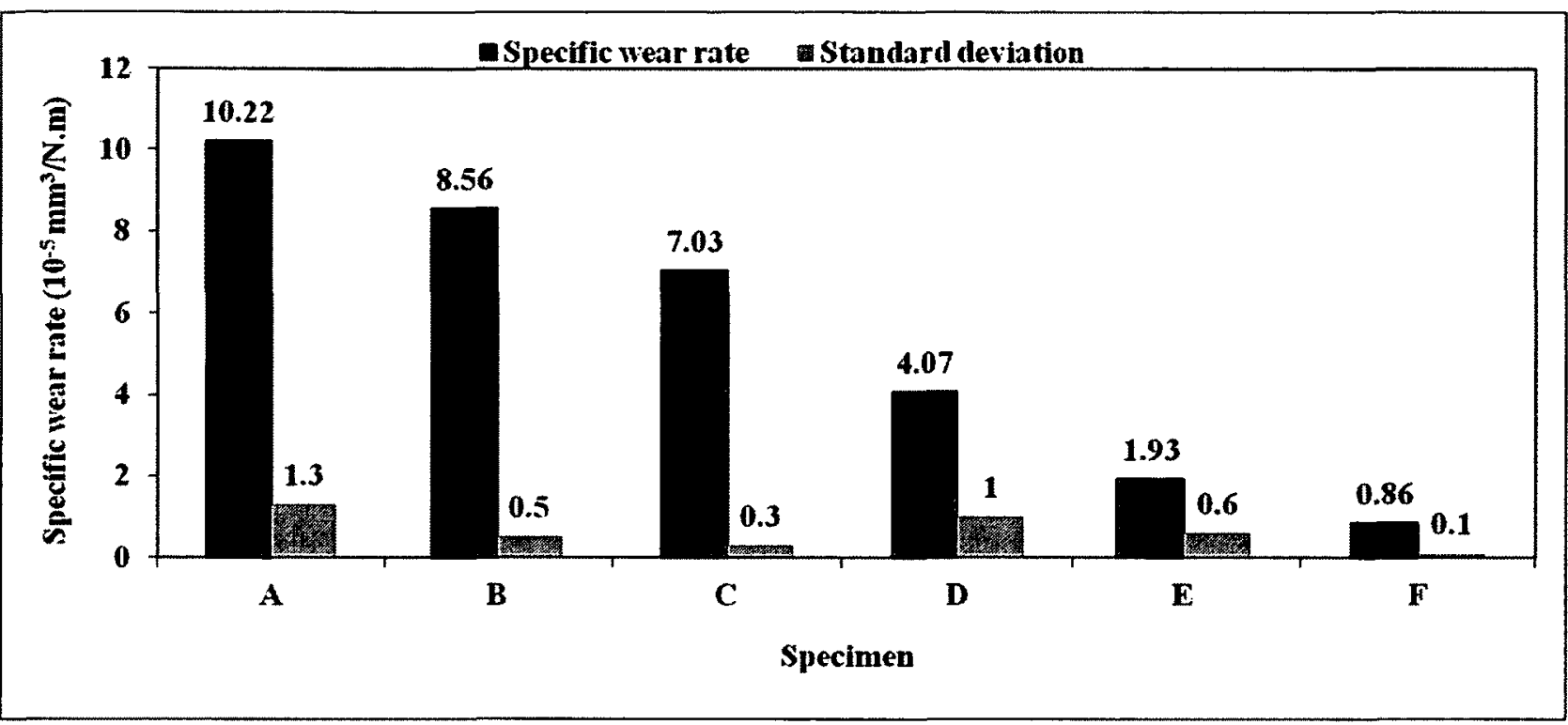

Fig. 5.10. Specific wear rates of plain CF reinforced specimens under sliding wear.

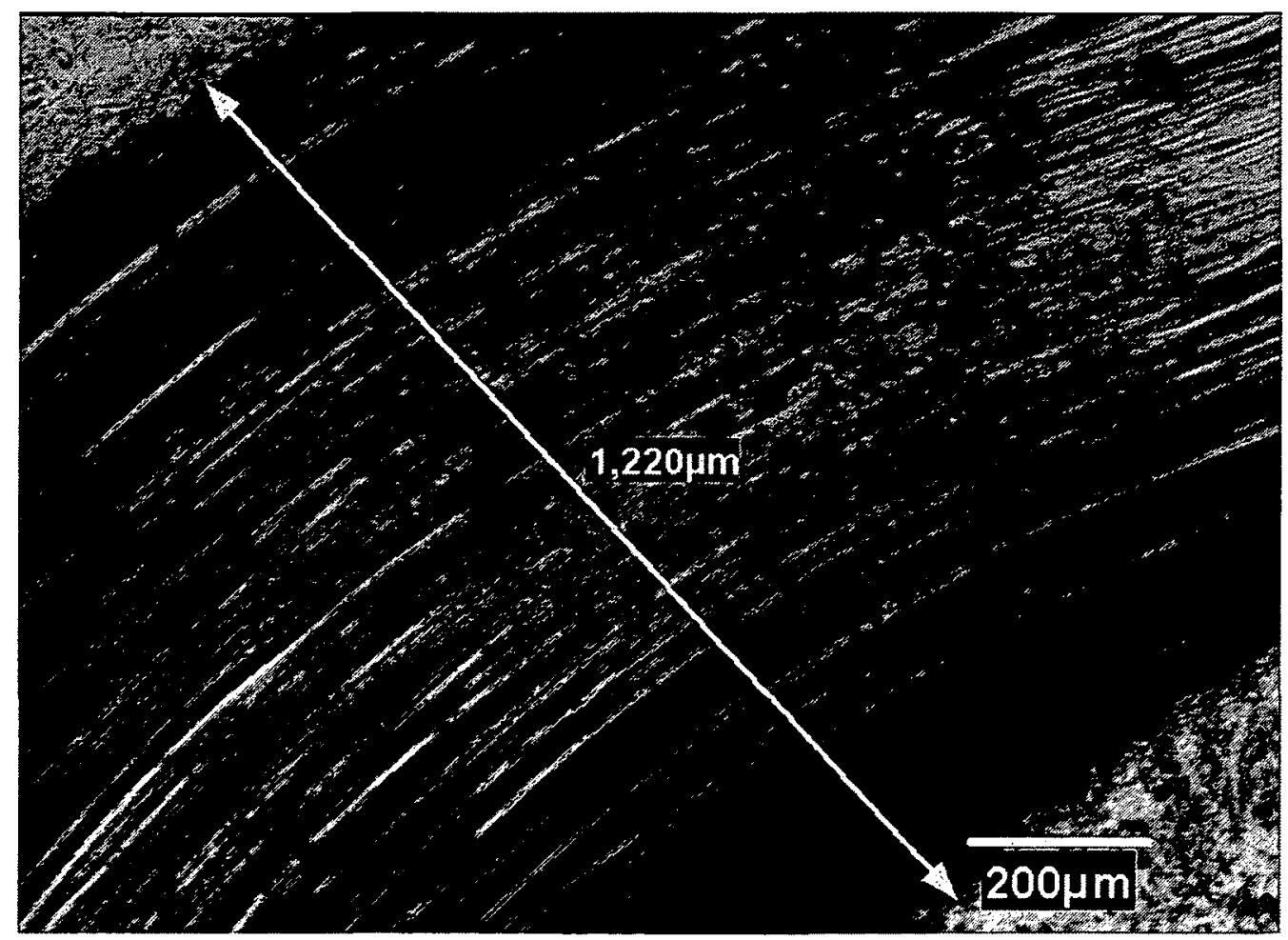

(a) 


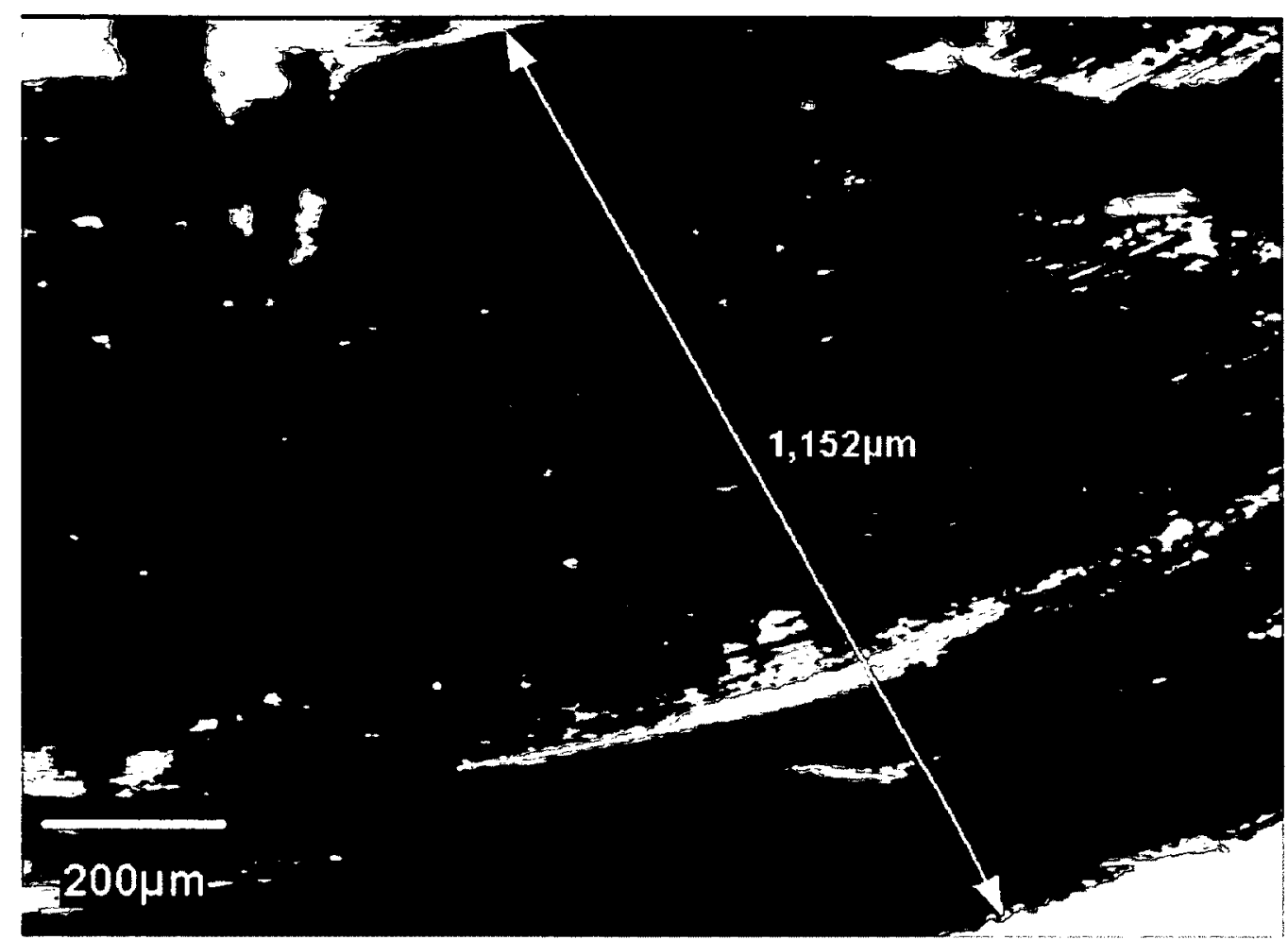

(b)

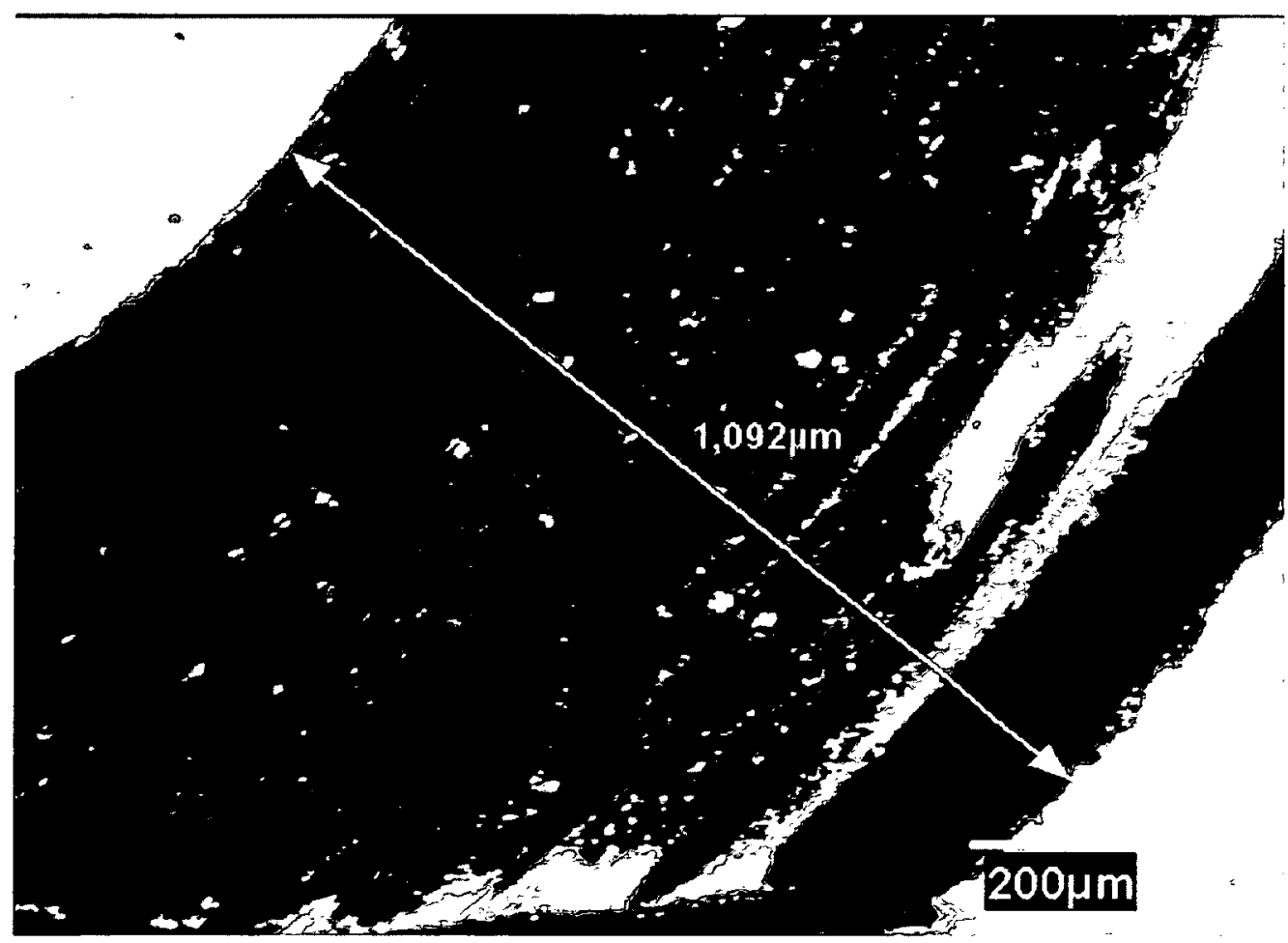

(c) 


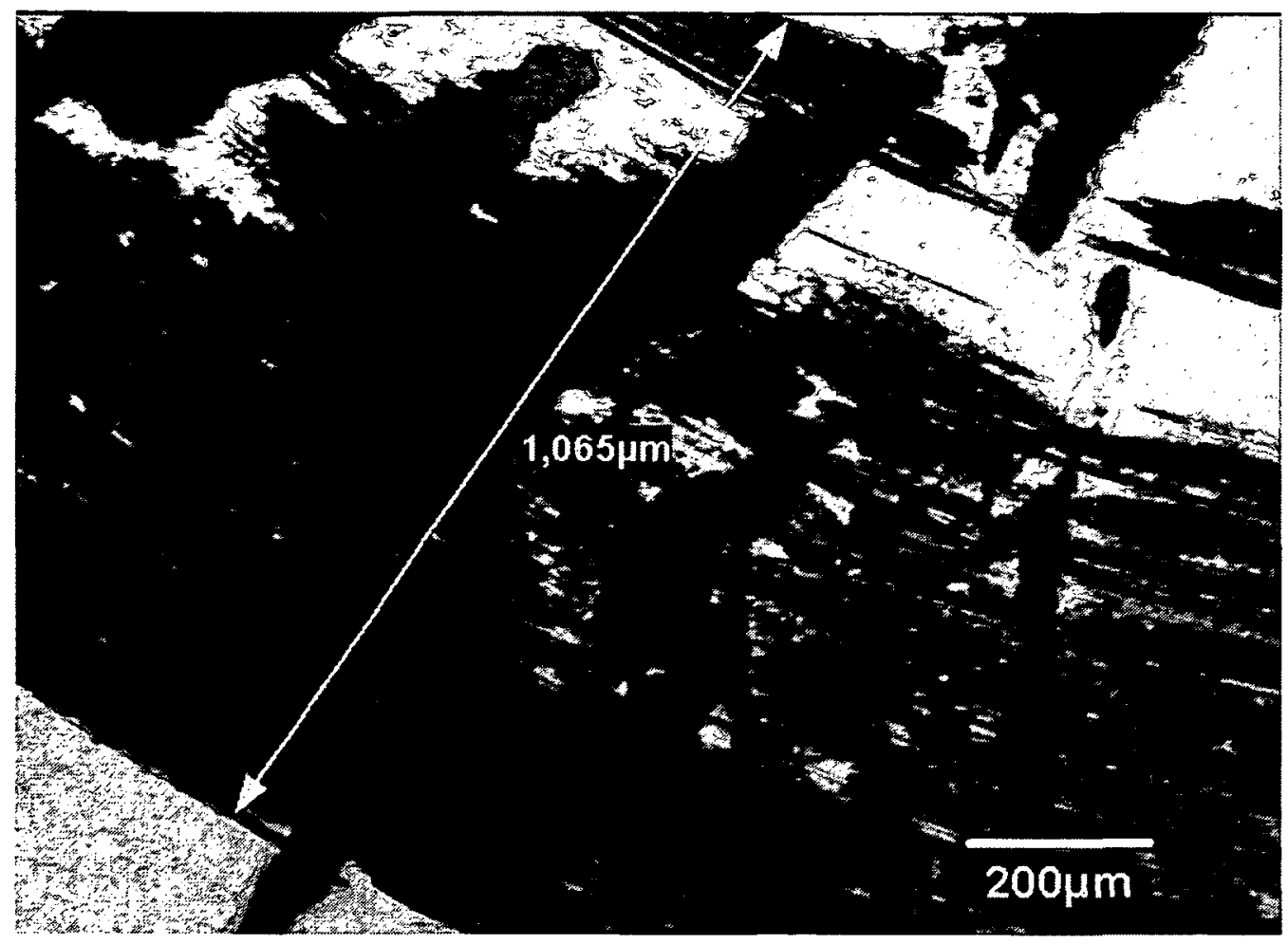

(d)

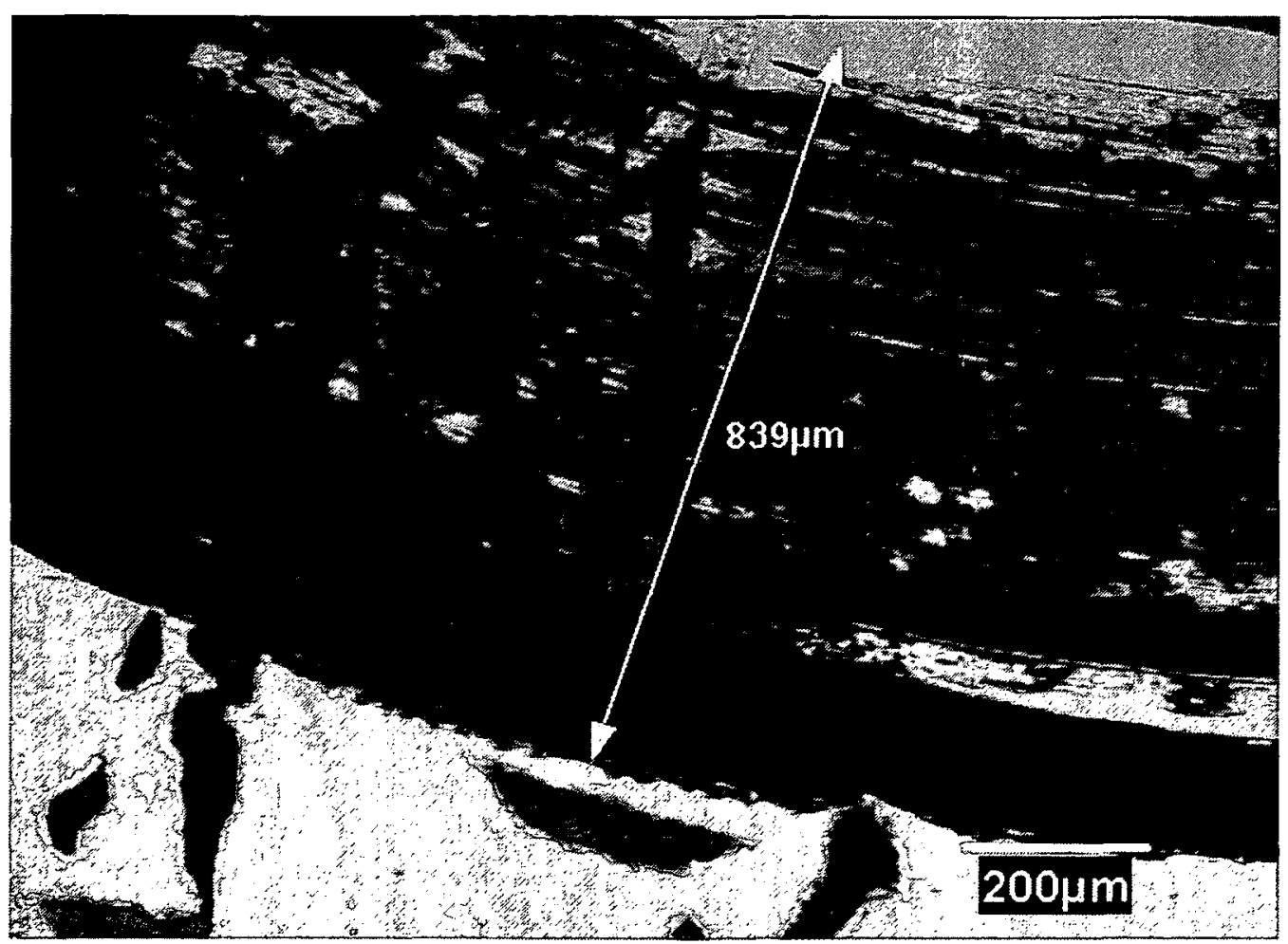

(e) 


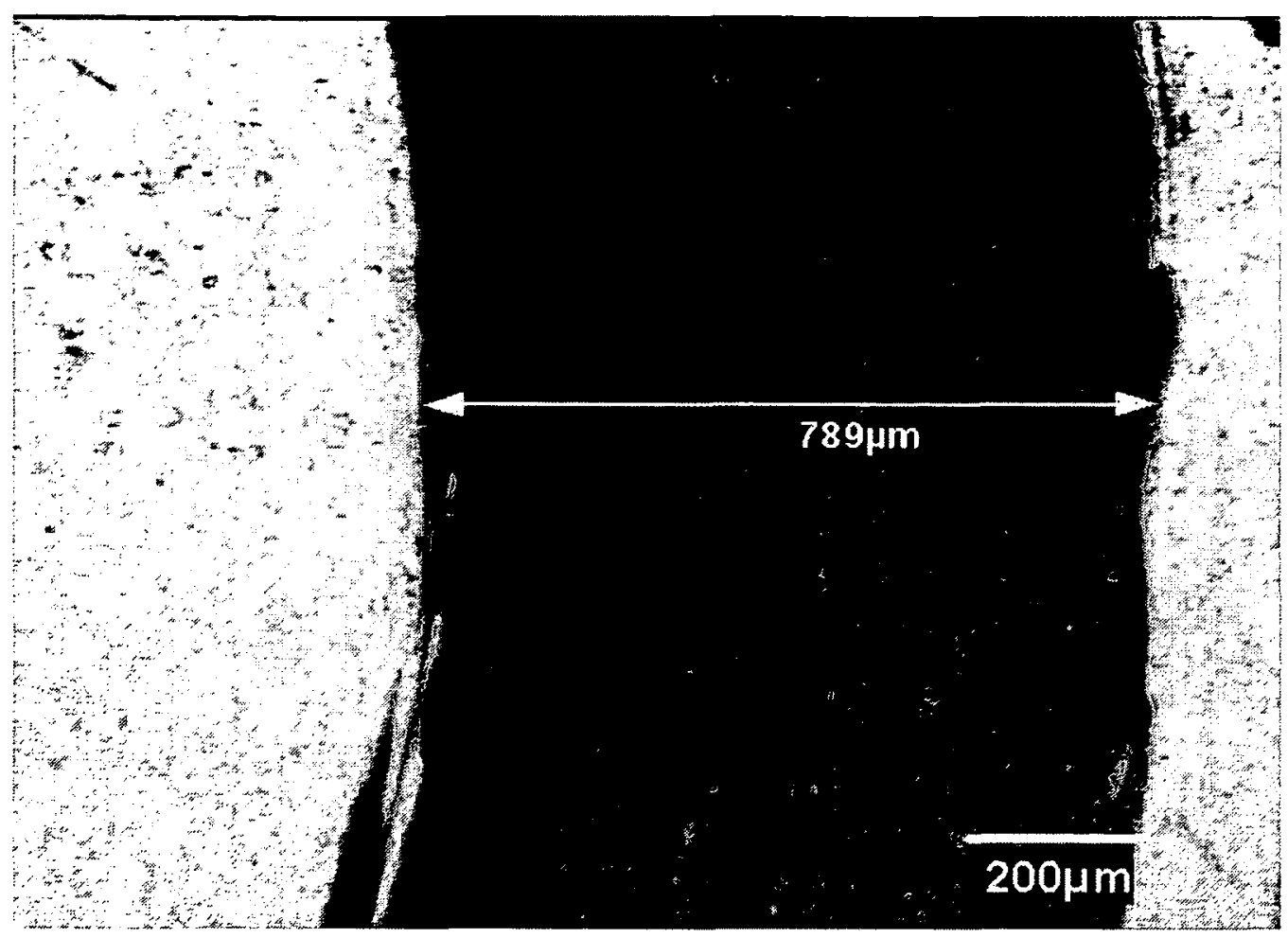

(f)

Fig. 5.11. Optical images of worn surfaces: (a) specimen A, (b) specimen B, (c) specimen C, (d) specimen D, (e) specimen $E$ and (f) specimen $F$. 


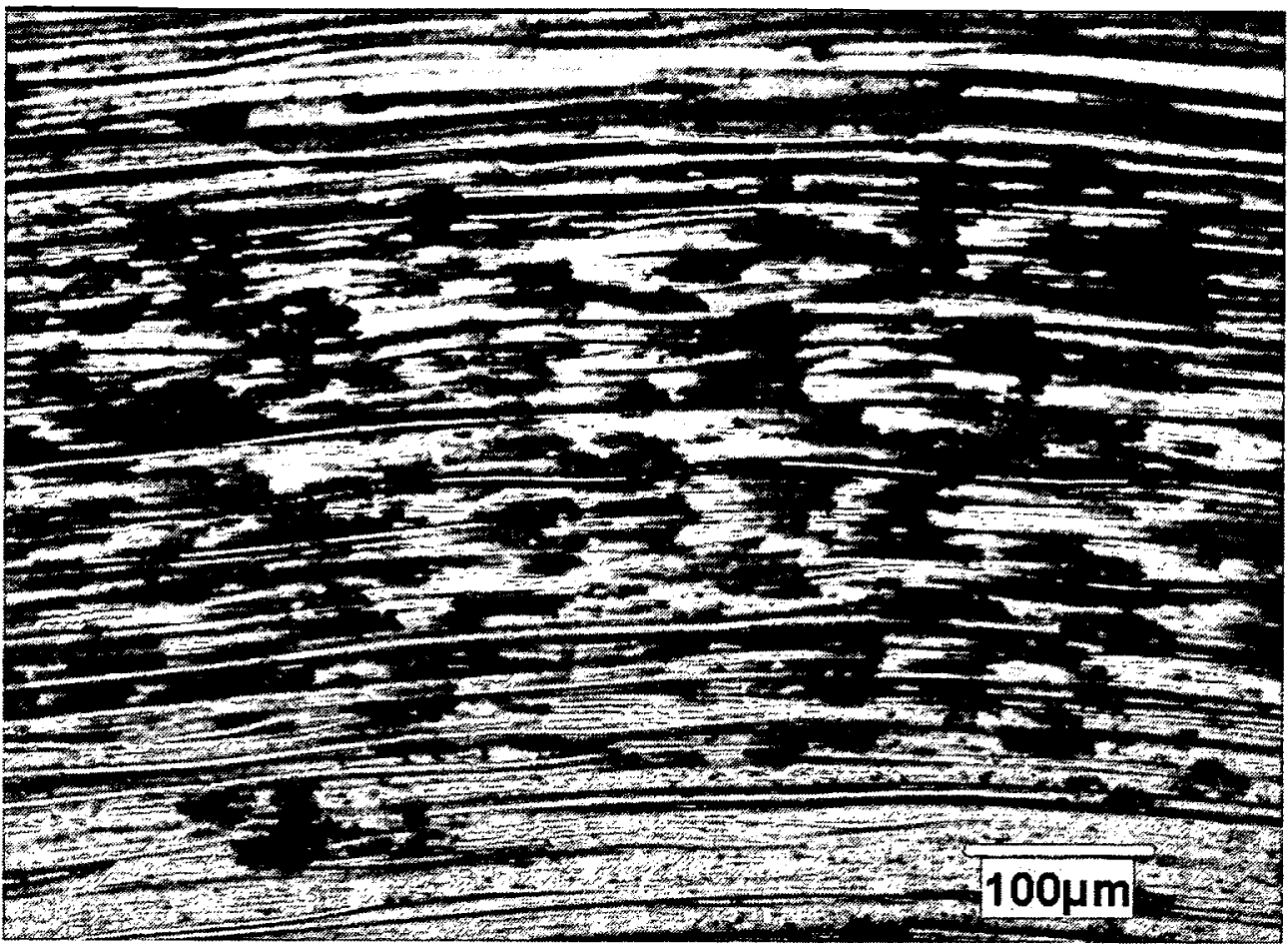

Fig. 5.12. Severe wear scars in the specimen A surface.

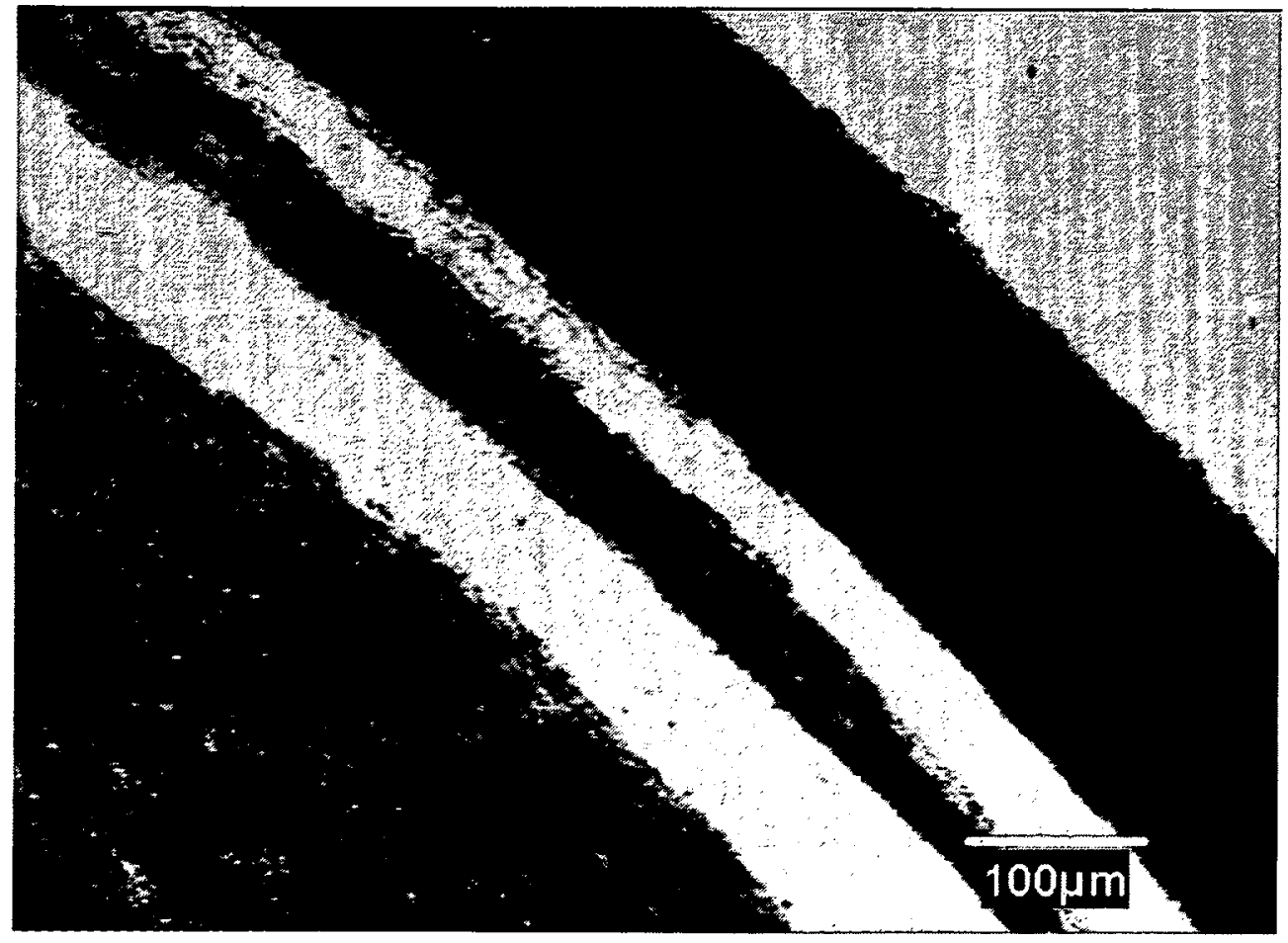

Fig. 5.13. Intermittent nature of the wear tracks in plain CF reinforced composites. 


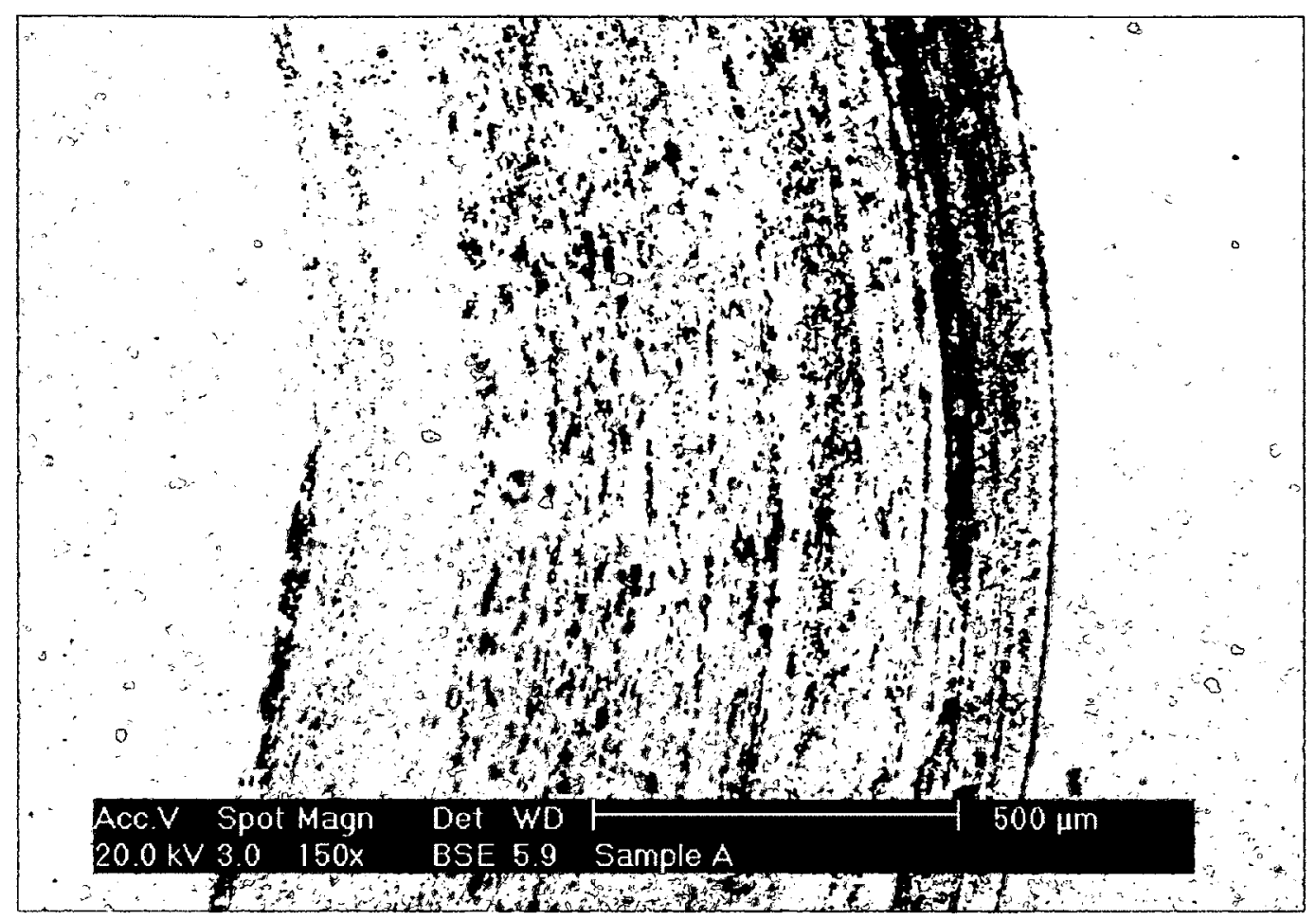

(a)

Sample A, matrix wear track

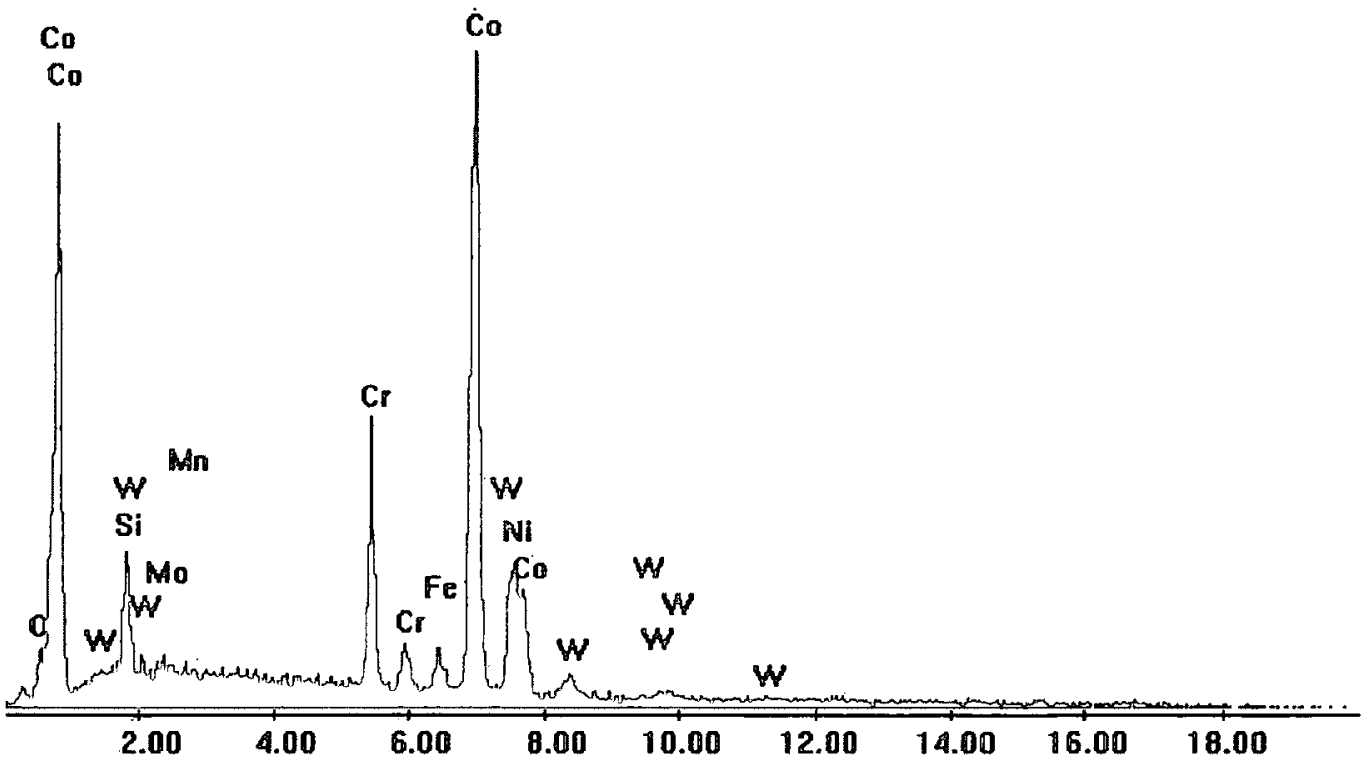

(b)

Fig. 5.14. Worn surface analyses of pure Stellite 25 specimen: (a) SEM morphology and (b) EDX spectrum. 


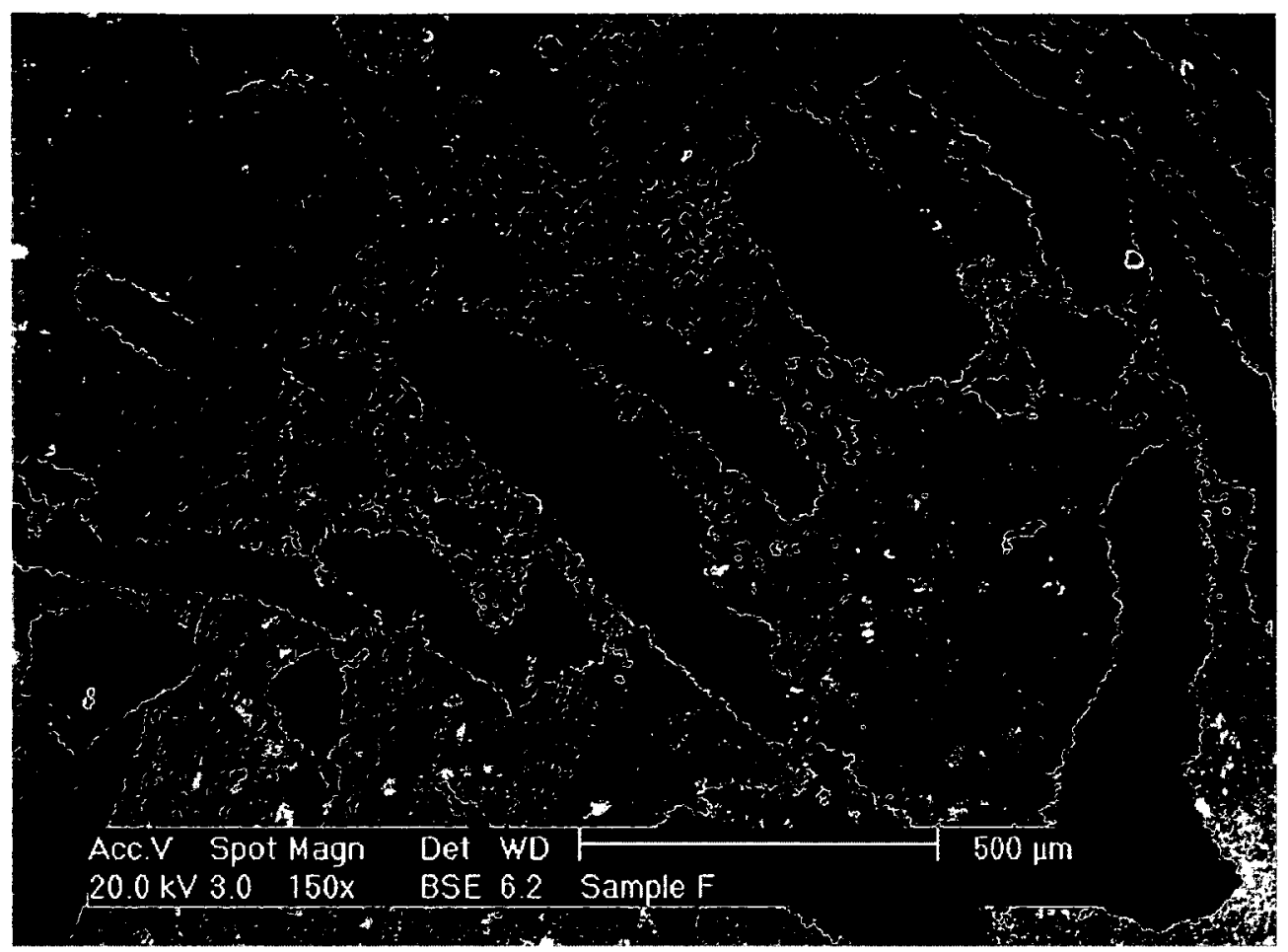

(a)

Sample F, wear track

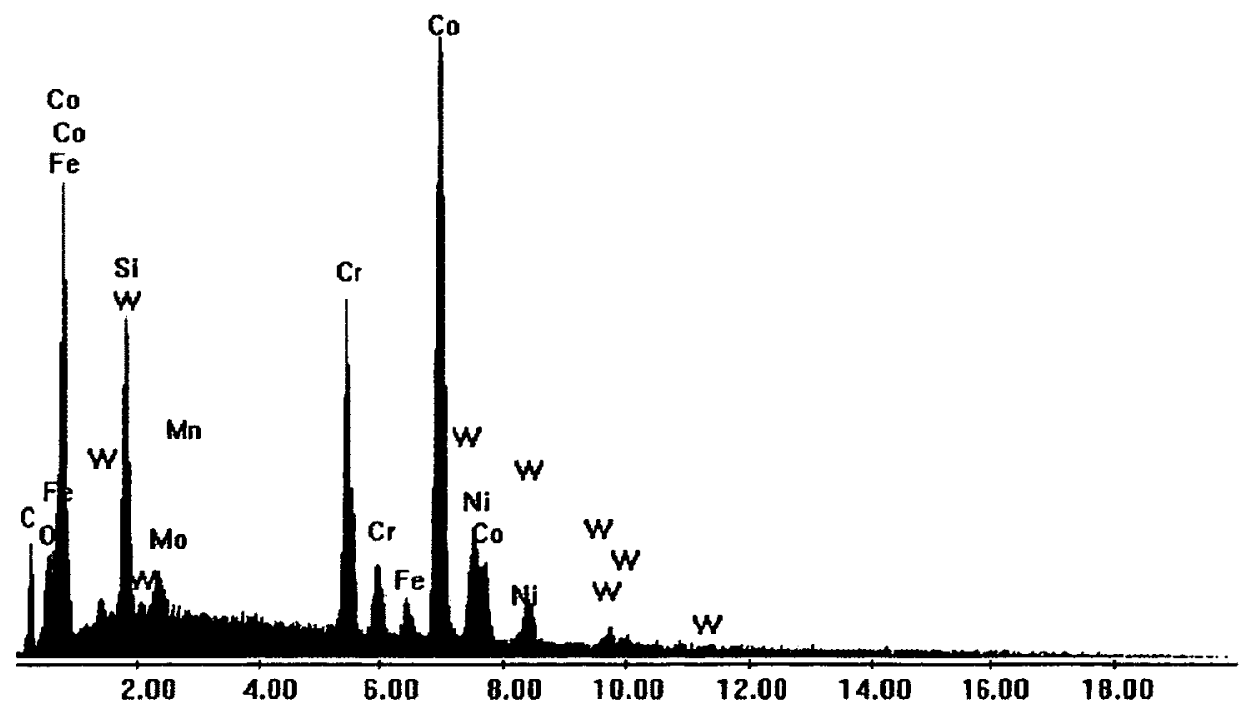

(b)

Fig. 5.15. Worn surface analyses of composite specimen F: (a) SEM morphology and (b) EDX spectrum. 


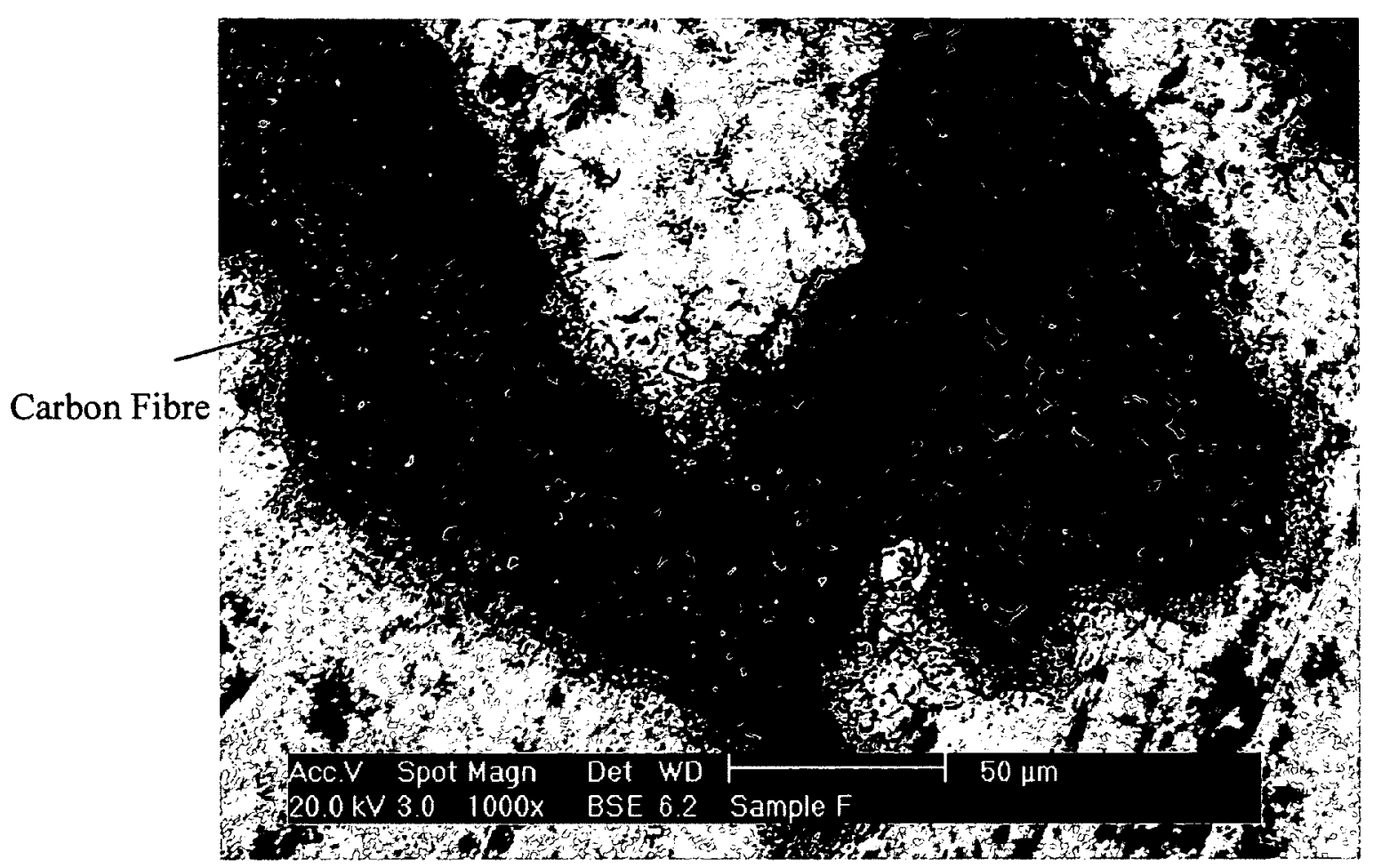

(a)

Sample F, wear track $\mathrm{C}$ fiber

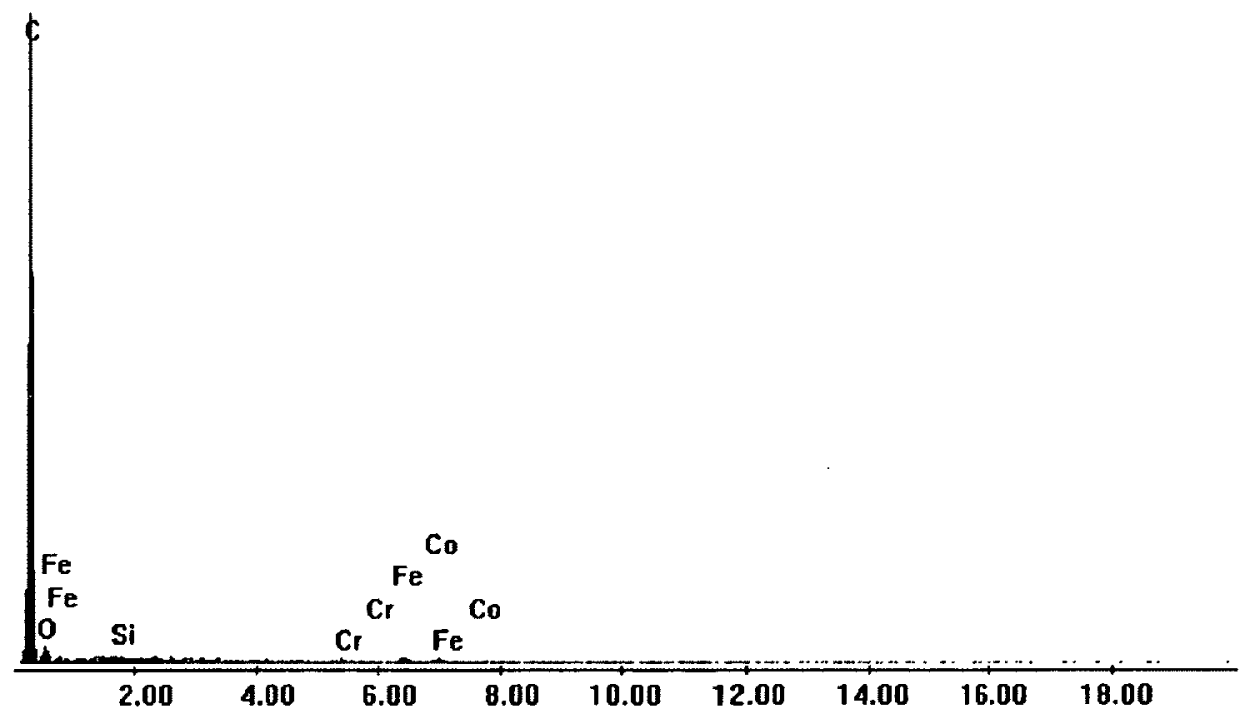

(b)

Fig. 5.16. Worn surface analyses of carbon fibres in composite specimen F: (a) SEM morphology and (b) EDX spectrum. 


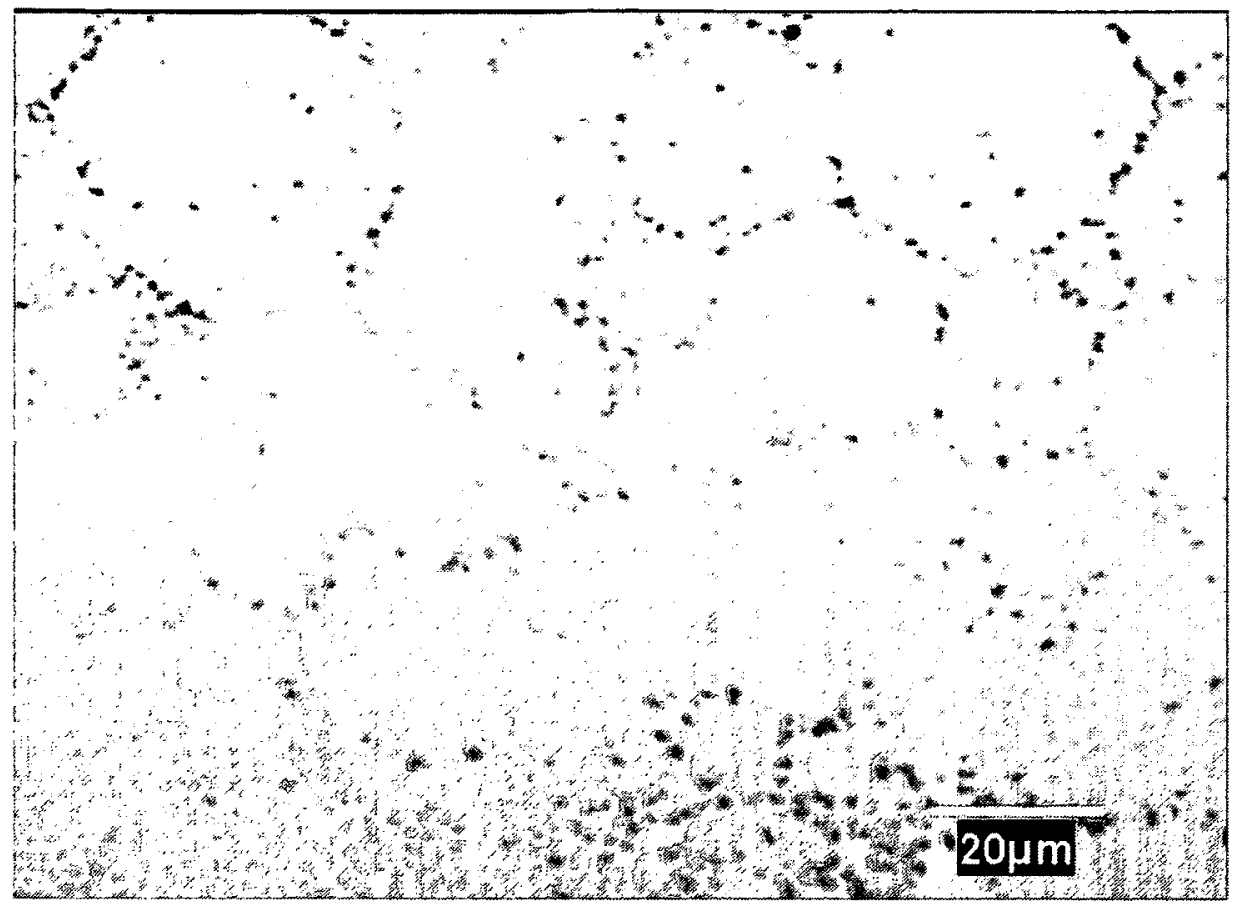

(a)

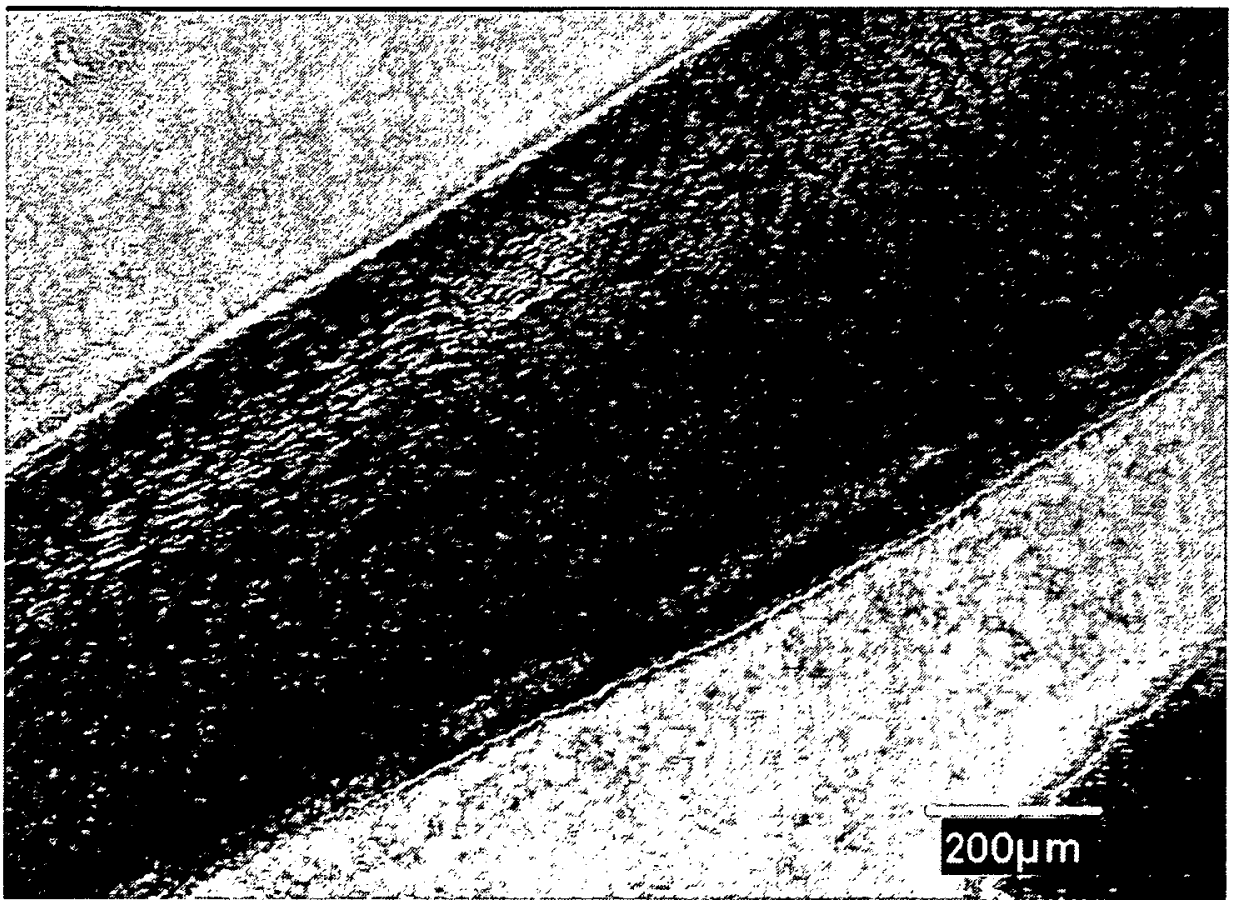

(b) 


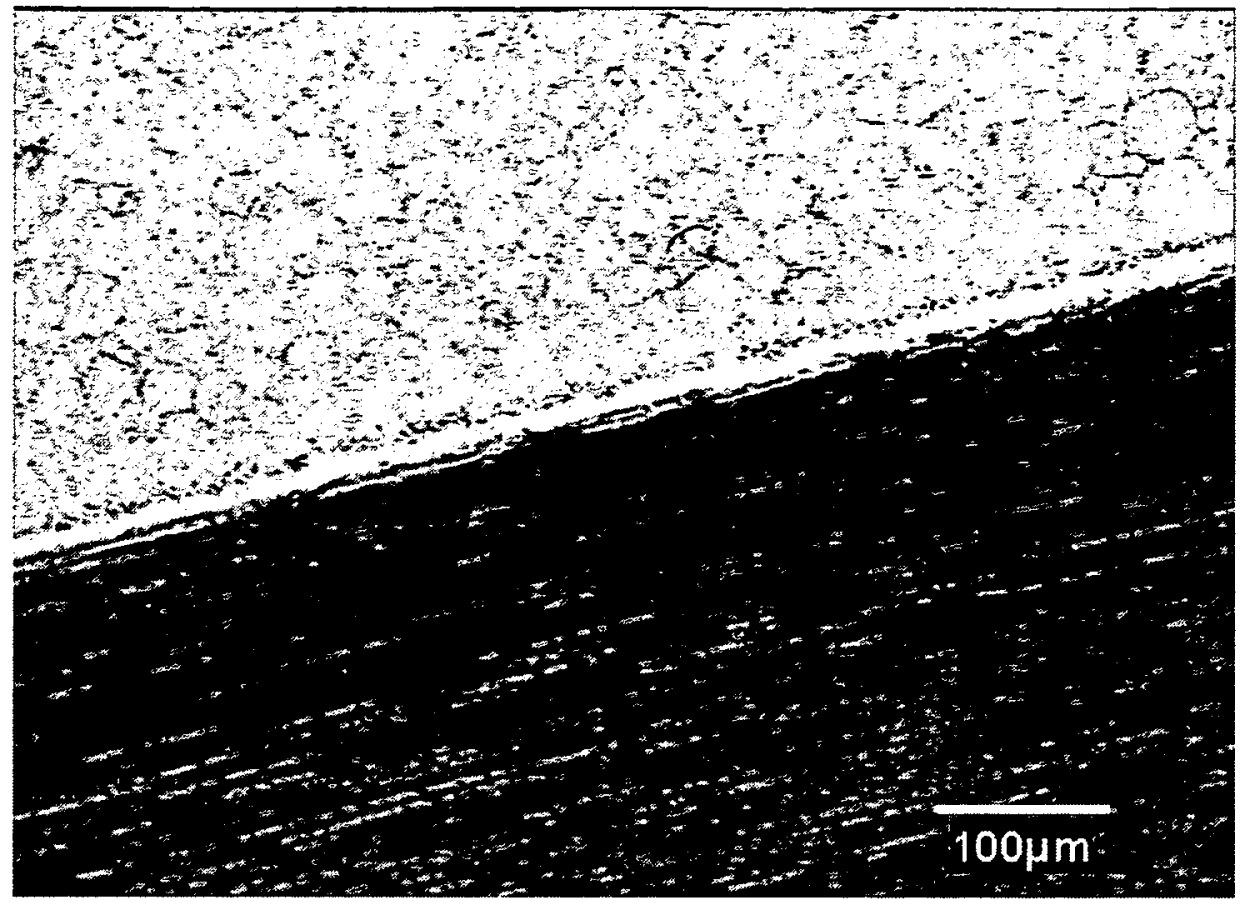

(c)

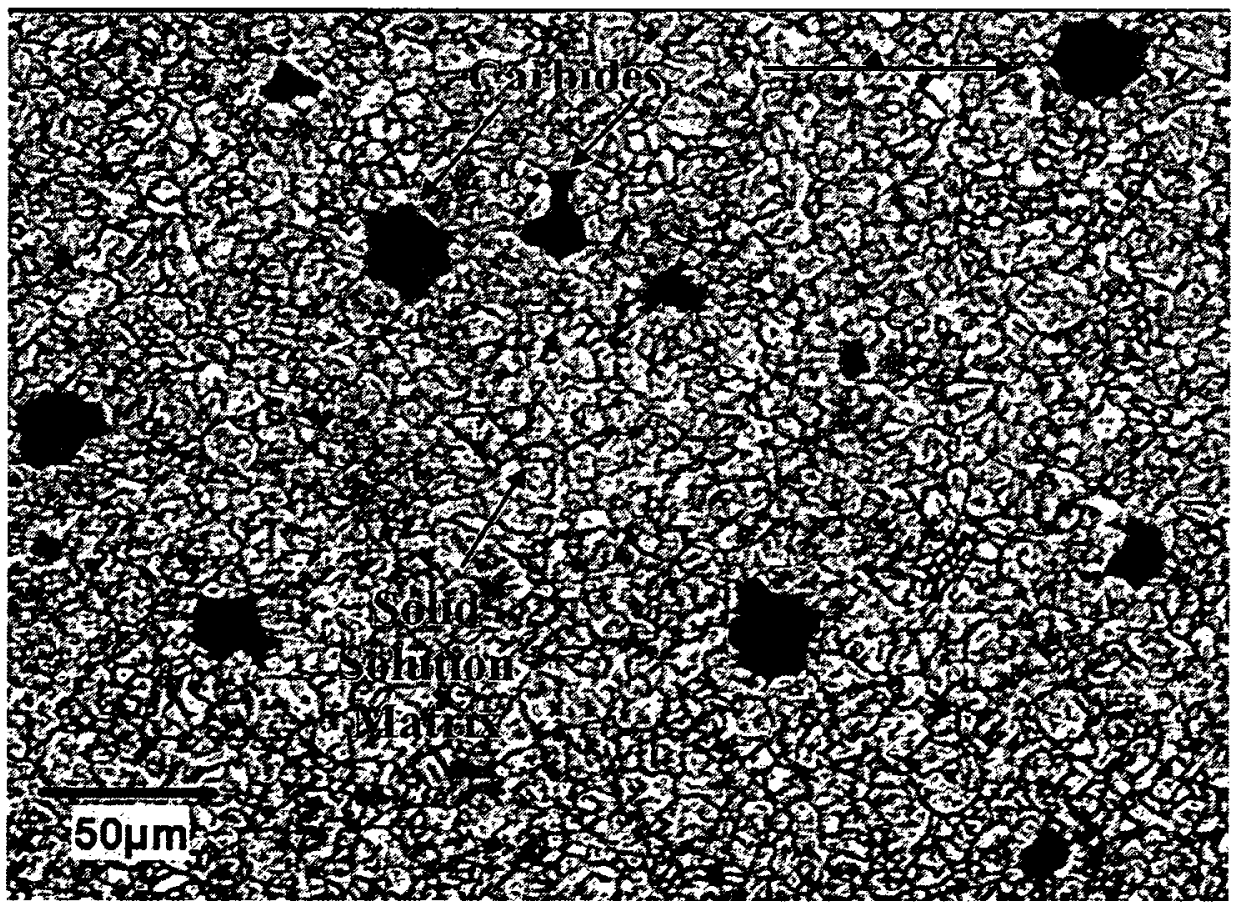

(d)

Fig. 5.17. Microstructural images: (a) Unetched pure Stellite 25 (specimen A), (b) unetched Nicoated CF reinforced composite (specimen $P$ ), (c) unetched specimen $P$ at higher magnification and (d) the matrix of etched specimen $P$ in an area close to the fibres. 


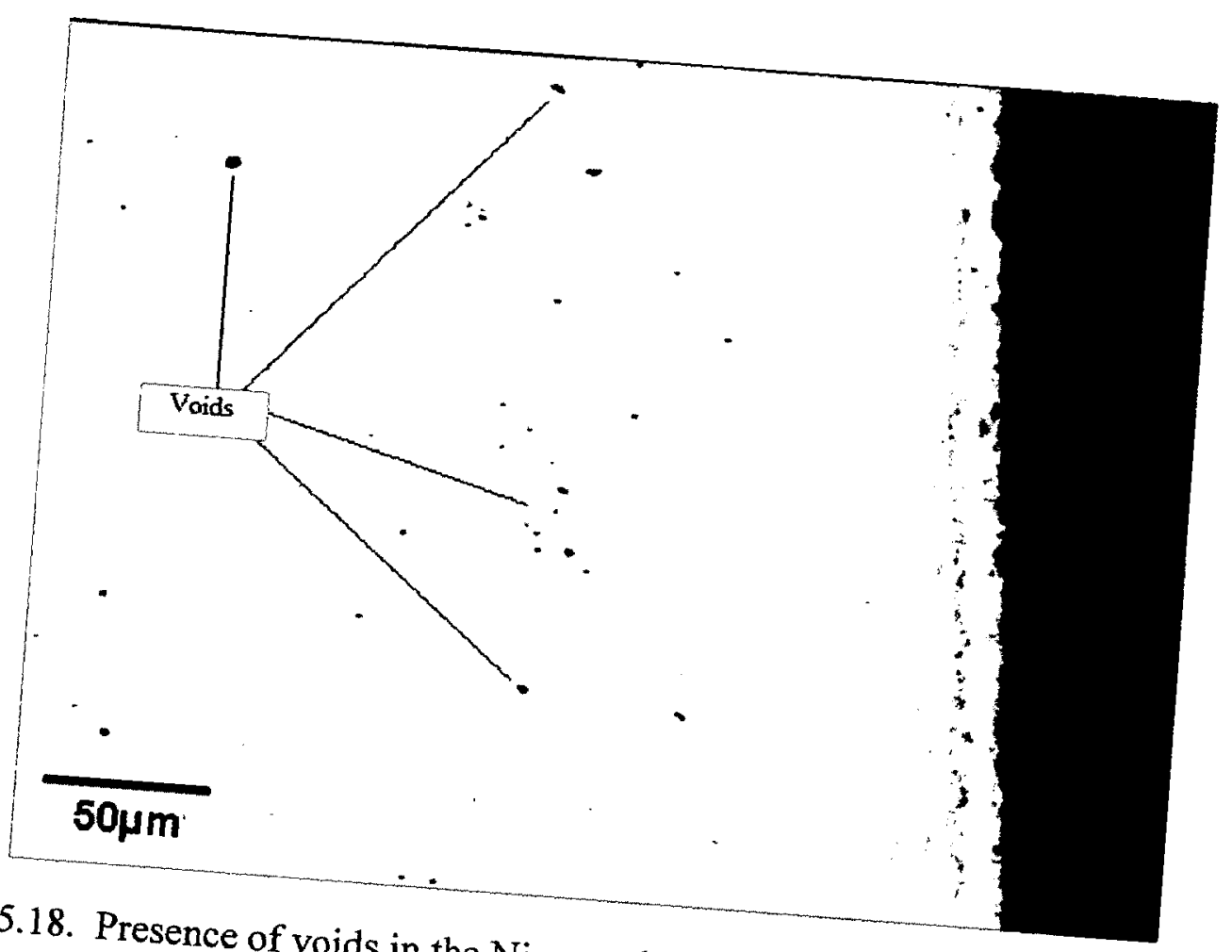

Fig. 5.18. Presence of voids in the Ni-coated carbon fibre reinforced composites.

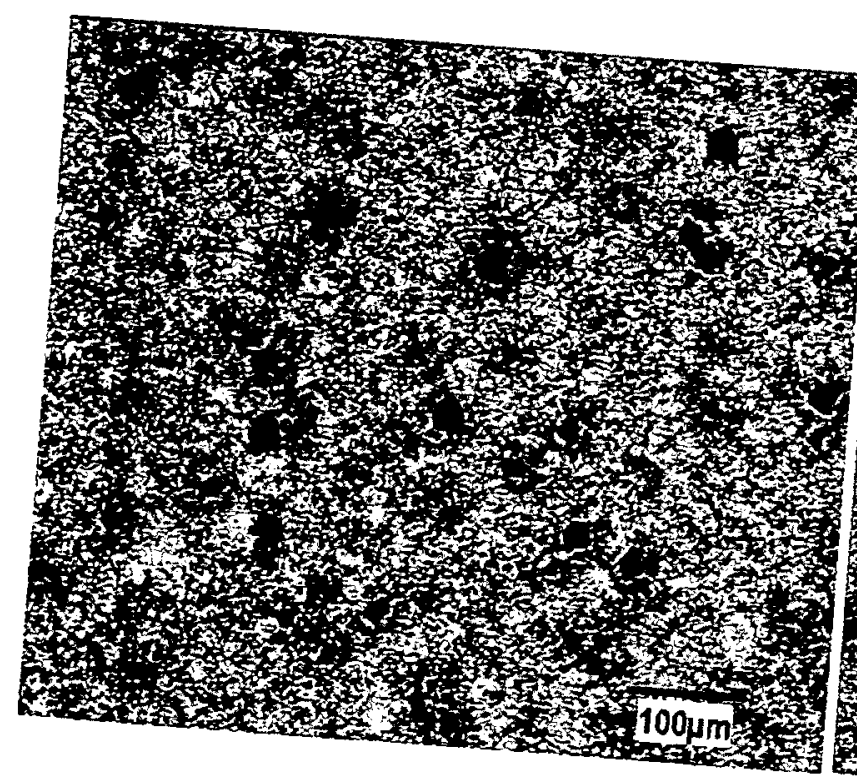

(a)

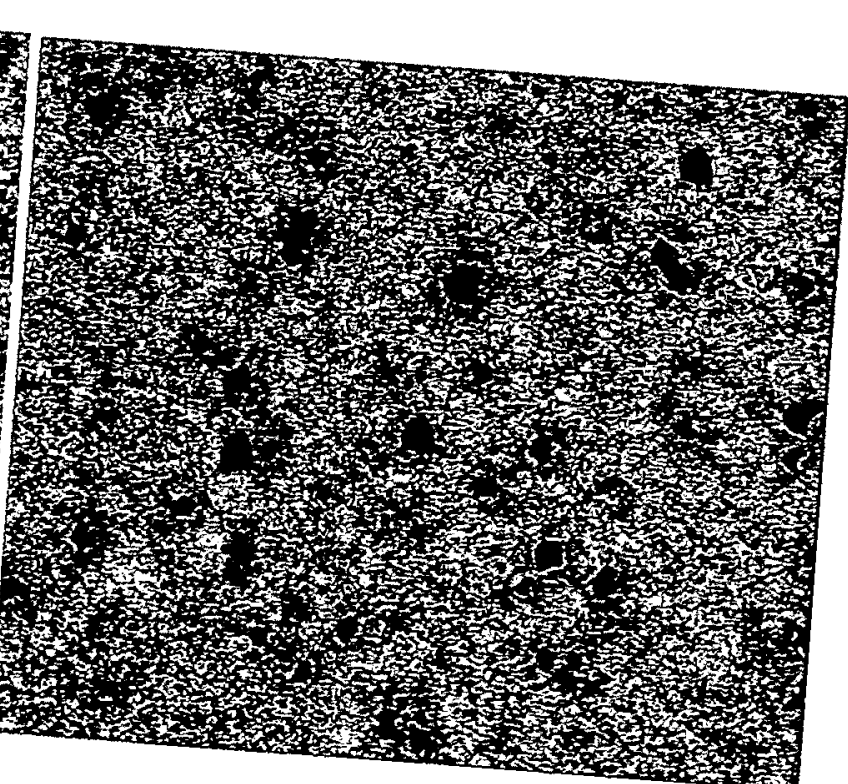




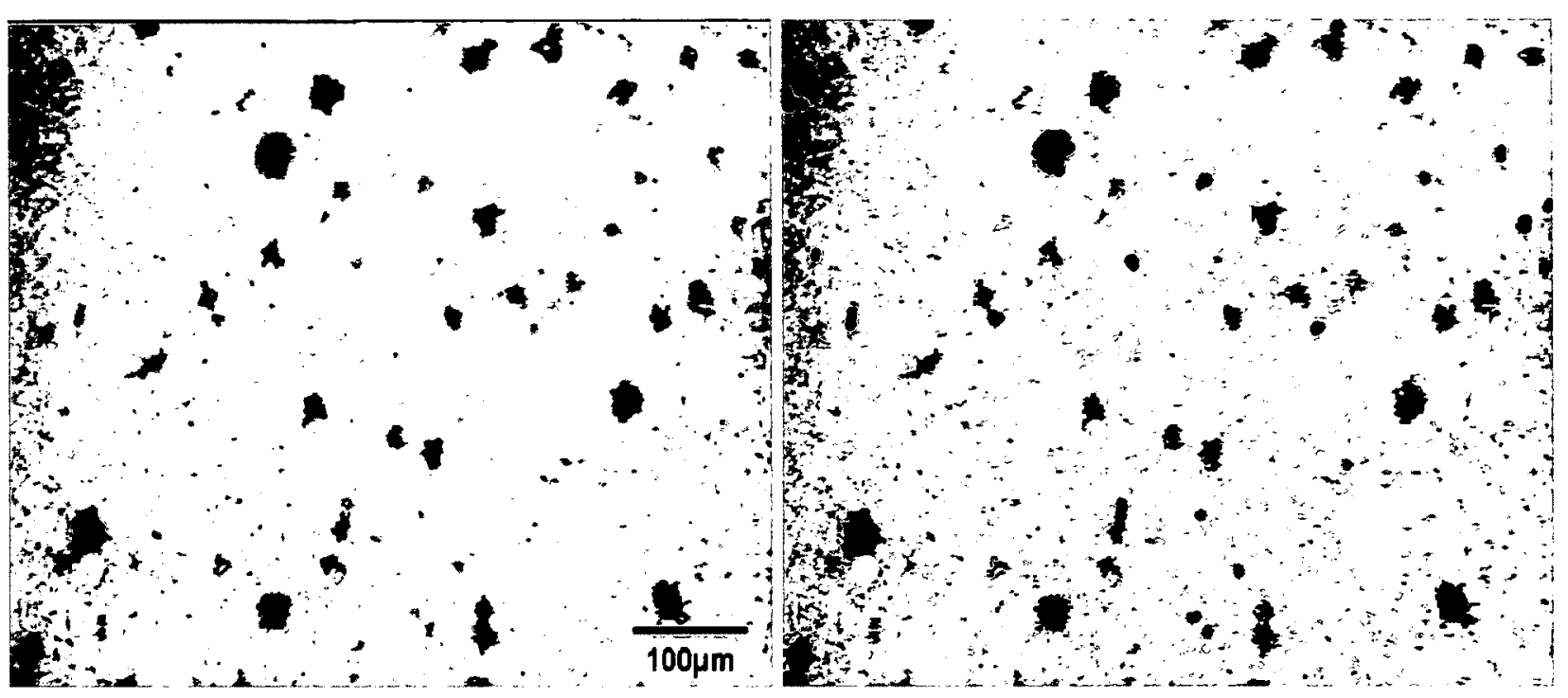

(b)
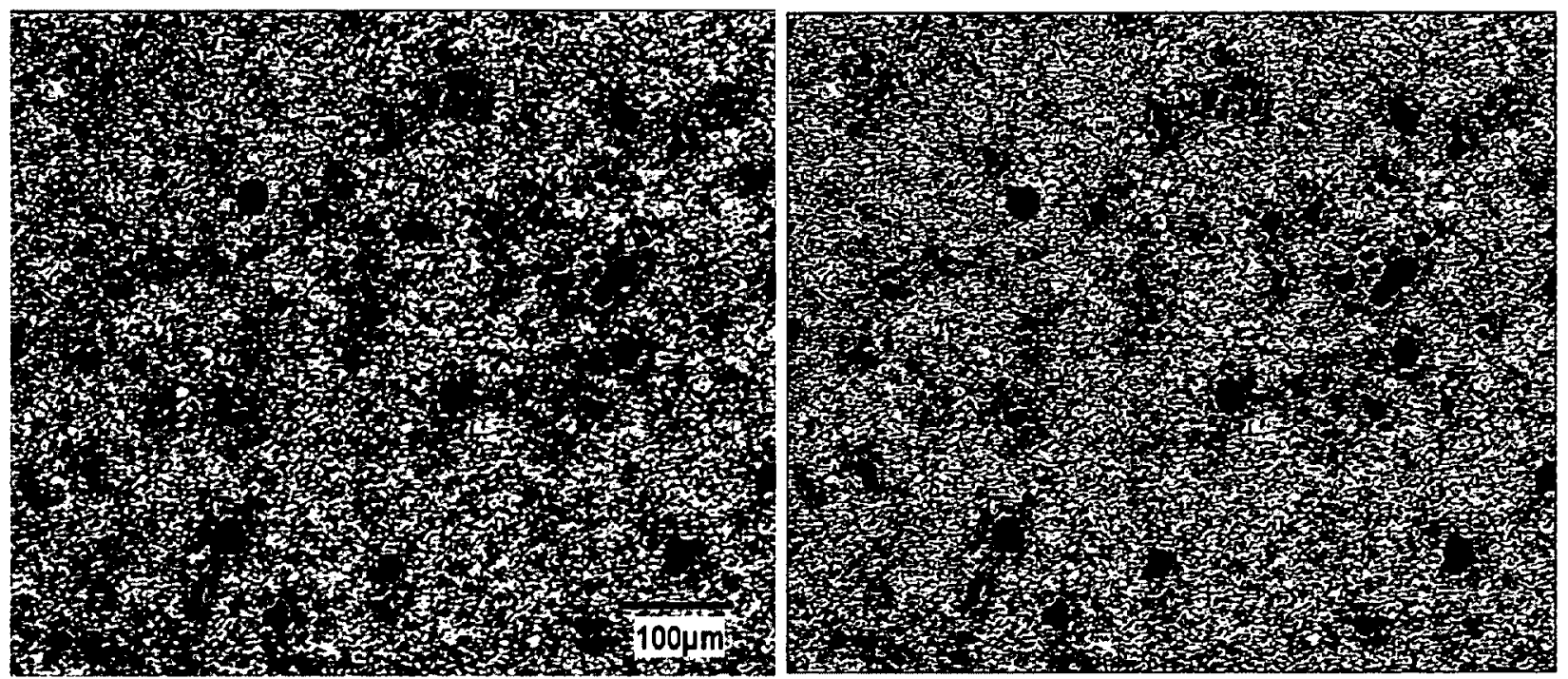

(c) 


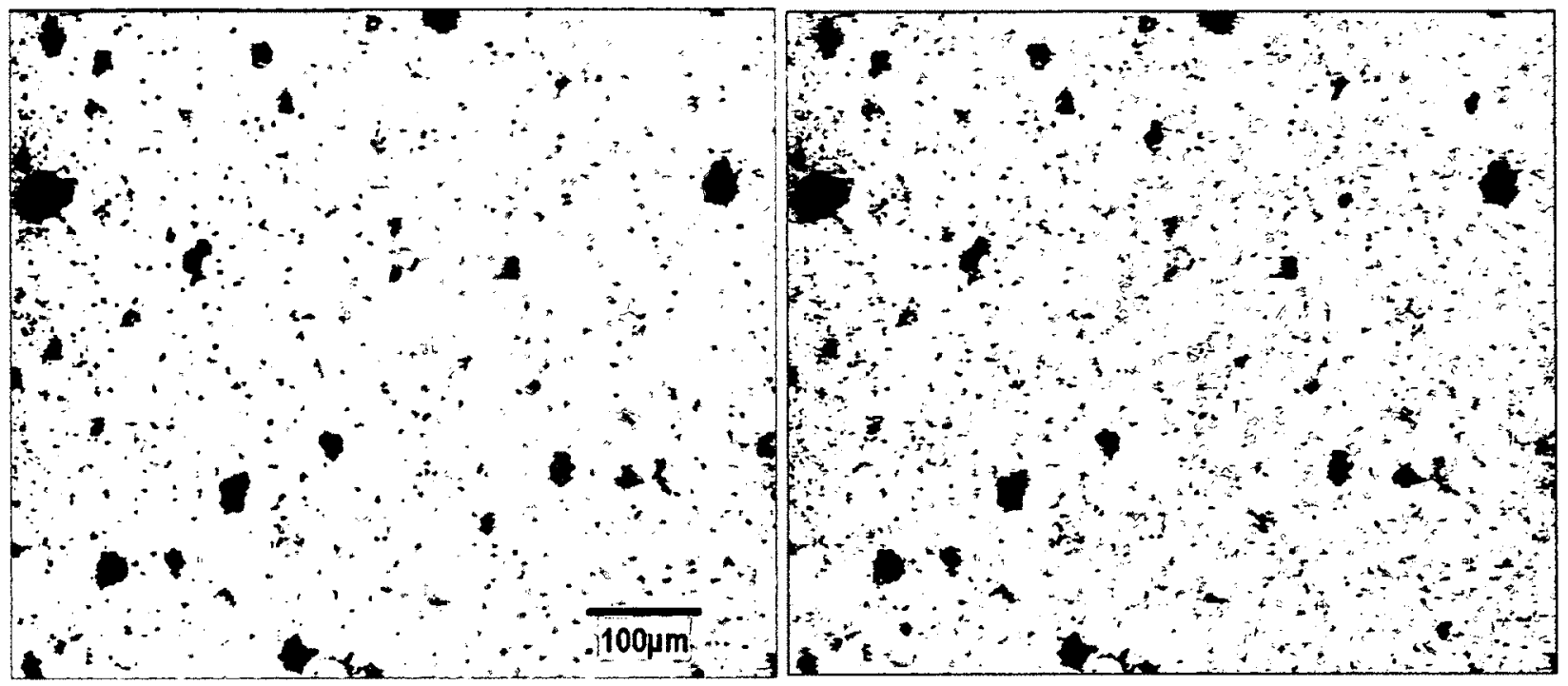

(d)

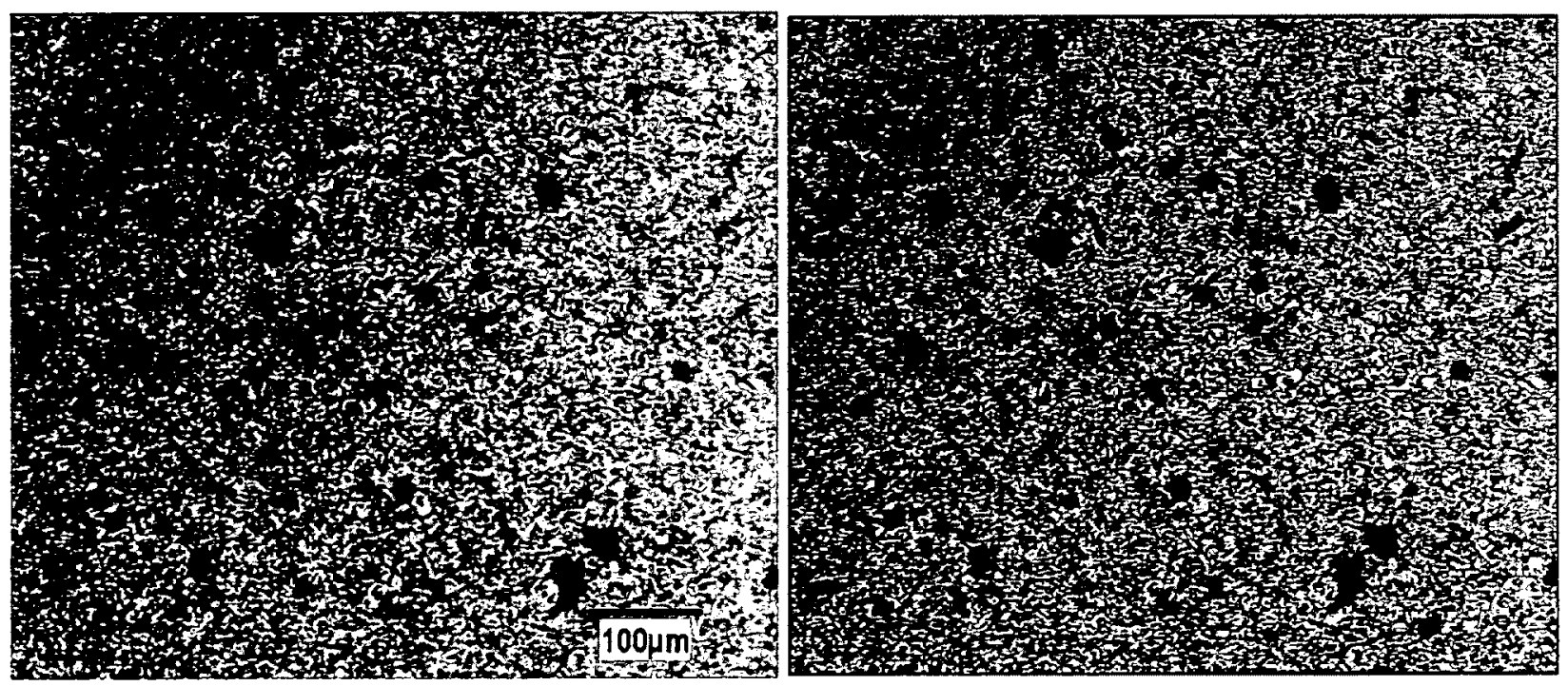

(e) 

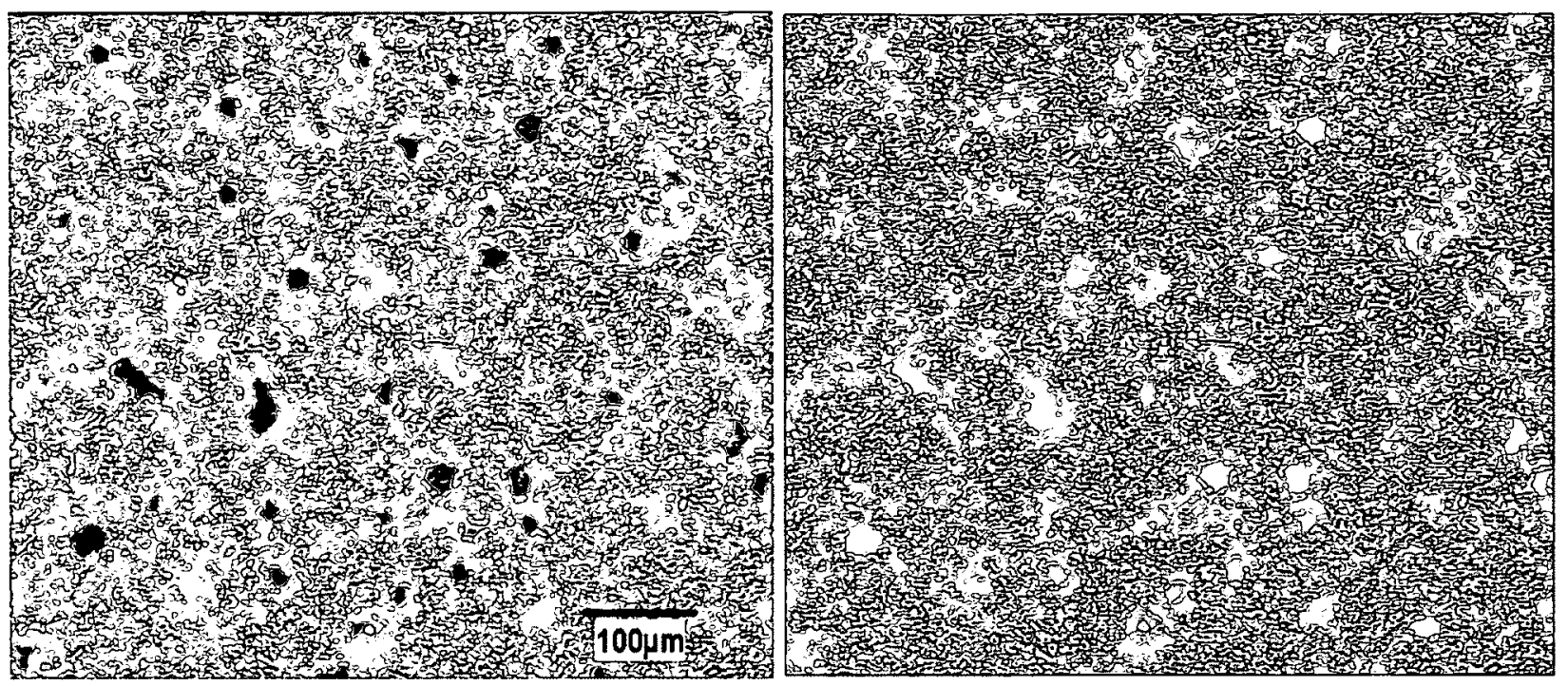

(f)
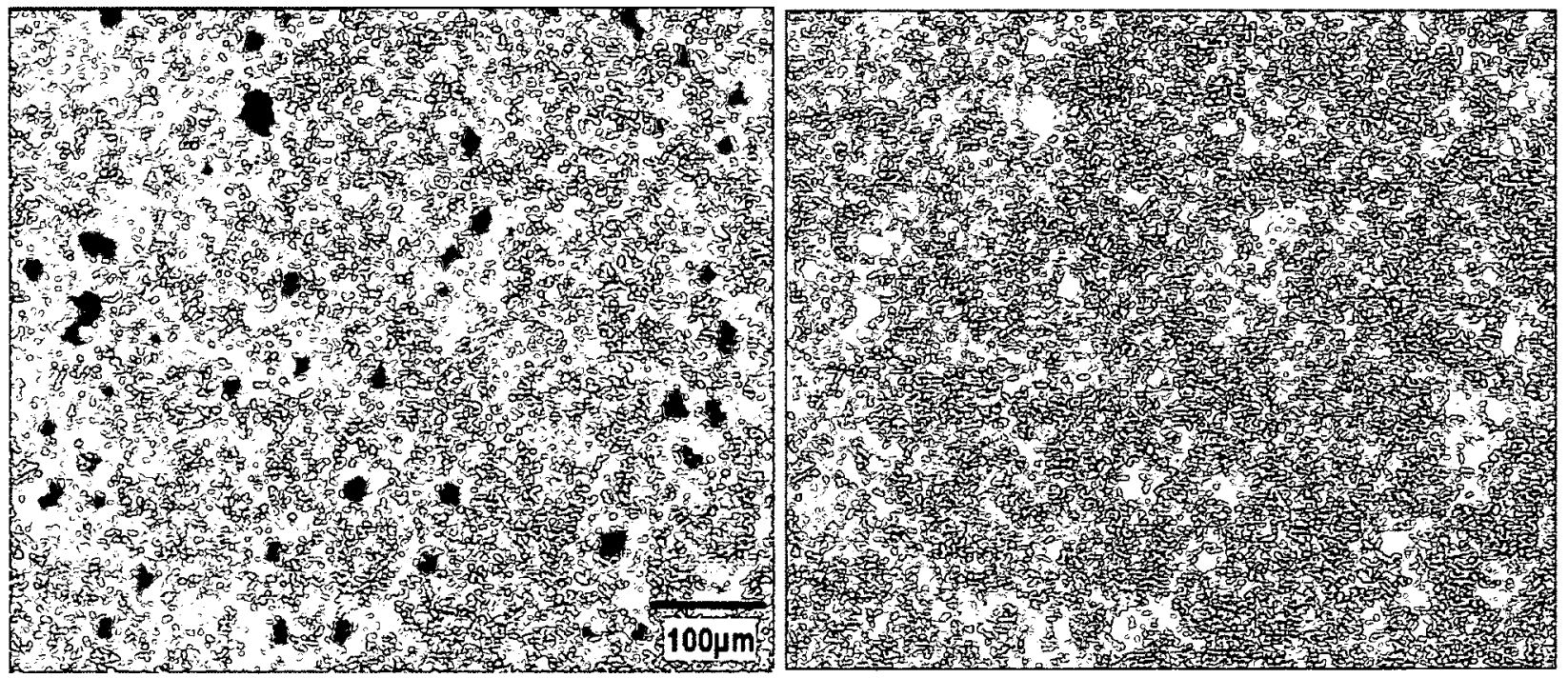

(g)

Fig. 5.19. Pictures used for carbide volume fraction analysis: (a) specimen A, (b) specimen K, (c) specimen L, (d) specimen $M$, (e) specimen $N$, (f) specimen $O$ and (g) specimen $P$. 


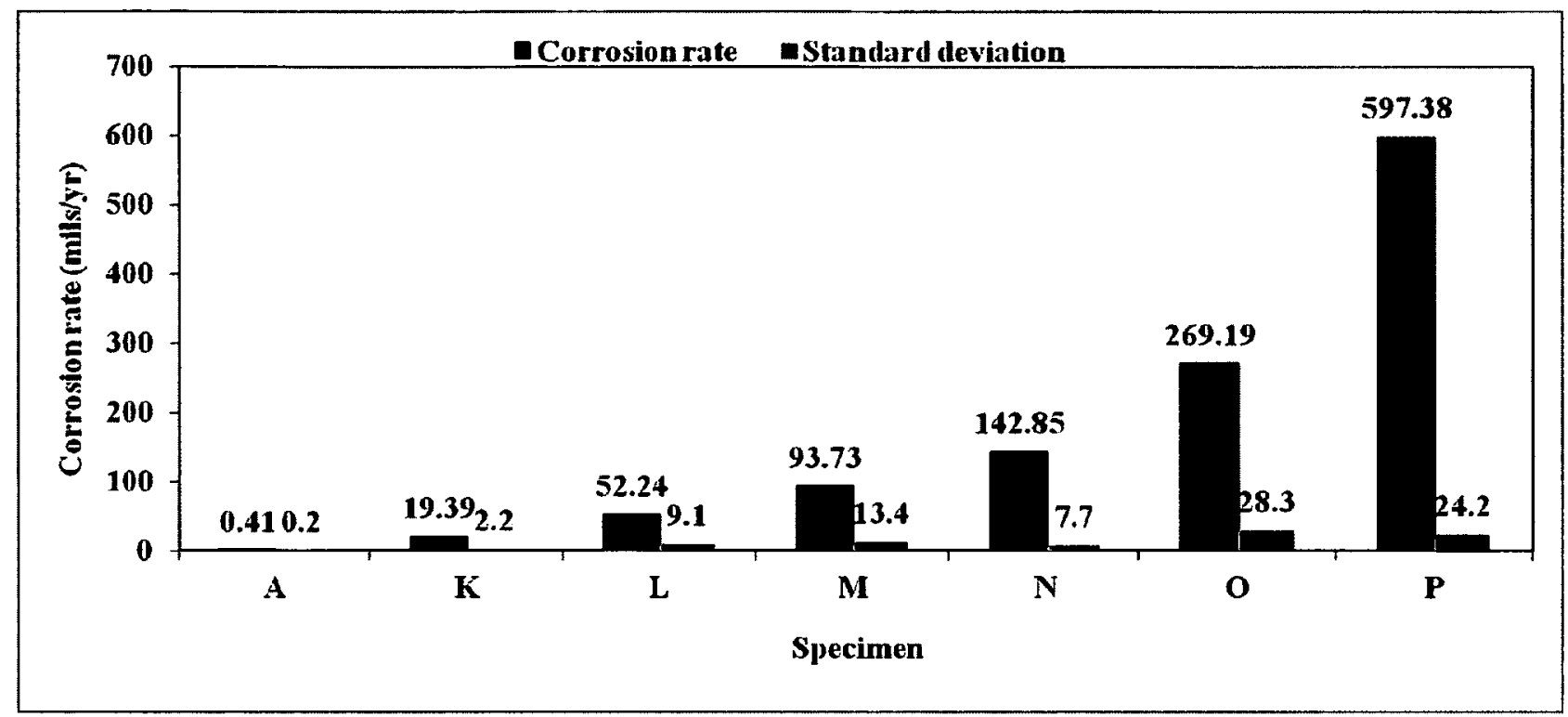

Fig. 5.20. Corrosion test results of Ni-coated CF reinforced composite specimens.

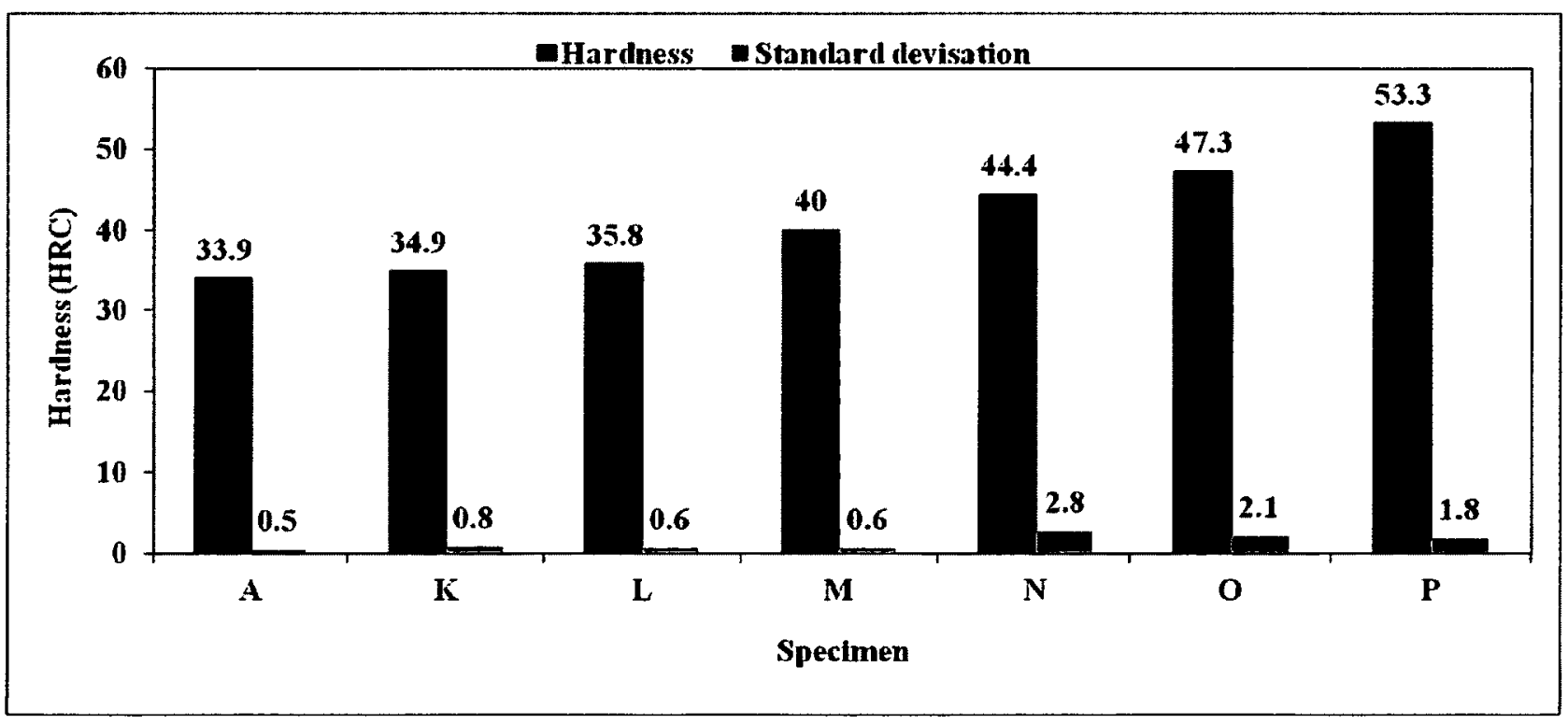

Fig. 5.21. Rockwell hardness test results of the Ni-coated carbon fibre reinforced composite specimens. 


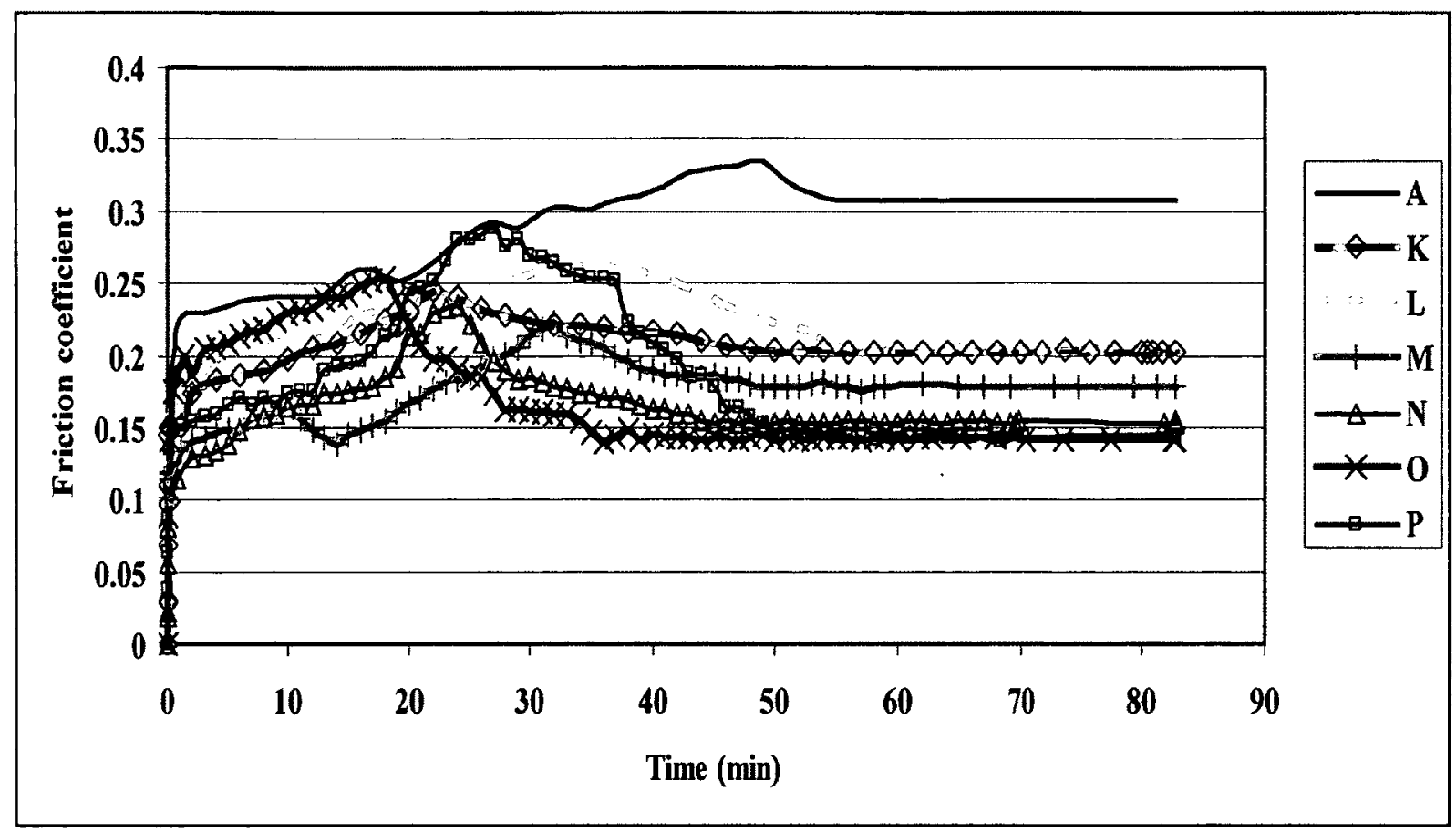

Fig. 5.22. Variations of friction coefficient for Ni-coated carbon fibre composite specimens with sliding time.

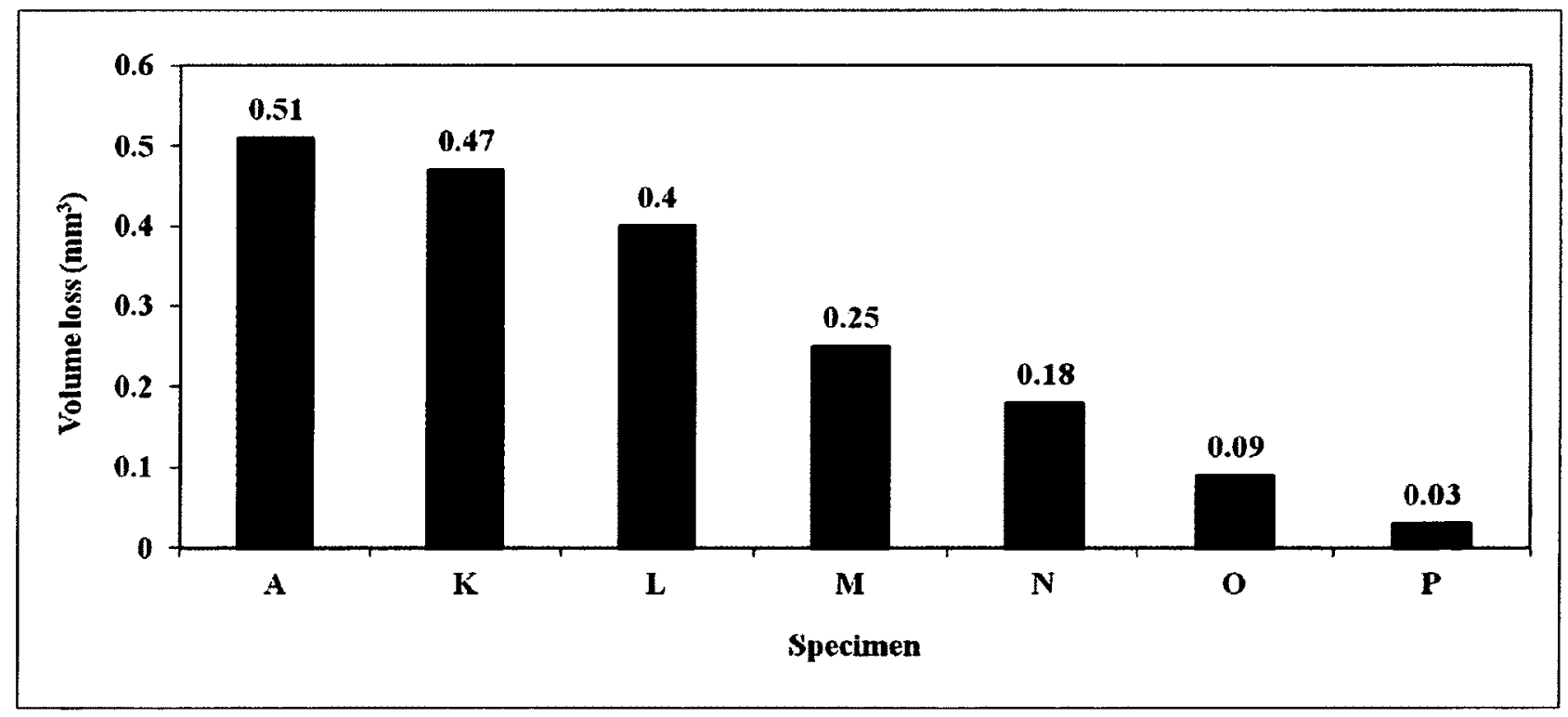

Fig. 5.23. Volume losses of $\mathrm{Ni}$-coated carbon fibre reinforced composite specimens under pin-on-disc wear. 


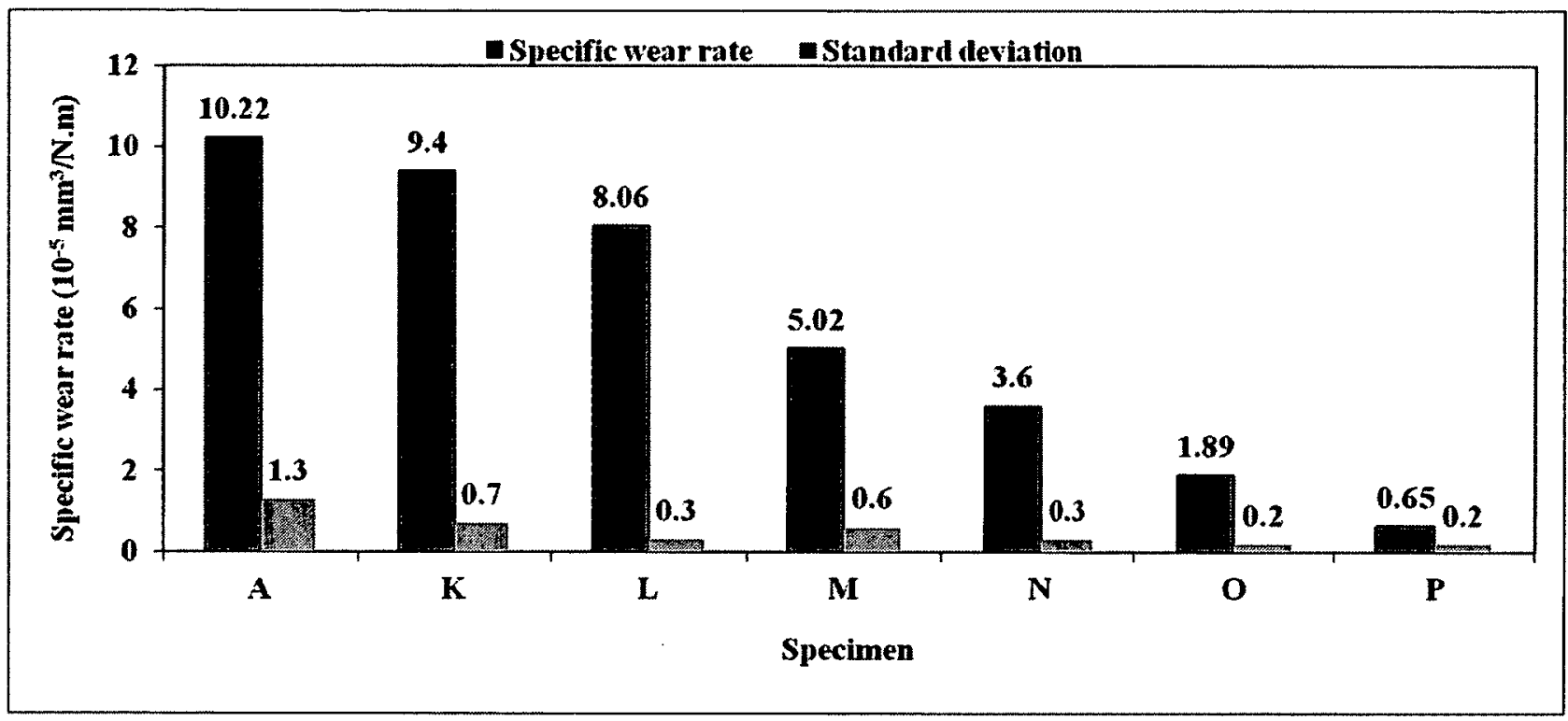

Fig. 5.24. Specific wear rates of Ni-coated carbon fibre reinforced composite specimens under pin-on-disc wear. 


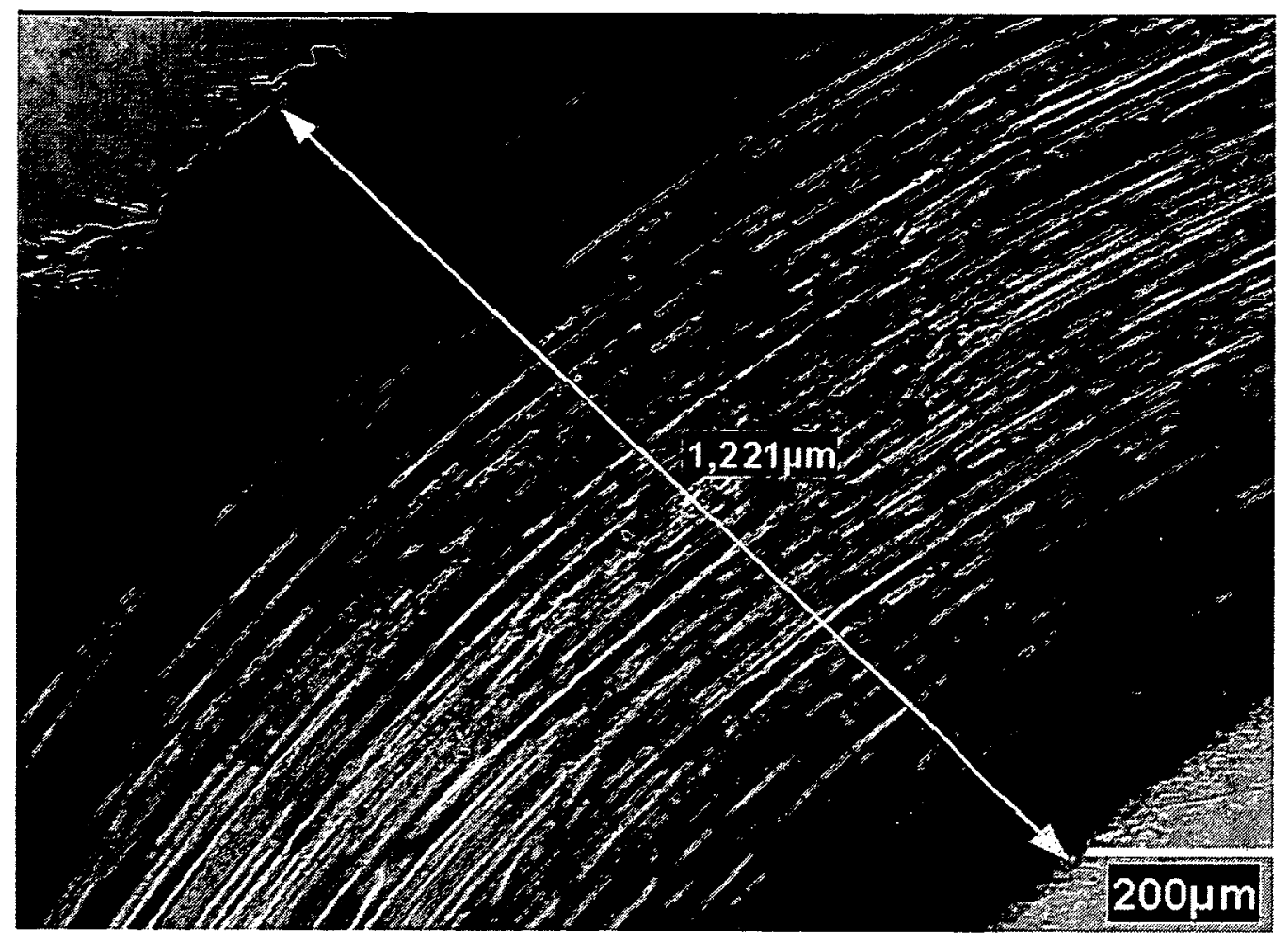

(a)

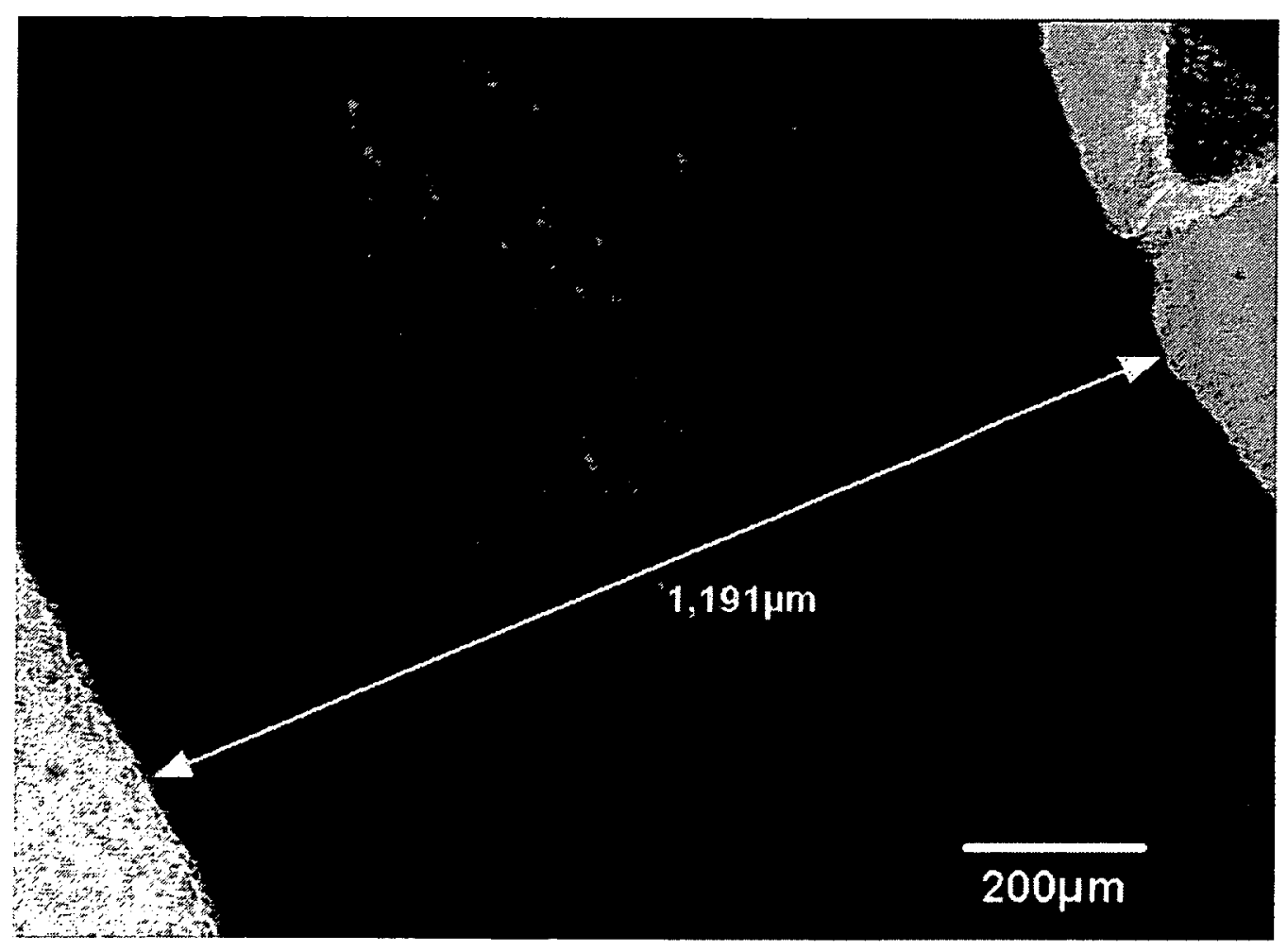

(b) 


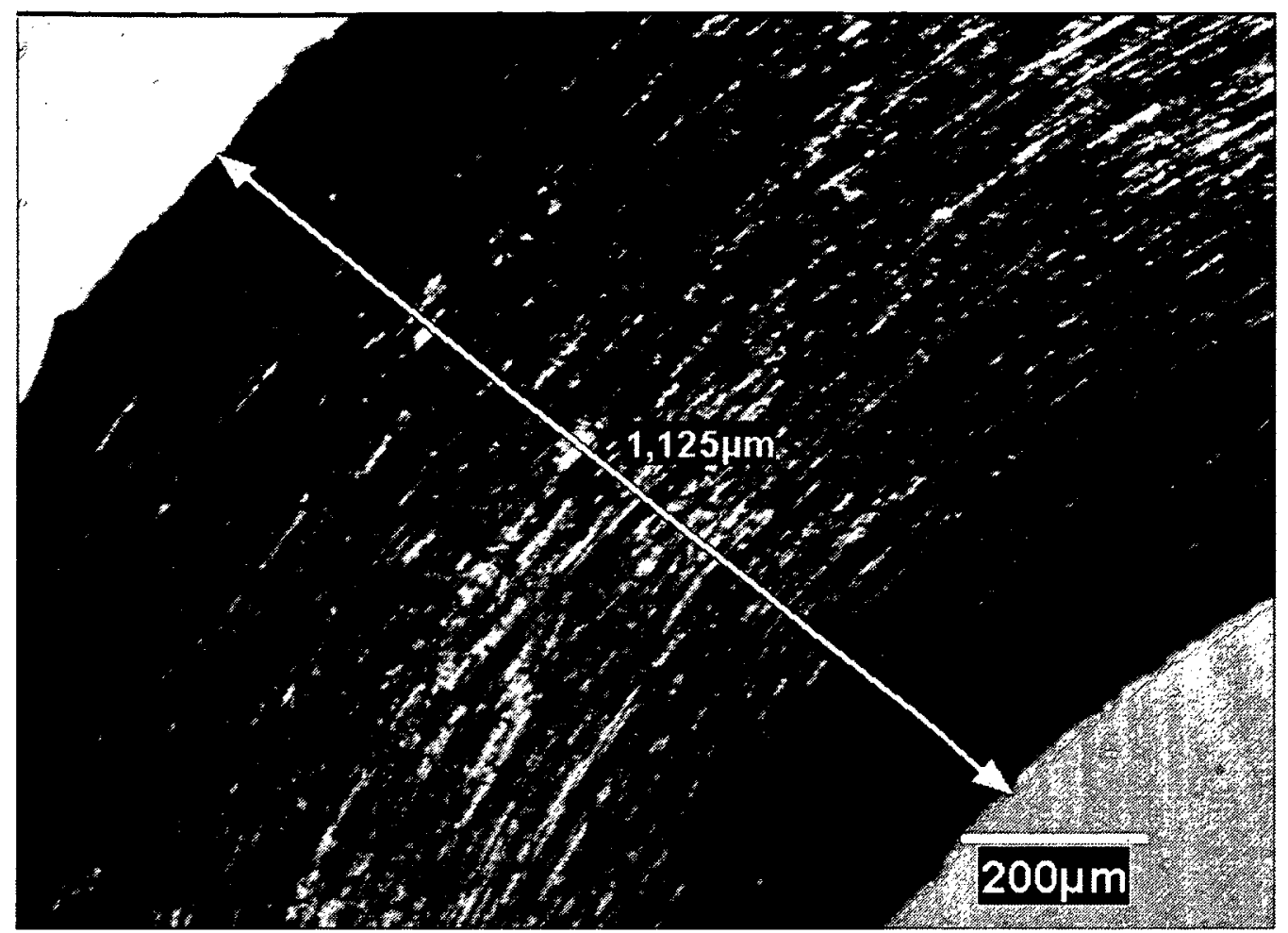

(c)

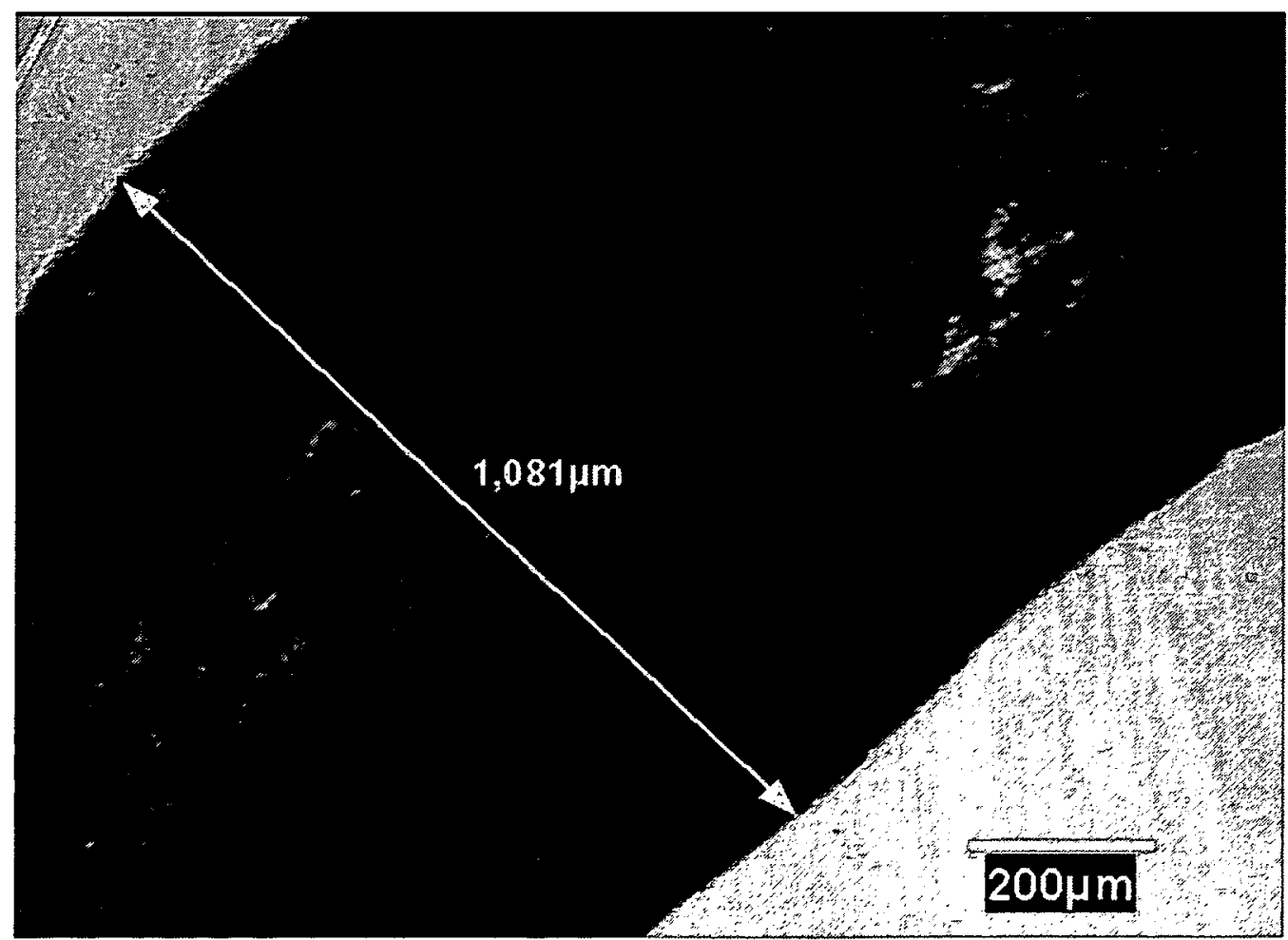

(d) 
(J)

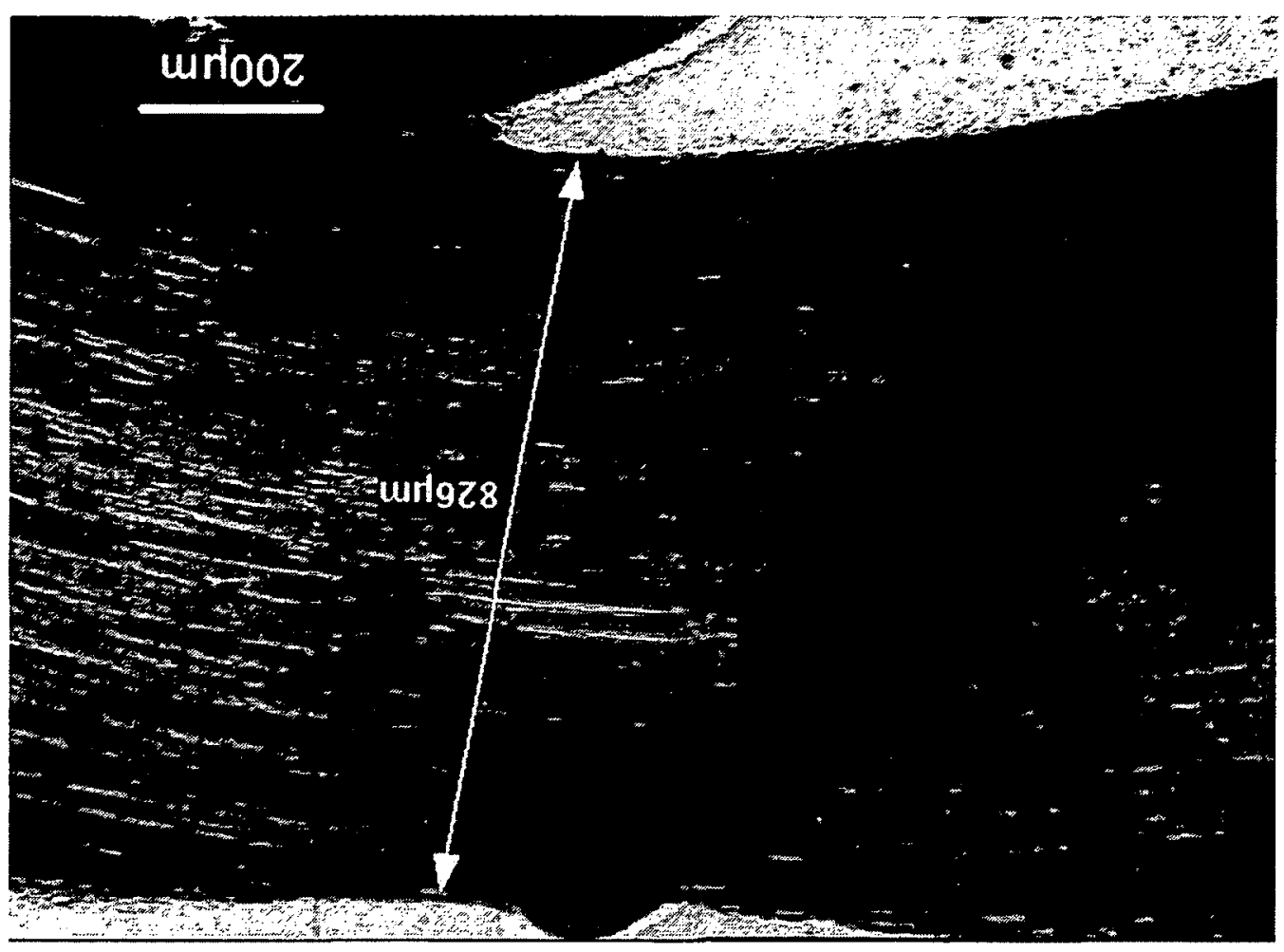

(a)

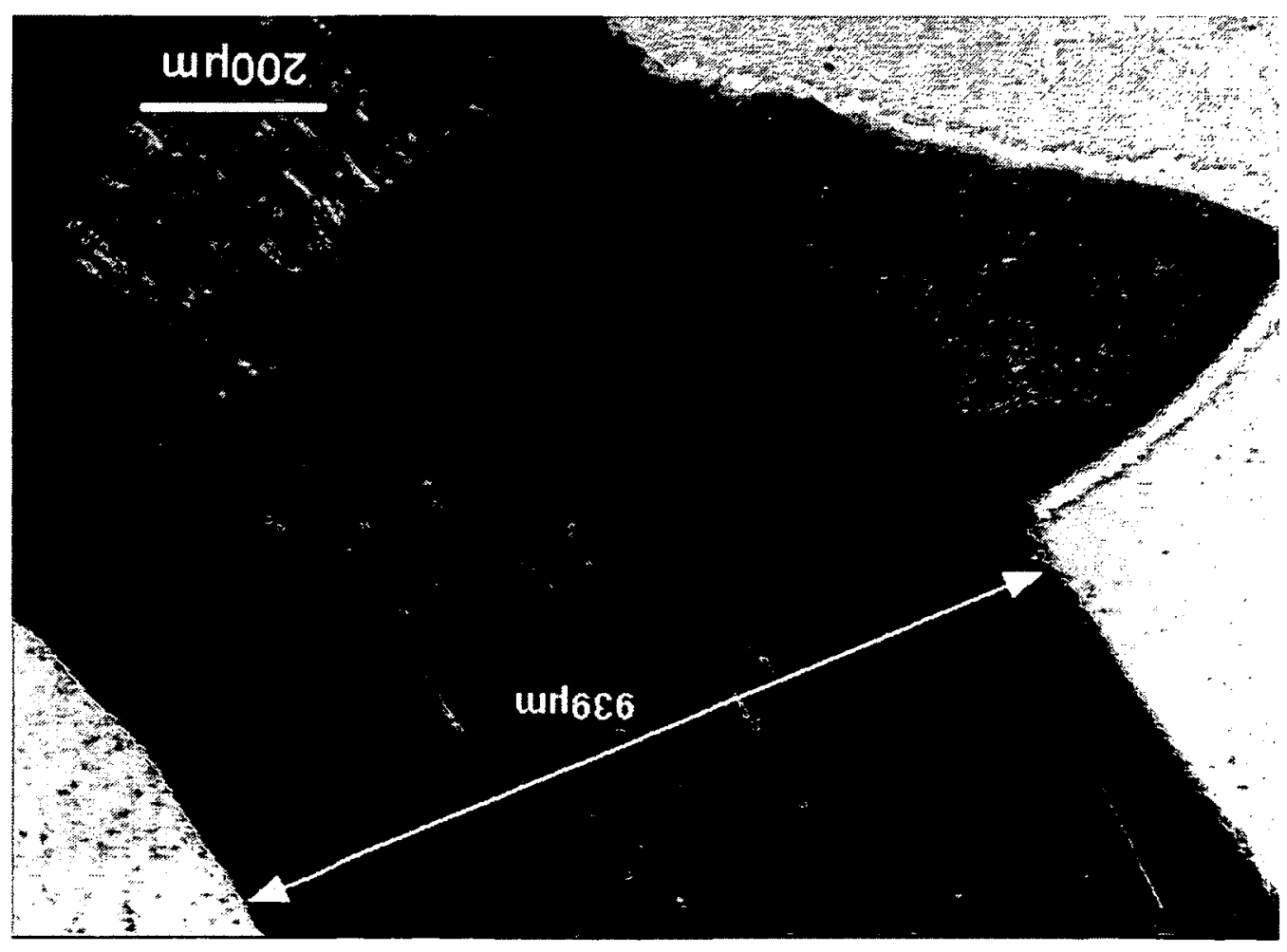




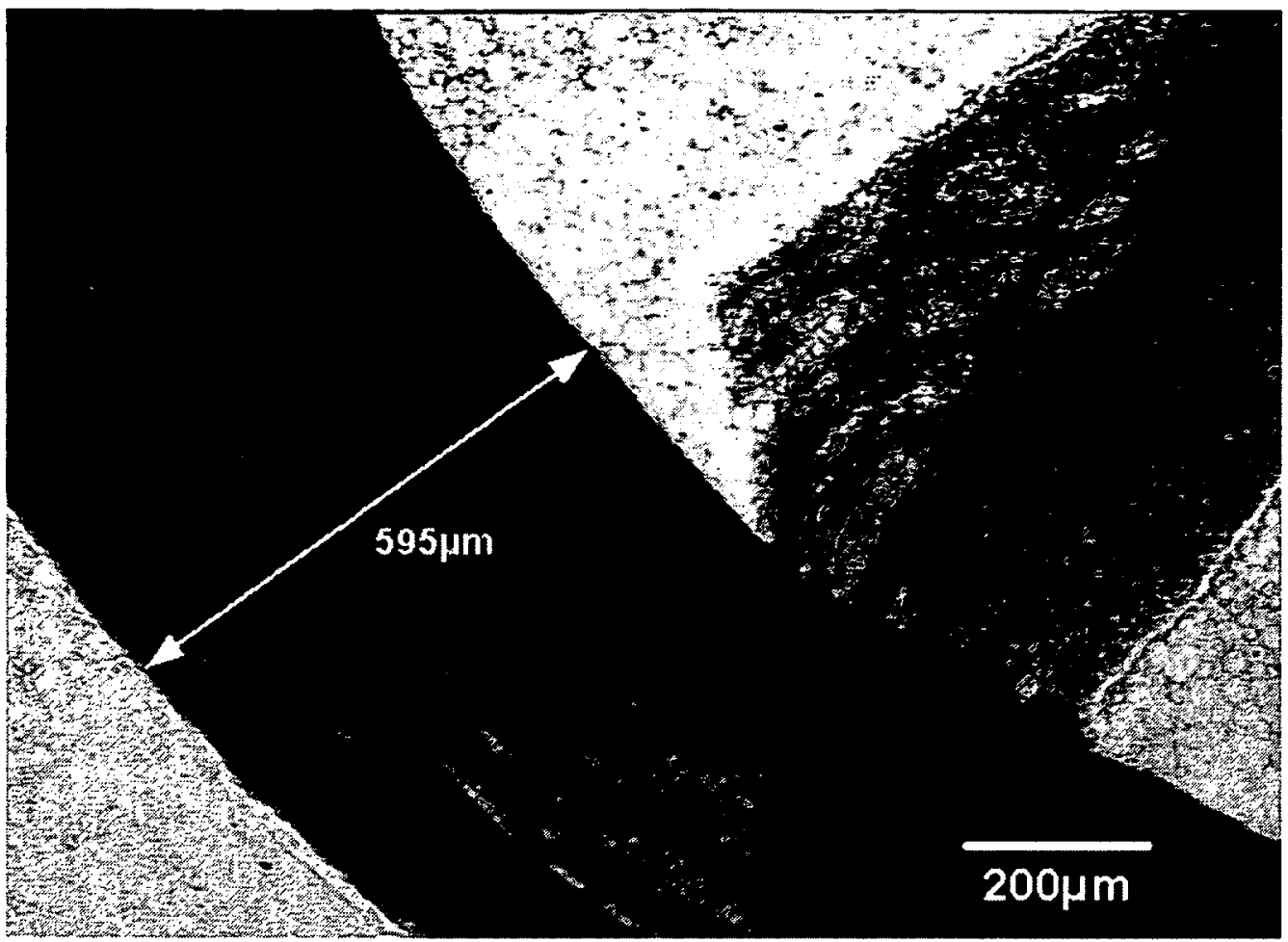

(g)

Fig. 5.25. Worn surfaces: (a) specimen $A$, (b) specimen $K$, (c) specimen $L$, (d) specimen $M$, (e) specimen $N$, (f) specimen $O$ and $(g)$ specimen $P$.

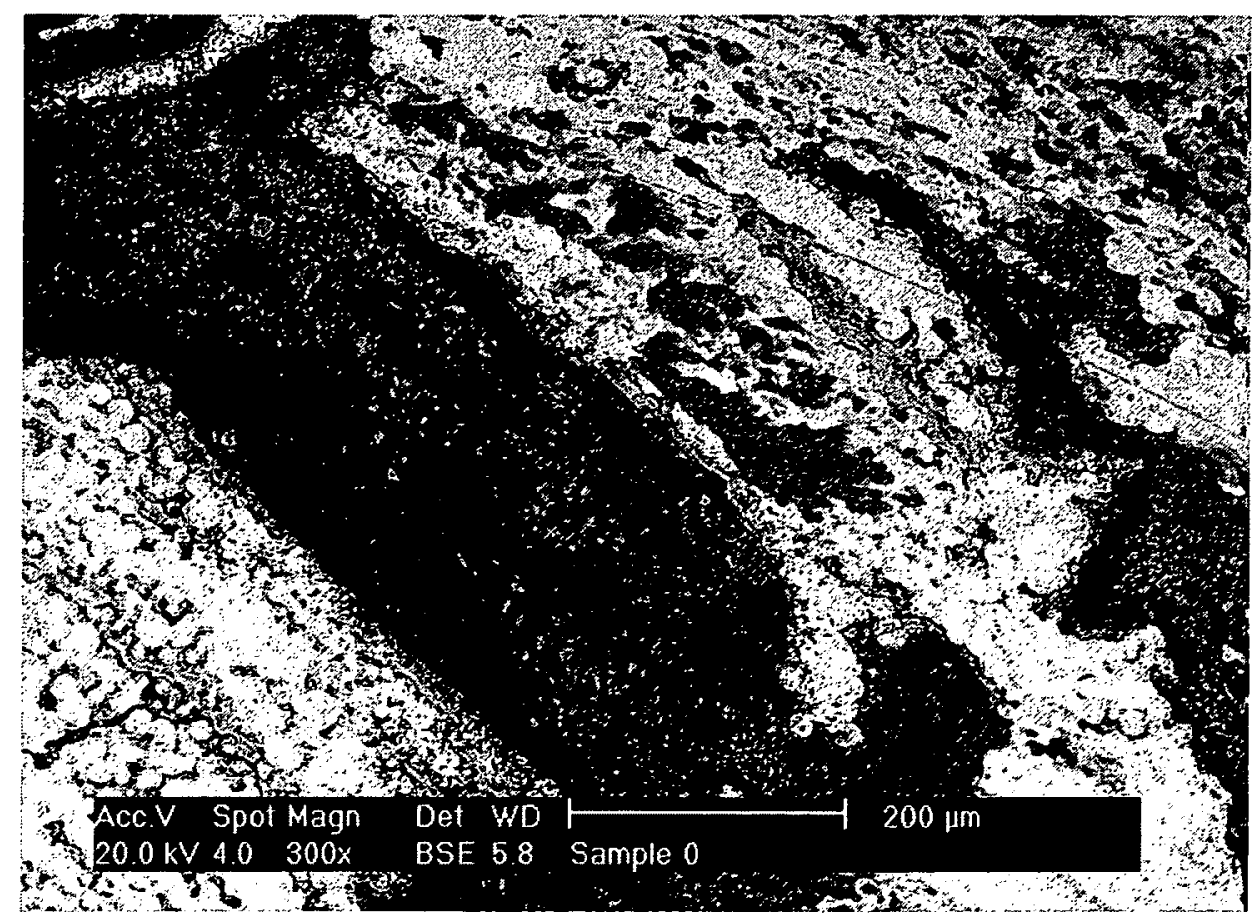

(a) 


\section{Sample 0, wear track}

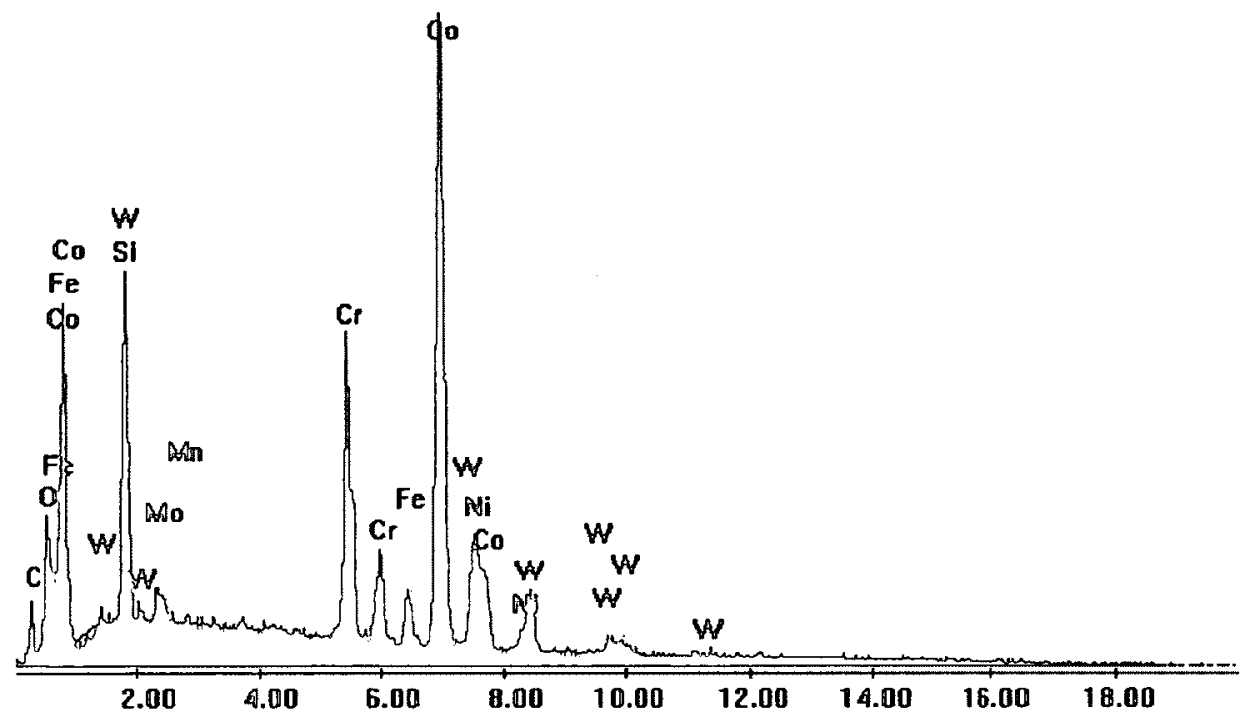

(b)

Fig. 5.26. Worn surface analyses of specimen O: (a) SEM micrograph and (b) EDX spectrum.

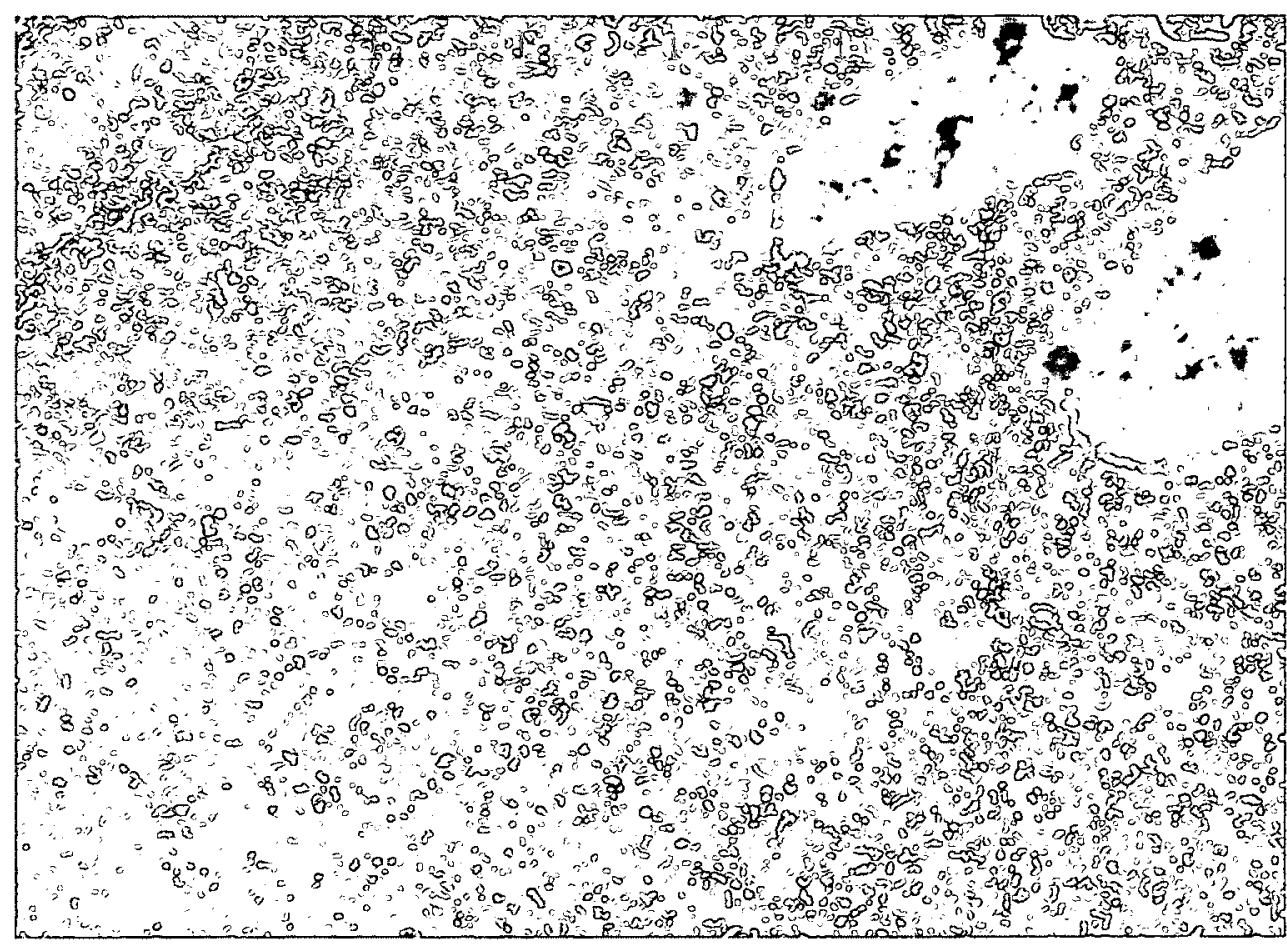

(a) 


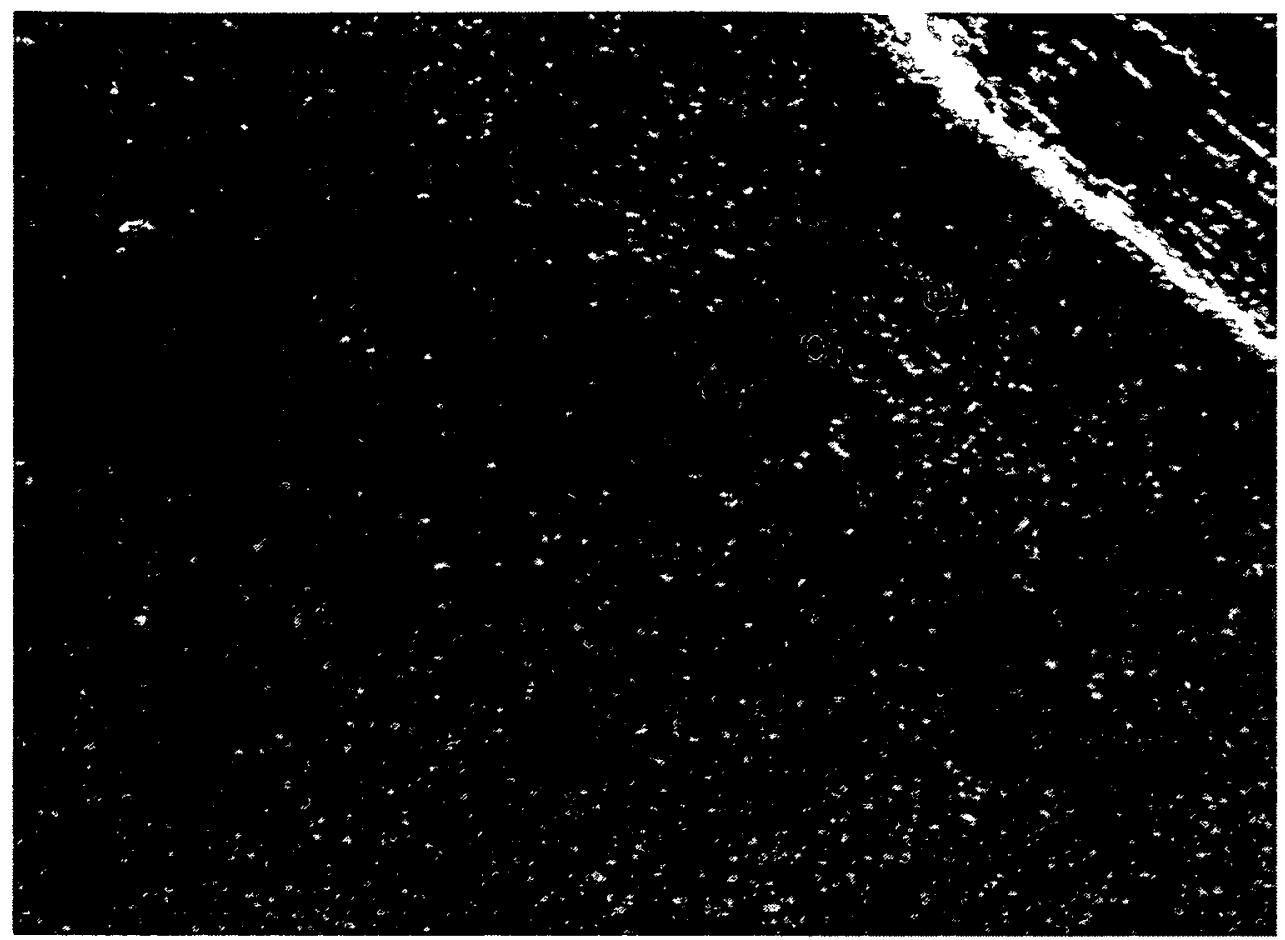

(b)

Fig. 5.27. Microhardness test: (a) specimen F (100x) and (b) specimen $O(100 \times)$.

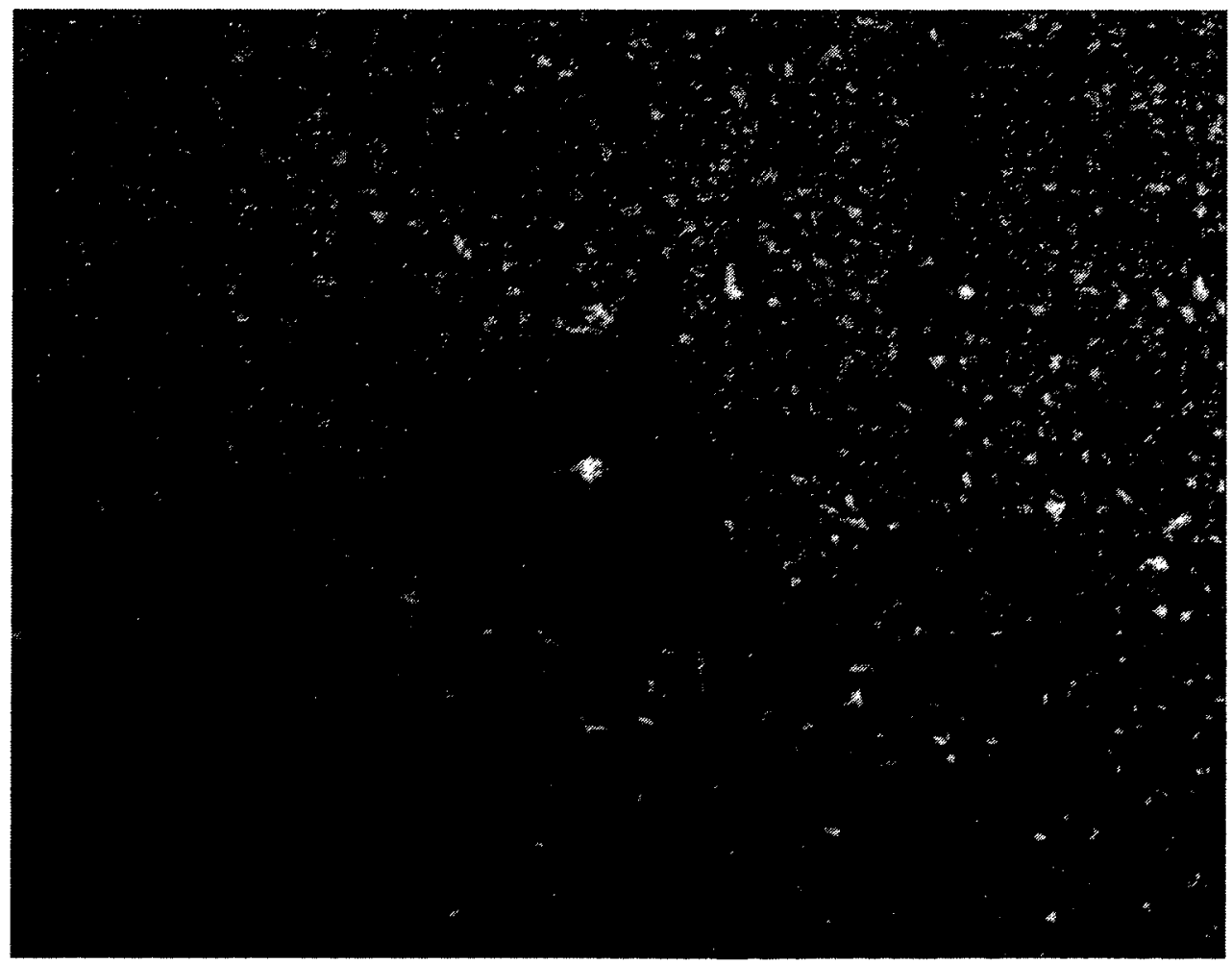

Fig. 5.28. Microhardness test of the carbidic phase in specimen F (500×) 


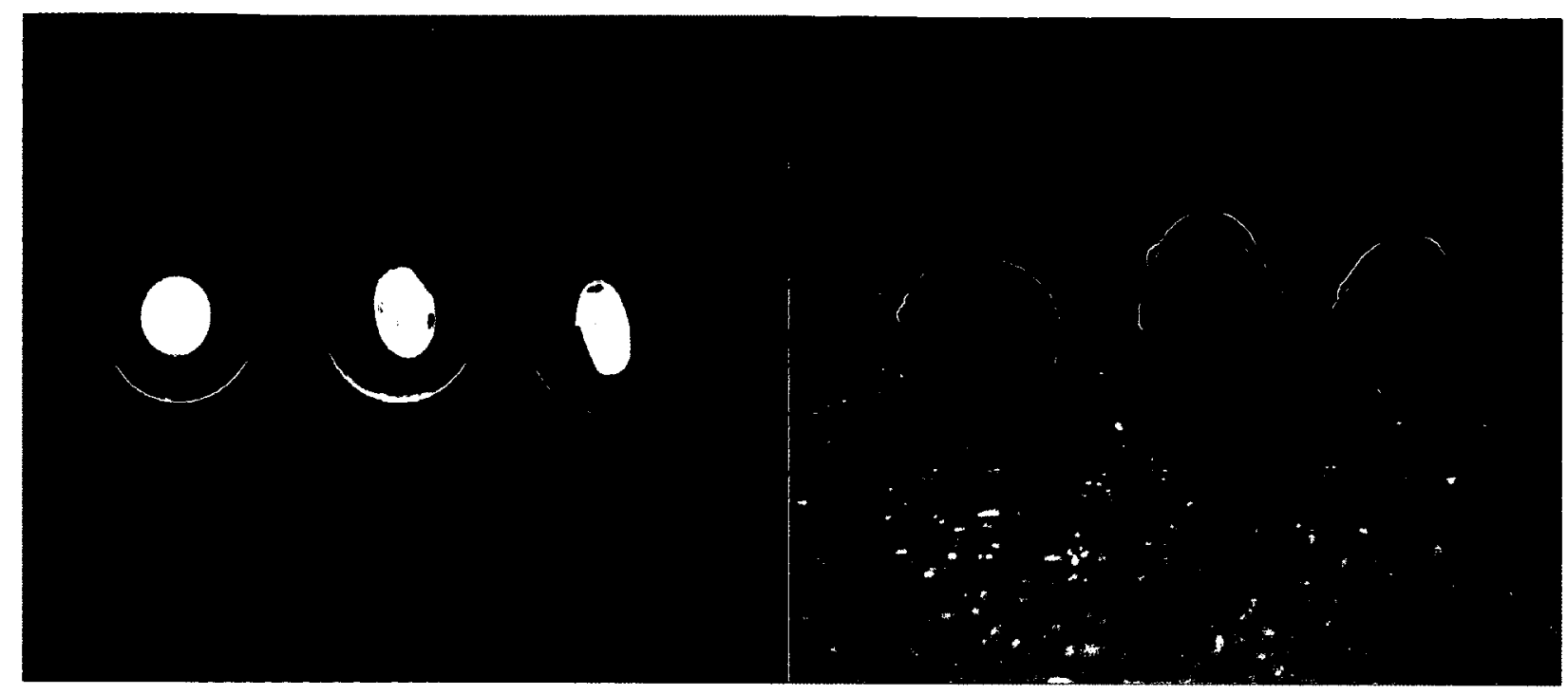

(a)

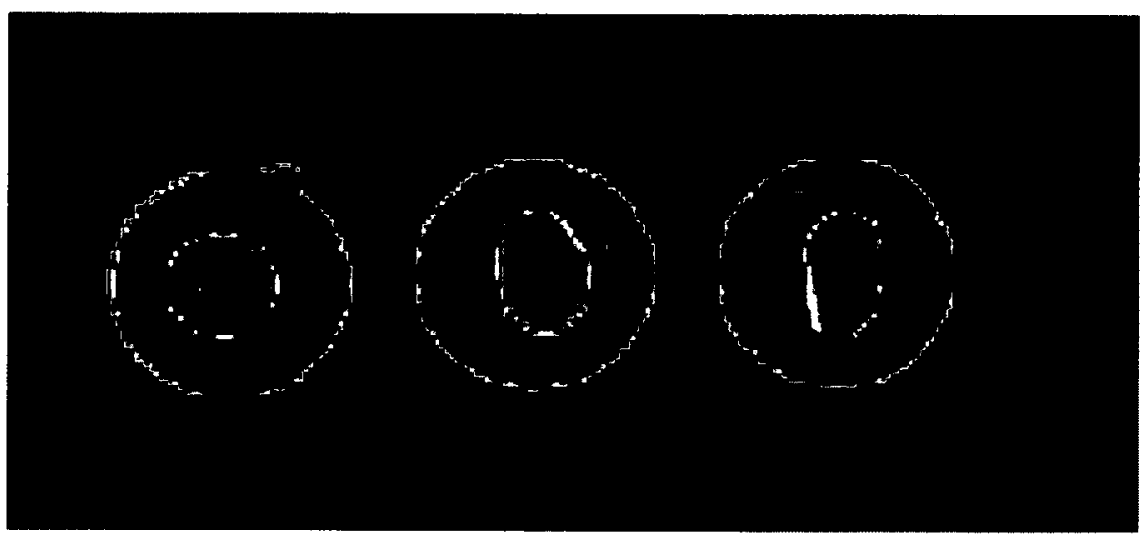

(b)

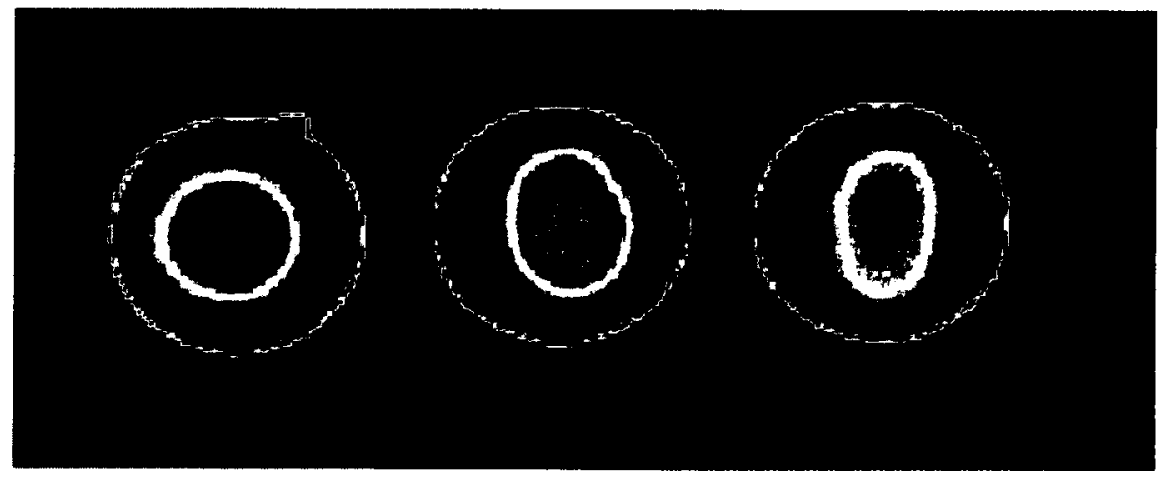

(c)

Fig. 5.29. Thermal conductivity study: (a) tested specimens, (b) PT thermogram at $1 \mathrm{~s}$ and (c) PT thermogram at $8.15 \mathrm{~s}$. 


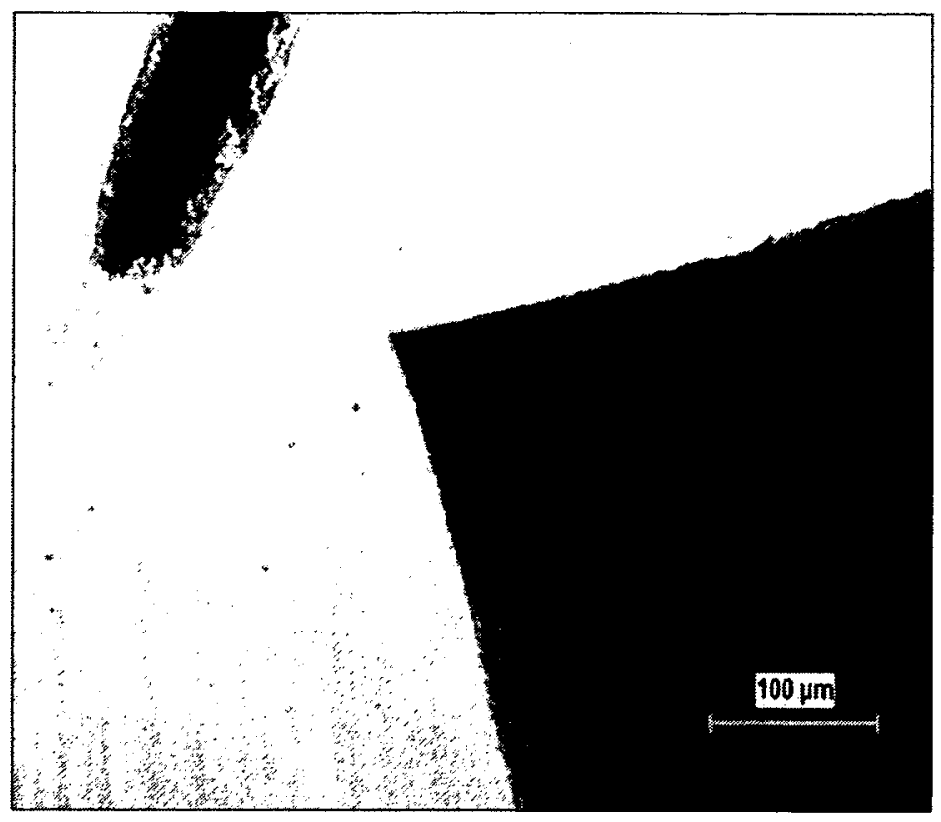

(a)

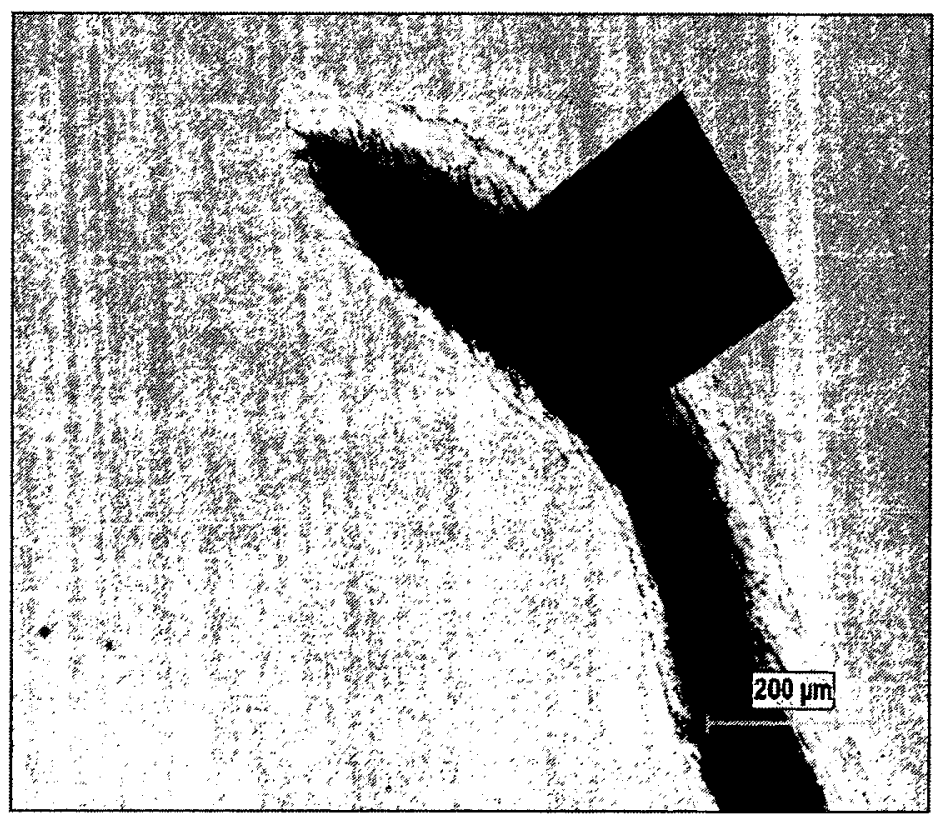

(b)

Fig. 6.1. Vickers hardness indentations on specimen $F$ under load of $60 \mathrm{~kg}$ : (a) close to the interface and (b) at the interface. 


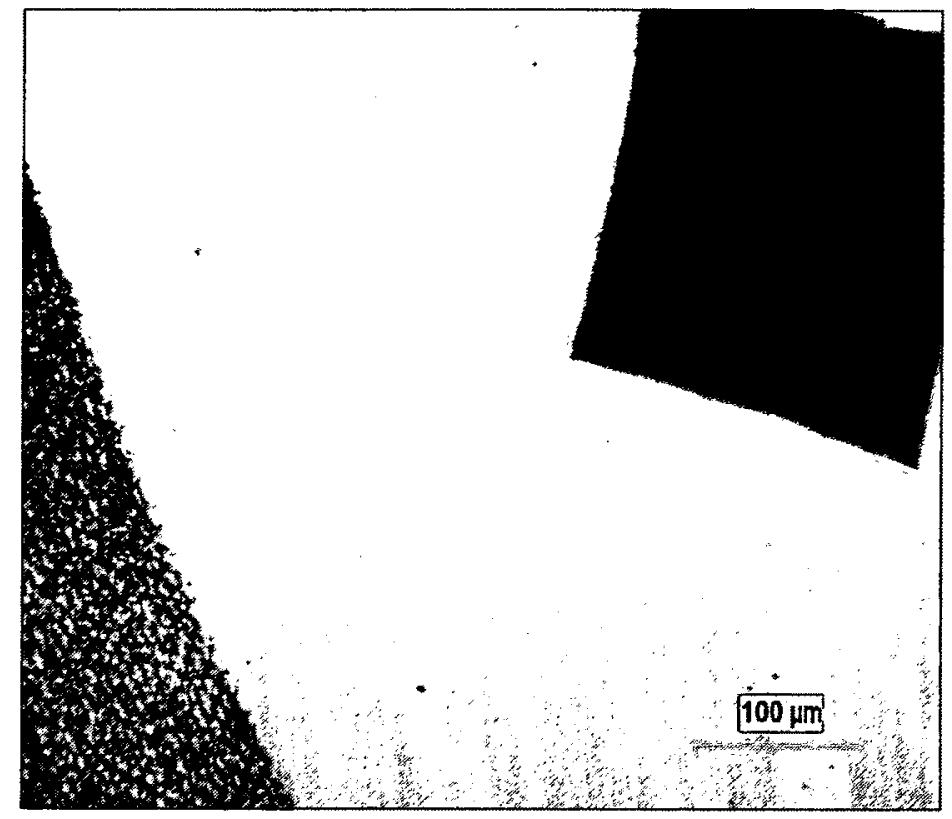

(a)

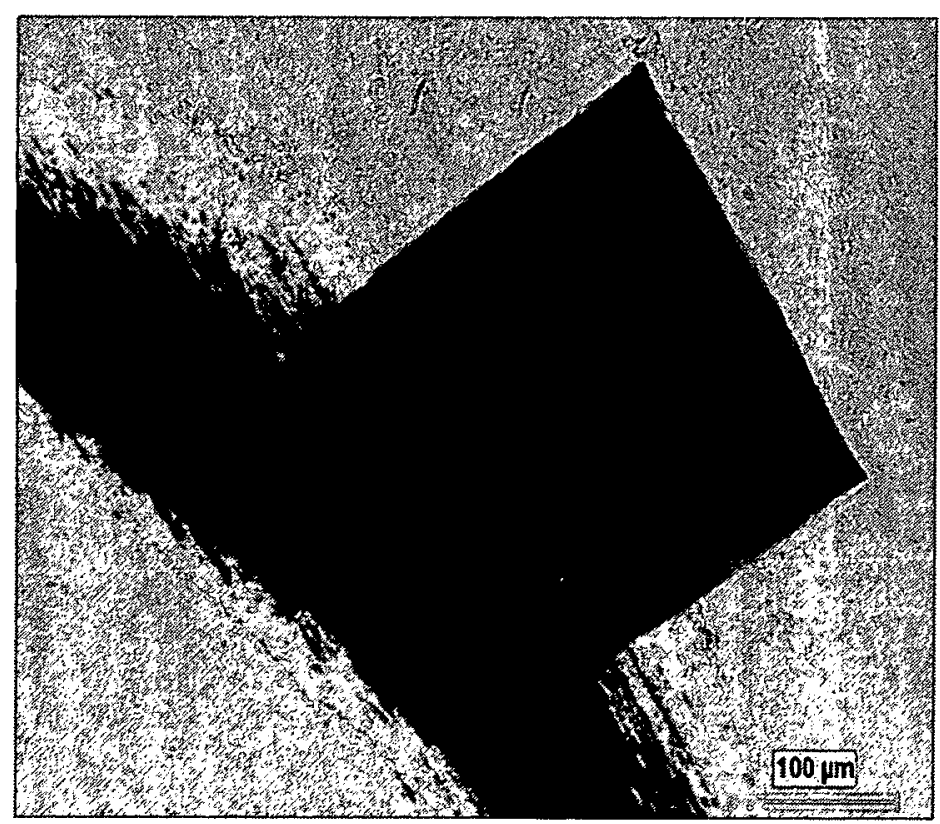

(b)

Fig. 6.2. Vickers hardness indentations on specimen $O$ under load of $60 \mathrm{~kg}$ : (a) close to the interface and (b) at the interface. 


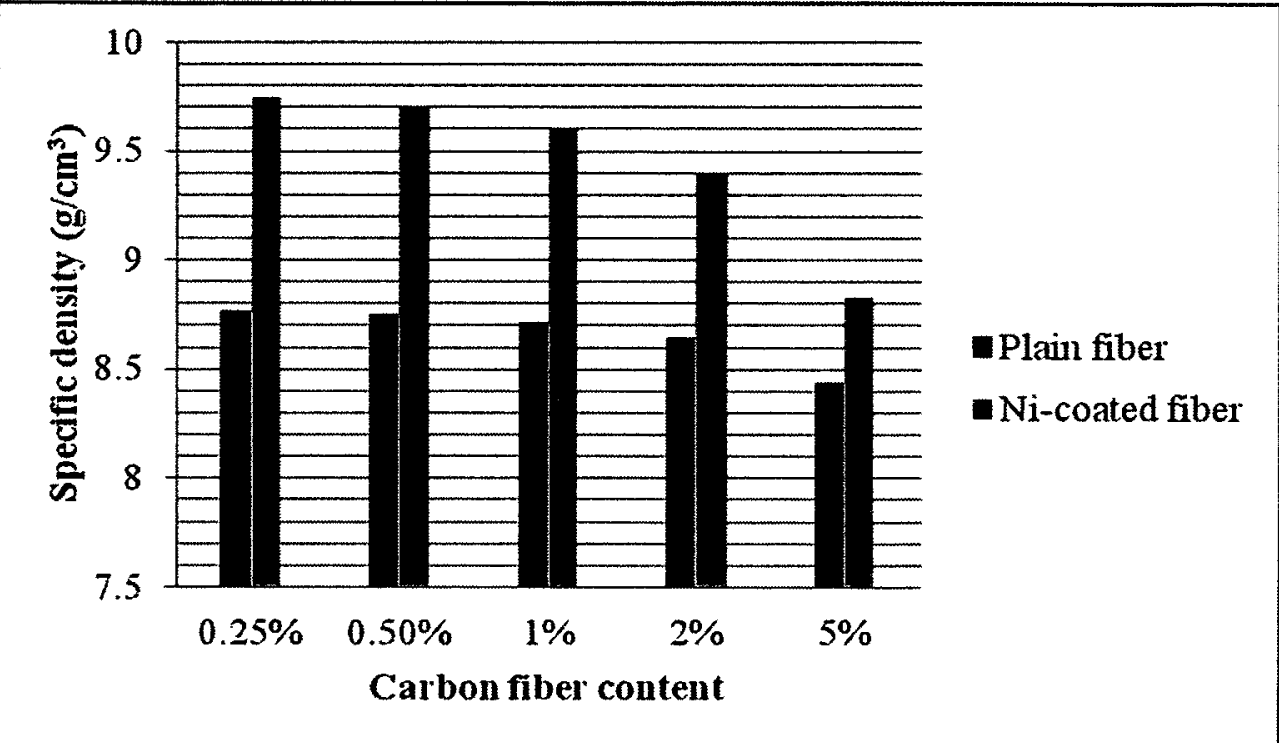

Fig. 6.3. Comparison of specific density between plain fibre reinforced composites and Ni-coated fibre reinforced composites.

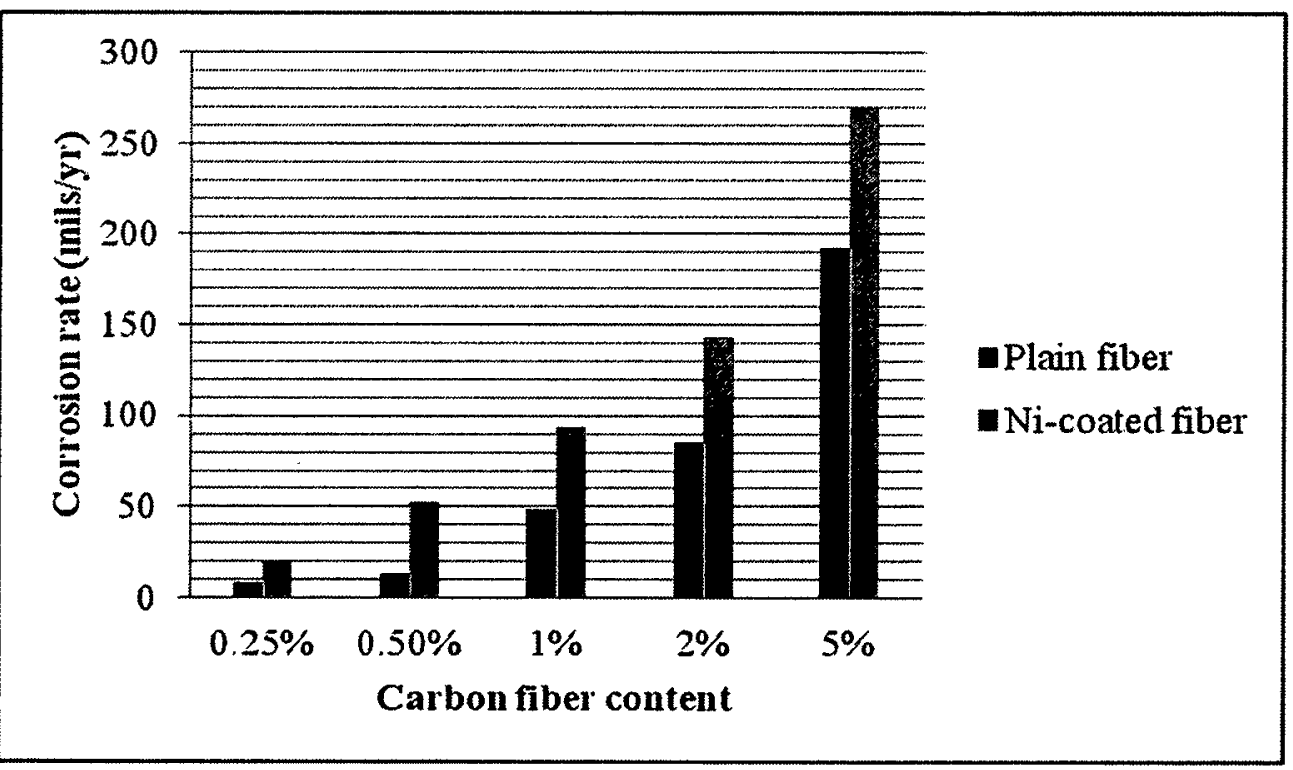

Fig. 6.4. Comparison of corrosion rate between plain fibre reinforced composites and Ni-coated fibre reinforced composites. 


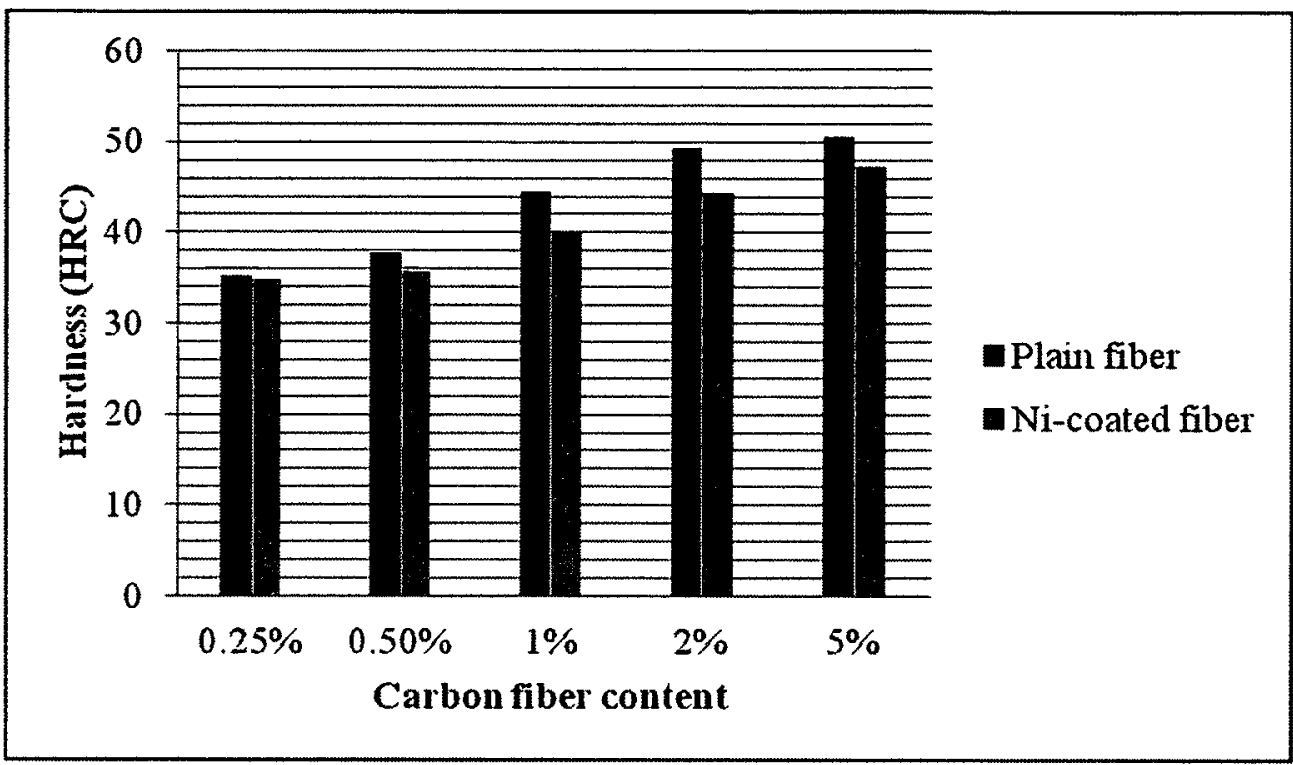

Fig. 6.5. Comparison of hardness between plain fibre reinforced composites and Ni-coated fibre reinforced composites.

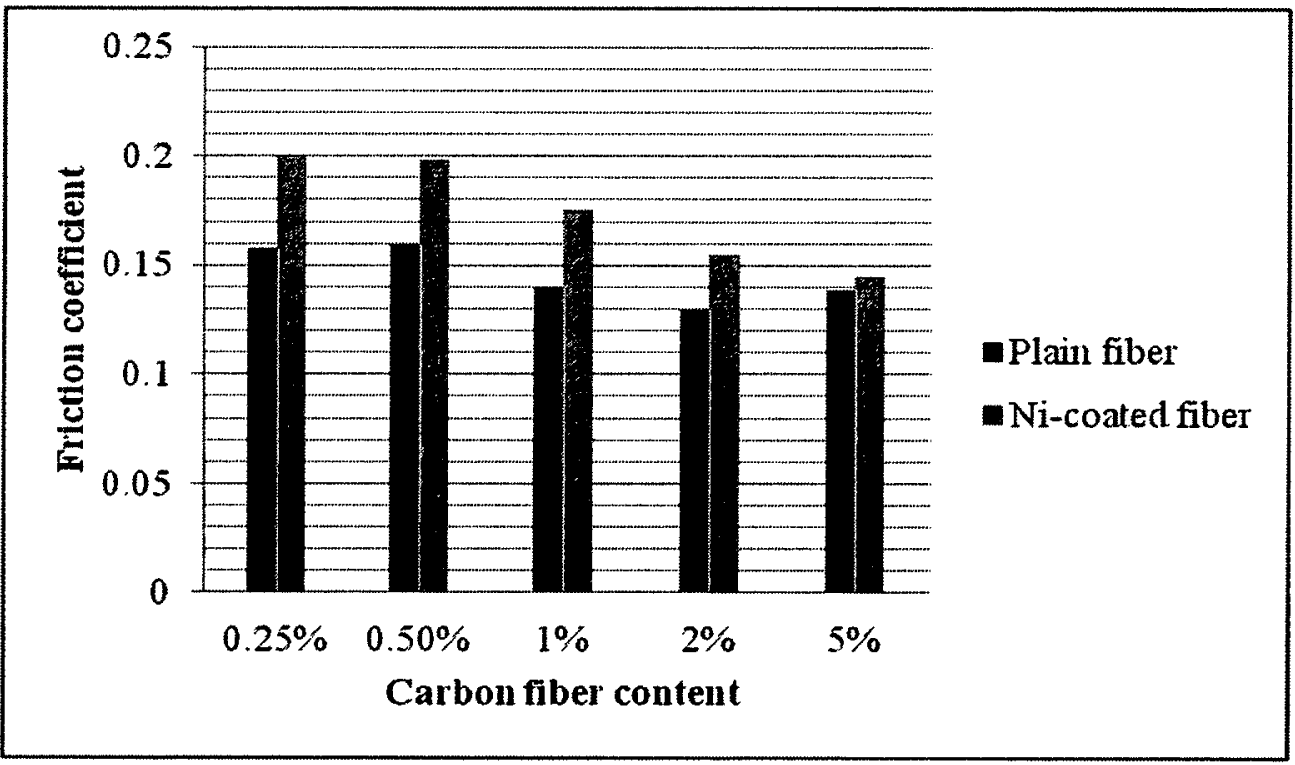

Fig. 6.6. Comparison of friction coefficient between plain fibre reinforced composites and $\mathrm{Ni}$-coated fibre reinforced composites. 


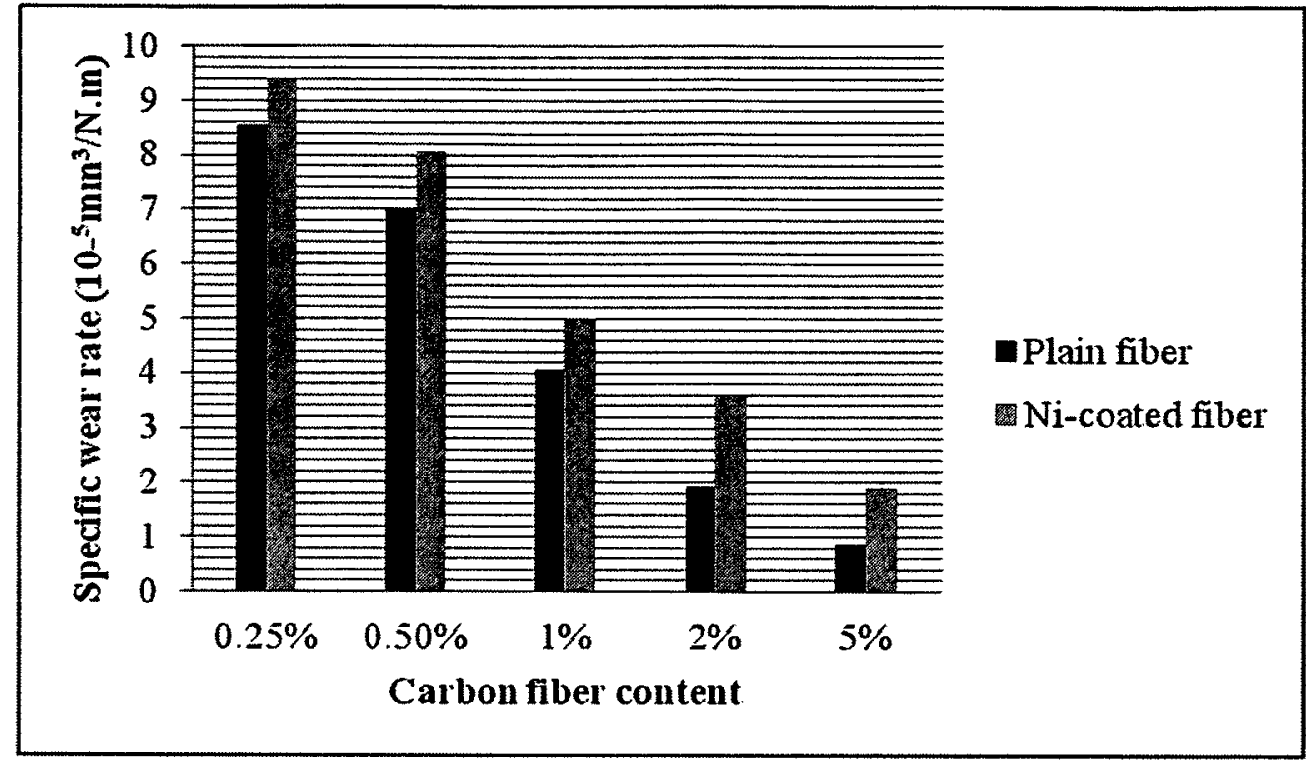

Fig. 6.7. Comparison of wear rate between plain fibre reinforced composites and $\mathrm{Ni}$-coated fibre reinforced composites. 